\title{
Cochrane
}

Library

Cochrane Database of Systematic Reviews

\section{Single or combined immune checkpoint inhibitors compared to first-line platinum-based chemotherapy with or without bevacizumab for people with advanced non-small cell lung cancer} (Review)

Ferrara R, Imbimbo M, Malouf R, Paget-Bailly S, Calais F, Marchal C, Westeel V

Ferrara R, Imbimbo M, Malouf R, Paget-Bailly S, Calais F, Marchal C, Westeel V.

Single or combined immune checkpoint inhibitors compared to first-line platinum-based chemotherapy with or without bevacizumab for people with advanced non-small cell lung cancer.

Cochrane Database of Systematic Reviews 2020, Issue 12. Art. No.: CD013257.

DOI: 10.1002/14651858.CD013257.pub2.

www.cochranelibrary.com

Single or combined immune checkpoint inhibitors compared to first-line platinum-based chemotherapy with or without bevacizumab for people with advanced non-small cell lung cancer (Review) 
TABLE OF CONTENTS

HEADER 1

ABSTRACT

PLAIN LANGUAGE SUMMARY

SUMMARY OF FINDINGS

BACKGROUND

OBJECTIVES

METHODS

RESULTS

Figure 1.

Figure 2.

Figure 3.

Figure 4.

Figure 5.

Figure 6.

Figure 7.

Figure 8.

Figure 9.

DISCUSSION

AUTHORS' CONCLUSIONS

ACKNOWLEDGEMENTS

REFERENCES

CHARACTERISTICS OF STUDIES

DATA AND ANALYSES

Analysis 1.1. Comparison 1: Single immunecheckpoint inhibitors vs chemotherapy, Outcome 1: Overall survival by PD-L1 expression

Analysis 1.2. Comparison 1: Single immunecheckpoint inhibitors vs chemotherapy, Outcome 2: Overall Survival by Tumor Mutational Burden

Analysis 1.3. Comparison 1: Single immunecheckpoint inhibitors vs chemotherapy, Outcome 3: Overall survival by age (PDL1 $\geq$ $1 \%)$

Analysis 1.4. Comparison 1: Single immunecheckpoint inhibitors vs chemotherapy, Outcome 4: Overall survival by age (PDL1 $\geq 50 \%$ )

Analysis 1.5. Comparison 1: Single immunecheckpoint inhibitors vs chemotherapy, Outcome 5: Overall survival by sex (PDL1 $\geq$ $1 \%)$

Analysis 1.6. Comparison 1: Single immunecheckpoint inhibitors vs chemotherapy, Outcome 6: Overall survival by sex (PDL1 $\geq 50 \%$ )

Analysis 1.7. Comparison 1: Single immunecheckpoint inhibitors vs chemotherapy, Outcome 7: Overall survival by smoking status (PDL1 $\geq 1 \%$ )

Analysis 1.8. Comparison 1: Single immunecheckpoint inhibitors vs chemotherapy, Outcome 8: Overall survival by smoking status (PDL1 $\geq 50 \%$ )

Analysis 1.9. Comparison 1: Single immunecheckpoint inhibitors vs chemotherapy, Outcome 9: Overall survival by ECOG PS (PDL1 1 1\%)

Analysis 1.10. Comparison 1: Single immunecheckpoint inhibitors vs chemotherapy, Outcome 10: Overall survival by ECOG PS (PDL1 $\geq 50 \%$ )

Analysis 1.11. Comparison 1: Single immunecheckpoint inhibitors vs chemotherapy, Outcome 11: Overall survival by histological type (PDL1 $1 \%$ )

Analysis 1.12. Comparison 1: Single immunecheckpoint inhibitors vs chemotherapy, Outcome 12: Overall survival by histological type (PDL1 $250 \%$ )

Analysis 1.13. Comparison 1: Single immunecheckpoint inhibitors vs chemotherapy, Outcome 13: Progression free survival by PD-L1 expression

Analysis 1.14. Comparison 1: Single immunecheckpoint inhibitors vs chemotherapy, Outcome 14: Progression Free Survival by Tumor Mutational Burden

Analysis 1.15. Comparison 1: Single immunecheckpoint inhibitors vs chemotherapy, Outcome 15: Overall response rate by PDL1 expression

Single or combined immune checkpoint inhibitors compared to first-line platinum-based chemotherapy with or without bevacizumab for people with advanced non-small cell lung cancer (Review)

Copyright @ 2020 The Cochrane Collaboration. Published by John Wiley \& Sons, Ltd. 
Analysis 1.16. Comparison 1: Single immunecheckpoint inhibitors vs chemotherapy, Outcome 16: Overall Response Rate by Tumor Mutational Burden

Analysis 1.17. Comparison 1: Single immunecheckpoint inhibitors vs chemotherapy, Outcome 17: Adverse Events grade 3-5 .. Analysis 1.18. Comparison 1: Single immunecheckpoint inhibitors vs chemotherapy, Outcome 18: QOL-C30 GHS/QOL - change from baseline to week 15

Analysis 1.19. Comparison 1: Single immunecheckpoint inhibitors vs chemotherapy, Outcome 19: Time to deterioration - QLQLC13

Analysis 1.20. Comparison 1: Single immunecheckpoint inhibitors vs chemotherapy, Outcome 20: HRQoL-QLQC30-change from baseline at week 15-stable-PD-L1 $\geq 50 \%$

Analysis 1.21. Comparison 1: Single immunecheckpoint inhibitors vs chemotherapy, Outcome 21: HRQoL-QLQC30-change from baseline at week 15 -deterioration - PDL1 $\geq 50 \%$

Analysis 1.22. Comparison 1: Single immunecheckpoint inhibitors vs chemotherapy, Outcome 22: HRQoL-QLQC30-change from baseline at week 15 -improvement - PD-L1 $250 \%$

Analysis 2.1. Comparison 2: Combined immune checkpoint inhibitors vs chemotherapy, Outcome 1: Overall Survival by PD-L1 expression

Analysis 2.2. Comparison 2: Combined immune checkpoint inhibitors vs chemotherapy, Outcome 2: Overall Survival by Tumor Mutational Burden

Analysis 2.3. Comparison 2: Combined immune checkpoint inhibitors vs chemotherapy, Outcome 3: Progression Free Survival by Tumor Mutational Burden

Analysis 2.4. Comparison 2: Combined immune checkpoint inhibitors vs chemotherapy, Outcome 4: Overall Response Rate by Tumor Mutational Burden

Analysis 2.5. Comparison 2: Combined immune checkpoint inhibitors vs chemotherapy, Outcome 5: Adverse Events grade 3-5

APPENDICES

HISTORY

CONTRIBUTIONS OF AUTHORS

DECLARATIONS OF INTEREST

SOURCES OF SUPPORT

DIFFERENCES BETWEEN PROTOCOL AND REVIEW 
[Intervention Review]

\title{
Single or combined immune checkpoint inhibitors compared to first-line platinum-based chemotherapy with or without bevacizumab for people with advanced non-small cell lung cancer
}

\author{
Roberto Ferrara1 ${ }^{1}$, Martina Imbimbo², Reem Malouf ${ }^{3}$, Sophie Paget-Bailly4,5, François Calais 6 , Corynne Marchal 7 , Virginie Westeel4,8,9
}

${ }^{1}$ Thoracic Oncology Unit, Fondazione IRCSS Istituto Nazionale dei Tumori, Milano, Italy. ${ }^{2}$ Department of Oncology, Lausanne University Hospital, and Ludwig Institute for Cancer Research, University of Lausanne, Lausanne, Switzerland. ${ }^{3}$ National Perinatal Epidemiology Unit (NPEU), University of Oxford, Oxford, UK. ${ }^{4}$ Methodological and Quality of Life in Oncology Unit, University Hospital of Besançon, Besançon, France. 5Université de Franche-Comté, INSERM, EFS BFC, UMR 1098, Interactions Hôte-Greffon-Tumeur/Ingénierie Cellulaire et Génique, Besançon, France, Besançon, France. ${ }^{6}$ Bibliothèque universitaire de Santé, Université de Franche-Comté, Besançon, France. 7 Université de Franche-Comté, Besançon, France. 8Department of Thoracic Oncology, University Hospital of Besançon, Besançon, France. 9Université Bourgogne Franche-Comté, INSERM, EFS BFC, UMR 1098, Interactions Hôte-Greffon-Tumeur/Ingénierie Cellulaire et Génique, Besançon, France

Contact address: Martina Imbimbo, m.imbimbo1@gmail.com.

Editorial group: Cochrane Lung Cancer Group.

Publication status and date: New, published in Issue 12, 2020.

Citation: Ferrara R, Imbimbo M, Malouf R, Paget-Bailly S, Calais F, Marchal C, Westeel V. Single or combined immune checkpoint inhibitors compared to first-line platinum-based chemotherapy with or without bevacizumab for people with advanced non-small cell lung cancer. Cochrane Database of Systematic Reviews 2020, Issue 12. Art. No.: CD013257. DOI: 10.1002/14651858.CD013257.pub2.

Copyright @ 2020 The Cochrane Collaboration. Published by John Wiley \& Sons, Ltd.

\section{A B S T R A C T}

\section{Background}

Immune checkpoint inhibitors (ICls) targeting the PD-1/PD-L1 axis have changed the first-line treatment of people with advanced nonsmall cell lung cancer (NSCLC). Single-agent pembrolizumab (a PD-1 inhibitor) is currently the standard of care as monotherapy in patients with PD-L1 expression $\geq 50 \%$, either alone or in combination with chemotherapy when PD-L1 expression is less than $50 \%$. Atezolizumab (PD-L1 inhibitor) has also been approved in combination with chemotherapy and bevacizumab (an anti-angiogenic antibody) in first-line NSCLC regardless of PD-L1 expression. The combination of first-line PD-1/PD-L1 inhibitors with anti-CTLA-4 antibodies has also been shown to improve survival compared to platinum-based chemotherapy in advanced NSCLC, particularly in people with high tumour mutational burden (TMB). The association of ipilimumab (an anti CTLA4) and nivolumab (PD-1 inhibitor) has been approved by the US Food and Drug Administration (FDA) in all patients with PD-L1 expression $\geq 1 \%$. Although these antibodies are currently used in clinical practice, some questions remain unanswered, such as the best-treatment strategy, the role of different biomarkers for treatment selection and the effectiveness of immunotherapy according to specific clinical characteristics.

\section{Objectives}

Primary objective: to determine the effectiveness and safety of first-line immune checkpoint inhibitors (ICls), as monotherapy or in combination, compared to platinum-based chemotherapy, with or without bevacizumab for people with advanced NSCLC, according to the level of PD-L1 expression. Secondary objective: to maintain the currency of evidence using a living systematic review approach.

\section{Search methods}

We performed an electronic search of the main databases (Cochrane Lung Cancer Group Trial Register, Cochrane Central Register of Controlled Trials, MEDLINE, Embase) from inception until 21 October 2020 and conferences meetings from 2015 onwards. 


\section{Selection criteria}

We included randomised controlled trials (RCTs) reporting on the efficacy or safety of first-line ICI treatment for adults with advanced NSCLC who had not previously received any anticancer treatment. We included trials comparing single- or double-ICI treatment to standard firstline therapy (platinum-based chemotherapy $+/$ - bevacizumab). All data come from 'international multicentre studies involving adults, age 18 or over, with histologically-confirmed stage IV NSCLC who had not received any previous systemic anti-cancer treatment for advanced disease.

\section{Data collection and analysis}

Three review authors independently assessed the search results and a fourth review author resolved any disagreements. Primary outcomes were overall survival (OS) and progression-free survival (PFS); secondary outcomes were overall objective response rate (ORR) by RECIST $\vee 1.1$, grade 3 to 5 treatment-related adverse events (AEs) (CTCAE v 5.0) and health-related quality of life (HRQoL). We performed metaanalyses where appropriate using the random-effects model for hazard ratios (HRs) or risk ratios (RRs), with $95 \%$ confidence intervals (95\% $\mathrm{Cls})$, and used the $\mathrm{I}^{2}$ statistic to investigate heterogeneity.

\section{Main results}

\section{Main results}

We identified 15 trials for inclusion, seven completed and eight ongoing trials. We obtained data for 5893 participants from seven trials comparing first-line single- (six trials) or double- (two trials) agent ICl with platinum-based chemotherapy, one trial comparing both firstline single- and double-agent ICsI with platinum-based chemotherapy. All trials were at low risk of selection and detection bias, some were classified at high risk of performance, attrition or other source of bias. The overall certainty of evidence according to GRADE ranged from moderate-to-low because of risk of bias, inconsistency, or imprecision. The majority of the included trials reported their outcomes by PD-L1 expressions, with PD-L1 $\geq 50$ being considered the most clinically useful cut-off level for decision makers. Also, iln order to avoid overlaps between various PDL-1 expressions we prioritised the review outcomes according to PD-L1 $\geq 50$.

\section{Single-agent ICI}

In the PD-L1 expression $\geq 50 \%$ group single-agent ICI probably improved OS compared to platinum-based chemotherapy (hazard ratio (HR) $0.68,95 \%$ confidence interval $(\mathrm{Cl}) 0.60$ to $0.76,6 \mathrm{RCTs}, 2111$ participants, moderate-certainty evidence). In this group, single-agent ICl also may improve PFS (HR: $0.68,95 \% \mathrm{Cl} 0.52$ to $0.88,5 \mathrm{RCTs}, 1886$ participants, low-certainty evidence) and ORR (risk ratio (RR): $1.40,95 \% \mathrm{Cl} 1.12$ to 1.75 , 4 RCTs, 1672 participants, low-certainty evidence). HRQoL data were available for only one study including only people with PDL1 expression $\geq 50 \%$, which suggested that single-agent ICI may improve HRQoL at 15 weeks compared to platinum-based chemotherapy (RR: $1.51,95 \% \mathrm{Cl} 1.08$ to $2.10,1 \mathrm{RCT}, 297$ participants, low-certainty evidence).

In the included studies, treatment-related AEs were not reported according to PD-L1 expression levels. Grade 3-4 AEs may be less frequent with single-agent ICl compared to platinum-based chemotherapy (RR: $0.41,95 \% \mathrm{Cl} 0.33$ to $0.50, \mathrm{I}^{2}=62 \%, 5 \mathrm{RCTs}, 3346$ participants, lowcertainty evidence).

More information about efficacy of single-agent ICI compared to platinum-based chemotherapy according to the level of PD-L1 expression and to TMB status or specific clinical characteristics is available in the full text.

\section{Double-agent ICI}

Double-ICI treatment probably prolonged OS compared to platinum-based chemotherapy in people with PD-L1 expression $\geq 50 \%$ (HR: 0.72 , $95 \% \mathrm{Cl} 0.59$ to 0.892 RCTs, 612 participants, moderate-certainty evidence).

Trials did not report data on HRQoL, PFS and ORR according to PD-L1 groups.

Treatment related AEs were not reported according to PD-L1 expression levels. The frequency of grade 3-4 AEs may not differ between double-ICl treatment and platinum-based chemotherapy (RR: $0.78,95 \% \mathrm{Cl} 0.55$ to $1.09, \mathrm{I}^{2}=81 \%, 2 \mathrm{RCTs}, 1869$ participants, low-certainty evidence).

More information about efficacy of double-agent ICI according to the level of PD-L1 expression and to TMB status is available in the full text.

\section{Authors' conclusions}

\section{Authors' conclusions}

The evidence in this review suggests that single-agent ICI in people with NSCLC and PD-L1 $\geq 50 \%$ probably leads to a higher overall survival rate and may lead to a higher progression-free survival and overall response rate when compared to platinum-based chemotherapy and may also lead to a lower rate of adverse events and higher HRQoL. Combined ICI in people with NSCLC and PD-L1 $\geq 50 \%$ also probably leads to a higher overall survival rate when compared to platinum-based chemotherapy, but its effect on progression-free survival, overall response rate and $\mathrm{HRQ} \mathrm{LL}$ is unknown due to a lack of data. The rate of adverse events may not differ between groups.

\section{PLAIN LANGUAGE SUMMARY}

\section{Immunotherapy versus chemotherapy for people with advanced non-small cell lung cancer who have not been not previously been treated}

Single or combined immune checkpoint inhibitors compared to first-line platinum-based chemotherapy with or without bevacizumab for 


\section{Review question}

Is immunotherapy more effective and less toxic than chemotherapy for people diagnosed with non-small cell lung cancer (a subtype of lung cancer) who have not previously been treated and who are not suitable for curative treatment?

\section{Background}

Lung cancer is the leading cause of cancer deaths and non-small cell lung cancer represent more than $85 \%$ of all lung cancer cases. Curative surgery and radiotherapy are not treatment options when the disease is at an advanced stage and until recently these people were offered chemotherapy. Since 2016, immunotherapies (antibodies able to stimulate the immune system against cancer cells) have been shown to improve survival for these patients.

Side effects of immunotherapies are mainly inflammation of the tissues caused by the activation of the immune system against different organs, while chemotherapy usually causes a reduction in the white blood cells and red blood cells, hair loss, nausea and vomiting. In this Cochrane Review, we tried to find out how effective and safe immunotherapies (given alone or as combinations) are compared to standard chemotherapy for people with non-small cell lung cancer who are not suitable for possibly curative treatment.

\section{Study characteristics}

We searched the main databases and records of conference meetings up to 21st October 2020. We included seven studies (5893 participants) comparing immunotherapies (antibodies that interact with specific proteins called immune checkpoints) with chemotherapy for people with non-small cell lung cancer not previously treated.

\section{Key results}

We reported the results by PD-L1 levels (a protein produced by the tumour or immune cells and bound by immune checkpoint inhibitors) In people with more than 50\% of tumour/immune cells expressing PD-L1 protein, single immunotherapy might improve survival with fewer side effects. In addition, treatment with combined immunotherapies may improve survival in both people with high expression of PD-L1 protein.The rate of toxic effects may be the same for people treated with combined immunotherapies or chemotherapy.

\section{Certainty of evidence}

Overall, the certainty of the evidence ranged from moderate to low.

\section{Conclusions}

For people with advanced non-small cell lung cancer with a high expression of PD-L1 protein, immunotherapies alone or combinations of immunotherapies prolonged life compared to chemotherapy. The frequency of side effects may be lower with the use of immunotherapies alone compared to chemotherapy. The frequency of side effects may not differ between combinations of immunotherapies and chemotherapy. 


\section{SUMMARY OF FINDINGS}

Summary of findings 1. Single-immune checkpoint inhibitors (ICIs) compared to chemotherapy for people with advanced non-small cell lung cancer (NSCLC)

Single immune checkpoint inhibitors compared to chemotherapy for people with advanced non-small cell lung cancer

Patient or population: People with advanced non-small cell lung cancer

Setting: Hospital

Intervention: Single immune checkpoint inhibitors

Comparison: First-line, platinum-based chemotherapy

\begin{tabular}{|c|c|c|c|c|c|c|c|}
\hline \multicolumn{2}{|l|}{ Outcomes } & \multicolumn{2}{|c|}{ Anticipated absolute effects ${ }^{\star}(95 \% \mathrm{Cl})$} & \multirow{2}{*}{$\begin{array}{l}\text { Relative ef- } \\
\text { fect } \\
(95 \% \mathrm{CI})\end{array}$} & \multirow{2}{*}{$\begin{array}{l}\text { No of Partici- } \\
\text { pants } \\
\text { (studies) }\end{array}$} & \multirow{2}{*}{$\begin{array}{l}\text { Quality of the } \\
\text { evidence } \\
\text { (GRADE) }\end{array}$} & \multirow[t]{2}{*}{ Comments } \\
\hline & & $\begin{array}{l}\text { Risk with } \\
\text { chemothera- } \\
\text { py }\end{array}$ & $\begin{array}{l}\text { Risk with Single immune } \\
\text { checkpoint inhibitors }\end{array}$ & & & & \\
\hline $\begin{array}{l}\text { Overall sur- } \\
\text { vival (OS) }\end{array}$ & $\begin{array}{l}\text { by PD-L1 ex- } \\
\text { pression } \geq \\
50 \%\end{array}$ & 470 per 1000 & $\begin{array}{l}130 \text { more per } 1000 \\
\text { (90 more to } 170 \text { more) }\end{array}$ & $\begin{array}{l}\text { HR } 0.68 \\
\text { (0.60 to } 0.76)\end{array}$ & $\begin{array}{l}2111 \\
\text { (6 studies) }\end{array}$ & $\begin{array}{l}\text { Moderate }^{1} \\
\oplus \oplus \oplus \ominus\end{array}$ & $\begin{array}{l}\text { Data from Carbone } 2017 \text { and Rizvi } \\
2020 \text { used to estimate the antici- } \\
\text { pated effect at } 12 \text { month. }\end{array}$ \\
\hline $\begin{array}{l}\text { Progres- } \\
\text { sion-free sur- } \\
\text { vival (PFS) }\end{array}$ & $\begin{array}{l}\text { PD-L1 expres- } \\
\text { sion } \geq 50 \%\end{array}$ & 50 per 1000 & $\begin{array}{l}\mathbf{8 0} \text { more per } 1000 \\
\text { (20 more to } 150 \text { more) }\end{array}$ & $\begin{array}{l}\text { HR 0.68, } \\
(0.51 \text { to } 0.88)\end{array}$ & $\begin{array}{l}1886 \\
\text { (5 studies) }\end{array}$ & $\begin{array}{l}\text { Low }^{\mathbf{1 , 2}} \\
\oplus \oplus \ominus \ominus\end{array}$ & $\begin{array}{l}\text { Data from Mok 2019; Reck 2016; } \\
\text { Herbst } 2020 \text { used to estimate the } \\
\text { anticipated effect at } 12 \text { months }\end{array}$ \\
\hline $\begin{array}{l}\text { Overall re- } \\
\text { sponse rate } \\
\text { (ORR) }\end{array}$ & $\begin{array}{l}\text { PDL1 ex- } \\
\text { pression - } \\
\text { PDL1 } \geq 50 \%\end{array}$ & 287 per 1000 & $\begin{array}{l}\mathbf{1 1 5} \text { more per } \mathbf{1 , 0 0 0} \\
\text { (34 more to } 215 \text { more) }\end{array}$ & $\begin{array}{l}\text { RR 1.40, } \\
(1.12 \text { to } 1.75)\end{array}$ & $\begin{array}{l}1672 \\
\text { (4 studies) }\end{array}$ & $\begin{array}{l}\text { Low } 1, \mathbf{2} \\
\oplus \oplus \ominus \ominus\end{array}$ & \\
\hline \multicolumn{2}{|c|}{ Adverse Events grade 3-4 } & 414 per 1,000 & $\begin{array}{l}\mathbf{2 4 4} \text { fewer per } \mathbf{1 , 0 0 0} \\
\text { (217 fewer to } 207 \text { fewer) }\end{array}$ & $\begin{array}{l}\text { RR } 0.41 \\
(0.33 \text { to } 0.50)\end{array}$ & $\begin{array}{l}3346 \\
\text { ( } 5 \text { studies) }\end{array}$ & $\begin{array}{l}\text { Low } \mathbf{1 , 2} \\
\oplus \oplus \ominus \ominus\end{array}$ & $\begin{array}{l}\text { Data presented as overall pooled } \\
\text { result, as data were not available } \\
\text { for this outcome by PD-L1 expres- } \\
\text { sion or by TMB }\end{array}$ \\
\hline $\begin{array}{l}\text { QOL-C30 } \\
\text { GHS/QOL } \\
\text { (range 0-100) }\end{array}$ & $\begin{array}{l}\text { by PDL1 ex- } \\
\text { pression - } \\
\text { PDL1 } \geq 50 \%\end{array}$ & 265 per 1,000 & $\begin{array}{l}\mathbf{1 3 5} \text { more per } \mathbf{1 , 0 0 0} \\
\text { (21 more to } 146 \text { more) }\end{array}$ & $\begin{array}{l}\text { RR } 1.51 \\
\text { (1.08 to } 2.10)\end{array}$ & $\begin{array}{l}297 \\
\text { (1 study) }\end{array}$ & $\begin{array}{l}\text { Low } \mathbf{1 , 3} \\
\oplus \oplus \ominus \ominus\end{array}$ & $\begin{array}{l}\text { A high score indicates a good quali- } \\
\text { ty of life. }\end{array}$ \\
\hline
\end{tabular}

- change

from base- 
*The basis for the assumed risk (e.g. the median control group risk across studies) is provided in footnotes. The corresponding risk (and its $95 \%$ confidence interval) is based on the assumed risk in the comparison group and the relative effect of the intervention (and its $95 \% \mathrm{Cl}$ ).

Cl: Confidence interval; HR: Hazard ratio; RR: Risk ratio

\section{GRADE Working Group grades of evidence}

High certainty: we are very confident that the true effect lies close to that of the estimate of the effect.

Moderate certainty: we are moderately confident in the effect estimate; the true effect is likely to be close to the estimate of the effect, but there is a possibility that it is substantially different.

Low certainty: our confidence in the effect estimate is limited; the true effect may be substantially different from the estimate of the effect.

Very low certainty: we have very little confidence in the effect estimate; the true effect is likely to be substantially different from the estimate of effect.

1Downgraded one point due to risk of other bias (Carbone 2017 differences in baseline characteristics; Mok 2019 several protocol amendments), performance bias (Carbone 2017, Hellmann 2018, Reck 2016, Rizvi 2020, Sezer 2020), or of attrition bias (Hellmann 2018 and Rizvi 2020).

2Downgraded one point due to inconsistency.

${ }^{3}$ Downgraded one point due to imprecision. Results come from one single trial with relatively small sample size, or the confidence interval includes both clinically relevant values and clinically irrelevant values, thus limiting confidence to draw conclusions on an apparent lack of effect or a possible relevant effect.

\section{Summary of findings 2. Combined immune checkpoint inhibitors (ICIs) compared to chemotherapy for people with advanced non-small cell lung} cancer (NSCLC)

\section{Combined immune checkpoint inhibitors compared to chemotherapy for people with advanced non-small cell lung cancer}

Patient or population: people with advanced non-small cell lung cancer

Setting: Hospital

Intervention: Combined immune checkpoint inhibitors

Comparison: First-line, platinum-based chemotherapy

\begin{tabular}{|c|c|c|c|c|c|c|c|}
\hline \multirow[t]{2}{*}{ Outcomes } & & \multicolumn{2}{|c|}{ Anticipated absolute effects ${ }^{\star}(95 \% \mathrm{Cl})$} & \multirow{2}{*}{$\begin{array}{l}\text { Relative ef- } \\
\text { fect } \\
(95 \% \mathrm{CI})\end{array}$} & \multirow{2}{*}{$\begin{array}{l}\text { № of partici- } \\
\text { pants } \\
\text { (studies) }\end{array}$} & \multirow{2}{*}{$\begin{array}{l}\text { Certainty of } \\
\text { the evidence } \\
\text { (GRADE) }\end{array}$} & \multirow[t]{2}{*}{ Comments } \\
\hline & & $\begin{array}{l}\text { Risk with } \\
\text { Chemothera- } \\
\text { py }\end{array}$ & $\begin{array}{l}\text { Risk with combined im- } \\
\text { mune checkpoint in- } \\
\text { hibitors }\end{array}$ & & & & \\
\hline $\begin{array}{l}\text { Overall Sur- } \\
\text { vival (OS) }\end{array}$ & $\begin{array}{l}\text { by PD-L1 expres- } \\
\text { sion - PD-L1 } \geq 50 \%\end{array}$ & 510 per 1,000 & $\begin{array}{l}\mathbf{1 1 0} \text { more per } \mathbf{1 , 0 0 0} \\
\text { (40 more to } 162 \text { more) }\end{array}$ & $\begin{array}{l}\text { HR } 0.72 \\
(0.59 \text { to } 0.89)\end{array}$ & $\begin{array}{l}612 \\
(2 \mathrm{RCTs})\end{array}$ & $\begin{array}{l}\text { Moderate }^{\mathbf{1}} \\
\oplus \oplus \oplus \ominus\end{array}$ & $\begin{array}{l}\text { Rizvi } 2020 \text { data used to calcu- } \\
\text { late anticipated absolute ef- } \\
\text { fects }\end{array}$ \\
\hline
\end{tabular}




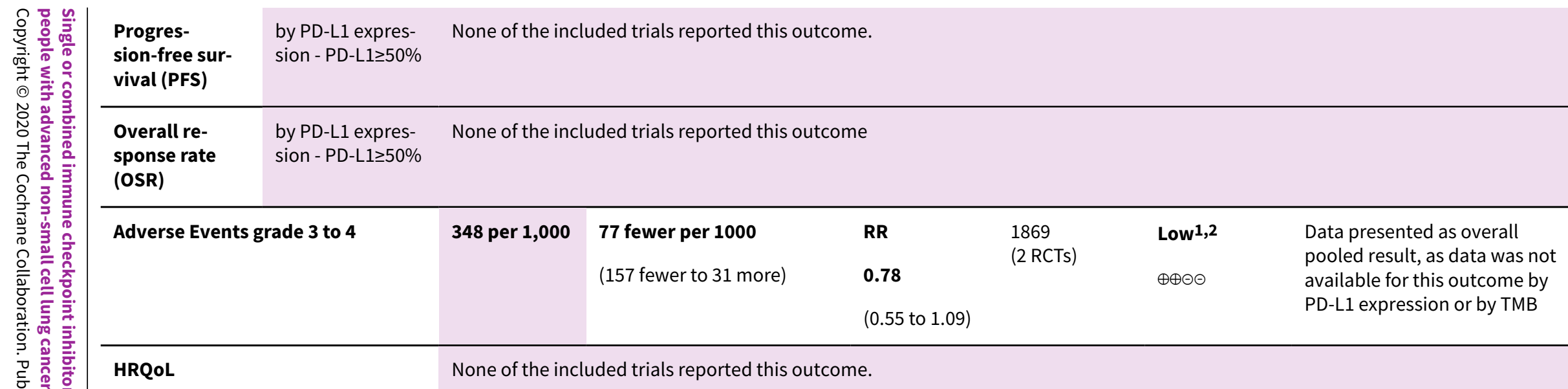

${ }^{*}$ The risk in the intervention group (and its 95\% confidence interval) is based on the assumed risk in the comparison group and the relative effect of the intervention (and its $95 \% \mathrm{Cl})$.

Cl: Confidence interval; HR: Hazard ratio; RR: Risk ratio;

\section{GRADE Working Group grades of evidence}

High certainty: we are very confident that the true effect lies close to that of the estimate of the effect.

Moderate certainty: we are moderately confident in the effect estimate; the true effect is likely to be close to the estimate of the effect, but there is a possibility that it is substantially different.

Low certainty: our confidence in the effect estimate is limited; the true effect may be substantially different from the estimate of the effect.

Very low certainty: we have very little confidence in the effect estimate; the true effect is likely to be substantially different from the estimate of effect.

1Downgraded one point due to risk of bias in Hellmann 2018 high risk of attrition bias because of TMB analysis or of other bias because of the several protocol amendments affecting the outcomes measured, Rizvi 2020 high risk of attrition bias).

2Downgraded one point due to inconsistency 


\section{B A C K G R O U N D}

\section{Description of the condition}

Lung cancer is one of the leading causes of cancer death worldwide (Bray 2018). Non-small cell lung cancer (NSCLC), which accounts for $85 \%$ to $90 \%$ of lung cancers (Novello 2016), includes two major histological types: non-squamous carcinoma and squamous carcinoma.The expression of PD-L1 (a protein produced by the tumour or immune cells and bound by immune checkpoint inhibitors (ICI)) on NSCLC cells may vary. Approximately $23 \%$ of NSCLCs have $\geq 50 \%$ tumour cells expressing PD-L1 (PD-L1 tumour proportional score (TPS) $\geq 50 \%$ ), $38 \%$ have $1 \%$ to $49 \%$ of tumour cells expressing PD-L1 (PD-L1 TPS $1 \%$ to $49 \%$ ), and $39 \%$ of NSCLCS do not express PD-L1 (PD-L1 TPS <1\%) (Garon 2015).

Until recently, the standard first-line treatments for NSCLC with no driver mutations (epidermal growth factor receptor (EGFR), anaplastic lymphoma kinase (ALK) or receptor tyrosine kinase (ROS1) genomic aberrations) were platinum-based chemotherapy including platinum and a non-pemetrexed third-generation agent for squamous histology, and platinum-pemetrexed or platinumpaclitaxel with or without bevacizumab (an anti-angiogenic agent) for non-squamous histology, achieving median progression-free survival (PFS) of five to six months, and median overall survival (OS) of 11 (squamous histology) to 17 (non-squamous histology) months (Lopez-Chavez 2012; Paz-Ares 2013; Scagliotti 2008).

\section{Description of the intervention}

The advent of $\mathrm{ICl}$, has dramatically changed the choice of firstline treatment. Pembrolizumab, a programmed cell death protein-1 (PD-1) inhibitor, has been shown to prolong survival in people with NSCLC and PD-L1 TPS $\geq 1 \%$ (Garon 2015). In 2016, pembrolizumab, was approved by the Food and Drug Administration (FDA) and the European Medicines Agency (EMA) as monotherapy in treatmentnaive metastatic NSCLC with a PD-L1 TPS $\geq 50 \%$. Moreover, in April 2019, the FDA expanded pembrolizumab indication to first-line treatment of people with stage IV or stage-III NSCLC, who are not candidates for surgical resection or definitive chemoradiation and who have no EGFR or ALK genomic aberrations and PD-L1 TPS $\geq 1 \%$. Similarly, first-line cemiplimab (a PD-1 inhibitor), and atezolizumab (a PD-L1 inhibitor) given as single agents were associated with promising survival and responses compared to platinum-based chemotherapy in NSCLC people with PD-L1 expression on tumour cells or immune cells $\geq 50 \%$ (Sezer 2020) and $\geq 5 \%$ (Peters 2017), respectively.

Nivolumab, another PD-1 inhibitor, has also been reported to lead to durable responses and interesting survival outcomes in first-line setting, as monotherapy (Gettinger 2016); or in combination with ipilimumab, an anti-cytotoxic T-lymphocyte-associated protein 4 (CTLA-4) agent (Ready 2019), and this combination has been recently approved by the FDA in people with NSCLC and PD-L1 expression $\geq 1 \%$.

Among PD-1/PD-L1 inhibitors and anti-CTLA-4 agents combinations, tremelimumab, a CTLA4 inhibitor plus durvalumab, a PD-1 inhibitor, showed anti-tumour activity regardless of PD-L1 expression in people with advanced NSCLC (Antonia 2016).

\section{How the intervention might work}

Checkpoint inhibitors are a class of humanised immunoglobulins that target and inhibit molecules responsible for the physiological 'off-switch' of immune cells to prevent an excessive and uncontrolled immune response. Their inhibition activates Tlymphocytes and enhances the adaptive anti-cancer immune response.

Nivolumab,pembrolizumab and cemiplimab (immunoglobulin (Ig) G4 monoclonal antibodies) bind PD-1 on immune cells, blocking their interaction with PD-L1 and PD-L2 expressed by tumour cells (Ishida 1992). Atezolizumab, durvalumab, and avelumab (IgG1 monoclonal antibodies) bind PD-L1 on tumour cells, preventing interaction with PD-1. Both classes of drugs counteract PD-1 mediated inhibition of the immune response.

CTLA-4 is expressed by T cells and, after binding to CD80/CD86, activates an inhibitory downstream signal in human lymphocytes (Hathcock 1993). Ipilimumab (IgG1) and tremelimumab (IgG2) block human CTLA4, inducing T-cell activation, proliferation, and intratumoural infiltration, with improved anti-cancer immune response.

Combining PD-L1 inhibitors and anti-CTLA4 agents might improve antitumour immunity because PD- 1 and CTLA4 modulate effector T-cell activation, proliferation, and function through distinct complementary mechanisms (Okazaki 2013). Double immune checkpoint blockade may have a relevant role in particular for tumours with elevated tumour mutation burden (TMB) (Lawrence 2013), which are known to be highly sensitive to immunotherapy. Furthermore, recent evidence has shown that the primary target of PD-1 inhibition is the downstream pathway of CD28, a COstimulatory receptor that can bind to CD80/CD86 (Hui 2017). Considering that CD80/CD86 is also a ligand for CTLA-4, the combination of CTLA-4 and PD-1 inhibitors may have a synergistic effect with high activation of CD80/CD86 - CD28 axis and an increased antitumour immune response.

\section{Why it is important to do this review}

Recent advances in immunotherapy have led to the approval of immunotherapy alone or in combination with chemotherapy as first-line treatment for NSCLC, according to PD-L1 expression. Double immune checkpoint blockade is also emerging as a treatment option in NSCLC with high TMB. Some questions remain unanswered, such as the best treatment strategy (i.e. immunotherapy as single agent or in combination), the role of different biomarkers (i.e. PD-L1 TPS, TMB) for treatment selection and the effectiveness of immunotherapy according to specific clinical characteristics. In fact, people with advanced NSCLC and uncontrolled brain metastases, auto-immune disorders, steroid dependency, and poor performance status are usually excluded from clinical trials aiming to test treatment with single-agent or combined immune checkpoint inhibitors. Furthermore, people with oncogene-addicted (i.e. EGFR mutated or ALK rearranged) NSCLC have also been excluded from these clinical trials. Although this review did not attempt to address specific questions for these subgroups of patients, particular attention will be paid to potentially interesting clinical and pathological variables that may influence the outcomes of immune checkpoint inhibitors, such as gender, age, smoking status, histology and PD-L1 expression. In fact, subgroup analyses from randomised clinical trials in 
people with pre-treated NSCLC have raised doubts about the immunotherapy benefit for elderly people (Brahmer 2015) or those people who have never smoked (Borghaei 2015), or among those with NSCLC with low/negative PD-L1 expression (Borghaei 2015). Furthermore, a recent meta-analysis showed that in patients with cancer, the magnitude of benefit from ICI may be sex-dependent, with worse outcomes reported for women (Conforti 2018).

\section{Living systematic review approach}

After publication of this review, we are planning to maintain it as a living systematic review (LSR). This means that we will search the literature continually, on a monthly basis and will incorporate any new evidence as it becomes available (Elliott 2017). A living systematic review approach is appropriate for this review because the topic is important for clinical decision-making; current evidence is unlikely to provide certainty; and a very active research programme is ongoing. Indeed, the review authors are aware of several ongoing trials and believe that incorporating results from these trials in a timely manner is crucial, as these findings may have an impact on review conclusions.

\section{O B JECTIVES}

\section{Primary}

To determine the effectiveness and safety of first-line immune checkpoint inhibitors, as monotherapy or in combination compared to platinum-based chemotherapy with or without bevacizumab for people with advanced non-small cell lung cancer (NSCLC), according to the level of PD-L1 expression.

\section{Secondary}

To maintain the currency of evidence using a living systematic review approach.

\section{METHODS}

\section{Criteria for considering studies for this review}

\section{Types of studies}

We included randomised controlled trials (RCTs) reporting on the effectiveness or safety of immune checkpoint inhibitors (ICls) as first-line treatment for people with advanced NSCLC, with or without blinding. We applied no language or publication status restrictions, and when sufficient data was available, we included meeting abstracts and unpublished online data.

\section{Types of participants}

We included studies involving participants with metastatic NSCLC or locally advanced NSCLC not susceptible to curative treatment. People should have not received any first-line systemic treatment. We did not apply any restrictions for age, gender, drug dosage, or treatment duration.

\section{Types of interventions}

We considered studies for inclusion if researchers reported one or more of the following comparisons.

- Single-agent immune checkpoint inhibitors (ICls) versus standard first-line therapy (doublet chemotherapy \pm bevacizumab).
- Doublet immune checkpoint inhibitors (ICls) versus standard first-line therapy (doublet chemotherapy \pm bevacizumab).

A doublet chemotherapy regimen includes any platinum-based doublet along with a third-generation agent (i.e. gemcitabine, vinorelbine, taxanes, pemetrexed).

Although we acknowledge that a lot of evidence is available on the combination of first-line immune checkpoint inhibitors and chemotherapy, this ongoing Cochrane systematic review examines the potential benefit of immunotherapy and chemotherapy combinations compared to first-line chemotherapy or single-agent ICI (Syn 2018).

\section{Types of outcome measures}

\section{Primary outcomes}

- Overall survival (OS): defined as time from randomisation to death from any cause (https://www.cancer.gov/publications/ dictionaries/cancer-terms/def/overall-survival).

- Progression-free survival (PFS): defined as time from randomisation to cancer progression or death from any cause. (https://www.cancer.gov/publications/dictionaries/ cancer-terms/def/progression-free-survival).

\section{Secondary outcomes}

- Overall objective response rate (ORR): measured by Response Evaluation Criteria in Solid Tumours (RECIST) v.1.1 (Eisenhauer 2009); guidelines for response criteria for use in trials testing immunotherapeutics (iRECIST) (Seymour 2017); or immunerelated RECIST (irRECIST) (Nishino 2013).

- Health-related quality of life (HRQoL): measured via validated generic or disease-specific questionnaires, or validated items

- Treatment-related adverse events (AEs): any AEs as reported by the included trials individually. We investigated the incidence of grade 3 (severe or medically significant but not immediately lifethreatening; hospitalisation or prolongation of hospitalisation indicated; disabling; limiting self-care activities of daily living) and grade 4 events (life-threatening consequences; urgent intervention indicated) based on the Common Terminology Criteria for Adverse Events (CTCAE) and Patient-Reported Outcomes CTCAE (PRO-CTCAE) (Kluetz 2016). We also checked the included trials for incidence of grade $5 \mathrm{AEs}$ (deaths related to adverse events).

\section{Search methods for identification of studies}

\section{Electronic searches}

We searched the following electronic databases from inception to 21st October 2020.

- Cochrane Lung Cancer Group Trial Register.

- Cochrane Central Register of Controlled Trials (CENTRAL), in the Cochrane Library.

- MEDLINE, accessed via PubMed.

- Embase.

We did not apply any restrictions on language of publication.

We have presented the search strategies for CENTRAL, MEDLINE, and Embase in Appendix 1, Appendix 2, and Appendix 3, respectively. 
We searched all databases using both controlled vocabulary (namely, medical subject headings (MeSH) in MEDLINE and EMTREE in Embase) and a wide range of free-text terms. We performed the MEDLINE search using the Cochrane highly sensitive search strategy and precision-maximising version (2008 version), as described in the Cochrane Handbook for Systematic Reviews of Interventions (Chapter 6.4.11.1, and detailed in Box 6.4.b) (Higgins 2011b).

We also conducted searches in the following clinical trials registries to identify unpublished and ongoing trials until 22nd January 2020.

- ClinicalTrials.gov.

- WHO International Clinical Trials Registry Platform (ICTRP).

\section{Living systematic review approach}

In approaching this as a living systematic review, we will keep on searching the following databases monthly, using auto-alerts if possible.

- Cochrane Lung Cancer Group Trials Register.

- Cochrane Central Register of Controlled Trials (CENTRAL), in the Cochrane Library.

- MEDLINE, accessed via PubMed.

- Embase.

For other electronic databases and electronic sources (including trials registries), we will set up auto-alerts (if possible) to deliver a monthly search yield by email. If auto-alerts are not possible, we will manually search these electronic databases and electronic sources each month.

We will review search methods and strategies at least yearly, to ensure that they reflect any terminology changes in the topic area or in the databases. For example, we will review search methods and strategies whenever researchers become aware of a new drug meeting the definition for either intervention or comparison and used for the review population.

\section{Searching other resources}

We handsearched the references of eligible studies to identify additional studies for inclusion.

We searched the meeting abstracts of conferences from the following sources from 2015 onwards.

- World Conference on Lung Cancer (WCLC).

- European Society for Medical Oncology (ESMO).

- European Society for Medical Oncology Immuno-Oncology congress (ESMO IO).

- European Lung Cancer Conference (ELCC).

- American Society of Clinical Oncology (ASCO).

- American Association of Cancer Research (AACR).

We retrieved clinical study reports about the checkpoint inhibitors from the European Medicines Agency (EMA) website.

\section{Living systematic review considerations}

In developing this living systematic review, we noted when these key conferences are held and will keep on searching conference proceedings when published.
We will contact corresponding authors of ongoing studies as we identify them and will ask them to advise when study results are available, or to share early or unpublished data. We will contact the corresponding authors of any newly included studies for advice regarding other relevant studies.

We will manually search the reference lists of all newly included studies.

\section{Data collection and analysis}

\section{Selection of studies}

Three review authors (CM, RM and $\mathrm{MI}$ ) screened independently all titles and abstracts retrieved by electronic searches. These review authors obtained the full texts for all relevant studies and checked independently the eligibility of each study against review eligibility criteria. We pursued discordant evaluations by discussion to reach consensus. When necessary to reach consensus, we involved a fourth review author (RF).

We immediately screened all new citations retrieved by our searches and will keep on doing it on a monthly basis.

\section{Data extraction and management}

The review authors developed a data extraction form. Two review authors (RM, RF) independently extracted relevant data. To reach consensus, we involved a third review author when necessary (MI). We were not blinded to the names of study authors nor to the institutions where studies were conducted and funded. When we encountered multiple publications for the same study, we choose the first publication dealing with the primary endpoint in this review as a study identifier (study ID).

We extracted the following details from each included study.

- Source: citation, study name if applicable, and contact details.

- Study details: study design, location, setting (type and stage of disease), sample size, and study start date and completion date, study follow-up

- Characteristics of participants: inclusion and exclusion criteria, number of participating centres, number of participants, participant and tumour characteristics (age, sex, ethnicity, smoking status, performance status, histology, molecular status, tumour-node-metastasis (TNM) stage, PD-L1 expression, tumour mutational burden (TMB).

- Characteristics of interventions (e.g. drugs, doses, cycle duration).

- Outcomes: primary and secondary outcomes with definitions and time points.

- Results: number of participants allocated to each group, and for each outcome of interest, sample size, summary data for each group, estimate of effect with confidence interval and $P$ value and subgroup analyses, and whether analyses have been performed by intention-to-treat (ITT) or per-protocol methods.

- Miscellaneous: funding source.

\section{Assessment of risk of bias in included studies}

Two review authors (RM, RF) independently applied the Cochrane 'Risk of bias' tool per Chapter 8 of the Cochrane Handbook for Systematic Reviews of Interventions, to assess quality and potential biases across studies eligible for inclusion in this review (Higgins 
2011). We rated each domain of the tool as having 'low', 'high', or 'unclear' risk of bias at study level and for each outcome if possible, and we supported the rating of each domain by providing a brief description. We summarised risk of bias for each outcome within a study by considering all domains relevant to the outcome (i.e. both study-level entries, such as allocation sequence concealment, and outcome-specific entries, such as blinding). We provided a figure to summarise the risk of bias, similar to Figure 8.6.C, as presented in the Cochrane Handbook for Systematic Reviews of Interventions ( Higgins 2011).

When two review authors could not reach consensus, we consulted with a third review author (SPB).

Using the Cochrane 'Risk of bias' tool, we considered the following domains.

- Selection bias: random sequence generation.

- Selection bias: allocation concealment.

- Performance bias: blinding of participants and personnel.

- Detection bias: blinding of outcome assessment.

- Attrition bias: incomplete outcome data for outcomes related to efficacy and safety.

- Reporting bias: selective reporting of outcomes.

- Other bias: such as inclusion of patients concordant to pre-specified number of participants needed for calculation, unplanned interim analyses, and unbalanced baseline characteristics across arms.

\section{Measures of treatment effect}

For time-to-event outcomes - OS and PFS - we used hazard ratios (HRs) to measure treatment effects. We reported each HR along with the $95 \%$ confidence Interval $(\mathrm{Cl})$. An HR of one indicates that the hazard rate is equivalent between experimental and control groups, and an HR other than one indicates differences in hazard rates between the two groups. We extracted the HR from the included studies when it was available. When it was not reported in the included study, we tried to calculate the HR by using KaplanMeier survival curves and the dedicated methods of Parmar and Tierney (Parmar 1998; Tierney 2007).

For dichotomous outcomes $\mathrm{AE}$ and $\mathrm{ORR}$, we used risk ratios (RRs) and $95 \% \mathrm{Cls}$ if possible.

For dichotomous outcomes related to OS and PFS at specific time points, we used survival rates and $95 \% \mathrm{Cls}$.

For continuous outcomes (HRQOL), we used mean differences (MDs) between treatment arms when a similar scale was implemented to measure outcomes, and planned to use standardised mean differences (SMDs) if different scales were used to measure the same outcome. We confirmed that higher scores for continuous outcomes have the same meaning for the particular outcome, explained the direction, and reported if directions were reversed.

\section{Unit of analysis issues}

The primary unit of analysis was the participant.

\section{Studies with multiple treatment groups}

For studies with multiple comparison groups that compared two or more intervention groups versus the same control group, we first tried to combine groups to create a single pair-wise comparison. We calculated within-study correlation as recommended in the Cochrane Handbook for Systematic Reviews of Interventions (Higgins 2011).

When studies employed a cross-over design and provided sufficient reporting, we followed the recommendations detailed in Chapter 16.4.5 in the Cochrane Handbook for Systematic Reviews of Interventions (Elbourne 2002; Higgins 2011).

\section{Dealing with missing data}

When we identified missing or unclear data, we contacted the study author directly. We followed Cochrane recommendations when dealing with such data details, as provided in Chapter 16 of the Cochrane Handbook for Systematic Reviews of Interventions (Higgins 2011), and we considered two approaches.

- Analysing only available data.

- Imputing the missing data using replacement values and treating these as if they were observed.

\section{Assessment of heterogeneity}

We followed Cochrane recommendations for assessment of heterogeneity (Deeks 2011). We visually investigated heterogeneity by using forest plots generated via RevMan 5.4 (RevMan 2020). We assessed statistical heterogeneity of treatment effects between pooled trials for each considered outcome by using the $I^{2}$ statistic to quantify the degree of heterogeneity (Higgins 2002). We considered an $\mathrm{I}^{2}>30 \%$ as showing moderate heterogeneity, with an $\mathrm{I}^{2}>75 \%$ signifying significant heterogeneity.

\section{Assessment of reporting biases}

We planned to generate funnel plots and to perform Egger's linear regression tests to investigate reporting biases for considered outcomes when the number of trials included in a single meta-analysis was sufficient (at least 10 trials). We followed recommendations provided in Chapter 10 of the Cochrane Handbook for Systematic Reviews of Interventions (Sterne 2011).

\section{Data synthesis}

If sufficient clinically similar studies were available, we pooled their results in meta-analyses and performed meta-analyses based on ITT analyses when available, according to PD-L1 tumour proportional score (TPS) or PD-L1 expression on tumour cells (TC) or immune cells (IC). Considered PD-L1 categories were as follows: "positive" (PD-L1 TPS $\geq 1 \%$ or TC1-2-3/IC1-2-3), "negative" (PD-L1 TPS $<1 \%$ or TC0/IC0), "high" (PD-L1 TPS $\geq 50 \%$ or PD-L1 TC3/IC3).

We performed meta-analyses according to recommendations given in Chapter 9 of the Cochrane Handbook for Systematic Reviews of Interventions (Higgins 2011). For meta-analyses, we entered data into RevMan 5.4 (RevMan 2020). One review author (RM) entered the data, and a second review author (RF) double-checked the data for accuracy.

We performed random-effects model for all outcomes and fixedeffect model for selected outcomes as sensitivity analyses. 
We applied the inverse-variance method for fixed-effect model for time-to-event outcomes. We applied the Mantel-Haenszel method for dichotomous outcomes and the inverse-variance method for continuous outcomes. We planned to use Peto's odds ratio (OR) method under the fixed-effect model in cases of rare events (Brockhaus 2014). For random-effects model, we applied the DerSimonian and Laird method (DerSimonian 1986).

We followed the GRADE approach when creating our 'Summary of findings' tables, as suggested in Chapters 11 and 12 of the Cochrane Handbook for Systematic Reviews of Interventions (Higgins 2011).

We used the five GRADE considerations to rate the certainty of evidence as 'high', 'moderate', 'low', or 'very low'.

- Risk of bias: serious or very serious.

- Inconsistency: serious or very serious.

- Indirectness: serious or very serious.

- Imprecision: serious or very serious.

- Publication bias: likely or very likely.

We created two 'Summary of findings' tables.

1. Single immune checkpoint inhibitors (ICls) compared to chemotherapy for people with advanced non-small cell lung cancer (NSCLC) (Summary of findings 1 )

2. Combined immune checkpoint inhibitors (ICls) compared to chemotherapy for people with advanced non-small cell lung cancer (NSCLC) (Summary of findings 2)

The following outcomes were included.

- OS

- PFS

- ORR

- AEs: grades 3,4

- HRQoL

\section{Living systematic review considerations}

Whenever we identify new evidence (meaning new studies, data, or information) that is relevant to the review, we will extract the data and assess risk of bias, as appropriate. We will not adjust metaanalyses to account for multiple testing, given that methods related to frequent updating of meta-analyses are under development (Simmonds 2017).

We will wait until accumulating evidence changes one or more of the following components of the review before incorporating it and re-publishing the review.

- Findings of one or more outcomes (e.g. clinically important change in size or direction of effect).

- Credibility (e.g. GRADE rating) of one or more outcomes.

- New settings, populations, interventions, comparisons, or outcomes studied.

\section{Subgroup analysis and investigation of heterogeneity}

We performed subgroup analyses, when data were adequate, to assess the effect on heterogeneity for each of the primary and secondary outcomes on the following subgroups.

- TMB measured on tissue or blood (high versus low). For studies where different analyses of TMB were presented, we chose the TMB assessment and cut-offs with more available data.

- Clinical characteristics such as age, gender, performance status, smoking history, NSCLC histology.

\section{Sensitivity analysis}

We investigated the robustness of review by performing the following sensitivity analyses when appropriate.

- Performing fixed-effect models for selected outcomes.

- Including only 'low risk of bias' outcomes, according to the summary assessment of risk of bias.

- Including or not including results from studies with incomplete data, whether or not the data were imputed.

\section{Methods for future updates}

We will review the scope and methods of this review approximately yearly, or more frequently, if appropriate, in light of potential changes in the topic area or in evidence available for inclusion in the review (e.g. additional comparisons, interventions, or outcomes), or according to newly available review methods.

We will consider each year the necessity for the review to be living by assessing ongoing relevance of the question to decision-makers and by determining whether uncertainty is ongoing in the evidence and whether further relevant research is likely.

\section{RE S U L T S}

\section{Description of studies}

See Characteristics of included studies; Characteristics of excluded studies and Characteristics of ongoing studies.

\section{Results of the search}

This is a living systematic review and its goal is to actively include relevant new evidence when it becomes available. A total of 12,374 records were retrieved from searching the databases electronically up to 21st October 2020. Two thousand, nine hundred and fortyone from CENTRAL, 4886 records from MEDLINE and 4547 from Embase. After removing the duplicate references, 6809 records remained, and three review authors (CM, RM, MI) excluded 6789 records based on screening the titles and abstracts. We selected 20 records that appeared to be relevant on the basis of full-text screening. Among these we identified seven completed studies (Carbone 2017; Hellmann 2018; Herbst 2020; Mok 2019; Reck 2016; Rizvi 2020; Sezer 2020) for inclusion in this review, and eight ongoing studies EMPOWER-Lung 2; eNERGY; IPSOS; JAVELIN Lung 100; KEYNOTE-598;MILES 5; NEPTUNE; PEARL). We excluded the other five studies (Huan 2019; Hui 2017a; Leighl 2019; Mok 2017; Ready 2019) (see Excluded studies and the PRISMA study flow diagram in Figure 1). 
Figure 1. Study flow diagram.

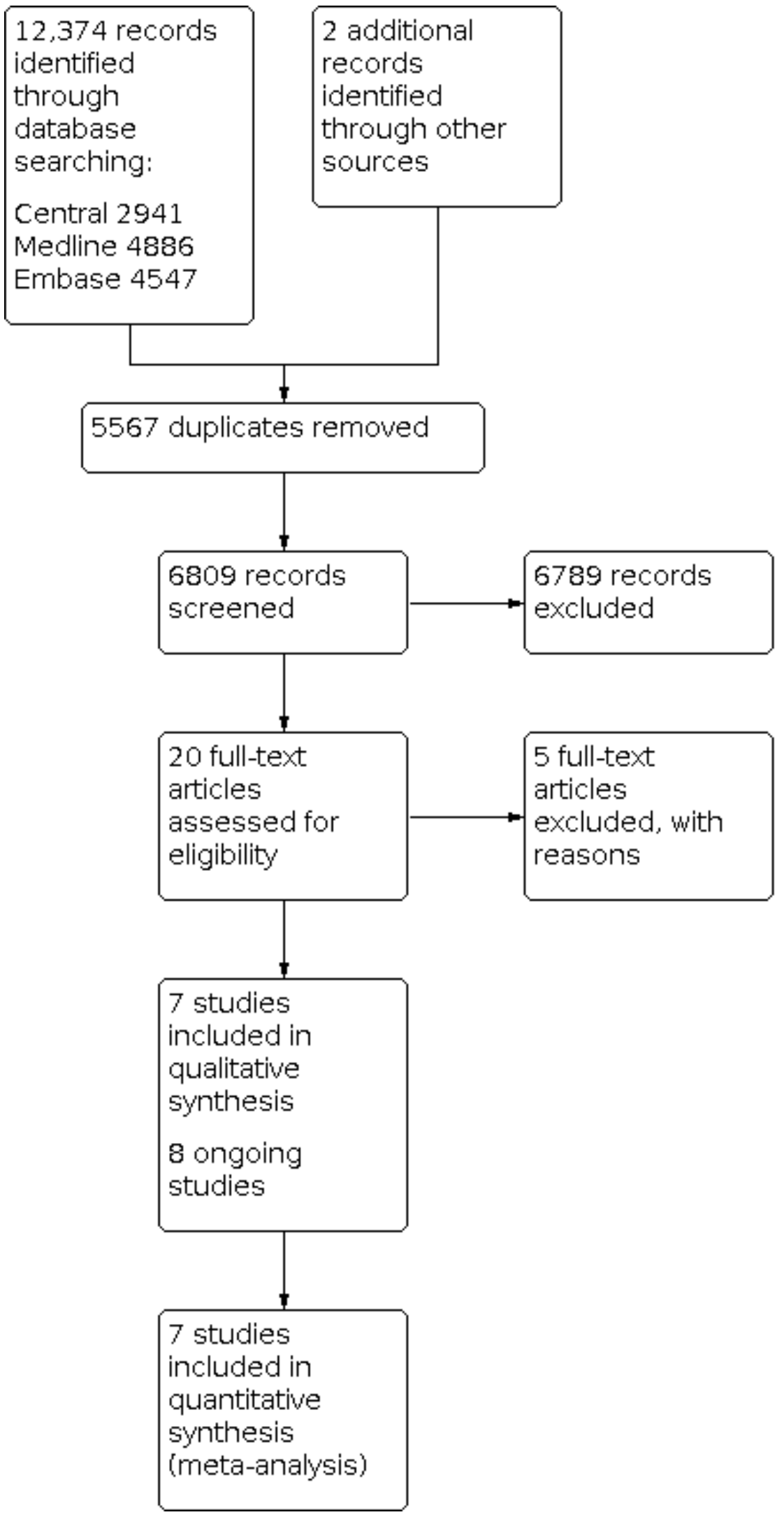




\section{Included studies}

All seven included studies were randomised controlled trials (RCTs) published in English between 2016 and 2020. We divided the included studies by intervention strategies into two types:

- single-agent immune checkpoint inhibitor (ICI) compared with standard chemotherapy;

- combination of $\mathrm{ICl}$ compared with standard chemotherapy.

Six of the seven included studies compared single checkpoint inhibitors versus standard chemotherapy and two studies compared a double checkpoint inhibitor strategy to standard chemotherapy.

\section{Single-agent immune checkpoint inhibitors (ICls) versus standard platinum-based chemotherapy}

We identified six trials comparing a single checkpoint inhibitor agent with a standard chemotherapy for the treatment of NSCLC (Carbone 2017; Herbst 2020; Mok 2019; Reck 2016; Rizvi 2020; Sezer 2020). All six trials were randomised controlled phase 3 trials with an open-label trial design. Overall, 3893 participants with stage IV or recurrent NSCLC were included in these trials.

\section{Population}

All six trials were international multicentre studies involving adults, age 18 or over, with histologically-confirmed stage IV NSCLC who had not received any previous systemic anti-cancer treatment for advanced disease. The inclusion criteria across the trials were similar and all included people with NSCLC without epidermal growth factor receptor (EGFR) mutations or anaplastic lymphoma kinase (ALK) translocations, with Eastern Cooperative Oncology Group (ECOG) performance score (PS) 0-1 and with adequately treated brain metastases. Three trials (Carbone 2017; Herbst 2020; Mok 2019), included participants with PD-L1 positive tumours (PDL1 tumour proportional score (TPS) $\geq 1 \%$ or PDL1 TC $1 / 2 / 3$ or IC 1/2/3). In two trials (Reck 2016; Sezer 2020), only participants with high expression of PD-L1 (TPS $\geq 50 \%$ ) were eligible for inclusion, whereas in Rizvi 2020, participants with negative PD-L1 expression (PD-L1 TPS $<1 \%$ ) were also included.

In Sezer 2020, PD-L1 testing instruction changed in August 2018 and a population with confirmed PD-L1 TPS $\geq 50 \%$ was defined as PDL1 $\geq 50 \%$ ITT population.

The number of randomised participants varied from 305 in Reck 2016 to 1274 in Mok 2019. Trial participants were stratified according to the tumour histology types across all the six trials (Carbone 2017; Herbst 2020; Mok 2019; Reck 2016; Rizvi 2020; Sezer 2020), PD-L1 expression in four trials (Carbone 2017; Herbst 2020; Mok 2019; Rizvi 2020), ECOG PS in three trials (Herbst 2020; Mok 2019; Reck 2016), and the region of enrolment in three trials (Mok 2019; Reck 2016; Sezer 2020). In Carbone 2017, participants were also grouped according to their tumour mutational burden (TMB) level into low- ( 0 to $<100$ mutations), medium- (100 to 242 mutations) and high-burden groups (> 243 mutations).

The proportion of women was lower in the nivolumab group and the proportion of people with a PD-L1 expression level of $50 \%$ or more was also lower in Carbone 2017. In addition, participants in the nivolumab arm had a greater TMB. In Reck 2016, the proportion of people who never smoked was lower in the pembrolizumab arm and the proportion of people with brain metastases was higher in the pembrolizumab arm. In Herbst 2020, Mok 2019, and Sezer 2020, there were no differences in baseline characteristics.

\section{Setting}

All six included trials were multicentre and participants were enrolled from centres in North America, Europe, Asia and Australia.

\section{Intervention}

All included trials compared a single-agent anti-PD-1 or anti-PD-L1 with platinum-based chemotherapy. In Carbone 2017, nivolumab was given at $3 \mathrm{mg} / \mathrm{kg}$ of body weight every two weeks until disease progression, toxicity or withdrawal of consent. In Mok 2019 and Reck 2016, pembrolizumab was given at a flat dose of $200 \mathrm{mg}$ every three weeks for up two years. In Rizvi 2020, durvalumab as monotherapy was given at $20 \mathrm{mg} / \mathrm{kg}$ of body weight every four weeks. In Herbst 2020, atezolizumab was given at 1200 mg every three weeks until disease progression or loss of clinical benefit. In Sezer 2020, cemiplimab was administered at a dose of 350 mg every three weeks until disease progression the maximum duration for 108 weeks.

Cross-over was allowed in Carbone 2017 Reck 2016 and Sezer 2020, but it was not permitted in Herbst 2020, Mok 2019 or Rizvi 2020. In Sezer 2020, participants progressing to cemiplimab had the opportunity to continue anti-PD1 in association with chemotherapy.

\section{Study duration}

Median duration of follow-up was 13.5 months in Carbone 2017, 25.2 months in Reck 2016, 11.2 months in Mok 2019, 30.2 months in Rizvi 2020, 10.8 months in the PD-L1 $\geq 50 \%$ intention-to-treat (IIT) population in Sezer 2020 and 13.4 months for participants with positive (tumour cells (TC) or immune cells (IC) score $\geq 1 \%$ ) PD-L1 in Herbst 2020.

\section{Outcomes}

\section{Primary outcomes}

Overall survival (OS) was the primary outcome in three included trials Herbst 2020, Mok 2019 and Sezer 2020. In Mok 2019, the initial plan was to report OS for participants with PDL1 TPS $\geq$ $50 \%$. However, after trial amendment OS was reported for people with both PD-L1 TPS of $\geq 50 \%$ and $\geq 1 \%$. In addition, in the same trial, OS for participants with PD-L1 TPS $\geq 20 \%$ was also added as a primary outcome. In Rizvi 2020, the primary outcome was OS for durvalumab in monotherapy compared with chemotherapy in patients with PD-L1 $\geq 25 \%$. OS for PD-L1 positive (TC or IC score $\geq$ 1\%) participants was the primary outcome in Herbst 2020.

Progression-free survival (PFS) was the primary outcome in two trials Carbone 2017 and Reck 2016, and was a primary outcome together with OS in Sezer 2020. In Carbone 2017, PFS was reported for participants with PDL1 TPS $\geq 5 \%$. In Reck 2016, PFS was reported for all participants with PDL1 TPS $\geq 50 \%$.

\section{Secondary outcomes}

PFS was the secondary outcome in Carbone 2017 for participants with PD-L1 TPS $\geq 1 \%$, in Mok 2019 for people with PD-L1 TPS $\geq 1 \%$, 
$\geq 20 \%$ and $\geq 50 \%$, in Rizvi 2020 for people with PD-L1 TPS $\geq 25 \%$, and in Herbst 2020 for all PD-L1 positive participants (TC or IC score $\geq 1 \%)$.

OS was a secondary outcome in Carbone 2017 and Reck 2016. OS was reported for people with PD-L1 TPS $\geq 5 \%$ and PD-L1 TPS $\geq 1 \%$ in Carbone 2017 and for participants with PD-L1 TPS $\geq 50 \%$ in Reck 2016.

Objective response rate (ORR) was a secondary endpoint in Carbone 2017 for participants with PD-L1 TPS $\geq 5 \%$, in Reck 2016 for PD-L1 TPS $\geq 50 \%$, in Mok 2019 for people with PD-L1 TPS $\geq 1 \%$, $\geq 20 \%$ and $\geq 50 \%$, in Rizvi 2020 for PD-L1 TPS $\geq 25 \%$ subgroup, in Herbst 2020 for PD-L1 positive participants (TC or IC score $\geq 1 \%$ ) and in Sezer 2020, for PD-L1 TPS $\geq 50 \%$.

ORR, PFS and OS according to TMB status were exploratory analyses in Carbone 2017, Herbst 2020, Mok 2019 and Rizvi 2020.

\section{Combined inhibitors (ICIs) versus standard platinum-based chemotherapy}

We only identified two trials Hellmann 2018 and Rizvi 2020 in which a different checkpoint inhibitor combination was compared with a standard chemotherapy treatment. Both were multicentre randomised controlled phase 3 open-label design trials. Overall, 1910 participants were included in these two studies.

\section{Population}

Adults with histologically-confirmed stage IV NSCLC who had not received previous systemic anticancer treatment for advanced disease were enrolled in both trials (Hellmann 2018; Rizvi 2020). Similarly, both trials involved participants with ECOG PS score 0-1, adequately-treated brain metastases and without EGFR mutations or ALK translocations. However, in part one of Hellmann 2018, only participants with PD-L1 TPS $\geq 1 \%$ were included. On the other hand, the PD-L1 positive status was not an inclusion criteria in Rizvi 2020.

Participants were stratified by tumour-histology types in both trials. Participants were stratified by the PD-L1 expression levels, $<25 \%$ versus $\geq 25 \%$ in Rizvi 2020 . The TMB co-primary survival analysis was conducted in the subgroup of participants who had a TMB of at least 10 mutations per megabase in Hellmann 2018.

People characteristics were well-balanced among the groups in both Hellmann 2018 and Rizvi 2020.

\section{Setting}

Both were multicentre international trials and the recruitment centres were in North America, Europe, Asia and Australia.

\section{Intervention}

Anti-CTLA-4 agents were tested in both trials. The combination of nivolumab and ipilimumab was the intervention arm compared to chemotherapy in Hellmann 2018. The combination of durvalumab and tremelimumab was compared with the standard chemotherapy in Rizvi 2020. Nivolumab was given at $3 \mathrm{mg} / \mathrm{kg}$ of body weight every two weeks until disease progression in combination with ipilimumab at $1 \mathrm{mg} / \mathrm{kg}$ every six weeks for a maximum of four cycles in Hellmann 2018. Durvalumab was given at a dose of $20 \mathrm{mg} / \mathrm{kg}$ every four weeks until disease progression or in association to tremelimumab $1 \mathrm{mg} / \mathrm{kg}$ every four weeks for up to four doses in Rizvi 2020.

Cross-over during the trial was not permitted in both trials.

\section{Study duration}

Median duration of follow up was 30.2 months for people with PDL1 expression $\geq 25 \%$ in Rizvi 2020. The minimum follow-up for OS was 29.3 months in Hellmann 2018.

\section{Outcomes}

Primary outcomes

Primary endpoints were PFS (assessed by BIRC (Blinded, Independent Review Committee)) with nivolumab plus ipilimumab versus chemotherapy in participants with TMB $\geq 10$ mutations per megabase and OS in PD-L1 $\geq 1 \%$ participants in Hellmann 2018 . In Rizvi 2020, primary endpoints were PFS, (according to BIRC), and OS with durvalumab + tremelimumab compared to platinumbased chemotherapy in patients with PD-L1 $\geq 25 \%$.

\section{Secondary outcomes}

Secondary endpoints included PFS among participants with TMB $\geq 10$ mutations per megabase and a PD-L1 $\geq 1 \%$ and OS among people with $T M B \geq 10$ mutations per megabase in Hellmann 2018. In Rizvi 2020, key secondary endpoints were PFS and ORR with durvalumab + tremelimumab compared to platinum-based chemotherapy in people with PD-L1 $\geq 25 \%$, in participants with PD$\mathrm{L} 1$ expression $\geq 1 \%$ and in the overall population. ORR in the overall population was a secondary endpoint for Hellmann 2018.

OS, PFS and ORR by TMB were exploratory analyses in Rizvi 2020.

\section{Excluded studies}

We excluded five studies for the following reasons (see Characteristics of excluded studies).

- Wrong study design: three studies:

* Huan 2019 because it is not an RCT;

* Hui 2017a and Leighl 2019 refer to the same study: KEYNOTE 001 which is a multi-cohort phase 1 trial.

- Wrong intervention: two studies:

* Mok 2017: evaluates ICI plus chemotherapy;

* Ready 2019: does not compare ICI with chemotherapy.

\section{Risk of bias in included studies}

Figure 2 and Figure 3 are a visual representative of the 'Risk of bias' assessment across all included trials and also for each individual domain in the included trials. See Characteristics of included studies section 'Risk of bias' table for further explanations about the bias identified for each domain within each included studies. 
Figure 2. 'Risk of bias' graph: review authors' judgements about each risk of bias item presented as percentages across all included studies.

Random sequence generation (selection bias)

Allocation concealment (selection bias)

Blinding of participants and personnel (performance bias): All outcomes

Blinding of outcome assessment (detection bias): All outcomes Incomplete outcome data (attrition bias): All outcomes

Selective reporting (reporting bias)

Other bias
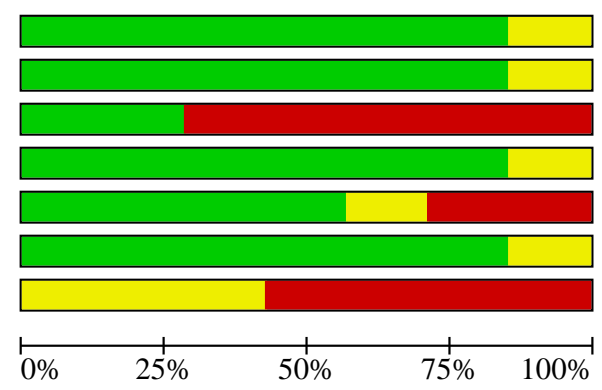

$\square$ Low risk of bias $\quad \square$ Unclear risk of bias

High risk of bias 
Figure 3. 'Risk of bias' summary: review authors' judgements about each risk of bias item for each included study.

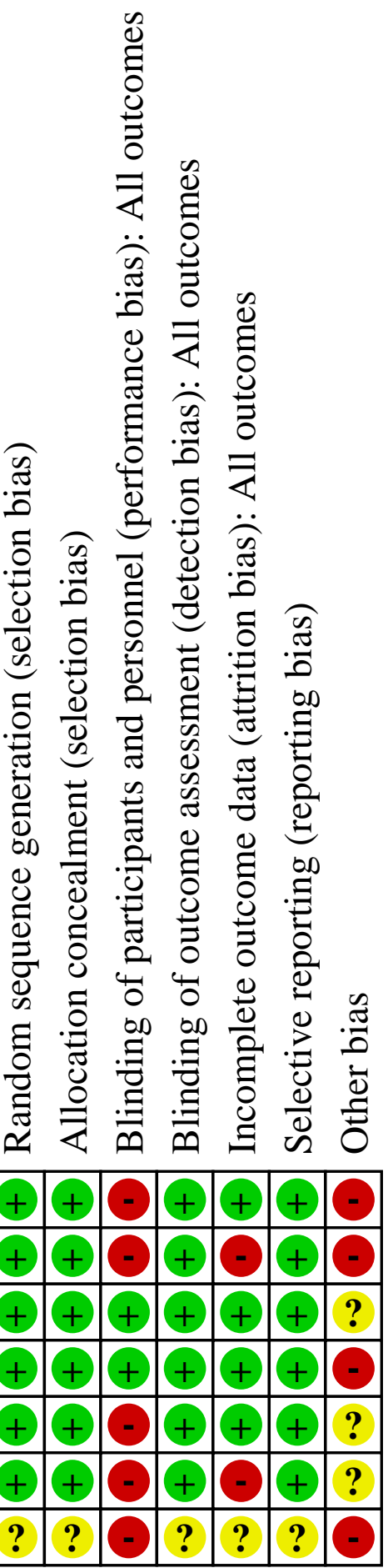


We assessed the risk of bias of five included studies using information published in full papers, one trial (Sezer 2020) was only available as abstract. The overall risk of bias was generally low or unclear, with much of the methodological information confirmed through a direct checking of the trial protocols to support the published information and to clarify the reasons for our rating.

\section{Allocation}

Six included trials (Carbone 2017; Hellmann 2018; Herbst 2020; Mok 2019; Reck 2016; Rizvi 2020) were at low risk of selection bias because of adequate methods of sequence generation and allocation concealment. Methods of randomisation and allocation concealment were stated across all the included trials. We rated Sezer 2020 as unclear as the method of randomisation and assignment to treatment were not reported.

\section{Blinding}

\section{Blinding of participants and personnel (performance bias)}

All seven included studies had an open-label design being at potential risk of performance bias. However only Carbone 2017, Hellmann 2018; Reck 2016, Rizvi 2020 and Sezer 2020 where PFS was a primary endpoint were at high risk of performance bias due to absence of blinding of participants and personnel, whereas in Herbst 2020 and Mok 2019, where the primary endpoint was OS, performance bias influenced only results of secondary or exploratory outcomes.

\section{Blinding of outcome assessors (detection bias)}

Five included trials (Carbone 2017; Hellmann 2018; Mok 2019; Reck 2016; Rizvi 2020), were at low risk of detection bias because outcome assessors were blinded. In one study (Herbst 2020), the primary outcome measured was OS, therefore, lack of blinding of the outcome assessors had no influence. In Sezer 2020, relevant information regarding blinding of outcome assessors was missing.

\section{Incomplete outcome data}

Four trials (Carbone 2017; Herbst 2020; Mok 2019; Reck 2016) were at low risk of attrition bias, as no participants were lost to follow-up and the total number of participants included in each outcome was reported. Two trials (Hellmann 2018; Rizvi 2020) were at high risk of attrition bias. In Hellmann 2018) only 229 (13\%) out of 1739 initially randomised participants were included in the analysis of the trial primary outcome. In Rizvi 2020, the primary analysis population for the study was amended to include only people with PD-L1 expression $\geq 25 \%$, therefore only $488(44 \%)$ of 1118 randomised people were included in the analysis of the trial primary outcome. In Sezer 2020, the information relevant to the number of dropouts was missing for most of the outcome, therefore we rated this domain as 'unclear'.

\section{Selective reporting} Reck 2016; Rizvi 2020) were at low risk of reporting bias as all prespecified outcomes were reported. Sezer 2020, was only available as a conference presentation with not enough details to make a judgment and risk of reporting bias was considered unclear.
Six trials (Carbone 2017; Hellmann 2018; Herbst 2020; Mok 2019;

\section{Other potential sources of bias}

All trials were at unclear risk of other source of bias because some authors had declared personal fees or other support from the pharmaceutical companies conducting the trials and it was impossible to know how these conflicts of interests have biased data collection and analysis. Furthermore, Carbone 2017, was at high risk of other source of bias because of some baseline differences between the groups. In the nivolumab group, the percentage of women was lower than that in the chemotherapy group (32\% versus. $45 \%$ ), as was the percentage of participants with a PD-L1 expression level of $50 \%$ or more (32\% versus $47 \%$ ); the percentage of participants with liver metastases was slightly higher in the nivolumab group (20\% versus. $13 \%$ ). In addition, people in the nivolumab group had a lower tumour mutational burden than those in the chemotherapy group (30\% versus 39\%). Mok 2019 was at high risk of other source of bias due to the inclusion of a new PD-L1 TPS cut-off (20\%) and the change of primary and secondary endpoint according to a new PD-L1 TPS categorisation $(\geq 1 \%$, $\geq$ $20 \%, \geq 50 \%$ ). Hellmann 2018, was at high risk of other source of bias due to the amendment including TMB as a new biomarker and the modification of primary and secondary endpoint accordingly to TMB levels. We rated Sezer 2020, at high risk of other sources of bias because although PD-L1 $\geq 50 \%$ was an inclusion criteria, 235 participants were retested and 88 of them had a confirmed PD-L1 TPS $\geq 50 \%$ being included in the PD-L1 $\geq 50 \%$ ITT population.

\section{Publication bias}

We did not have sufficient data to provide a funnel plot, nor to perform the Egger test. Publication bias was assessed only for the six trials testing single-agent $\mathrm{ICl}$ versus platinum-based chemotherapy, because for the comparison double $\mathrm{ICI}$ versus chemotherapy only two studies were available. Publication bias is unlikely to have occurred particularly for the PD-L1 $\geq 1 \%$ category. In fact, trials comparing single-agent $\mathrm{ICl}$ versus platinum-based chemotherapy found mainly unfavourable results or no difference for the experimental arm (single-agent $\mathrm{ICl}$ ) compared to control arm (chemotherapy) for the PD-L $1 \geq 1 \%$ subgroup.

\section{Effects of interventions}

See: Summary of findings 1 Single-immune checkpoint inhibitors (ICls) compared to chemotherapy for people with advanced nonsmall cell lung cancer (NSCLC); Summary of findings 2 Combined immune checkpoint inhibitors (ICls) compared to chemotherapy for people with advanced non-small cell lung cancer (NSCLC)

\section{Comparison 1: First-line single-agent immune checkpoint inhibitor $(\mathrm{ICl})$ versus platinum-based chemotherapy}

\section{Primary outcome - Overall survival (OS)}

- a. Main analysis: OS and PDL-1 expressions

Information on OS was provided by all seven included trials comparing single-agent immune checkpoint inhibitors (ICls) (nivolumab, pembrolizumab durvalumab, atezolizumab, cemiplimab) to standard platinum-based chemotherapy (Carbone 2017; Mok 2019; Reck 2016; Rizvi 2020; Sezer 2020; Herbst 2020). Rizvi 2020, reported OS data for participants with negative PDL1 expression $<1 \%$ as well as PD-L1 level $\geq 1 \%$. Carbone 2017; Mok 2019 and Herbst 2020 provided data for PD-L1 at $\geq 1 \%$ and $\geq 50 \%$,whereas, both Reck 2016 and Sezer 2020 only included participants with PD-L1 $1250 \%$.

Single or combined immune checkpoint inhibitors compared to first-line platinum-based chemotherapy with or without bevacizumab for 17

Copyright (c) 2020 The Cochrane Collaboration. Published by John Wiley \& Sons, Ltd. 
PD-L1 expression < 1\% (negative PD-L1): one trial only (Rizvi 2020) reported data for this subgroup as negative PD-L1 expression was an exclusion criterion for the other five trials. There was no evidence of a difference in OS between $\mathrm{ICl}$ single-agent (durvalumab) and standard platinum-based chemotherapy (hazard ratio (HR): 1.18, 95\% confidence interval (CI) 0.86 to $1.61,1 \mathrm{RCT}, 178$ participants) Analysis 1.1 ; Figure 4

Figure 4. Forest plot of comparison: Single Immuno versus Chemotherapy, outcome: 4.1 Overall survival by PD-L1 expression.

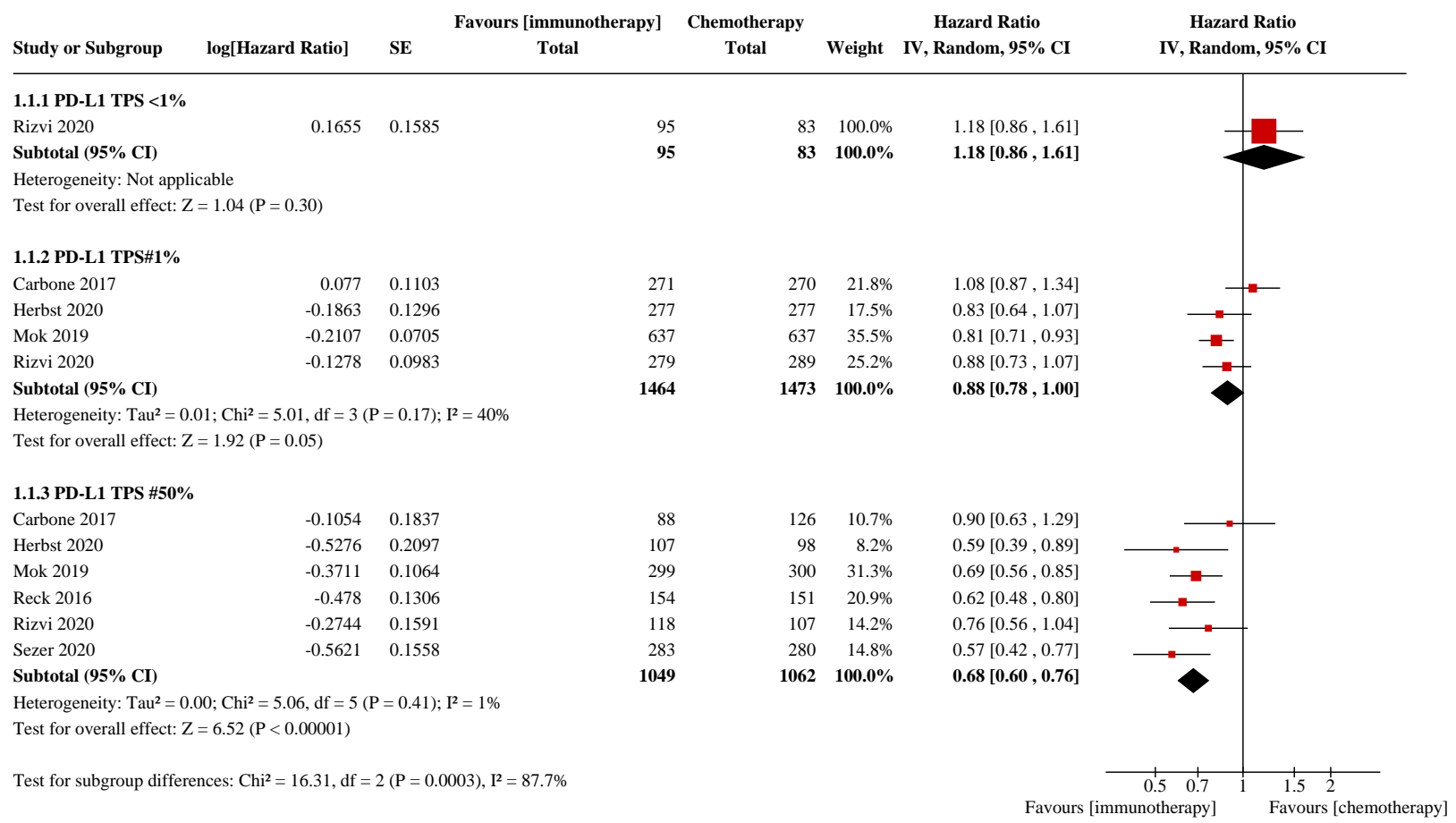

PD-L1 expression $\geq 1 \%$ (positive PD-L1): four trials (Carbone 2017; Mok 2019; Rizvi 2020; Herbst 2020) provided data. There was no evidence of a difference between participants receiving singleagent $\mathrm{ICl}$ or platinum-based chemotherapy (HR $0.88,95 \% \mathrm{Cl} 0.78$ to $1.00, \mathrm{P}=0.05, \mathrm{I}^{2}=40 \%, 4 \mathrm{RCTs}, 2937$ participants) Analysis 1.1 ; Figure 4. However, a considerable level of heterogeneity occurred, while excluding Carbone 2017 and pooling data from the remaining three trials (Mok 2019; Reck 2016; Herbst 2020) showed a difference in favour of single-agent $\mathrm{ICI}$ group with no heterogeneity ( $\mathrm{HR} 0.83,95 \% \mathrm{Cl} 0.75$ to $0.92, \mathrm{P}=0.0005, \mathrm{I}^{2}=0 \%, 4 \mathrm{RCTs}, 2937$ participants) (analysis not shown). A sensitivity analysis applying a fixed-effect model also showed evidence of a difference in favour of the group who received single-agent $\mathrm{ICl}$ compared to platinumbased chemotherapy (HR: $0.87,95 \% \mathrm{Cl} 0.80$ to $0.96, \mathrm{P}=0.004, \mathrm{I}^{2}=$ 40\%, 4 RCTs, 2937 participants) (analysis not shown).

PD-L1 expression $\mathbf{2 5 0 \%}$ (high PD-L1): All six trials (Carbone 2017; Mok 2019; Reck 2016; Rizvi 2020; Sezer 2020; Herbst 2020) reported results. There was probably evidence of a difference favouring the group who received single-agent $\mathrm{ICl}$ compared to platinum-based chemotherapy (HR: $0.68,95 \% \mathrm{Cl} 0.60$ to $0.76, \mathrm{P}<0.00001 ; \mathrm{I}^{2}=1 \%$, 6 RCTs, 2111 participants; moderate certainty of evidence) Analysis 1.1; Figure 4. The certainty for this outcomes was downgraded one level due to study limitations.
There was evidence of a difference between subgroups (PD-L1 negative, positive and high) (Test for subgroup differences: $\mathrm{Chi}^{2}=$ $\left.16.31, \mathrm{df}=2(\mathrm{P}=0.0003), \mathrm{I}^{2}=87.7 \%\right)$, Analysis 1.1; Figure 4 .

- b. Subgroup analyses:

\section{- i. OS and Tumour Mutational Burden (TMB)}

TMB was assessed on tissue in Carbone 2017 and Mok 2019 and thresholds to define high TMB were $>243$ mut/exome (Carbone 2017) and >175 mut/exome (Mok 2019), respectively. In both Rizvi 2020 and Herbst 2020, TMB was assessed on plasma cell-free circulating tumour DNA and cut off to define high TMB was $\geq 20$ Mut/ $\mathrm{Mb}$.

Four trials (Carbone 2017; Mok 2019; Rizvi 2020, Herbst 2020) reported OS results according to two TMB categories, low and high.

TMB - Low, there was no evidence of a difference in OS between single-agent $\mathrm{ICl}$ and platinum-based chemotherapy was reported (HR: $1.01,95 \% \mathrm{Cl} 0.88$ to $1.15, \mathrm{P}=0.92, \mathrm{I}^{2}=0 \%, 4$ RCTs, 1380 participants). Analysis 1.2 ; Figure 5 
Figure 5. Forest plot of comparison: Single Immuno versus Chemotherapy, outcome: 4.4 Overall Survival by Tumor Mutational Burden.

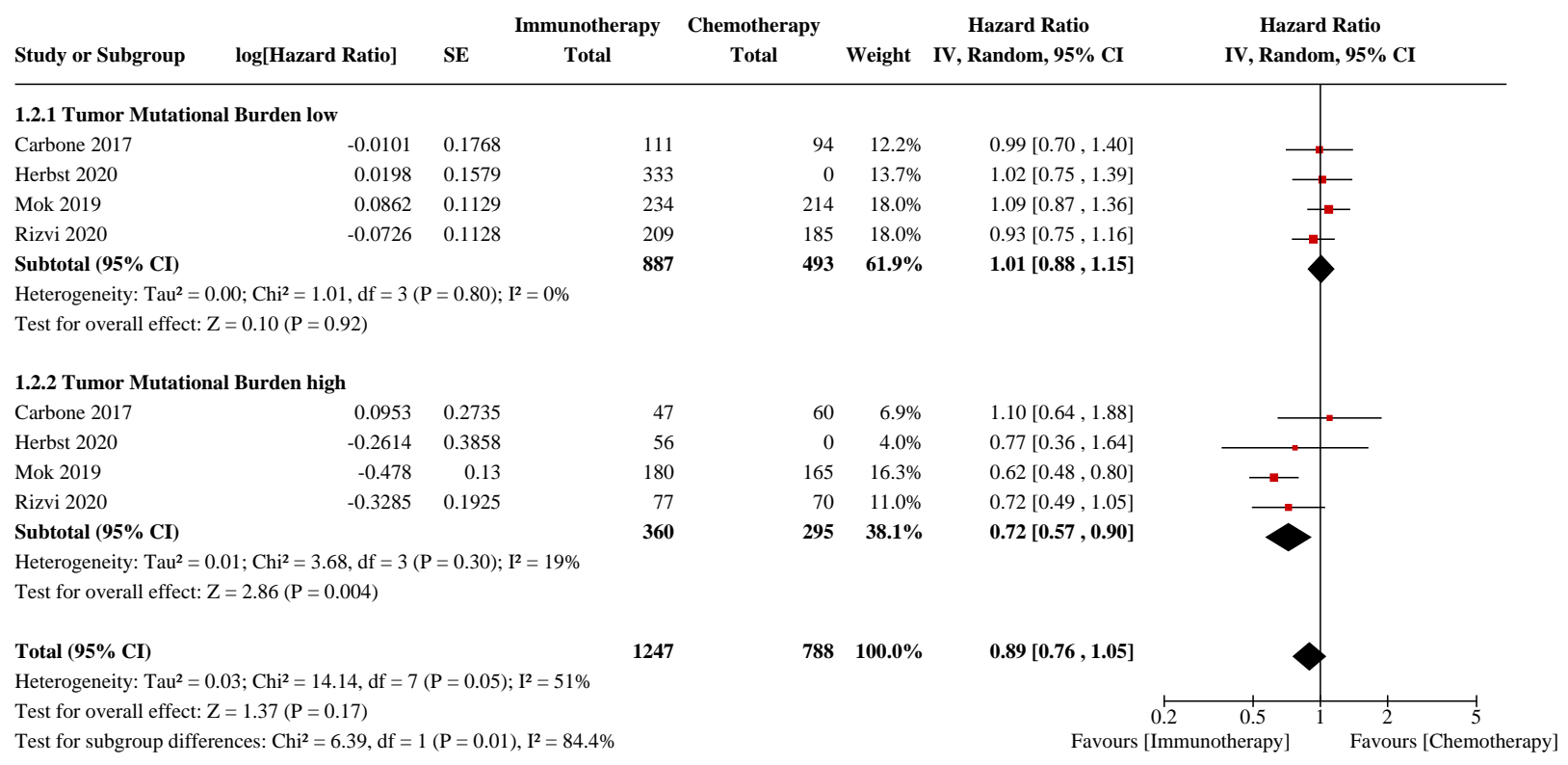

TMB - High, there was evidence of a difference in favour of single-agent $\mathrm{ICl}$ recipients when compared with platinum-based chemotherapy (HR: $0.72,95 \% \mathrm{Cl} 0.57$ to $0.90, \mathrm{P}=0.004, \mathrm{I}^{2}=, 19 \%, 4$ RCTs, 655 participants) Analysis 1.2 ; Figure 5.

Although the pooled survival data showed no evidence of a difference between participants receiving single-agent $\mathrm{ICl}$ or platinum-based chemotherapy, $(\mathrm{HR} 0.89,95 \% \mathrm{Cl} 0.76$,to $1.05, \mathrm{P}=$ $0.17,1^{2}=51 \%, 4$ RCTs, 2035) the test for subgroup differences showed there was evidence of a difference according to TMB-High and TMB-Low categories (Test for subgroup differences: $\mathrm{Chi}^{2}=6.39$, $\left.\mathrm{df}=1(\mathrm{P}=0.01), \mathrm{I}^{2}=84.4 \%\right)$ Analysis 1.2 ; Figure 5.

\section{- ii. OS by age and PD-L1 expression $\geq 1 \%$}

Two trials (Carbone 2017, Mok 2019) provided data for people $<65$ and $\geq 65$ years old and PD-L1 expression $\geq 1 \%$.

Age $<\mathbf{6 5}$ years and PD-L1 expression $\geq 1 \%$. There was no evidence of a difference in OS among $<65$ years old participants who received single-agent $\mathrm{ICl}$ compared to those who randomised to platinumbased chemotherapy (HR: $0.93,95 \% \mathrm{Cl} 0.68$ to $1.29, \mathrm{P}=0.68, \mathrm{I}^{2}=$ 69\%, 2 RCTs, 988 participant) Analysis 1.3.

Age $\geq \mathbf{6 5}$ years and PD-L1 expression $\geq \mathbf{1} \%$. There was no evidence of a difference in OS among $\geq 65$ years old people who received single-agent $\mathrm{ICl}$ compared to platinum-based chemotherapy (HR: $0.90,95 \% \mathrm{Cl} 0.72$ to $1.13, \mathrm{P}=0.36, \mathrm{I}=37 \% 2 \mathrm{RCTs}, 827$ participants) Analysis 1.3.

The pooled survival data showed no evidence of a difference in OS for people who received single-agent $\mathrm{ICl}$ compared to platinumbased chemotherapy (HR 0.90, 95\% $\mathrm{Cl} 0.78$ to $1.03, \mathrm{P}=0.20,12=38 \%$, 2 RCTs, 1815 participants) Analysis 1.5. When applying a fixed-effect there was a difference favouring the single-agent $\mathrm{ICl}$ group without affecting the heterogeneity level (HR $0.89,95 \% \mathrm{Cl} 0.79$ to $1.00 ; \mathrm{P}=$ $0.04,1^{2}=38 \%, 2$ RCTS, participants $=1815$ ) (analysis not shown).
However, there was no evidence of a difference between people $<65$ and $\geq 65$ years old, who received the single-agent $\mathrm{ICl}$ or the standard platinum-based chemotherapy either applying a randomeffects model (Test for subgroup differences: $\mathrm{Chi}^{2}=0.04, \mathrm{df}=1(\mathrm{P}=$ $0.85),\left.\right|^{2}=0 \%$ ) or a fixed-effect model (Test for subgroup differences: $\left.\mathrm{Chi}^{2}=0.00, \mathrm{df}=1,(\mathrm{P}=0.98), \mathrm{I}^{2}=0 \%\right)$ (analysis not shown), Analysis 1.3 .

\section{- iii. OS by age and PD-L1 expression $\geq 50 \%$}

Three trials (Mok 2019; Reck 2016; Herbst 2020) provided data for this outcome.

Age < 65 years and PD-L1 expression $\geq 50 \%$. There was evidence of a difference in OS favouring among people $<65$ years old who received single-agent $\mathrm{ICl}$ compared to platinum-based chemotherapy (HR: $0.72,95 \% \mathrm{Cl} 0.57$ to $0.90, \mathrm{P}=0.004, \mathrm{l}=0 \%, 3$ RCTs, 571 participants) Analysis 1.4

Age $\geq \mathbf{6 5}$ years and PD-L1 expression $\geq \mathbf{5 0} \%$. There was evidence of a difference in OS favouring $\geq 65$ years old people who received single-agent $\mathrm{ICl}$ compared to platinum-based chemotherapy (HR: $0.60,95 \% \mathrm{Cl} 0.47$ to $0.78, \mathrm{P}<0.0001, \mathrm{I} 2=0 \%, 2 \mathrm{RCTs}, 435$ participants) Analysis 1.4

The pooled survival data showed evidence of a difference in OS favouring participants receiving single-agent $\mathrm{ICl}$ compared to platinum-based chemotherapy (HR: $0.67,95 \% \mathrm{Cl} 0.56$ to 0.79 , P $<0.00001,12=0 \%, 3$ RCTs, 1006 participants) Analysis 1.4. No difference according to age subgroups (people $<65$ and $\geq 65$ years old with PD-L1 expression $\geq 50 \%$ ) was observed (Test for subgroup differences: $\left.\mathrm{Chi}^{2}=1.06, \mathrm{df}=1(\mathrm{P}=0.30), \mathrm{I}^{2}=5.6 \%\right)$. Analysis 1.4 .

\section{- iv. OS by gender and PD-L1 expression $\geq 1 \%$}

Single or combined immune checkpoint inhibitors compared to first-line platinum-based chemotherapy with or without bevacizumab for 
Four trials (Carbone 2017; Mok 2019; Reck 2016; Herbst 2020) provided data for overall survival outcome according to PD-L1 levels and gender.

Data were reported by Carbone 2017 and Mok 2019.

\section{Males with PD-L1 expression $\geq 1 \%$.}

There was no evidence of a difference in OS among males participants receiving single-agent $\mathrm{ICl}$ compared to platinumbased chemotherapy ( HR; $0.86,95 \% \mathrm{Cl} 0.71$ to $1.03, \mathrm{P}=0.09, \mathrm{I}^{2}=$ $33 \%, 2$ RCTS, 1234 participants) Analysis 1.5. When applying a fixedeffect model there was a difference in favour of the single-agent $\mathrm{ICl}$ group with no changes in the heterogeneity level (HR: $0.84,95 \% \mathrm{Cl}$ 0.74 to $0.97, \mathrm{P}=0.02, \mathrm{I} 2=33 \%, 2$ RCTS, 1234 participants).

\section{Females with PD-L1 expression $\geq 1 \%$.}

There was no evidence of a difference in OS among females who received single-agent $\mathrm{ICl}$ compared to platinum-based chemotherapy (HR: $0.98,95 \% \mathrm{Cl} 0.77$ to $1.25, \mathrm{P}=0.88, \mathrm{I}^{2}=17 \%, 2$ RCTs, 581 participants) Analysis 1.5

Moreover, there was no evidence of a difference between subgroups (Test for subgroup differences: $\mathrm{Chi}^{2}=0.78, \mathrm{df}=1(\mathrm{P}=$ $\left.0.38), I^{2}=0 \%\right)$.

The overall pooled survival data showed no evidence of a difference in OS among males and females with PD-L1 level $\geq 1 \%$ who received single-agent $\mathrm{ICl}$ compared to platinum-based chemotherapy (HR: $0.9095 \% \mathrm{Cl} 0.78$ to $1.03, \mathrm{P}=0.12, \mathrm{I} 2=23 \%, 2 \mathrm{RCTs}, 1815$ participants) Analysis 1.5. When applying a fixed-effect model a difference in OS was observed favouring the single-agent $\mathrm{ICl}$ group compared to platinum-based chemotherapy (HR: $0.8895 \% \mathrm{Cl} 0.78$ to $0.99, \mathrm{P}=$ $0.03, I^{2}=23 \%, 2$ RCTs, 1815 participants).

However, no difference according to gender subgroups (male or female with PD-L1 expression $\geq 1 \%$ ) was observed applying either a random-effects model (Test for subgroup differences: $\mathrm{Chi}^{2}=0.78$, $\mathrm{df}=1, \mathrm{P}=0.38, \mathrm{I}^{2}=0 \%$ ) or a fixed-effect model (Test for subgroup differences: $\mathrm{Chi}^{2}=1.19, \mathrm{df}=1, \mathrm{P}=0.27, \mathrm{I}^{2}=16.3 \%$ ) (analysis not shown) Analysis 1.5.

\section{- v. OS by gender and PD-L1 expression $\geq 50 \%$}

Three trials (Mok 2019; Reck 2016; Herbst 2020) reported OS data by gender.

Males with PD-L1 expression $\geq \mathbf{5 0} \%$. There was evidence of a difference in OS among male participants who received singleagent $\mathrm{ICl}$ compared to platinum-based chemotherapy (HR: 0.62 , $95 \% \mathrm{Cl} 0.52$ to $0.76, \mathrm{P}<0.00001$. $\mathrm{I}^{2}=0 \%, 3 \mathrm{RCTs}, 745$ participants) Analysis 1.6

Females with PD-L1 expression $\mathbf{2} \mathbf{5 0} \%$. There was no evidence of a difference for female participants who received single-agent $\mathrm{ICI}$ compared to platinum-based chemotherapy (HR: $0.81,95 \% \mathrm{Cl} 0.61$ to $1.08, \mathrm{P}=0.15, \mathrm{I}^{2}=0 \%, 3 \mathrm{RCTs}, 363$ participants) Analysis 1.6.

The overall pooled results showed evidence of a difference in OS favouring the group who received single-agent $\mathrm{ICI}$ compared to platinum-based chemotherapy $(\mathrm{HR} 0.68,95 \% \mathrm{Cl} 0.58$ to $0.79, \mathrm{P}<$ $0.00001, \mathrm{I}^{2}=0 \%$, 3RCTs, 1108 participants) Analysis 1.6.
Furthermore, there was no evidence of a difference between the two subgroups (Test for subgroup differences: $\mathrm{Chi}^{2}=2.17, \mathrm{df}=1(\mathrm{P}$ $\left.=0.14), I^{2}=54.0 \%\right)$ Analysis 1.6.

\section{- vi. OS by smoking status and PD-L1 expression $\geq 1 \%$}

Two trials (Carbone 2017 and Mok 2019) provided data for this outcome. We reported the OS and the smoking status results by the following categories.

Never smoked and PD-L1 expression $\geq \mathbf{1} \%$. There was no evidence of a difference between never smokers who received single-agent $\mathrm{ICl}$ compared to platinum- based chemotherapy (HR: $1.00,95 \% \mathrm{Cl}$ 0.76 to $1.33, P=0.98$, fixed-effect model or random-effects model, $\mathrm{I} 2=0 \%, 2 \mathrm{RCTs}, 341$ participants) Analysis 1.7

Former smokers and PD-L1 expression $\geq 1 \%$. There was no evidence of a difference in OS between former smokers who received single-agent $\mathrm{ICl}$ compared to platinum-based chemotherapy HR: $0.87,95 \% \mathrm{Cl} 0.57$ to $1.33, \mathrm{P}=0.52, \mathrm{~L}=85 \%$, 2 RCTs, 1089 participants) Analysis 1.7 However, when applying a fixed-effect model there was a difference favouring the singleagent ICI group with no changes in the heterogeneity level (HR: 0.83 , $95 \% \mathrm{Cl} 0.71$ to $0.96, \mathrm{P}=0.01, \mathrm{l} 2=85 \%, 2 \mathrm{RCTs}, 1089$ participants) (analysis not shown).

Current smokers and PD-L1 expression $\geq 1 \%$. There was no evidence of a difference in OS between current smokers who received single-agent $\mathrm{ICl}$ compared to standard chemotherapy (HR: $0.98,95 \% \mathrm{Cl} 0.75$ to $1.27, \mathrm{P}=0.85, \mathrm{I}=0 \%, 2 \mathrm{RCTs}, 378$ participants) Analysis 1.7

The pooled data showed no evidence of a difference between people receiving single-agent $\mathrm{ICl}$ or platinum-based chemotherapy for people with PD-L1 $\geq 1 \%$ regardless of their smoking status (HR: $0.92,95 \% \mathrm{Cl} 0.78$ to $1.10, \mathrm{P}=0.38, \mathrm{I}^{2}=44 \%, 2$ RCTs, 1808 participants), Analysis 1.7

Applying a fixed-effect model did not alter the level of heterogeneity nor the overall effect (HR 0.89, 95\% Cl 0.79 to $1.00 ; \mathrm{I}^{2}=44 \%, 2$ RCTs, participants $=1808$ ) (analysis not shown). However, when applying a fixed-effect model and excluding Carbone 2017 from the "former smokers" subgroup not only reduced the heterogeneity to $12=32 \%$, but it also showed a difference in OS favouring the single-agent $\mathrm{ICI}$ group regardless of the smoking status (HR: $0.84,95 \% \mathrm{Cl} 0.73$ to $0.96 ; 1^{2}=32 \%, P=0.01,2$ RCTs, participants $=1440$ ) (analysis not shown).

There was no evidence of a difference between smoking status subgroups either when applying a random-effects model (Test for subgroup differences: $\left.\mathrm{Chi}^{2}=0.31, \mathrm{df}=2(\mathrm{P}=0.86), \mathrm{I}^{2}=0 \%\right)$ Analysis 1.7, or a fixed-effect model (Test for subgroup differences: $\mathrm{Chi}^{2}=$ $\left.2.09, \mathrm{df}=2(\mathrm{P}=0.35) \mathrm{I}^{2}=4.1 \%\right)$ and also after exclusion of Carbone 2017 from former smokers subgroup (Test for subgroup differences: $\left.\mathrm{Chi}^{2}=5.75, \mathrm{df}=2(\mathrm{P}=0.06) \mathrm{I}^{2}=65.2 \%\right)$ (analysis not shown). .

\section{- vii. OS by smoking status and PD-L1 expression $\geq 50 \%$}

Three trials (Mok 2019; Reck 2016; Herbst 2020) contributed to this outcome.

Never smokers and PD-L1 expression $\geq \mathbf{5 0 \%}$. There was no evidence of a difference in OS between people who never 
smoked who received single-agent $\mathrm{ICl}$ compared to platinumbased chemotherapy (HR: $1.18,95 \% \mathrm{Cl} 0.78$ to $1.79, \mathrm{P}=0.44, \mathrm{I}^{2}=0 \%$, 3 RCTs, 179 participants), Analysis 1.8 .

Former smokers and PD-L1 expression $\geq \mathbf{5 0 \%}$. There was evidence of a difference in OS favouring the former smoker group who received single-agent $\mathrm{ICl}$ compared to platinum-based chemotherapy (HR: $0.60,95 \% \mathrm{Cl} 0.49$ to $0.73, \mathrm{P}<0.00001, \mathrm{I} 2=0 \%, 3$ RCTs, 700 participants) Analysis 1.8 .

Current smokers and PD-L1 expression $\geq \mathbf{5 0 \%}$. There was evidence of a difference in OS favouring the current smoker group who received single-agent $\mathrm{ICl}$ compared to platinum-based chemotherapy (HR: $0.65,95 \% \mathrm{Cl} 0.44$ to $0.97, \mathrm{P}=0.04 \mathrm{l} 2=11 \%, 3$ RCTs, 230 participants), Analysis 1.8.

The pooled results showed a difference in OS between participants with PD-L1 expression $\geq 50 \%$ who received single-agent $\mathrm{ICl}$ compared to platinum-based chemotherapy regardless of their smoking status (HR: $0.70,95 \% \mathrm{Cl} 0.56$ to $0.86, \mathrm{P}=0.001, \mathrm{I}=29 \%$, 3 RCTs, 1109 participants), Analysis 1.8. In addition, there was evidence of a difference between smoking status subgroups (Test for subgroup differences: $\mathrm{Chi}^{2}=8.27, \mathrm{df}=2, \mathrm{P}=0.02, \mathrm{I}^{2}=75.8 \%$ ) Analysis 1.8.

\section{- viii. OS by ECOG PS and PD-L1 expression $\geq 1 \%$}

Two trials (Carbone 2017; Mok 2019) provided OS data according to ECOG PS and PD-L1 expression, $\geq 1 \%$.

ECOG PS 0 and PD-L1 expression $\geq 1 \%$.. There was no evidence of a difference in OS between ECOG PS 0 people who received singleagent $\mathrm{ICl}$ compared to platinum-based chemotherapy (HR: 0.90, $95 \% \mathrm{Cl} 0.63$ to $1.29, \mathrm{P}=0.57, \mathrm{l}=50 \%, 2 \mathrm{RCTs}, 568$ participants) Analysis 1.9.

ECOG PS 1 and PD-L1 expression $>1 \%$. There was no evidence of a difference in OS between ECOG PS 1 participant who received single-agent $\mathrm{ICl}$ compared to platinum-based chemotherapy (HR: $0.90,95 \% \mathrm{Cl} 0.74,1.09, \mathrm{P}=0.29, \mathrm{I}^{2}=43 \%, 2 \mathrm{RCTs}, 1246$ participants) Analysis 1.9.

The pooled results showed no difference in OS between people receiving single-agent $\mathrm{ICl}$ or platinum-based chemotherapy (HR: $0.89,95 \% \mathrm{Cl} 0.77$ to $1.03, \mathrm{P}=0.11, \mathrm{I} 2=20 \%, 2 \mathrm{RCTs}, 1814$ participants) Analysis 1.9. When applying a fixed-effect model there was a difference favouring single-agent $\mathrm{ICl}$ compared to platinum-based chemotherapy regardless ECOG PS in people with PD-L1 expression $>1 \%$ (HR: $0.88,95 \% \mathrm{Cl} 0.78$ to $1.00, \mathrm{P}=0.04, \mathrm{I}^{2}=20 \%, 2 \mathrm{RCTs}, 1814$ participants) (analysis not shown). There was also no evidence of a difference between the two ECOG PS subgroups either by randomeffects model (Test for subgroup differences: $\mathrm{Chi}^{2}=0.00, \mathrm{df}=1, \mathrm{P}=$ $0.98, \mathrm{I}^{2}=0 \%$ ) Analysis 1.9 or fixed-effect model (Test for subgroup differences: $\mathrm{Chi}^{2}=0.00, \mathrm{df}=1, \mathrm{P}=1.00, \mathrm{I}^{2}=0 \%$ ) (analysis not shown).

\section{- ix. OS by ECOG PS and PD-L1 expression $\geq 50 \%$}

Three trials provided data for this outcome (Herbst 2020 Mok 2019; Reck 2016).

ECOG PS 0 and PD-L1 expression $\geq \mathbf{5 0 \%}$. There was a evidence of a difference in OS favouring the single-agent $\mathrm{ICI}$ recipients compared to the platinum-based chemotherapy recipients (HR: $0.60,95 \% \mathrm{Cl}$
0.44 to $0.81, \mathrm{P}<0.0009, \mathrm{I}=0 \%, 3 \mathrm{RCTs}, 367$ participants) Analysis 1.10 .

ECOG PS 1 and PD-L1 expression $\geq \mathbf{5 0} \%$. There was evidence of a difference in OS favouring people who received single-agent $\mathrm{ICI}$ compared to platinum-based chemotherapy recipients (HR: 0.67, $95 \% \mathrm{Cl} 0.55$ to $0.82, \mathrm{P}=0.0001, \mathrm{I}^{2}=0 \%, 3 \mathrm{RCTs}, 741$ participants), Analysis 1.10.

The pooled results showed a difference in OS in favour of people receiving single-agent $\mathrm{ICl}$ compared to platinum-based chemotherapy (HR: $0.65,95 \% \mathrm{Cl} 0.55$ to $0.77, \mathrm{P}<0.00001, \mathrm{I}=0 \%$, 3 RCTs, 1108 participants) Analysis 1.10.There was no evidence of a difference between the two subgroups, ECOG PS 0 and 1, (Test for subgroup differences: $\left.\mathrm{Chi}^{2}=0.40, \mathrm{df}=1(\mathrm{P}=0.52), \mathrm{I}^{2}=0 \%\right)$ Analysis 1.10 .

\section{- $x$. OS by histology and expression $\geq 1 \%$}

Two trials (Carbone 2017; Mok 2019) provided data for this outcome.

Squamous and PD-L1 expression $\geq \mathbf{1} \%$. There was evidence of a difference in OS favouring the participants who received singleagent $\mathrm{ICl}$ when compared to platinum-based chemotherapy (HR: $0.76,95 \% \mathrm{Cl} 0.63$ to $0.93, \mathrm{P}=0.006, \mathrm{I}=0 \%, 2 \mathrm{RCTs}, 621$ participants) Analysis 1.11.

Non-squamous and PD-L1 expression $\geq 1 \%$. There was no evidence of a difference between the two groups, single-agent $\mathrm{ICl}$ and standard chemotherapy, (HR: $0.99,95 \% \mathrm{Cl} 0.73$ to $1.33, \mathrm{P}=0.94$, $\mathrm{I} 2=72 \%, 2 \mathrm{RCTs}, 1194$ participants). Analysis 1.11 .

The pooled results showed no difference in OS between singleagent $\mathrm{ICl}$ and standard chemotherapy, (HR: $0.89,95 \% \mathrm{Cl} 0.73$ to $1.07, \mathrm{P}=0.20, \mathrm{I}^{2}=56 \% 2 \mathrm{RCTs}, 1815$ participants) Analysis 1.11 . However, when applying a fixed-effect model we found evidence of a difference between the two groups and the level of heterogeneity remained unchanged (HR: $0.88,95 \% \mathrm{Cl} 0.78$ to $0.98, \mathrm{P}=0.03, \mathrm{I}=56 \%$ 2 RCTs, 1815 participants) (analysis not shown).

In addition, there was no evidence of any differences according to histology subgroups (squamous versus non-squamous) either applying a random-effects model (Test for subgroup differences: $\left.\mathrm{Chi}^{2}=2.00, \mathrm{df}=1(\mathrm{P}=0.16), \mathrm{I}^{2}=50.0 \%\right)$ Analysis 1.11 or a fixed-effect model (Test for subgroup differences: $\mathrm{Chi}^{2}=3.09, \mathrm{df}=1(\mathrm{P}=0.08)$, $\left.I^{2}=67.7 \%\right)$ (analysis not shown).

\section{- xi. OS by histology and expression $\geq 50 \%$}

Three trials provided data for this outcome ( Herbst 2020; Mok 2019 ; Reck 2016).

Squamous and PD-L1 expression $\geq \mathbf{5 0} \%$. There was evidence of a difference in OS among people receiving single-agent $\mathrm{ICl}$ compared to participants treated with standard chemotherapy (HR: 0.57, 95\% $\mathrm{Cl} 0.43$ to $0.76, \mathrm{P}=0.0001, \mathrm{I}=0 \%, 3 \mathrm{RCTs}, 327$ participants) Analysis 1.12

Non-squamous and PD-L1 expression $\geq \mathbf{5 0 \%}$. Three trials ( Herbst 2020; (Mok 2019; Reck 2016) provided data for this subgroup. There was evidence of a difference in OS favouring the group who received single-agent $\mathrm{ICl}$ compared to standard chemotherapy (HR:0.69, 
$95 \% \mathrm{Cl} 0.55$ to $0.87, \mathrm{P}=0.002, \mathrm{I}=26 \%, 3 \mathrm{RCTs}, 782$ participants) Analysis 1.12

The pooled results showed a difference in OS between single-agent $\mathrm{ICl}$ and standard platinum-based chemotherapy regardless of histology subtype in people with PD-L1 expression $\geq 50 \%$ (HR: 0.66, $95 \% \mathrm{Cl} 0.56$ to $0.77, \mathrm{P}<0.00001, \mathrm{I}^{2}=0 \%, 3 \mathrm{RCTs}, 1109$ participants) Analysis 1.12. There was no evidence of a difference between the two histology subtypes (squamous and non-squamous) (Test for subgroup differences: $\mathrm{Chi}^{2}=1.08, \mathrm{df}=1, \mathrm{P}=0.30, \mathrm{I}^{2}=7.6 \%$ ) Analysis 1.12 .

\section{Primary outcome - Progression-free survival (PFS)}

\section{- a. Main analysis: PFS by PD-L1 expression}

Five trials provided data for this outcome (Carbone 2017; Herbst 2020; Mok 2019; Reck 2016; Sezer 2020).

PD-L1 expression <1\% (negative PD-L1). None of the included trials reported PFS data by this subgroup.

PD-L1 expression $\geq 1 \%$ (positive PD-L1). Three trials (Carbone 2017; Herbst 2020; Mok 2019) provided data for this subgroup. There was no evidence of a difference in PFS between the group who received single-agent $\mathrm{ICl}$ or platinum-based chemotherapy (HR: $0.99,95 \% \mathrm{Cl} 0.79$ to $1.24, \mathrm{P}=0.95, \mathrm{I}^{2}=80 \% 3 \mathrm{RCTs}, 2369$ participants) Analysis 1.13.

PD-L1 expression $\mathbf{2} \mathbf{5 0} \%$ (high PD-L1). Five trials (Carbone 2017; Herbst 2020; Mok 2019; Reck 2016; Sezer 2020) reported PFS results. There may be evidence of a difference in PFS favouring the group who received single-agent $\mathrm{ICl}$ compared to platinum-based chemotherapy (HR: $0.68,95 \% \mathrm{Cl} 0.52$ to $0.88, \mathrm{P}=0.003 \mathrm{I}^{2}=80 \%$, 5 RCTs, 1886 participants; low certainty of evidence) Analysis 1.13 . The certainty for this outcome was downgraded one level due to study limitations, and one level due to inconsistency.

The pooled results showed a difference in PFS favouring the singleagent $\mathrm{ICl}$ compared to the platinum-based chemotherapy, (HR: $0.79,95 \% \mathrm{Cl} 0.63$ to $0.99 \mathrm{P}=0.04 \mathrm{I}^{2}=79.2 \%, 5 \mathrm{RCTs}, 4255$ participants) Analysis 1.13 . There was evidence of a difference between PD-L1 $\geq 1 \%$ and $\geq 50 \%$ subgroups (Test for subgroup differences: $\mathrm{Chi}^{2}=4.82, \mathrm{df}=1, \mathrm{P}=0.03, \mathrm{I}^{2}=79.2 \%$ ) Analysis 1.13.

\section{- b. Subgroup analyses:}

\section{- i. PFS by Tumour Mutaution Burden (TMB)}

Four trials (Carbone 2017; Herbst 2020; Mok 2019; Rizvi 2020) provided data for this outcome.

TMB - High. There was evidence of a difference in PFS favouring participants who received single-agent $\mathrm{ICl}$ compared to platinumbased chemotherapy (HR: $0.72,95 \% \mathrm{Cl} 0.60$ to $0.86, \mathrm{P}=0.0003, \mathrm{I}^{2}=$ 0\%, 4 RCTs, 655 participants) Analysis 1.14

TMB - Low. There was no evidence of a difference in PFS for people who received single-agent $\mathrm{ICl}$ compared to platinumbased chemotherapy (HR: $1.24,95 \% \mathrm{Cl} 1.00$ to $1.55, \mathrm{P}=0.05, \mathrm{l}=$ 69\%, 4 RCTs, 1380 participants) Analysis 1.14. In contrast, when applying a fixed-effect model there was a difference in favour of the chemotherapy group (HR: $1.22,95 \% \mathrm{Cl} 1.08$ to $1.37, \mathrm{P}=0.001, \mathrm{I}^{2}=$ 69\%, 4 RCTs, 1380 participants) (analysis not shown).
The pooled results showed no difference between people who received single-agent $\mathrm{ICl}$ compared to those who received platinum-based chemotherapy (HR: $0.97,95 \% \mathrm{Cl} 0.76$ to $1.22, \mathrm{P}=$ $0.77, \mathrm{I}^{2}=80 \%, 4 \mathrm{RCTs}, 2035$ participants) Analysis 1.14. The pooled results and the level of heterogeneity did not change with fixedeffect model (HR: $1.03,95 \% \mathrm{Cl} 0.93$ to $1.14, \mathrm{P}=0.54, \mathrm{~L} 2=80 \%, 4$ RCTs, 2035 participants) (analysis not shown). There was evidence of a difference between TMB-high and TMB-low subgroups either when applying a random-effects model (Test for subgroup differences: $\left.\mathrm{Chi}^{2}=14.47, \mathrm{df}=1(\mathrm{P}=0.0001), \mathrm{I}^{2}=93.1 \%\right)$ Analysis 1.14 or a fixedeffect model (Test for subgroup differences: $\mathrm{Chi}^{2}=23.29$, df $=1(\mathrm{P}<$ $0.00001), I^{2}=95.7 \%$ ) (analysis not shown).

\section{$\underline{\text { 3. Secondary outcomes - Overall response rate (ORR) }}$}

\section{- a. Main analysis: ORR by PD-L1 expression}

Three trials (Herbst 2020; Mok 2019; Reck 2016;Sezer 2020) provided data for this outcome.

PD-L1 expression <1\% (negative PD-L1).: None of the included trials reported ORR for this subgroup.

PD-L1 expression $\geq 1 \%$ (positive PD-L1). Two trials (Herbst 2020; Mok 2019) reported ORR showing no evidence of a difference in the group who received single-agent $\mathrm{ICl}$ compared to standard platinum-based chemotherapy (RR: $0.99,95 \% \mathrm{Cl} 0.86$ to $1.15, \mathrm{P}=$ $0.90, \mathrm{I}^{2}=0 \%, 2$ RCTs, 1828 participants) Analysis 1.15.

PD-L1 expression $\mathbf{2} \mathbf{5 0} \%$ (high PD-L1). Four trials (Herbst 2020; Mok 2019; Reck 2016; Sezer 2020 ) reported ORR. There may be evidence of a difference in favour of single-agent $\mathrm{ICl}$ compared with platinum-based chemotherapy (RR: $1.40,95 \% \mathrm{Cl} 1.12$ to $1.75, \mathrm{P}=$ 0.003 , I 2=60\%, 4 RCTs, 1672 participants, low-certainty evidence) Analysis 1.15. The certainty for this outcomes was downgraded one level due to study limitations, and one level due to inconsistency.

There was evidence of a difference between the PD-L1 $\geq 1 \%$ and PD$\mathrm{L} 1 \geq 50 \%$ subgroups (Test for subgroup differences: $\mathrm{Chi}^{2}=6.50, \mathrm{df}=$ $\left.1(\mathrm{P}=0.01), \mathrm{I}^{2}=84.6 \%\right)$ Analysis 1.15 .

- b. Subgroup analyses

- i. ORR by Tumour Mutational Burden (TMB)

Three trials (Carbone 2017; Mok 2019, Rizvi 2020) provided data for this outcome.

TMB - High. There was no evidence of a difference in ORR in the group of people who received single-agent $\mathrm{ICl}$ compared to platinum-based chemotherapy was reported (RR: $1.25,95 \% \mathrm{Cl} 0.99$ to $1.59, \mathrm{P}=0.06, \mathrm{I}=1 \%$, 3 RCTs, 599 participants) Analysis 1.16 .

TMB - Low. There was evidence of a difference in ORR favouring platinum-based chemotherapy group (RR: $0.73,95 \% \mathrm{Cl} 0.59$ to 0.91 , $\mathrm{P}=0.004 . \mathrm{I}^{2}=0 \%, 3 \mathrm{RCTs}, 1047$ participants) Analysis 1.16 .

The pooled results showed no difference in ORR between the two treatment groups (RR: $0.97,95 \% \mathrm{Cl}: 0.73$ to $1.27, \mathrm{P}=0.80, \mathrm{I}^{2}=64 \%$, 1646 participants) Analysis 1.16. There was evidence of a difference between the two subgroups (TMB-High and TMB-Low) (Test for subgroup differences: $\left.\mathrm{Chi}^{2}=10.80, \mathrm{df}=1(\mathrm{P}=0.001), \mathrm{I}^{2}=90.7 \%\right)$.

\section{Secondary outcome - Adverse events (AEs)}


- a. Main analysis - AEs overall

None of the included trials provided data by PD-L1 expression for AEs.

Five included trials (Carbone 2017; Herbst 2020; Mok 2019; Reck 2016; Rizvi 2020) reported grade three to five AEs. For overall population and not according to PD-L1 expression levels.
Overall, there was evidence of reduced grade 3 to 5 adverse events in people who received single-agent $\mathrm{ICl}$ compared with platinumbased chemotherapy (RR: $0.43,95 \% \mathrm{Cl} 0.36$ to $0.52, \mathrm{P}<0.00001$, $\mathrm{I}^{2}=41 \%, 5 \mathrm{RCTs}, 6692$ participants, low-certainty evidence) Analysis 1.17 ; Figure 6.

Figure 6. Forest plot of comparison: Single Immuno versus Chemotherapy, outcome: 4.7 Adverse Events grade 3-5.

\begin{tabular}{|c|c|c|c|c|c|}
\hline \multirow[b]{2}{*}{ Study or Subgroup } & \multicolumn{2}{|c|}{ Immunotherapy } & \multicolumn{2}{|c|}{ Chemotherapy } & \multirow[b]{2}{*}{ Weight } \\
\hline & Events & Total & Events & Total & \\
\hline \multicolumn{6}{|c|}{ 1.17.1 Adverse Events grade 3-4 } \\
\hline Carbone 2017 & 47 & 267 & 133 & 263 & $17.9 \%$ \\
\hline Herbst 2020 & 37 & 277 & 116 & 263 & $15.7 \%$ \\
\hline Mok 2019 & 100 & 636 & 238 & 615 & $22.5 \%$ \\
\hline Reck 2016 & 46 & 154 & 77 & 150 & $17.8 \%$ \\
\hline Rizvi 2020 & 53 & 369 & 116 & 352 & $17.6 \%$ \\
\hline Subtotal $(95 \%$ CI) & & 1703 & & 1643 & $91.4 \%$ \\
\hline Total events: & 283 & & 680 & & \\
\hline \multicolumn{6}{|c|}{ Heterogeneity: $\mathrm{Tau}^{2}=0.03 ; \mathrm{Chi}^{2}=10.46, \mathrm{df}=4(\mathrm{P}=0.03) ; \mathrm{I}^{2}=62 \%$} \\
\hline \multicolumn{6}{|c|}{ Test for overall effect: $\mathrm{Z}=8.84(\mathrm{P}<0.00001)$} \\
\hline \multicolumn{6}{|c|}{ 1.17.2 Adverse Events grade 5 (toxic deaths) } \\
\hline Carbone 2017 & 2 & 267 & 3 & 263 & $1.0 \%$ \\
\hline Herbst 2020 & 0 & 277 & 1 & 263 & $0.3 \%$ \\
\hline Mok 2019 & 13 & 636 & 14 & 615 & $5.1 \%$ \\
\hline Reck 2016 & 2 & 154 & 3 & 150 & $1.1 \%$ \\
\hline Rizvi 2020 & 2 & 369 & 3 & 352 & $1.0 \%$ \\
\hline Subtotal (95\% CI) & & 1703 & & 1643 & $8.6 \%$ \\
\hline Total events: & 19 & & 24 & & \\
\hline
\end{tabular}

Heterogeneity: $\mathrm{Tau}^{2}=0.00 ; \mathrm{Chi}^{2}=0.57, \mathrm{df}=4(\mathrm{P}=0.97) ; \mathrm{I}^{2}=0 \%$

Test for overall effect: $\mathrm{Z}=0.83(\mathrm{P}=0.40)$

$\begin{array}{llllll}\text { Total }(\mathbf{9 5 \%} \text { CI }) & & \mathbf{3 4 0 6} & & \mathbf{3 2 8 6} & \mathbf{1 0 0 . 0 \%} \\ \text { Total events: } & 302 & & 704 & & \end{array}$

Heterogeneity: $\mathrm{Tau}^{2}=0.03 ; \mathrm{Chi}^{2}=15.27, \mathrm{df}=9(\mathrm{P}=0.08) ; \mathrm{I}^{2}=41 \%$

Test for overall effect: $Z=8.93(\mathrm{P}<0.00001)$

Test for subgroup differences: $\mathrm{Chi}^{2}=4.08, \mathrm{df}=1(\mathrm{P}=0.04), \mathrm{I}^{2}=75.5 \%$

Adverse events grade 3 to 4 . There was may be evidence of a difference with reduced adverse events grade 3 to 4 in favour of single-agent ICls compared to platinum-based chemotherapy (RR: $0.41,95 \% \mathrm{Cl} 0.33$ to $0.50, \mathrm{P}<0.00001, \mathrm{l}=62 \%, 5$ RCTs, 3346 participants, low-certainty evidence) Analysis 1.17 ; Figure 6. The certainty for this outcomes was downgraded one level due to study limitations, and one level due to inconsistency.

Removing data from Reck 2016, reduced the heterogeneity level to $13 \%$ without changing the results (RR: $0.38,95 \% \mathrm{Cl} 0.33$ to 0.44 , $\mathrm{P}<0.00001, \mathrm{I}^{2}=13 \%, 4 \mathrm{RCTs}, 3042$ participants) (analysis not shown).

Adverse events grade five (toxic deaths). There was no evidence of a difference in toxic deaths (grade 5 AEs) in participants who received single-agent $\mathrm{ICl}$ compared to platinum-based chemotherapy (RR: $0.78,95 \% \mathrm{Cl} 0.43$ to $1.41, \mathrm{P}=0.40, \mathrm{I}^{2}=0 \%, 5$ RCTs, 3346 participants) Analysis 1.17 ; Figure 6.

In addition, there was evidence of subgroup differences between grade 3 to 4 and grade 5 adverse events (Test for subgroup differences: $\left.\mathrm{Chi}^{2}=4.08, \mathrm{df}=1(\mathrm{P}=0.04), \mathrm{I}^{2}=75.5 \%\right)$.
Risk Ratio

Risk Ratio

M-H, Random, $95 \%$ CI

Single or combined immune checkpoint inhibitors compared to first-line platinum-based chemotherapy with or without bevacizumab for
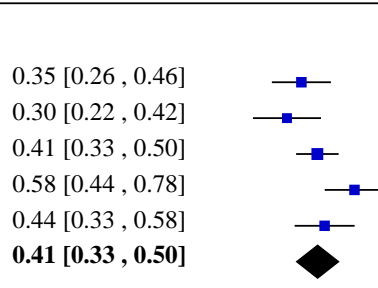

$0.66[0.11,3.90]$ $0.32[0.01,7.74]$ $0.90[0.43,1.89]$ $0.65[0.11,3.83]$ $0.64[0.11,3.78]$ $0.78[0.43,1.41]$

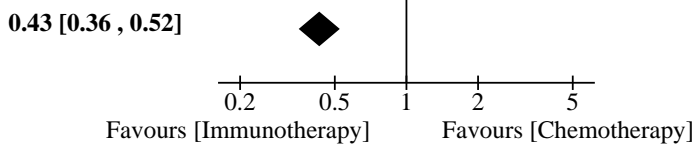

5. Secondary outcome - Health-related quality of life (HRQoL)

HRQoL was measured in only one multicentre open-label phase 3 trial (Reck 2016) comparing pembrolizumab to platinum-based chemotherapy in advanced NSCLC.The quality of life was measured using the European Organisation for Research and Treatment of Cancer (EORCT) Quality of Life Questionnaire Core 30 items (QOLC30); and the EORTC quality of life questionnaire lung cancer 13 items (QLQ-LC13) and the European Quality of Life 5 dimensions-3 Level (EQ-5D-3L). The QOL-C30 measures five functional demotions namely: physical, role, cognitive and social and three symptoms items fatigue, fatigue, nausea or vomiting and pain, six single items (dyspnoea, sleep disturbance, appetite loss, constipation, diarrhoea and financial impact. and a global health and quality of life scale (GHS/QOL) Osoba 1998.

The three instruments were administrated on day one of cycle one to three and every nine weeks thereafter, at the treatment discontinuation and at the 30 days safety checks. The compliance with the questionnaires was more than $90 \%$ at baseline and around $80 \%$ at week 15 for both groups. 
Overall HRQOL using QOL-C30 GHS/QOL. At week 15, there was evidence of improvement from baseline in favour of people who received pembrolizumab compared with platinum-based chemotherapy group, the difference was 7.8 points ( MD: $7.80,95 \%$ $\mathrm{Cl} 2.45$ to 13.15 , $1 \mathrm{RCT}, 297$ participants) Analysis 1.18.

\section{Time to symptoms deterioration using quality of life questionnaire lung cancer 13 items (QLQ-LC13)}

Time to deterioration defined as the time to the first onset of 10 points or more decrease from baseline of the following symptoms: cough, chest pain or dyspnoea. There was evidence of a difference in time to deterioration of the composite symptoms favouring the pembrolizumab group (HR:0.66, 95\% Cl 0.440 to 99, $1 \mathrm{RCT}, 194$ participants) Analysis 1.19.

\section{Improvement on Quality of Life Questionnaire Core 30 items (QOL-C30)}

The proportion of individuals who improved on the global health score and quality of life (GHS/QOL), functional and symptoms scales on the QLQ-C30 were reported at week 15. Improvement was defined as a 10-point greater increase in functional scores or a 10point greater decrease in symptoms scores.

At week 15, a greater improvement in the GHS/QoL score from baseline was may be evident among pembrolizumab group (RR:1.51, 95\% Cl 1.08 to 2.10, 1 RCT, 297 participants, lowcertainty evidence) Analysis 1.22. The certainty for this outcomes was downgraded one level due to study limitations, and one level due to imprecision. Similarly, fewer individuals treated with pembrolizumab had deteriorated fatigue (RR:1.61, 95\% Cl 1.21 to 2.13, 1 RCT, 297 participants) and pain (RR $1.39,95 \% \mathrm{Cl} 1.05$ to 1.84 , RCT, 297 participants), respectively Analysis 1.22. However, there was no evidence of a difference between the two groups in physical function (RR:1.09, 95\% Cl 0.74 to $1.61,1 \mathrm{RCT}, 297$ participants), role function (RR:1.29, 95\% Cl 0.92, 1.81, $1 \mathrm{RCT}, 297$ participant), emotional (RR $0.81,95 \% \mathrm{Cl} 0.60$ to $1.10,1 \mathrm{RCT}, 297$ participant), cognitive (RR $0.75,95 \% \mathrm{Cl} 0.50$ to $1.15,1 \mathrm{RCT}, 297$ participants) and social functions (RR 1.20, 95\% Cl 0.85 to $1.69,1 \mathrm{RCT}, 297$ participant) Analysis 1.22. There was no evidence of a difference between the group in nausea and vomiting (RR $0.90,95 \% \mathrm{Cl} 0.55$ to $1.50,1 \mathrm{RCT}$, 297 participants), dyspnoea (RR $1.24,95 \% \mathrm{Cl} 0.88$ to $1.73,1 \mathrm{RCT}, 297$ participants), sleep disturbance (RR $0.89,95 \% \mathrm{Cl} 0.66$ to $1.21,1 \mathrm{RCT}$, 297 participants), appetite loss (RR $1.12,95 \% \mathrm{Cl} 0.82$ to $1.54,1 \mathrm{RCT}$, 297 participants) and constipation (RR $1.33,95 \% \mathrm{Cl} 0.84$ to $2.12,1$ RCT, 297 participants) Analysis 1.22.

There was evidence of a difference of the proportion of people who reported improved diarrhoea favouring chemotherapy (RR 0.49 , $95 \% \mathrm{Cl} 0.25$ to $0.94,1 \mathrm{RCT}, 297$ participants) Analysis 1.22.

The number of individuals who reported any improvement in their financial difficulties at week 15 did not differ between the groups (RR 0.88, 95\% Cl 0.54 to 1.42, RCT, 297 participants, Analysis 1.22 .

\section{Deterioration on Quality of Life Questionnaire Core $\mathbf{3 0}$ items (QOL-C30)}

The proportion of individuals who deteriorated on the global health score and quality of life (GHS/QOL), functional and symptoms scales on the QLQ-C30 were reported at week 15. Deterioration was defined as a 10-point greater decrease in functional scores or a 10point greater increase in symptoms scores.
At week 15, the deterioration in GHS/QoL scores were similar between the two groups (RR $0.71,95 \% \mathrm{Cl} 0.48$ to $1.06,1 \mathrm{RCT}, 297$ participants) Analysis 1.21.

Fewer participants in the pembrolizumab group had deteriorated status on physical functioning (RR $0.55,95 \% \mathrm{Cl} 0.38$ to $0.82,1 \mathrm{RCT}$, 297 participants), role functioning (RR $0.61,95 \% \mathrm{Cl} 0.42$ to $0.88,1$ RCT, 297 participants) and social functioning scores (RR 0.61, 95\% Cl 0.41 to $0.89,1 \mathrm{RCT}, 297$ participants) Analysis 1.21. However, there was no evidence of a difference in emotional (RR 1.20, 95\% $\mathrm{Cl} 0.67$ to $2.14,1 \mathrm{RCT}, 297$ participants) and cognitive functioning scores (RR 1.03, 95\% Cl 0.70 to 1.51,1 RCT, 297 participants) Analysis 1.21. Among the pembrolizumab group, fewer individuals reported deterioration their in fatigue (RR $0.43,95 \% \mathrm{Cl} 0.29$ to $0.63,1 \mathrm{RCT}$, 297 participants), nausea and vomiting (RR $0.48,95 \% \mathrm{Cl} 0.28$ to $0.81,1 \mathrm{RCT}, 297$ participants), dyspnoea (RR $0.43,95 \% \mathrm{Cl} 0.27$ to $0.68,1 \mathrm{RCT}, 297$ participants), appetite loss (RR 0.59, 95\% Cl 0.37 to $0.94,1 \mathrm{RCT}, 297$ participants) and constipation symptoms (RR $0.63,95 \% \mathrm{Cl} 0.41$ to $0.97,1 \mathrm{RCT}, 297$ participants) Analysis 1.21. There was no evidence of a difference between the number of individuals in the pembrolizumab and chemotherapy groups who reported a worsening in their pain (RR $0.75,95 \% \mathrm{Cl} 0.47$ to $1.18,1$ RCT, 297 participants,) and diarrhoea symptoms (RR $0.73,95 \% \mathrm{Cl} 0.44$ to $1.23,1 \mathrm{RCT}, 297$ participants) Analysis 1.21 . The number of individuals who reported a deterioration in their financial difficulties did not differ between the groups (RR 0.75, 95\% $\mathrm{Cl} 0.49$ to $1.15,1 \mathrm{RCT}, 297$ participants) Analysis 1.21.

\section{Stable status on Quality of Life Questionnaire Core 30 items (QLQ-C30)}

The proportion of individuals who reported no changes or stable status on the global health score and quality of life (GHS/QOL), functional and symptoms scales on the QLQ-C30 was reported at week 15 in Reck 2016. On the GHS/QOL scores the number of individuals who reported "stable status" did not differ between the two groups (RR 0.89, 95\% Cl 0.68 to $1.17,1 \mathrm{RCT}, 297$ participants) Analysis 1.20. In contrast, more individuals in the pembrolizumab reported their physical function was stable at week 15 (RR 1.35, $95 \% \mathrm{Cl} 1.05$ to $1.74,1 \mathrm{RCT}, 297$ participants). However, there was no evidence of a difference between the two groups regarding number of individuals who reported " stable status" on role (RR 1.16, 95\% $\mathrm{Cl} 0.88$ to $1.55,1 \mathrm{RCT}, 297$ participants), emotional (RR $1.11,95 \% \mathrm{Cl}$ 0.88 to $1.39,1 \mathrm{RCT}, 297$ participants), cognitive (RR 1.12, 95\% Cl 0.89 to $1.40,1 \mathrm{RCT}, 297$ participants) and social functions (RR 1.21, 95\% $\mathrm{Cl} 0.92$ to 1.58, 1 RCT, 297 participants) Analysis 1.20.

There was evidence of a difference favouring pembrolizumab group in the proportion of individuals who reported "stable status" of nausea and vomiting (RR $1.24,95 \% \mathrm{Cl} 1.05$ to $1.47,1 \mathrm{RCT}, 297$ participants), insomnia (RR $1.36,95 \% \mathrm{Cl} 1.03$ to $1.79,1 \mathrm{RCT}$, 297 participants,) and diarrhoea symptoms (RR 1.21, 95\% Cl 1.04 to $1.40,1 \mathrm{RCT}, 297$ participants) Analysis 1.20. However, the two groups did not differ regarding number of individuals who reported "stable status" on fatigue (RR $1.19,95 \% \mathrm{Cl} 0.82$ to $1.71,1 \mathrm{RCT}$, 297 participants), pain (RR $0.82,95 \% \mathrm{Cl} 0.62$ to $1.10,1 \mathrm{RCT}, 297$ participants), dyspnoea (RR $1.28,95 \% \mathrm{Cl} 0.99$ to $1.64,1 \mathrm{RCT}, 297$ participants), appetite loss (RR 1.16, 95\% Cl 0.90 to 1.49, 1 RCT, 297 participants), and constipation symptoms (RR $1.09,95 \% \mathrm{Cl} 0.89$ to 1.33, 1 RCT, 297 participants) Analysis 1.20.

There was no significant differences between pembrolizumab and platinum-based chemotherapy in the number of people who

Single or combined immune checkpoint inhibitors compared to first-line platinum-based chemotherapy with or without bevacizumab for 24 people with advanced non-small cell lung cancer (Review)

Copyright (c) 2020 The Cochrane Collaboration. Published by John Wiley \& Sons, Ltd. 
were stable financially (RR 1.16, 95\% Cl 0.96 to 1.41, RCT, 297 participants) Analysis 1.20

\section{Comparison 2: First-line combined ICI versus platinum-based chemotherapy}

1. Primary outcome - Overall survival (OS)

- a. Main analysis: . OS and PDL-1 expressions

Two trials (Hellmann 2018; Rizvi 2020) comparing double ICI (nivolumab plus ipilimumab or durvalumab plus tremelimumab) versus standard platinum-based chemotherapy reported results. The OS data were reported according to the following PD-L1 expression categories.

PD-L1 expression $<1 \%$ (negative PD-L1). There was evidence of a difference in OS favouring people who received the combination of ICl compared to platinum-based chemotherapy (HR: $0.67,95 \% \mathrm{Cl}$ 0.55 to $0.81, \mathrm{P}<0.0001 \mathrm{I}^{2}=0 \%, 2 \mathrm{RCTs}, 532$ participants) Analysis 2.1 ; Figure 7.

Figure 7. Forest plot of comparison: Combined Immuno versus Chemotherapy, outcome: 5.1 Overall Survival by PDL1 expression.

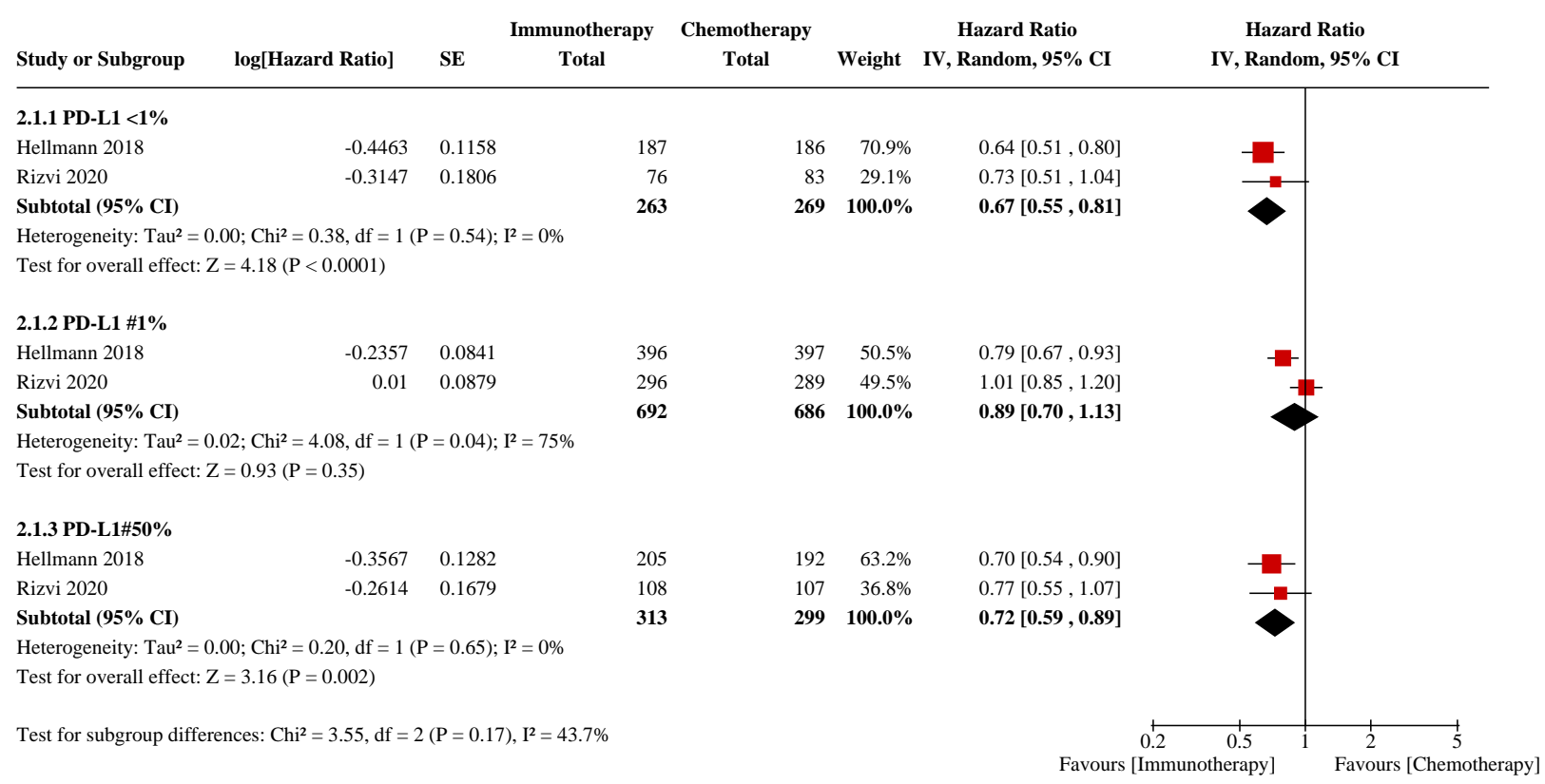

PD-L1 expression $\geq 1 \%$ (positive PD-L1). There was no evidence of a difference in $\mathrm{OS}$ between double $\mathrm{ICl}$ and platinum-based chemotherapy (HR: $0.89,95 \% \mathrm{Cl} 0.70$ to $1.13 ; \mathrm{P}=0.35, \mathrm{I}^{2}=75 \%$, 2RCTs, participants $=1378$ ) Analysis 2.1; Figure 7.

PD-L1 expression $\geq \mathbf{5 0 \%}$ (high PD-L1).There was probably evidence of a difference in favour of participants who received combination of $\mathrm{ICl}$ compared to people treated with platinumbased chemotherapy (HR: $0.72,95 \% \mathrm{Cl} 0.59$ to $0.89, \mathrm{P}=0.002, \mathrm{I}=$ $0 \%, 2$ RCTs, 612 participants, moderate-certainty evidence) Analysis 2.1 ; Figure 7. The certainty for this outcomes was downgraded one level due to study limitations.

Although there was no difference according to PD-L1 expression by random-effects model (Test for subgroup differences: $\mathrm{Chi}^{2}=3.55$,

$\left.\mathrm{df}=2(\mathrm{P}=0.17), \mathrm{I}^{2}=43.7 \%\right)$ Analysis 2.1 , Figure 7 , evidence of a subgroup difference was observed by fixed-effect model (Test for subgroup differences: $\mathrm{Chi}^{2}=7.51, \mathrm{df}=2(\mathrm{P}=0.02), \mathrm{I}^{2}=73.4 \%$

- b. Subgroup analyses

\section{- i. OS and Tumour Mutational Burden (TMB)}

TMB was assessed on tissue in Hellmann 2018 and on plasma ctDNA in Rizvi 2020. Thresholds to define high TMB were $\geq 10$ mut/Mb (Hellmann 2018) and $\geq 20$ Mut/Mb (Rizvi 2020), respectively.

TMB - Low. There was no evidence of a difference in OS between double $\mathrm{ICl}$ and platinum-based chemotherapy groups (HR: 0.93, $95 \% \mathrm{Cl} 0.61$ to $1.43, \mathrm{P}=0.75, \mathrm{I}^{2}=86 \%, 2 \mathrm{RCTs}, 769$ participants) Analysis 2.2 ; Figure 8. 
Figure 8. Forest plot of comparison: Combined Immuno versus Chemotherapy, outcome: 5.2 Overall Survival by Tumor Mutational Burden.

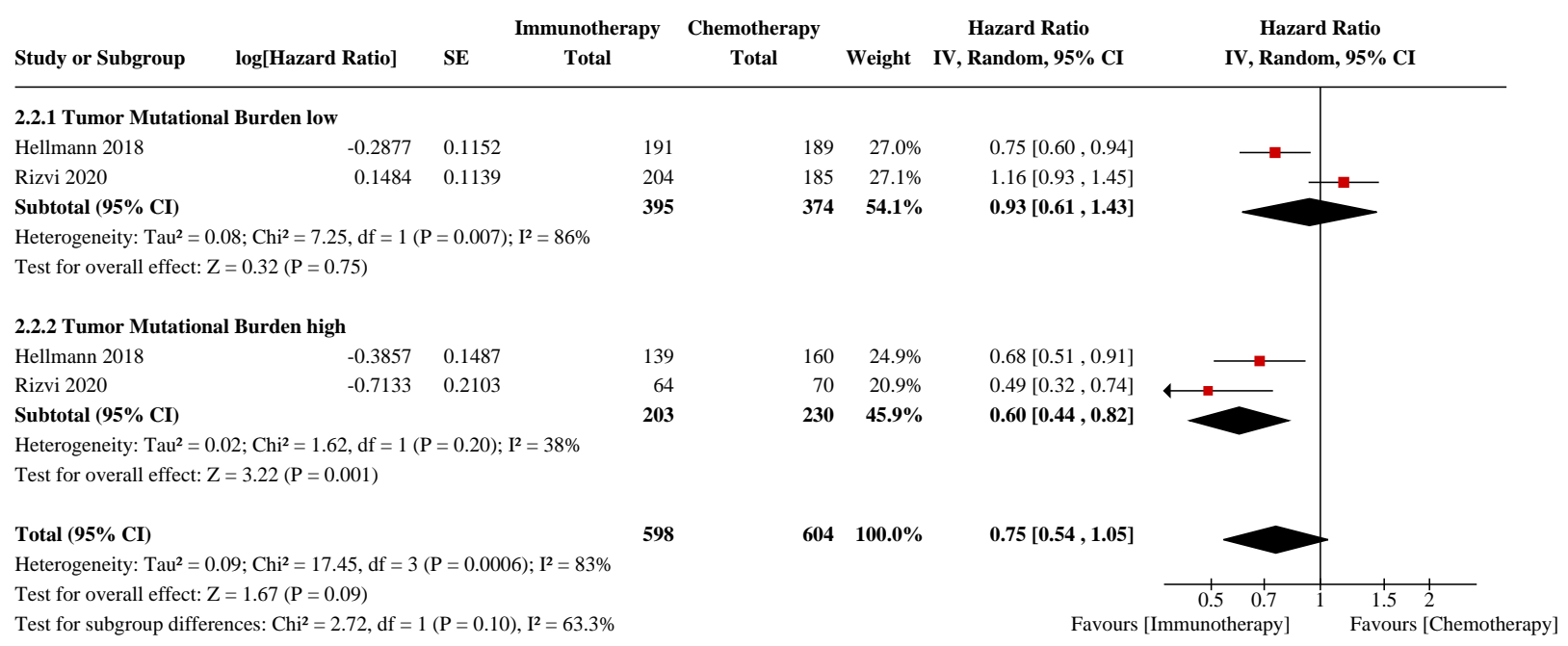

TMB - High. There was evidence of a difference in OS favouring people who were treated with combined ICl compared to platinumbased chemotherapy (HR: $0.60,95 \% \mathrm{Cl} 0.44$ to $0.82, \mathrm{P}=0.001, \mathrm{I}=$ $38 \%, 2$ RCTs, 433 participants) Analysis 2.2, Figure 8.

The pooled results showed no difference between double $\mathrm{ICI}$ and platinum-based chemotherapy (HR: $0.75,95 \% \mathrm{Cl} 0.54$ to $1.05, \mathrm{P}=$ $0.09,12=83 \%, 2$ RCTs, 1202 participants) Analysis 2.2, Figure 8. When applying a fixed-effect model there was evidence of a difference between treatments (HR: $0.82,95 \% \mathrm{Cl} 0.72$ to $0.94, \mathrm{P}=0.003, \mathrm{I}=$ $83 \%, 2$ RCTs, 1202 participants) (analysis not shown).

There was no evidence of a difference between the two TMB subgroups (Test for subgroup differences: $\mathrm{Chi}^{2}=2.72, \mathrm{df}=1(\mathrm{P}$ $=0.10), I^{2}=63.3 \%$ ) Analysis 2.2, Figure 8 when using a randomeffects model. When applying a fixed-effect model there was a difference between the TMB-High and the TMB-low subgroups (Test for subgroup differences: $\left.\mathrm{Chi}^{2}=8.59, \mathrm{df}=1(\mathrm{P}=0.03), \mathrm{I}^{2}=88.4 \%\right)$ (analysis not shown).

- ii. OS by age and PD-L1 expression $\geq 1 \%$ and $P D-L 1$ expression $>50 \%$

None of the included trials reported this outcome.

- iii. OS by gender and PD-L1 expression $\geq 1 \%$ and PD-L1 expression $>50 \%$

None of the included trials reported this outcome.

- iv. OS by smoking status and PD-L1 expression $\geq 1 \%$ and $P D-L 1$ expression $>50 \%$

None of the included trials reported this outcome.

- v. OS by ECOG PS and PD-L1 expression $\geq 1 \%$. and $P D-L 1$ expression $>50 \%$

None of the included trials reported this outcome. .

- vi. OS by histology and PD-L1 expression $\geq 1 \%$.and PD-L1 expression $>50 \%$

Single or combined immune checkpoint inhibitors compared to first-line platinum-based chemotherapy with or without bevacizumab for people with advanced non-small cell lung cancer (Review)

Copyright (c) 2020 The Cochrane Collaboration. Published by John Wiley \& Sons, Ltd.
None of the included trials reported this outcome.

\section{Primary outcome - Progression-free survival (PFS)}

- a. Main analysis: PFS by PD-L1 expression

None of the included trials reported this outcome.

- b. Subgroup analyses:

- i. Progression-free survival (PFS) by Tumour Mutational Burden (TMB)

Two trials Hellmann 2018 and Rizvi 2020 reported PFS results by TMB.

TMB - Low. There was no evidence of a difference on PFS outcome between double $\mathrm{ICl}$ and platinum-based chemotherapy (HR: $1.29,95 \% \mathrm{Cl} 0.90$ to $1.85, \mathrm{P}=0.17, \mathrm{I}^{2}=80 \%, 2$ RCTs, 769 participants) Analysis 2.3. When applying the fixed-effect model, results changed with evidence of a difference in favour of platinumbased chemotherapy and no changes in the heterogeneity level (HR: $1.30,95 \% \mathrm{Cl} 1.10$ to $1.52, \mathrm{P}=0.002, \mathrm{I}^{2}=80 \%, 2$ RCTs, 769 participants) (analysis not shown).

TMB - High. There was evidence of a difference in PFS among people who were treated with double $\mathrm{ICl}$ compared to platinumbased chemotherapy (HR: $0.56,95 \% \mathrm{Cl} 0.43$ to $0.73, \mathrm{P}<0.001, \mathrm{I} 2=$ 0\%, 2 RCTs, 433 participants) Analysis 2.3.

The pooled results showed no evidence of a difference on PFS between the two groups (HR: $0.86,95 \% \mathrm{Cl} 0.53$ to $1.40 ; \mathrm{P}=0.55, \mathrm{I}^{2}$ $=91 \%, 2 \mathrm{RCTs}, 1202$ participants) Analysis 2.3. There was evidence of a difference between the two subgroups (Test for subgroup differences: $\left.\mathrm{Chi}^{2}=13.29, \mathrm{df}=1(\mathrm{P}=0.0003), \mathrm{I}^{2}=92.5 \%\right)$ Analysis 2.3 .

3. Secondary outcomes - Overall response rate (ORR)

- a. Main analysis: ORR by PD-L1 expression

No studies found reported on this outcome. 
- b. Subgroup analyses:.

- i. ORR by Tumour Mutational Burden (TMB)

Two included trials (Hellmann 2018; Rizvi 2020) provided data for this outcome.

TMB - Low. Only Rizvi 2020, provided ORR data for people with blood TMB $<20$ mut/Mb. There was evidence of a difference with a higher ORR with chemotherapy treatment compared to double $\mathrm{ICl}$ (RR: $0.53,95 \% \mathrm{Cl} 0.37$ to $0.77, \mathrm{P}<0.001,1 \mathrm{RCT}, 389$ participants).

TMB - High. Both included trials provided ORR data for people with high TMB. There was evidence of a difference between the two groups with a lower ORR in people treated with platinum-based chemotherapy compared to double ICI (RR $1.83,95 \% \mathrm{Cl} 1.40$ to 2.39 , $\mathrm{I}^{2}=0 \%, \mathrm{P}<0.0001,2 \mathrm{RCTs}, 433$ participants) Analysis 2.4
Overall, there was no evidence of a difference between the two groups (RR 1.25, 95\% Cl 0.53 to $2.96 ; \mathrm{I}^{2}=93 \%, \mathrm{P}=0.60,2$ RCTs, 822 participants) Analysis 2.4. There was evidence of a difference between TMB low and high subgroups (Test for subgroup differences: Chi $^{2}=27.65, \mathrm{df}=1(\mathrm{P}<0.00001)$ Analysis 2.4.

\section{Secondary outcome - Adverse events (AEs)}

Two trials comparing double $\mathrm{ICI}$ to platinum-based chemotherapy (Rizvi 2020; Hellmann 2018) reported grade 3 to 5 AEs. The pooled results showed no evidence of a difference between the two groups in grade 3 to 5 adverse events ( $R R 0.84,95 \% \mathrm{Cl} 0.62$ to $1.15 ; \mathrm{P}=0.28$, $\mathrm{I}^{2}=60 \%, 2 \mathrm{RCTs}, 3738$ participants) Analysis 2.5; Figure 9. Applying a fixed-effect model there evidence of a difference between in favour of people treated with double ICI (RR $0.83,95 \% \mathrm{Cl} 0.73$ to $0.95 ; \mathrm{P}=$ $0.007,12=60 \%, 3738$ participants) (analysis not shown).

Figure 9. Forest plot of comparison: Combined Immuno versus Chemotherapy, outcome: 5.5 Adverse Events grade 3-5.

\begin{tabular}{|c|c|c|c|c|c|}
\hline \multirow[b]{2}{*}{ Study or Subgroup } & \multicolumn{2}{|c|}{ Immunotherapy } & \multicolumn{2}{|c|}{ Chemotherapy } & \multirow[b]{2}{*}{ Weight } \\
\hline & Events & Total & Events & Total & \\
\hline \multicolumn{6}{|c|}{ 2.5.1 Adverse events grade 3-4 } \\
\hline Hellmann 2018 & 189 & 576 & 205 & 570 & $47.5 \%$ \\
\hline Rizvi 2020 & 79 & 371 & 116 & 352 & $40.5 \%$ \\
\hline Subtotal (95\% CI) & & 947 & & 922 & $88.0 \%$ \\
\hline Total events: & 268 & & 321 & & \\
\hline \multicolumn{6}{|c|}{ Heterogeneity: $\mathrm{Tau}^{2}=0.05 ; \mathrm{Chi}^{2}=5.33, \mathrm{df}=1(\mathrm{P}=0.02) ; \mathrm{I}^{2}=81 \%$} \\
\hline \multicolumn{6}{|c|}{ Test for overall effect: $\mathrm{Z}=1.46(\mathrm{P}=0.15)$} \\
\hline \multicolumn{6}{|c|}{ 2.5.2 Adverse events grade 5 (toxic deaths) } \\
\hline Hellmann 2018 & 8 & 576 & 6 & 570 & $7.4 \%$ \\
\hline Rizvi 2020 & 6 & 371 & 3 & 352 & $4.6 \%$ \\
\hline Subtotal $(95 \%$ CI $)$ & & 947 & & 922 & $12.0 \%$ \\
\hline Total events: & 14 & & 9 & & \\
\hline \multicolumn{6}{|c|}{ Heterogeneity: $\mathrm{Tau}^{2}=0.00 ; \mathrm{Chi}^{2}=0.17, \mathrm{df}=1(\mathrm{P}=0.68) ; \mathrm{I}^{2}=0 \%$} \\
\hline \multicolumn{6}{|c|}{ Test for overall effect: $\mathrm{Z}=0.96(\mathrm{P}=0.34)$} \\
\hline Total $(95 \%$ CI $)$ & & 1894 & & 1844 & $100.0 \%$ \\
\hline Total events: & 282 & & 330 & & \\
\hline \multicolumn{6}{|c|}{ Heterogeneity: $\mathrm{Tau}^{2}=0.05 ; \mathrm{Chi}^{2}=7.44, \mathrm{df}=3(\mathrm{P}=0.06) ; \mathrm{I}^{2}=60 \%$} \\
\hline \multicolumn{6}{|c|}{ Test for overall effect: $\mathrm{Z}=1.08(\mathrm{P}=0.28)$} \\
\hline Test for subgroup & Chi & & $=0$. & & \\
\hline
\end{tabular}

Risk Ratio $\quad$ Risk Ratio

M-H, Random, $95 \%$ CI
Adverse Events grade 3 to 4. There was no evidence of a difference in grade 3 and 4 AEs among people receiving double $\mathrm{ICl}$ compared to platinum-based chemotherapy (RR: 0.78, 95\% $\mathrm{Cl} 0.55$ to $1.09, \mathrm{P}=0.15, \mathrm{l}=81 \%, 2 \mathrm{RCTs}, 1869$ participants, low certainty of evidence) Analysis 2.5 ; Figure 9. The certainty for this outcomes was downgraded one level due to study limitations, and one level due to inconsistency. However, there was evidence of a difference between the two groups favouring double $\mathrm{ICI}$ compared to platinum-based chemotherapy when we applied a fixed-effect model (RR: $0.81,95 \% \mathrm{Cl} 0.71$ to $0.93, \mathrm{P}=0.003, \mathrm{I} 2=81 \%, 2 \mathrm{RCT}, 1869$ participants) (analysis not shown)

Adverse Events grade 5 (toxic deaths). There was no evidence of a difference in participants who received double-agent $\mathrm{ICI}$ compared to platinum-based chemotherapy in the risk of toxic death (RR: 1.51,
$95 \% \mathrm{Cl} 0.65$ to $3.48, \mathrm{P}=0.33, \mathrm{I}^{2}=0 \%, 2 \mathrm{RCTs}, 1869$ participants) Analysis 2.5 ; Figure 9.

There was no evidence of a difference between the two subgroups (Test for subgroup differences: $\mathrm{Chi}^{2}=2.07, \mathrm{df}=1(\mathrm{P}=0.15), \mathrm{I}^{2}=$ $51.8 \%)$.

\section{Secondary outcome - Health-related quality of life (HRQoL)}

None of the included trials reported this outcome.

\section{DISCUSSION}

\section{Summary of main results}

We obtained data for 5893 participants from seven studies comparing first-line single-agent (six trials) or double-agent (two trials) immune checkpoint inhibitors (ICI) with platinum-based

Single or combined immune checkpoint inhibitors compared to first-line platinum-based chemotherapy with or without bevacizumab for $\mathbf{2 7}$ people with advanced non-small cell lung cancer (Review)

Copyright (c) 2020 The Cochrane Collaboration. Published by John Wiley \& Sons, Ltd. 
chemotherapy; one study comparing both first-line, single- and combined ICl with platinum-based chemotherapy.

\section{Single-agent immune checkpoint inhibitors (ICIs) versus chemotherapy}

Overall, the effect of first-line, single-agent $\mathrm{ICl}$ on survival (overall survival (OS) and progression-free (PFS)) and responses (objective response rate (ORR)) were not consistent across different PDL1 subgroups (negative, positive, high). In fact, for people with advanced non-small lung cell cancer (NSCLC) and PD-L1 expression $\geq 50 \%$, single-agent $\mathrm{ICI}$ improved OS, PFS and ORR compared to platinum-based chemotherapy, while for people with PD-L1 expression $<1 \%$ or $\geq 1 \%$ no differences between single-agent $\mathrm{ICl}$ and platinum-based chemotherapy were observed. For some outcomes, such as OS, the absence of a survival difference in PD$L 1 \geq 1 \%$ subgroup could be explained by the high heterogeneity coming from studies with imbalance in baseline characteristics between treatment groups or with a high cross-over rate from platinum-based chemotherapy to single-agent ICI. Similarly, the high heterogeneity observed for PFS or ORR in PD-L1 $1 \%$ and in PD-L1 $\geq 50 \%$ subgroups, respectively, could be explained by the different methods used to determine PD-L1 expression on tumour cells or immune cells, or by the repeated PD-L1 testing performed in some trials.

Subgroups analysis according to PD-L1 expression and baseline clinical characteristics of participants of the included studies showed absence of a difference according to age, gender, ECOG PS (Eastern Cooperative Oncology Group Performance Status) or histology categories. In fact, single-agent $\mathrm{ICl}$ improved OS compared to platinum-based chemotherapy in people with PD$\mathrm{L} 1 \geq 50 \%$ regardless of age, gender, ECOG PS and histology while no difference between the two treatments was found in people with PD-L1 expression $\geq 1 \%$. The only clinical characteristic with a potential impact on OS was smoking status in people with PD-L1 expression $\geq 50 \%$. In fact, in people who had never smoked with PD$\mathrm{L} 1$ expression $\geq 50 \%$ there was no difference in OS between singleagent $\mathrm{ICl}$ and platinum-based chemotherapy, while in current and former smokers single-agent $\mathrm{ICl}$ improved OS compared to platinum-based chemotherapy.

Overall, in people with evaluable tumour mutational burden (TMB), single-agent $\mathrm{ICl}$ did not improve survival (OS and PFS) nor response (ORR) compared to platinum-based chemotherapy. However, results were not consistent according to different TMB subgroups (high versus low) and in people with high TMB, singleagent $\mathrm{ICI}$ improved OS and PFS compared to platinum-based chemotherapy while in people with low TMB, platinum-based chemotherapy was superior to single-agent $\mathrm{ICl}$ in terms of ORR and $\mathrm{PFS}$, and no difference between these two treatments was observed in OS.

Single-agent $\mathrm{ICl}$ was associated with reduced grade 3 to 5 adverse events (AEs) compared to platinum-based chemotherapy. However, a difference according to AEs grade subgroups (grade 3 to 4 versus grade 5) was observed and fewer grade 3 to $4 \mathrm{AEs}$ were reported with single-agent $\mathrm{ICI}$ while no difference in toxic deaths (grade $5 \mathrm{AEs}$ ) between single-agent $\mathrm{ICl}$ and platinum-based chemotherapy was found. AEs were not reported according to PDL1 expression and the high heterogeneity observed for grade 3 to 4 AEs may be due to the inclusion of people with different PD-
L1 expression levels who could also experience different degree of toxicities from single-agent $\mathrm{ICl}$.

Data regarding health-related quality of life (HRQoL) came from one single study and showed no difference or a better performance in QOL-C30 and QLQ-LC13 domains for pembrolizumab compared to platinum-based chemotherapy with the exception of diarrhoea showing a higher proportion of people with less diarrhoea at week 15 in the chemotherapy group compared to single-agent ICl.

\section{Combined ICls versus chemotherapy}

Overall, the effect of combined ICIs on OS compared to platinumbased chemotherapy were not consistent across different PD-L1 or TMB subgroups. double-agent ICls prolonged OS compared to platinum-based chemotherapy in people with PD-L1 expression $\geq$ $50 \%$ or $<1 \%$ or with high TMB while no differences in OS between treatments were observed in people with PD-L1 expression $\geq 1 \%$ or with low TMB. Overall, combined ICIs did not improve PFS and ORR compared to platinum-based chemotherapy but a subgroup effect was observed with improved PFS and ORR in people with high TMB and no difference between treatments or improved ORR or PFS with chemotherapy compared to combined ICIs in people with low TMB.

No difference in grade 3 to 5 adverse events between combined ICls and platinum-based chemotherapy was observed.

\section{Overall completeness and applicability of evidence}

The following limitations may affect the strength of conclusions of this review.

- There are no data available to explore the effect of single- or combined ICls compared to platinum-based chemotherapy in special subgroups such as people with brain or liver metastases or with common oncogenic drivers in NSCLC (i.e. KRAS, EGFR and BRAF mutations or ALK and ROS1 rearrangements).

- We did not explore the role of chemotherapy-immunotherapy combinations because another Cochrane Review is currently ongoing on this topic (Syn 2018).

- Subgroup analyses according to clinical characteristics for the effectiveness of double- $\mathrm{ICl}$ treatment compared to platinumbased chemotherapy was not performed because data were only available for one trial (Hellmann 2018).

- The subgroup analyses for the comparison of single-agent ICI with chemotherapy according to other clinical characteristics (age, gender, histology, ECOG PS, smoking status) involved only a small number of people and could not lead to definitive conclusions.

- Analysis of HRQoL was only reported in only one study (Reck 2016) and was missing for the studies comparing combined ICls versus platinum-based chemotherapy.

- The applicability of the evidence about PD-L1 as biomarker for $\mathrm{ICl}$ effectiveness is limited by the fact that PD-L1 expression in one out of six studies (Herbst 2020) was measured on both tumour and immune cells and that in the same study the antibody for PD-L1 staining (SP142) had lower sensitivity compared to the others (Tsao 2018). Moreover we could not draw any conclusions about the effectiveness of single-agent $\mathrm{ICl}$ compared to platinum-based chemotherapy because PD-L1 expression $<1 \%$ as negative PD-L1 was an exclusion criterion in five out of six trials comparing single-agent $\mathrm{ICl}$ versus platinum-based chemotherapy. However, the survival data for

Single or combined immune checkpoint inhibitors compared to first-line platinum-based chemotherapy with or without bevacizumab for 28 people with advanced non-small cell lung cancer (Review)

Copyright (c) 2020 The Cochrane Collaboration. Published by John Wiley \& Sons, Ltd. 
this subgroup were available for the comparison of double-ICI treatment with platinum-based chemotherapy.

- TMB analyses across different studies were mainly exploratory and included too few participants to produce generalisable results. In addition, the different samples (tissue in Carbone 2017, Mok 2019 and Hellmann 2018 and plasma in (Herbst 2020), methodologies (whole exome sequencing in Carbone 2017 and Mok 2019, Foundation one CDx tissue assay in Hellmann 2018, Guardant OMNI next generation sequencing (NGS) assay in Rizvi 2020. Foundation One plasma NGS assay in Herbst 2020) and thresholds used ( $\geq 243$ mut/exome in Carbone 2017, $\geq 175$ mut/exome in Mok 2019, $\geq 10$ Mut/Mb in Hellmann 2018 and $\geq 20$ Mut/Mb in Rizvi 2020 and in Herbst 2020) may limit the generalisability of the results. Another possible limitation of this meta-analysis is that results for subgroups defined by TMB status were mainly exploratory and computed on sample sizes too small to derive definite and generalisable conclusions.

- We could not provide enough information about potential harmful effects of single- or combined ICls. In fact, recent evidence has shown a possible detrimental effect of $\mathrm{ICl}$, known as hyper progressive disease (HPD) and characterised by tumour growth acceleration, rapid progression and deaths in a subgroup of people with advanced NSCLC treated with single-agent $\mathrm{ICI}$ (Champiat 2018; Ferrara 2018). Although HPD has not been explored in clinical trials, the early mortality rate in the first three months could approximately estimate the potential detrimental effect of ICl in people with NSCLC (Ferrara 2020).

\section{Quality of the evidence}

All the included studies were randomised parallel controlled trials, and cross-over was permitted in three trials (Carbone 2017, Reck 2016, Sezer 2020). Because of the lack of information about one trial (Sezer 2020), only available as an abstract, three domains of the risk of bias remain unclear. All trials were potentially affected by performance bias because of their open-label design, but five trials (Carbone 2017; Hellmann 2018; Reck 2016; Rizvi 2020; Sezer 2020) in which PFS was a primary endpoint were at high risk of performance bias due to the lack of blinding of participants and personnel, whereas in two studies (Herbst 2020; Mok 2019 ) in which the primary endpoint was OS, performance bias influenced only the results of secondary or exploratory outcomes. Six trials were at low risk of detection bias because OS was the primary endpoint or when PFS was a primary endpoint, the outcome assessors were blinded to the treatment assignment. Two trials (Hellmann 2018; Rizvi 2020) were also at high risk of attrition bias because of the high dropout rate of people after randomisation. The risk of detection and of attrition bias was not clear for Sezer 2020 , because it was available only as an abstract. Four studies were at high risk of other bias because of the imbalance of some baseline characteristics between treatment groups (Carbone 2017), because of amendments affecting primary or secondary endpoints (Carbone 2017; Hellmann 2018; Mok 2019), or because of changes in the primary target population (Sezer 2020). All trials were at unclear risk of other sources of bias because some authors had declared personal fees or other support from the pharmaceutical companies conducting the trials and it was impossible to know how these conflicts of interests have biased data collection and analysis. Publication bias is unlikely to have occurred for the four trials comparing single-agent $\mathrm{ICl}$ compared with platinum-based chemotherapy for both PD-L1 $\geq 1 \%$ and $\geq 50 \%$ categories, while it was not possible to assess publication bias for the comparison double $\mathrm{ICI}$ versus chemotherapy because of the limited number of studies included.

Overall survival results were reliable, because of absence of detection bias and because performance bias affected only some trials despite the open-label design of all the studies. Because of attrition bias and the high risk of other source of bias in some trials, we could not derive to definitive conclusions from the survival data for specific subgroups according to TMB status and PD-L1 expression.

Using GRADE assessment, the certainty of the evidence ranged from moderate to low (see Summary of findings 1; Summary of findings 2). Moderate certainty of evidence was due to the high risk of attrition bias, performance bias or other sources of bias affecting some of the included studies. Certainty of evidence was downgraded for some outcomes because of inconsistency of results and a high heterogeneity score. Certainty of evidence for outcomes coming from one single study (i.e. HRQoL for single-agent $\mathrm{ICI}$ compared to chemotherapy in Reck 2016 and ORR of combined ICI compared to chemotherapy by TMB in Rizvi 2020), or having results with wide confidence intervals was further downgraded because of the imprecision.

\section{Potential biases in the review process}

We implemented a wide search that included ongoing trial registry databases and contacted experts in the field. We did not apply any language or publication status restrictions. Therefore, we are certain that all the relevant trials were included in the review so far. It is unknown whether there are other reports of unpublished trials in other languages or presented at different meetings. However, the living systematic approach will allow us to integrate emerging evidence to the existing one, this will limit potential biases due to the review process. We prespecified all the review outcomes and subgroups prior to the completion of the analysis. However, it was not feasible to investigate the risk of publication bias and complete a funnel plot due to the insufficient number of included studies.

\section{Agreements and disagreements with other studies or reviews}

Different systematic reviews and network meta-analyses have recently investigated the use of first-line chemotherapy and immunotherapy combinations in NSCLC (Chen 2019; Dafni 2019), or the effectiveness of $\mathrm{ICl}$ regardless to treatment type (alone or in combination) and line of therapy (Sun 2020; Yu 2019). This is the first meta-analysis exploring single- and combined $\mathrm{ICl}$ treatment compared with platinum-based chemotherapy in first-line setting.

Other meta-analyses including different cancer types or NSCLC people treated with $\mathrm{ICl}$ in different lines and combinations have explored the benefit of $\mathrm{ICl}$ according to clinical relevant characteristics. Unlike our meta-analysis, single-agent $\mathrm{ICI}$ did not significantly improve OS compared to first-line chemotherapy in elderly ( $\geq 65$ years) people (Raphael 2020) in one meta-analysis. However data were not stratified according to PD-L1 expression and only three studies using first-line $\mathrm{ICl}$ were included. The same meta-analysis showed no OS and PFS benefit for first-line single-agent $\mathrm{ICl}$ compared to chemotherapy among people who never smoked, being in line with our findings for this specific subgroup. In this regard, never/light smoker status has been recently associated with lower PFS and duration of response 
following single-agent $\mathrm{ICl}$ compared to heavy smoking in people with advanced NSCLC and PD-L1 expression $\geq 50 \%$ (Gainor 2020). Similarly, never smoking status is often associated with oncogenic drivers potentially targetable with active drugs and in a multicentric retrospective registry single-agent $\mathrm{ICl}$ showed low clinical activity in patients with actionable molecular alterations (Mazieres 2019).

A recent meta-analysis of three trials showed that pembrolizumab improved OS compared to platinum-based chemotherapy in people with advanced NSCLC older than 75 years and similarly to the present study, the improvement was significant only in people with PD-L1 expression $\geq 50 \%$. This finding suggests that PD-L1 status rather than age has an impact on survival upon single-agent ICl (Nosaki 2019).

Although we did not find a subgroup effect according to sex, single-agent $\mathrm{ICl}$ did not improve survival compared to platinumbased chemotherapy in females with PD-L1 expression $>1 \%$. In agreement with us, the lower magnitude of benefit of single-agent $\mathrm{ICl}$ in women across different cancer types has been reported in a recent meta-analysis (Conforti 2018). Furthermore, a metaanalysis of eight trials comparing single-agent $\mathrm{ICI}$ to chemotherapy in both treatment naive and previously treated people with advanced NSCLC have reported no significant benefit in PFS among females (El-Osta 2019).This finding was not confirmed by another meta-analysis (Wallis 2019), however, it also included studies testing combinations of $\mathrm{ICl}$ and anti-CTLA-4 agents or $\mathrm{ICl}$ and chemotherapy.

Finally, we reported that both high PD-L1 or high TMB are associated with increased benefit from $\mathrm{ICl}$ as single agents or in combination compared to platinum-based chemotherapy. Similarly, PD-L1 and TMB were independently associated with better response and survival outcome in another meta-analysis including $\mathrm{ICl}$ monotherapy or in combination with other treatment (Yu 2019).

\section{AUTHORS' CONCLUSIONS}

\section{Implications for practice}

The evidence in this review suggests that single-agent $\mathrm{ICI}$ in people with NSCLC and PD-L1 $\geq 50 \%$ probably leads to a higher overall survival rate and may lead to a higher progression free survival and overall response rate when compared to platinumbased chemotherapy and may also lead to a lower rate of adverse events and higher HRQoL. Combined ICI in people with NSCLC and $\mathrm{PD}$-L1 $\geq 50 \%$ also probably leads to a higher overall survival rate when compared to platinum-based chemotherapy, but its effect on progression free survival, overall response rate and $\mathrm{HRQ}$ L $\mathrm{L}$ is unknown due to a lack of data. The rate of adverse events may not differ between groups.

\section{Implications for research}

The characterisation of both molecular profiles and tumour microenvironment from people who have never smoked could improve the understanding of mechanisms of resistance to $\mathrm{ICl}$. In addition, our findings suggest to integrate multiple clinical and biological (i.e. TMB) parameters together with PD-L1 expression in a common algorithm able to predict effectiveness of single- or double-agent ICls in people with advanced NSCLC.

\section{ACK N OWLEDGEMENTS}

We thank Joanne Brooker, Tari Turner, Nuala Livingston and Eve Tomlinson for methodological support.

We thank Giorgio Maria Agazzi for designing the Embase search strategy ; Cheryl Ho, Sarah Hodgkinson, Fergus Macbeth, Celine Mascaux, Nichole Taske, Marta Roque Figuls and Anne-Claire Toffart for comments on our protocol, as well as Nicole Skoetz, Sign-off Editor. 


\section{R E F E R E N C E S}

\section{References to studies included in this review}

\section{Carbone 2017 \{published data only\}}

* Carbone DP, Reck M, Paz-Ares L, Creelan B, Horn L, Steins M. First-line nivolumab in stage IV or recurrent non-small-cell lung cancer. New England Journal of Medicine 2017;376:2415-26.

NCT02041533. An open-label, randomized, phase 3 trial of nivolumab versus investigator's choice chemotherapy as firstline therapy for stage IV or recurrent PD-L1+ non-small cell lung cancer (CheckMate 026). https://clinicaltrials.gov/ct2/show/ NCT02041533 accessed 27 May 2019.

\section{Hellmann 2018 \{published data only\}}

Brahmer J, Schenker M, Lee KH, Provencio M, Nishio M, Lesniewski-Kmak K, et al. CheckMate 227: patient-reported outcomes of first-line nivolumab + ipilimumab in high tumor mutational burden advanced NSCLC. Journal of Thoracic Oncology 2018;13(suppl 10):S332.

* Hellmann MD, Ciuleanu TE, Pluzanski A, Lee JS, Otterson GA, Audigier-Valette $C$, et al. Nivolumab plus ipilimumab in lung cancer with a high tumor mutational burden. New England Journal of Medicine 2018 May 31;378(22):2093-104. [DOI: 10.1056/NEJMoa1801946]

Hellmann MD, Paz-Ares L, Bernabe Caro R, Zurawski B, Kim SW, Carcereny Costa E. Nivolumab plus ipilimumab in advanced non-small-cell lung cancer. New England Journal of Medicine 2019;381:2020-31. [DOI: 10.1056/NEJMoa1910231]

O'Byrne KJ, Lee KH, Kim SW, Park K, Nishio M, Sakai H, et al. First-line (1L) nivolumab (NIVO) plus ipilimumab (IPI) in Asian patients (pts) with advanced non-small cell lung cancer (aNSCLC) in CheckMate 227. Annals of Oncology 2020;31(suppl 4):S824

Paz-Ares L, Brahmer J, Hellmann MD, Reck M, O'Byrne K, Borghaei $\mathrm{H}$, et al. CheckMate 227: a randomized, open-label phase 3 trial of nivolumab nivolumab plus ipilimumab, or nivolumab plus chemotherapy versus chemotherapy in chemotherapy-naive patients with advanced non-small cell lung cancer (NSCLC). Annals of Oncology 2017;28:iii50-1.

Peters S, Ramalingam SS, Paz-Ares L, Bernabe Caro R, Zurawski B, Kim SW, et al. Nivolumab (NIVO) + low-dose ipilimumab (IPI) vs platinum-doublet chemotherapy (chemo) as first-line ( $1 \mathrm{~L}$ ) treatment (tx) for advanced non-small cell lung cancer (NSCLC): CheckMate 227 part 1 final analysis. Annals of Oncology 2019;30(suppl 5):v913-4.

Ramalingam SS, Ciuleanu TE, Pluzanski A, Lee JS, Schenker M, Caro RB, et al. Nivolumab + ipilimumab versus platinumdoublet chemotherapy as first-line treatment for advanced non-small cell lung cancer: Three-year update from CheckMate 227 Part 1. Journal of Clinical Oncology May 20, 2020;38, no. 15_suppl:9500.

Reck M, Hellmann MD, Paz-Ares LG, Ramalingam SS, Brahmer JR, O'Byrne KJ, et al. Nivolumab (nivo) + ipilimumab (Ipi) vs platinum-doublet chemotherapy (chemo) as first-line
(1L) treatment (Tx) for advanced non-small cell lung cancer (NSCLC): safety analysis and patient-reported outcomes (PROs) from CheckMate 227. Journal of Clinical Oncology 2018;36(15):9020.

\section{Herbst 2020 \{published data only\}}

A study of atezolizumab (MPDL3280A) compared with a platinum agent (cisplatin or carboplatin) + (pemetrexed or gemcitabine) in participants with stage IV non-squamous or squamous non-small cell lung cancer (NSCLC) [IMpower110]. https://clinicaltrials.gov/ct2/show/NCT02409342 accessed May 2019.

De Marinis F, Jassem J, Spigel DR, Lam S, Mocci S, Sandler A, et al. 480TiP - IMpower110: Phase III study on $1 \mathrm{~L}$ atezolizumab (atezo) in PD-L1-selected chemotherapy (chemo)-naive NSCLC patients (pts). In: Annals of Oncology. Vol. 27 (suppl 9). 2016:ix139-ix56.. [DOI: 10.1093/annonc/mdw594]

De Marinis F, Jassem J, Spigel DR, Lam S, Mocci S, Sandler A, et al. PIMpower110: Phase III study on $1 \mathrm{~L}$ atezolizumab (atezo) in PD-L1-selected chemotherapy (chemo)-naive NSCLC patients (pts). Annals of Oncology 2016;27(Suppl 9):ix139-56.

Herbst RS, de Marinis F, Giaccone G, Reinmuth N, Vergnenegre A, Barrios $\mathrm{CH}$. Clinical efficacy of atezolizumab (atezo) in biomarker subgroups by SP142, SP263 and 22C3 PD-L1 immunohistochemistry (IHC) assays and by blood tumour mutational burden (bTMB): results from the IMpower110 study. In: Annals of Oncology. Vol. Abstract presented at Proffered Paper session, ESMO Immunoncology Congress. 2019.

* Herbst RS, Giaccone G, de Marinis F, Reinmuth N, Vergnenegre A, Barrios $\mathrm{CH}$, et al. Atezolizumab for first-line treatment of PD-L1-selected patients with NSCLC. New England Journal of Medicine Oct 2020;383(14):1328-39.

Spigel D, de Marinis F, Giaccone G, Reinmuth N, Vergnenegre A, Barrios CH. IMpower110: Interim overall survival (OS) analysis of a phase III study of atezolizumab (atezo) vs platinum-based chemotherapy (chemo) as first-line (1L) treatment (tx) in PD-L1selected NSCLC. Annals of Oncology (2019);30 (suppl_5):v851v934. 10.1093/annonc/mdz394.

\section{Mok 2019 \{published data only\}}

Lopes G, Wu YL, Kudaba I, Kowalski D, Cho BC, Castro G, et al. Pembrolizumab (pembro) versus platinum based chemotherapy (chemo) as first-line therapy for advanced/ metastatic NSCLC with a PD-L1 tumor proportion score (TPS) $\geq 1 \%$ : open-label, phase 3. Journal of Clinical Oncology 2018;36(18):LBA4.

* Mok TS, Wu YL, Kudaba I, Kowalski DM, Cho BC, Turna HZ, et al. Pembrolizumab versus chemotherapy for previously untreated, PD-L1-expressing, locally advanced or metastatic non-small-cell lung cancer (KEYNOTE-042): a randomised, openlabel, controlled, phase 3 trial. Lancet 2019;393(10183):1819-30.

\section{Reck 2016 \{published data only\}}

Brahmer J, Rodriguez-Abreu D, Robinson A, Hui R, Csoszi T, Fulop A, et al. Updated analysis of keynote-024: pembrolizumab

Single or combined immune checkpoint inhibitors compared to first-line platinum-based chemotherapy with or without bevacizumab for $\mathbf{3 1}$ people with advanced non-small cell lung cancer (Review)

Copyright (c) 2020 The Cochrane Collaboration. Published by John Wiley \& Sons, Ltd. 
vs platinum-based chemotherapy for advanced NSCLC with PDL1 TPS >=50\%. Journal of Thoracic Oncology 2017;12(11 suppl 2):S1793-4

Brahmer JR, Rodriguez-Abreu D, Robinson AG, Hui R, Csoszi T, Fulop A, et al. Health-related quality-of-life results for pembrolizumab versus chemotherapy in advanced, PD-L1positive NSCLC (KEYNOTE-024): a multicentre, international, randomised, open-label phase 3 trial. The Lancet Oncology 2017;18(12):1600-9.

Brahmer JR, Rodriguez-Abreu D, Robinson AG, Hui R, Csoszi T, Fulop A, et al. KEYNOTE-024 5-year OS update: first-line (1L) pembrolizumab (pembro) vs platinum-based chemotherapy (chemo) in patients (pts) with metastatic NSCLC and PD-L1 tumour proportion score (TPS) $>=50 \%$. Annals of Oncology 2020;31(suppl 4):S1181-2.

* Reck M, Rodríguez-Abreu D, Robinson AG, Hui R, Csőszi T, Fülöp A, et al. KEYNOTE-024 Investigators. pembrolizumab versus chemotherapy for PD-L1-Positive non-small-cell lung cancer. New England Journal of Medicine 2016;375(19):1823-33.

Reck M, Rodríguez-Abreu D, Robinson AG, Hui R, Csőszi T, Fülöp A, et al. Updated analysis of KEYNOTE-024: pembrolizumab versus platinum-based chemotherapy for advanced non-small-cell lung cancer with PD-L1 tumor proportion score of $50 \%$ or greater. Journal of Clinical Oncology 2019 March;37(7):537-46.

Satouchi M, Nosaki K, Takahashi T, Nakagawa K, Aoe K, Kurata T, et al. First-line pembrolizumab versus chemotherapy in metastatic non-small-cell lung cancer: KEYNOTE-024 Japan subset. Cancer Science 2020;00:1-10.

\section{Rizvi 2020 \{published data only\}}

Cho BC, Lee KH, Ahn M-J, Lucien Geater S, Ngoc TV, Wang C-C, et al. Efficacy and safety of first-line durvalumab (D) \pm tremelimumab (T) vs chemotherapy (CT) in Asian patients with metastatic NSCLC: results from MYSTIC. Annals of Oncology 2019;30:ix157-8.

NCT02453282. Phase III open label first line therapy study of MEDI 4736 (durvalumab) with or without tremelimumab versus SOC in non small-cell lung cancer (NSCLC). (MYSTIC). available at https://clinicaltrials.gov/ct2/show/NCT02453282.

Peters S, Chul Cho B, Reinmuth N, Lee KH, Luft A, Ahn MJ, et al. Tumor mutational burden (TMB) as a biomarker of survival in metastatic non-small cell lung cancer (mNSCLC): Blood and tissue TMB analysis from MYSTIC, a phase III study of firstline durvalumab \pm tremelimumab vs chemotherapy. Cancer Research 2019;79 (13 Suppl):Abstract nr CT074.

* Rizvi NA, Cho BC, Reinmuth N, Lee KH, Luft A, Ahn MJ, et al. Durvalumab with or without tremelimumab vs standard chemotherapy in first-line treatment of metastatic nonsmall cell lung cancer: the MYSTIC Phase 3 randomized clinical trial. JAMA Oncology 2020;Apr 9:E1-14. [DOI: 10.1001/ jamaoncol.2020.0237]

Rizvi NA, Chul Cho B, Reinmuth N, Lee KH, Ahn M, Luft A, et al. Durvalumab with or without tremelimumab vs platinumbased chemotherapy as first-line treatment for metastatic non- small cell lung cancer: MYSTIC. Annals of Oncology (2018);29 (suppl_10):x39-x43. 10.1093/annonc/mdy511.

\section{Sezer 2020 \{unpublished data only\}}

NCT03088540. A global, randomised, phase 3, open-label study of REGN2810 (ANTI-PD 1 Antibody) versus platinum based chemotherapy in first line treatment of patients with advanced or metastatic PD L1+non-small cell lung cancer. https://clinicaltrials.gov/ct2/show/NCT03088540 accessed January 2020.

* Sezer A, Kilickap S, Gümüş M, Bondarenko I, Özgüroğlu M, Gogishvili M, et al. EMPOWERLung 1: Phase 3 first line (1L) Cemiplimab monotherapy vs platinum doublet chemotherapy (chemo) in advanced non small cell lung cancer (NSCLC) with programmed cell death ligand 1 (PD L1) $\geq 50 \%$. In: ESMO Congress. 2020.

\section{References to studies excluded from this review}

Huan 2019 \{published data only\}

Huang M, Pietanza MC, Samkari A, Pellissier J, Burke T, Chandwani S, et al. Q-TWiST analysis to assess benefit-risk of pembrolizumab in patients with PD-L1-positive advanced or metastatic non-small cell lung cancer. Pharmacoeconomics 2019;37:105-16.

\section{Hui 2017a \{published data only\}}

Hui R, Garon EB, Goldman JW, Leighl NB, Hellmann MD, Patnaik $A$, et al. Pembrolizumab as first-line therapy for patients with PD-L1-positive advanced non-small cell lung cancer: a phase 1 trial. Annals of Oncology 2017;28(4):874-81.

\section{Leighl 2019 \{published data only\}}

Leighl NB, Hellmann MD, Hui R, Carcereny E, Felip E, Ahn MJ, et al. Pembrolizumab in patients with advanced non-small-cell lung cancer (KEYNOTE-001): 3-year results from an open-label, phase 1 study. Lancet 2019;7(4):347-57.

\section{Mok 2017 \{published data only\}}

Mok T, Johnson M, Garon E, Peters S, Soria J, Wang L, et al. Poseidon: a phase 3 study of first-line durvalumab +/tremelimumab + chemotherapy vs chemotherapy alone in metastatic NSCLC. Journal of Thoracic Oncology 2017;12(11 suppl 2):S1975.

\section{Ready 2019 \{published data only\}}

Ready N, Hellmann MD, Awad MM, Otterson GA, Gutierrez M, Gainor JF, et al. First-line nivolumab plus ipilimumab in advanced non-small-cell lung cancer (CheckMate 568): outcomes by programmed death ligand 1 and tumor mutational burden as biomarkers PST - aheadofprint DBP - 2019/02/21 06:00. Journal of clinical Oncology 2019;37(12):JCO1801042.

\section{References to ongoing studies}

\section{EMPOWER-Lung 2 \{published data only\}}

NCT03515629. REGN2810 (Anti-PD-1 antibody), platinumbased doublet chemotherapy, and ipilimumab (anti-CTLA-4 antibody) versus pembrolizumab monotherapy in patients with

Single or combined immune checkpoint inhibitors compared to first-line platinum-based chemotherapy with or without bevacizumab for 
lung cancer. https://clinicaltrials.gov/ct2/show/NCT03515629 accessed May 2019.

Rizvi N, Lee S, Curtis P, Caldwell W, Gao B, Rietschel P. EMPOWER-Lung 3: a phase 3 study of cemiplimab, ipilimumab and chemotherapy in advanced NSCLC with PD-L1 $<50 \%$. Journal of Thoracic Oncology October 2018 Volume 13;Supplement(10):S931.

\section{ENERGY \{published data only\}}

NCT03351361. Randomized phase III study testing nivolumab and ipilimumab versus a carboplatin based doublet in first line treatment of PS 2 or elderly patients with advanced non-small cell lung cancer (eNERGY). https://clinicaltrials.gov/ct2/show/ NCT03351361 accessed May 2019.

\section{IPSOS \{published data only\}}

Lee SM, Schulz C, Cardona A, Bartakova P, Peters S. Phase III study of atezolizumab (atezo) vs chemotherapy (chemo) in patients (pts) with treatment-naive advanced, recurrent or metastatic NSCLC unsuitable for platinum (plat)-based chemo. Annals of Oncology 2017;28(suppl 5):v460-96.

NCT03191786. A study of atezolizumab compared with chemotherapy in treatment naïve participants with locally advanced or recurrent or metastatic non-small cell lung cancer who are deemed unsuitable for platinum-containing therapy (IPSOS). https://clinicaltrials.gov/ct2/show/NCT03191786 accessed May 2019.

\section{JAVELIN Lung 100 \{published data only\}}

NCT02576574. Avelumab in first-line non-small cell lung cancer (JAVELIN Lung 100). https://clinicaltrials.gov/ct2/show/ NCT02576574 accessed May 2019.

Reck M, Yang C, Postmus PE, Barlesi F, Font EF, Thomas M, et al. JAVELIN Lung 100: updated design of a phase 3 trial of avelumab vs platinum doublet chemotherapy as first-line (1L) treatment for metastatic or recurrent PD-L11 non-smallcell lung cancer (NSCLC). Annals of Oncology 9 September 2017;28(suppl 5):v460-96.

\section{KEYNOTE-598 \{published data only\}}

NCT03302234. Study of pembrolizumab given with ipilimumab or placebo in participants with untreated metastatic nonsmall cell Lung cancer (MK-3475-598/KEYNOTE-598). https:// clinicaltrials.gov/ct2/show/NCT03302234 accessed May 2019.

\section{MILES 5 \{published and unpublished data\}}

A study comparing immunotherapy with chemotherapy in the treatment of elderly patients with advanced NSCLC (MILES-5). Ongoing study. December 20, 2018Estimated study completion date: June 2023. Contact author for more information.

\section{NEPTUNE \{published data only\}}

Mok T, Schmid P, Aren O, Arrieta O, Gottfried M, Jazieh, et al. 192TiP: NEPTUNE: a global, phase 3 study of durvalumab (MEDI4736) plus tremelimumab combination therapy versus standard of care (SoC) platinum-based chemotherapy in the first-line treatment of patients (pts) with advanced or metastatic NSCLC. Journal of Thoracic Oncology 2016;11(4):S140-41.
Mok T, Schmid P, Aren O, Arrieta O, Gottfried M, Jazieh AR, et al. Phase 3, randomised, open-label study of durvalumab (MEDI4736) in combination with tremelimumab versus platinum-based chemotherapy in first-line treatment of patients with advanced or metastatic NSCLC: NEPTUNE. Annals of Oncology 2015;26(Suppl_9):125-47.

Mok T, Schmid P, de Castro G, Syrigos K, Martin C, N amamoto Y, et al. Global, phase 3 study of first-line durvalumab (MEDI4736) + tremelimumab vs standard of care platinum-based chemotherapy in advanced/metastatic NSCLC: NEPTUNE. Annals of Oncology 2016;27(9):mdw594.046.

Mok T, Schmid P, De Castro G, Syrigos K, Martin C, Yamamoto N, et al. P2.06-022 first-line durvalumab plus tremelimumab vs platinum-based chemotherapy for advanced/metastatic NSCLC: phase 3 NEPTUNE study. Journal of Thoracic Oncology 2017;12(1):S1084.

Study of 1st line therapy study of durvalumab with tremelimumab versus SoC in non small-cell lung cancer (NSCLC) (NEPTUNE). https://clinicaltrials.gov/ct2/show/ NCT02542293 accessed May 2019.

\section{PEARL \{published data only\}}

NCT03003962. Study of durvalumab alone or chemotherapy for patients with advanced non small-cell lung cancer. https:// clinicaltrials.gov/ct2/show/NCT03003962 accessed May 2019.

\section{Additional references}

\section{Antonia 2016}

Antonia S, Goldberg SB, Balmanoukian A, Chaft JE, Sanborn RE, Gupta A, et al. Safety and antitumour activity of durvalumab plus tremelimumab in non-small cell lung cancer: a multicentre, phase 1b study. Lancet Oncology 2016;Mar;17(3):299-308.

\section{Borghaei 2015}

Borghaei H, Paz-Ares L, Horn L, Spigel DR, Steins M, Ready NE, et al. Nivolumab versus docetaxel in advanced nonsquamous non-small-cell lung cancer. New England Journal of Medicine 2015;22, 373(17):1627-39.

\section{Brahmer 2015}

Brahmer J, Reckamp KL, Baas P, Crinò L, Eberhardt WE, Poddubskaya $E$, et al. Nivolumab versus docetaxel in advanced squamous-cell non-small-cell lung cancer. New England Journal of Medicine 2015;373(2):123-35.

\section{Bray 2018}

Bray F, Ferlay J, Soerjomataram I, Siegel RL, Torre LA, Jemal A. Global cancer statistics 2018: GLOBOCAN estimates of incidence and mortality worldwide for 36 cancers in 185 countries. CA: A Cancer Journal for Clinicians 2018;68(6):394-424.

\section{Brockhaus 2014}

Brockhaus AC, Bender R, Skipka G. The Peto odds ratio viewed as a new effect measure. Statistics in Medicine 2014;33(28):4861-74. 


\section{Champiat 2018}

Champiat S, Ferrara R, Massard C, Besse B, Marabelle A, Soria JC, et al. Hyperprogressive disease: recognizing a novel pattern to improve patient management. Nature Review Clinical Oncology 2018;15(12):748-62.

\section{Chen 2019}

Chen Y, Zhou Y, Tang L, Peng X, Jiang H, Wang G, et al. Immunecheckpoint inhibitors as the first line treatment of advanced non-small cell lung cancer: a meta-analysis of randomized controlled trials. Journal of Cancer 2019;10(25):6261-8.

\section{Conforti 2018}

Conforti F, Pala L, Bagnardi V, De Pas T, Martinetti M, Viale G, et al. Cancer immunotherapy efficacy and patients' sex: a systematic review and meta-analysis. Lancet Oncology 2018;19(6):737-46.

\section{Dafni 2019}

Dafni U, Tsourti Z, Vervita K, Peters S. Immune checkpoint inhibitors, alone or in combination with chemotherapy, as first-line treatment for advanced non-small cell lung cancer. A systematic review and network meta-analysis. Lung Cancer 2019 Aug;134:127-40.

\section{Deeks 2011}

Deeks JJ, Higgins JP, Altman DG. Chapter 9. Analysing data and undertaking meta-analyses. In Higgins JP, Green S (editors). Cochrane Handbook for Systematic Reviews of Interventions Version 5.1.0 (updated March 2011). Available from www.handbook.cochrane.org.

\section{DerSimonian 1986}

DerSimonian R, Laird N. Meta-analysis in clinical trials. Control Clinical Trials 1986;7(3):177-88.

\section{Eisenhauer 2009}

Eisenhauer EA, Therasse P, Bogaerts J, Schwartz LH, Sargent D, Ford R. New response evaluation criteria in solid tumours: revised RECIST guideline (version 1.1). European Journal of Cancer 2009;45(2):228-47.

\section{Elbourne 2002}

Elbourne DR, Altman DG, Higgins JP, Curtin F, Worthington HV, Vail A. Meta-analyses involving cross-over trials: methodological issues. International Journal of Epidemiology 2002;31(1):140-9.

\section{Elliott 2017}

Elliott JH, Synnot A, Turner T, Simmonds M, Akl EA, McDonald S, et al. Living systematic review: 1 . Introduction - the why, what, when, and how. Journal of Clinical Epidemiology 2017;91:23-30.

\section{El-Osta 2019}

El-Osta H, Jafri S. Predictors for clinical benefit of immune checkpoint inhibitors in advanced non-small-cell lung cancer: a meta-analysis. Immunotherapy 2019;11(3):189-99.

\section{Ferrara 2018}

Ferrara R, Mezquita L, Texier M, Lahmar J, Audigier-Valette C, Tessonnier $\mathrm{L}$, et al. Hyperprogressive disease in patients with advanced non-small cell lung cancer treated with PD-1/PD-L1 inhibitors or with single-agent chemotherapy. JAMA Oncology 2018;4(11):1543-52.

\section{Ferrara 2020}

Ferrara R, Mezquita L, Texier M, Lahmar J, Audigier-Valette C, Tessonnier $\mathrm{L}$, et al. Comparison of fast-progression, hyperprogressive disease, and early deaths in advanced nonsmall-cell lung cancer treated with PD-1/PD-L1 inhibitors or chemotherapy. Journal of Clinical Oncology Precision Oncology July 2020;4:829-40.

\section{Gainor 2020}

Gainor JF, Rizvi H, Jimenez Aguilar E, Skoulidis F, Yeap BY, Naidoo J, et al. Clinical activity of programmed cell death 1 (PD-1) blockade in never, light, and heavy smokers with nonsmall-cell lung cancer and PD-L1 expression $\geq 50 \%$. Annals of Oncology 2020;31(3):404-11.

\section{Garon 2015}

Garon EB, Rizvi NA, Hui R, Leighl N, Balmanoukian AS, Eder JP, et al. Pembrolizumab for the treatment of non-small-cell lung cancer. New England Journal of Medicine 2015;372(21):2018-28.

\section{Gettinger 2016}

Gettinger S, Rizvi NA, Chow LQ, Borghaei H, Brahmer J, Ready N, et al. Nivolumab monotherapy for first-line treatment of advanced non-small-cell lung cancer. Journal of Clinical Oncology 2016;34(25):2980-7.

\section{Hathcock 1993}

Hathcock KS, Laszlo G, Dickler HB, Bradshaw J, Linsley P, Hodes RJ. Identification of an alternative CTLA-4 ligand costimulatory for T cell activation. Science 1993;262:905-7.

\section{Higgins 2002}

Higgins JP, Thompson SG. Quantifying heterogeneity in a metaanalysis. Statistics in Medicine 2002;21(11):1539-58.

\section{Higgins 2011}

Higgins JP, Green S (editors). Cochrane Handbook for Systematic Reviews of Interventions Version 5.1.0 (updated March 2011). The Cochrane Collaboration. Available from handbook.cochrane.org.

\section{Hui 2017}

Hui E, Cheung J, Zhu J, Su X, Taylor MJ, Wallweber HA, et al. T cell costimulatory receptor $\mathrm{CD} 28$ is a primary target for PD-1mediated inhibition. Science 2017;355(6332):1428-33.

\section{Ishida 1992}

Ishida Y, Agata Y, Shibahara K, Honjo T. Induced expression of PD-1, a novel member of the immunoglobulin gene superfamily, upon programmed cell death. EMBO Journal 1992;11:3887-95.

\section{Kluetz 2016}

Kluetz PG, Chingos DT, Basch EM, Mitchell SA. Patient-Reported Outcomes in Cancer Clinical Trials: measuring symptomatic adverse events with the National Cancer Institute's PatientReported Outcomes version of the Common Terminology Criteria for Adverse Events (PRO-CTCAE). American Society of Clinical Oncology Educational Book 2016;35:67-73. 


\section{Lawrence 2013}

Lawrence MS, Stojanov P, Polak P, Kryukov GV, Cibulskis K, Sivachenko A, et al. Mutational heterogeneity in cancer and the search for new cancer-associated genes. Nature 2013;499(7457):214-8.

\section{Lopez-Chavez 2012}

Lopez-Chavez A, Young T, Fages S, Leon L, Schiller JH, Dowlati A, et al. Bevacizumab maintenance in patients with advanced non-small-cell lung cancer, clinical patterns, and outcomes in the Eastern Cooperative Oncology Group 4599 Study: results of an exploratory analysis. Journal Thoracic Oncology 2012;7(11):1707-12.

\section{Mazieres 2019}

Mazieres J, Drilon A, Lusque A, Mhanna L, Cortot AB, Mezquita L, et al. Immune checkpoint inhibitors for patients with advanced lung cancer and oncogenic driver alterations: results from the IMMUNOTARGET Registry. Annals of Oncology 2019;30(8):1321-8.

\section{Nishino 2013}

Nishino M, Giobbie-Hurder A, Gargano M, Suda M, Ramaiya NH, Hodi FS. Developing a common language for tumor response to Immunotherapy: immune-related response criteria using unidimensional measurements. Clinical Cancer Research 2013;19(14):3936-43.

\section{Nosaki 2019}

Nosaki K, Hosomi Y, Saka H, Baas P, de Castro G, Reck M, et al. Safety and efficacy of pembrolizumab (pembro) monotherapy in elderly patients (pts) with PD-L1-positive advanced NSCLC: pooled analysis from KEYNOTE-010, -024, and -042. Annals of Oncology 2019;30 (suppl_2):ii38-ii68. 10.1093/annonc/mdz063.

\section{Novello 2016}

Novello S, Barlesi F, Califano R, Cufer T, Ekman S, Levra MG, et al. Metastatic non-small-cell lung cancer: ESMO clinical practice guidelines for diagnosis, treatment and follow-up. Annals of Oncology 2016;27(Suppl 5):v1-v27.

\section{Okazaki 2013}

Okazaki T, Chikuma S, Iwai Y, Fagarasan S, Honjo T. A rheostat for immune responses: the unique properties of PD- 1 and their advantages for clinical application. Nature Immunology 2013;14(12):1212-8

\section{Osoba 1998}

Osoba D, Rodrigues G, Myles J, Zee B, Pater J. Interpreting the significance of changes in health-related quality-of-life scores. Journal of Clinical Oncology 1998 Jan;16(1):139-44.

\section{Parmar 1998}

Parmar MK, Torri V, Stewart L. Extracting summary statistics to perform meta-analyses of the published literature for survival endpoints. Statistics in Medicine 1998;17(24):2815-34.

\section{Paz-Ares 2013}

Paz-Ares LG, de Marinis F, Dediu M, Thomas M, Pujol JL, Bidoli P, et al. PARAMOUNT: Final overall survival results of the phase III study of maintenance pemetrexed versus placebo immediately after induction treatment with pemetrexed plus cisplatin for advanced nonsquamous non-small-cell lung cancer. Journal of Clinical Oncology 2013;31(23):2895-902.

\section{Peters 2017}

Peters S, Gettinger S, Johnson ML, Jänne PA, Garassino MC, Christoph D, et al. Phase II trial of atezolizumab as first-line or subsequent therapy for patients with programmed deathligand 1-selected advanced non-small-cell lung cancer (BIRCH). Journal of Clinical Oncology 2017, Aug 20;35(24):2781-89.

\section{Raphael 2020}

Raphael J, Batra A, Boldt G, Shah PS, Blachette P, Rodrigues G, et al. Predictors of survival benefit from immune checkpoint inhibitors in patients with advanced non-small-cell lung cancer: a systematic review and meta-analysis. Clinical Lung Cancer 2020;21(2):106-113.e5.

\section{RevMan 2020 [Computer program]}

The Cochrane Collaboration Review Manager (RevMan). Version 5.4. Copenhagen: Nordic Cochrane Centre: The Cochrane Collaboration, 2014.

\section{Scagliotti 2008}

Scagliotti GV, Parikh P, von Pawel J, Biesma B, Vansteenkiste J, Manegold C, et al. Phase III study comparing cisplatin plus gemcitabine with cisplatin plus pemetrexed in chemotherapynaive patients with advanced-stage non-small-cell lung cancer. Journal of Clinical Oncology 2008;26(21):3543-51.

\section{Seymour 2017}

Seymour L, Bogaerts J, Perrone A, Ford R, Schwartz LH, Mandrekar S. iRECIST: guidelines for response criteria for use in trials testing immunotherapeutics. Lancet Oncology 2017;18(3):e143-e152.

\section{Simmonds 2017}

Simmonds M, Salanti G, McKenzie J, Elliott J, on behalf of the Living Systematic Review Network. Living systematic reviews: 3. Statistical methods for updating meta-analyses. Journal of Clinical Epidemilogy 2017;91:38-46.

\section{Sterne 2011}

Sterne JA, Egger M, Moher D. Chapter 10. addressing reporting biases. In: Higgins JP, Green S (editors). Cochrane Handbook for Systematic Reviews of Interventions Version 5.1.0 (updated March 2011). Available from handbook.cochrane.org.

\section{Sun 2020}

Sun L, Zhang L, Yu J, Zhang Y, Pang X, Ma C, et al. Clinical efficacy and safety of anti-PD-1/PD-L1 inhibitors for the treatment of advanced or metastatic cancer: a systematic review and meta-analysis. Scientific Reports 2020;10(1):2083.

\section{Syn 2018}

Syn NL, Roudi R, Wang LZ, Wang L, Loh M, Huang Y, et al. Immune checkpoint inhibitors plus chemotherapy versus chemotherapy or immunotherapy for first-line treatment of advanced non-small cell lung cancer: a generic protocol. Cochrane Database of Systematic Reviews 2018, Issue 4. Art. No: CD013009. [DOI: 10.1002/14651858.CD013009]

Single or combined immune checkpoint inhibitors compared to first-line platinum-based chemotherapy with or without bevacizumab for 


\section{Tierney 2007}

Tierney JF, Stewart LA, Ghersi D, Burdett S, Sydes MR. Practical methods for incorporating summary time-to-event data into meta-analysis. Trials 2007;8:16. [DOI: 10.1186/1745-6215-8-16]

\section{Tsao 2018}

Tsao MS, Kerr KM, Kockx M, Beasley MB, Borczuk AC, Botling J, et al. PD-L1 Immunohistochemistry Comparability Study in Real-Life Clinical Samples: Results of Blueprint Phase 2 Project. Journal of Thoracic Oncology 2018;13(9):1302-11.

\section{Wallis 2019}

Wallis CJD, Butaney M, Satkunasivam R, Freedland SJ, Patel SP, Hamid O, et al. Association of patient sex with efficacy of immune checkpoint inhibitors and overall survival in advanced cancers: a systematic review and meta-analysis. JAMA Oncology 2019 Apr;5(4):529-36.

\section{CHARACTERISTICS OF STUDIES}

Characteristics of included studies [ordered by study ID]

\section{Yu 2019}

Yu Y, Zeng D, Ou Q, Liu S, Li A, Chen Y et al. Association of survival and immune-related biomarkers with immunotherapy in patients with non-small cell lung cancer: a meta-analysis and individual patient-level analysis. JAMA Network Open 2019 Jul 3;2(7):e196879.

\section{References to other published versions of this review} Ferrara 2019

Ferrara R, Imbimbo M, Paget-Bailly S, Malouf R, Calais F, Agazzi GM, et al. Single or combined immune checkpoint inhibitors compared to first-line chemotherapy with or without bevacizumab for people with advanced non-small cell lung cancer. Cochrane Database of Systematic Reviews 2019, Issue 2. Art. No: CD013257. [DOI: 10.1002/14651858.CD013257]

* Indicates the major publication for the study

Carbone 2017

\section{Study characteristics}

Methods

Study design: randomised open-label parallel controlled, phase 3 trial

Study setting: multicentre trial, 141 study locations

Country: 35 countries: USA, Argentina, Australia, Brazil, Belgium, Canada, Chile, China, Colombia, France, Germany, Greece, Hungary, Ireland, Israel, Italy, Japan, Mexico, the Netherlands, Peru, Poland, Romania, Russian Federetaion, Saudi Arabia, Spain, others

Publication: full-text paper

Study power calculation: yes

Study duration: study start date: March 2014, study completion date: October 2018

Ethical approval: yes, by ethics committee at each centre

Participants Inclusion criteria

- Eastern Cooperative Oncology Group (ECOG) Performance Status (PS) $\leq 1$

- Histologically-confirmed Stage IV, or Recurrent NSCLC with no prior systemic anticancer therapy

- Measurable disease by computed tomography (CT) or magnetic resonance imaging (MRI) per response evaluation criteria in solid tumour version (RECIST) 1.1 criteria

- PD-L1+ (tumour proportional score $\geq 1 \%$ ) on immunohistochemistry testing performed by central laboratory

- Men and women, ages $\geq 18$ years of age

\section{Exclusion criteria}

- Known epidermal growth factor receptor (EGFR) mutations which are sensitive to available targeted inhibitor therapy

- Known anaplastic lymphoma kinase (ALK) translocations

- Untreated central nervous system (CNS) metastases

- Previous malignancies

Single or combined immune checkpoint inhibitors compared to first-line platinum-based chemotherapy with or without bevacizumab for 
Solution for Injection $3 \mathrm{mg} / \mathrm{kg}$ Intravenous every 2 weeks until disease progression, discontinuation due to unacceptable toxicity, withdrawal of consent or study closure

Control: investigator's choice chemotherapy administered in 3-week cycles up to a maximum of 6 cycles of Intravenous injection until disease progression, unacceptable toxicity or completion of the 6 cycles, whichever comes first

\section{Primary outcomes}

- Progression-free survival in participants with PD-L1 expression $\geq 5 \%$ (time frame: from date of randomisation until date of documented tumour progression (assessed up to August 2016, approximately 28 months)

\section{Secondary outcome measures}

- Progression-free survival in all randomised participants (time frame: from date of randomisation until date of documented tumour progression (assessed up to August 2016, approximately 28 months)

- Overall survival in participants with PD-L1 expression $\geq 5 \%$ (time frame: from date of randomisation to date of death (assessed up to August 2016, approximately 28 months)

- Overall survival in all randomised participants (time frame: from date of randomisation to date of death (assessed up to August 2016, approximately 28 months)

- Overall survival in all randomised participants (time frame: from date of randomisation to date of death (assessed up to August 2016, approximately 28 months)

- Objective response rate (ORR) in participants with PD-L1 expression $\geq 5 \%$ (time frame: from date of randomisation until date of documented tumour progression or subsequent anti-cancer therapy, whichever occurs first (assessed up to August 2016, approximately 28 months)

- Duration of response in participants with PD-L1 expression $\geq 5 \%$ (time frame: from date of first confirmed response to date of tumour progression (assessed up to august 2016, approximately 28 months)

- Time to response in participants with PD-L1 expression $\geq 5 \%$ (time frame: from date of randomisation to date of first confirmed response (assessed up to August 2016, approximately 18 months)

- Disease-related symptom improvement rate by week 12 (time frame: from date of randomizations to week 12)

\section{Participants characteristics}

Enrolled $\mathrm{N}=1325$

Randomised N $=541$

Nivolumab group: $\mathrm{N}=271$. Age median, range: 63 (32 to 89 ) years. Age category $\mathrm{n}(\%) \geq 75: 30(11)$. Female $n(\%)$ : 87 (32). Ethnicity/Region n (\%): not reported; ECOG performance $n(\%): 0=85$ (31), $1=183$ (68), $\geq 2:$ 2(1); smoking status $n(\%)$ : former smoker: 186 (69), current smoker: 52 (19), never smoked: 30 (11), unknown = 3 (1). Tumour histological type n (\%): squamous: 66 (24); non-squamous 205 (76); PDL1 expression level $n(\%): \geq 5 \%: 208$ (77), PD-L1 expression level $n(\%) \geq 50 \%: 88$ (32)

Chemotherapy group: $\mathrm{N}=270$. Age median, range: 65 (29 to 87) years. Age category $\mathrm{n}(\%) \geq 75: 32$ (12). Female $n(\%): 122$ (45); Ethnicity/Region $n(\%)$ : not reported; ECOG performance $n(\%): 0=93(34)$, $1=174$ (67), $\geq 2$ : 3(1); smoking status n (\%): former smoker: 182 (67), current smoker: 55 (20), never smoked: 29 (11), unknown: 4 (1). Tumour histological type $n$ (\%): squamous: 64 (24); non-squamous 206 (76); PD-L1 expression level $n(\%): \geq 5 \%: 210$ (78), PD-L1 expression level $n(\%) \geq 50 \%: 126(47)$.

Author's email: david.carbone@osumc.edu

ClinicalTrials.gov Identifier: NCT02041533 
Protocol amendments: the study was amended to include as secondary objective the comparison of overall survival upon nivolumab or investigators' choice chemotherapy among all randomised patients with any PD-L1 positive tumour expression.

\section{Other notes}

Treatment beyond progression allowed in the Nivolumab arm

Cross-over was optional

\section{Risk of bias}

\begin{tabular}{|c|c|c|}
\hline Bias & Authors' judgement & Support for judgement \\
\hline $\begin{array}{l}\text { Random sequence genera- } \\
\text { tion (selection bias) }\end{array}$ & Low risk & $\begin{array}{l}\text { Participants are enrolled using the Interective Voice Response System (IVRS) } \\
\text { and randomised when a randomisation call is made into the IVRS }\end{array}$ \\
\hline $\begin{array}{l}\text { Allocation concealment } \\
\text { (selection bias) }\end{array}$ & Low risk & Central allocation (through a telephone-based randomisation). \\
\hline $\begin{array}{l}\text { Blinding of participants } \\
\text { and personnel (perfor- } \\
\text { mance bias) } \\
\text { All outcomes }\end{array}$ & High risk & $\begin{array}{l}\text { It is an open-label trial, so not blinded for participants and personnel. The ab- } \\
\text { sence of blinding could influence results of primary outcome (progression-free } \\
\text { survival). }\end{array}$ \\
\hline $\begin{array}{l}\text { Blinding of outcome as- } \\
\text { sessment (detection bias) } \\
\text { All outcomes }\end{array}$ & Low risk & $\begin{array}{l}\text { The primary outcome (progression-free survival) is assessed by blinded inde- } \\
\text { pendent central review }\end{array}$ \\
\hline $\begin{array}{l}\text { Incomplete outcome data } \\
\text { (attrition bias) } \\
\text { All outcomes }\end{array}$ & Low risk & $\begin{array}{l}\text { There was a dropout rate of } 22 \% \text { and progression-free survival was mea- } \\
\text { sured on } 423 \text { patients instead of } 541 \text { randomised patients. However, progres- } \\
\text { sion-free survival in all randomised patients (secondary outcome) confirmed } \\
\text { the results observed in } 423 \text { patients limiting the risk of attrition bias. }\end{array}$ \\
\hline $\begin{array}{l}\text { Selective reporting (re- } \\
\text { porting bias) }\end{array}$ & Low risk & $\begin{array}{l}\text { The study protocol is available and the published reports include all expected } \\
\text { outcomes }\end{array}$ \\
\hline Other bias & High risk & $\begin{array}{l}\text { Some baseline differences in patients characteristics (gender, PD-L1 expres- } \\
\text { sion, liver metastases). Some authors declared conflicts of interest related to } \\
\text { the present study or received personal fees from pharmaceutical companies } \\
\text { conducting the trial. }\end{array}$ \\
\hline
\end{tabular}

\section{Study characteristics}

Methods

\begin{abstract}
Study design: randomised parallel controlled trial
Study setting: multicentre trial, 301 study locations

Country: 35 countries: USA, Argentina, Australia, Brazil, Belgium, Canada, Chile, China, Colombia, France, Germany, Greece, Hungary, Ireland, Israel, Italy, Japan, Mexico, the Netherlands, Peru, Poland, Romania, Russian Federetaion, Saudi Arabia, Spain, others
\end{abstract}

Publication: full-text paper

Study power calculation: yes 
Study duration: start date: August 5, 2015, estimated primary completion date November 8, 2020 (Final data collection date for primary outcome measure).

Ethical approval: yes, by ethics committee at each centre

Participants

\section{Inclusion criteria}

- Participants $\geq 18$ years with untreated NSCLC

- Histologically-confirmed stage IV or recurrent NSCLC squamous or non-squamous histology, with no prior systemic anticancer therapy

- Programmed death-ligand 1 (PD -L1) immunohistochemical (IHC) testing, with results, performed by the central laboratory during the screening period

- Eastern Cooperative Oncology Group (ECOG) Performance Status of $\leq 1$

- Measurable disease by CT or MRI per response evaluation criteria in solid tumours version 1.1 (RECIST 1.1) criteria

- Programmed death-ligand 1 (PD -L1) immunohistochemical (IHC) testing, with results, performed by the central laboratory during the screening period

- Eastern Cooperative Oncology Group (ECOG) Performance Status of $\leq 1$

- Measurable disease by CT or MRI per response evaluation criteria in solid tumours version 1.1 (RECIST 1.1) criteria

- Eastern Cooperative Oncology Group (ECOG) Performance Status of $\leq 1$

- Measurable disease by CT or MRI per response evaluation criteria in solid tumours version 1.1 (RECIST 1.1) criteria

\section{Exclusion criteria}

- Untreated central nervous system (CNS) metastases are excluded

- Active, known or suspected autoimmune disease are excluded

- Any positive test for hepatitis B virus or hepatitis C virus or human immunodeficiency virus (HIV) indicating acute or chronic infection

\section{Participants characteristics (subgroup with PD-L1 expression $\geq 1 \%$ )}

Number randomised: 1189

\section{Nivolumab + Ipilimumab}

$\mathrm{N}=396$. Age median, range: 64 (26 to 84 ) years. Age category $\mathrm{n}(\%):<65$ years $=199(50.3) ; \geq 65$ to 75 years: 157 (39.6) years; $\geq 75$ years 40 (10.1); Male $n(\%): 255$ (64.4); ECOG performance $n(\%): 0=135$ (34.1), $1=260$ (65.7); current or former smoker $n(\%)=334$ (84.3); never smoked $n(n \%) 56$ (14.1), unknown = 6 (1.5); Squamous n(\%) = 117 (29.5); Non-squamous 279 (70.5); PD-L1 expression 1-49\%= 191 (48.2), $\geq 50 \%=205$ (51.8), Tumour Mutation Burden (TMB) n (\%): Evaluable =240 (60.6) / $\geq 10 \mathrm{Mut} / \mathrm{Mb}=$ $101(42.1) /<10 \mathrm{Mut} / \mathrm{Mb}=139(57.9)$

\section{Nivolumab}

$\mathrm{N}=396$. Age median, range $=64$ years (27 to 85), Male $n(\%)=272(69) ;$ ECOG performance $0 \mathrm{n}(\%)=142$ (36); squamous $n(\%)=117$ (29.5), non-squamous $n(\%)=279$ (70.5); PD-L1 expression(\%) < 1\% / $\geq 1 \%$ $=0$ (0) / 396 (100); current or former smoker $\mathrm{n}(\%)=342(86)$; never smoked $\mathrm{n}(\%)=50(13)$, unknown $\mathrm{n}$ $(\%)=4$ (1); Tumour Mutation Burden (TMB) n (\%): Evaluable = $228(57.6) / \geq 10 \mathrm{Mut} / \mathrm{mb}=102(45) /<10$ Mut/Mb $=126$ (55).

\section{Chemotherapy}

$\mathrm{N}=397$. Age median, range: 64 (27 to 85$)$ years, Age category $n(\%):<65$ years $=207(52.1) ; 65$ to 75 years: 149 (37.5) years; $\geq 75$ years 41 (10.3). Male $n(\%): 260$ (65.5); ECOG performance $n(\%): 0=134$ $(33.8 \%), 1=259(65.2)$, other or missing = $4(1)$; current or former smoker $n(\%)=340$ (85.6); never smoked 51 (12.8), unknown = 6 (1.5); Squamous n(\%) = 116 (29.2); Non-squamous 281 (70.8); PD-L1 expression level $n(\%) 1 \%$ to $49 \%=205(51.6), \geq 50 \%=192(48.4 \%)$. Tumour Mutation Burden (TMB) $n(\%)$ : Evaluable $=242(61) / \geq 10 \mathrm{Mut} / \mathrm{mb}=112(46.3) /<10 \mathrm{Mut} / \mathrm{Mb}=130(53.7)$ 
Hellmann 2018 (Continued)

Interventions

\section{Randomisation ratio: 1:1:1}

Intervention 1: Nivolumab: 3 mg per kg of body weight plus ipilimumab $1 \mathrm{mg}$ per $\mathrm{kg}$ every 6 weeks

Intervention 2: nivolumab (240 mg every 2 weeks)

Control: Platinum doublet chemotherapy based on tumour histology type (pemetrexed maintenance permitted in eligible people) every 3 weeks for up to four cycles

\section{Outcomes \\ - Primary outcomes (Part 1 of the trial) \\ - Overall survival (OS) (time frame: approximately 48 months ) with nivolumab plus ipilimumab versus chemotherapy in people selected on the basis of the PD-L1 expression level \\ - Progression-free survival (PFS) (Time frame: approximately 40 months) with nivolumab plus ipilimum- ab versus chemotherapy in selected on the basis of TMB using a prespecified cut-off at least 10 muta- tions per megabase}

Notes

\section{Secondary outcomes}

- Progression-free survival (PFS) with nivolumab versus chemotherapy among patients with a TMB of at least 10 mutations per megabase and a PD-L1 expression level of at least $1 \%$

- Overall survival (OS) with nivolumab plus ipilimumab versus platinum doublet chemotherapy among patients with a TMB of at least 10 mutations per megabase.

- Objective response rate (ORR) (time frame: up to 48 months)

- Disease-related symptom improvement as measured by the Lung Cancer Symptom Score (LCSS) (time frame: up to 48 months). Disease-related symptom improvement assessed at each dosing for 6 months, then every 6 weeks while on treatment

Author's email: hellmanm@mskcc.org

ClinicalTrials.gov Identifier: NCT02477826

Protocol amendments: several amendments took place with regard to the planned trial population, biomarkers and primary objectives. Changes occurred after enrolment had been completed but before the locking the database and breaking the treatment codes.

Other notes: treatment beyond progression allowed. Cross-over during the trial was not permitted.

\section{Risk of bias}

\begin{tabular}{lll}
\hline Bias & Authors' judgement & Support for judgement \\
\hline $\begin{array}{l}\text { Random sequence genera- } \\
\text { tion (selection bias) }\end{array}$ & Low risk & $\begin{array}{l}\text { Participants are enrolled using the Interective Voice Response System (IVRS) } \\
\text { and randomised when a randomisation call is made into the IVRS }\end{array}$ \\
\hline $\begin{array}{l}\text { Allocation concealment } \\
\text { (selection bias) }\end{array}$ & Low risk & Central allocation (through a telephone based randomisation). \\
\hline $\begin{array}{l}\text { Blinding of participants } \\
\text { and personnel (perfor- } \\
\text { mance bias) }\end{array}$ & High risk & $\begin{array}{l}\text { Open-label trial design. The absence of blinding could influence results of } \\
\text { some primary outcomes (progression-free survival). }\end{array}$ \\
\hline $\begin{array}{l}\text { Blinding of outcome as- } \\
\text { sessment (detection bias) } \\
\text { All outcomes }\end{array}$ & Low risk & $\begin{array}{l}\text { Primary outcome (progression-free survival) is assessed by blinded indepen- } \\
\text { dent central review, co-primary outcome (overall survival) is not influenced by } \\
\text { blinding of assessment. }\end{array}$ \\
\hline
\end{tabular}


Hellmann 2018 (Continued)

Incomplete outcome data High risk Although number of participants enrolled, randomised and analysed is clear(attrition bias) ly reported, there was a high dropout rate (randomised patients 1739, primary

All outcomes outcome reported in 299 patients)

Selective reporting (re- Low risk Protocol was available and all outcomes were reported
porting bias)

Other bias High risk

Although tumour mutational burden analysis was performed before database lock and breaking of coded treatments, this amendment substantially affect the primary and secondary endpoints of the study. Some authors declared conflicts of interest related to the present study or received personal fees from pharmaceutical companies conducting the trial.

\section{Study characteristics}

Study design: randomised open-label parallel controlled, phase 3 trial
Study setting: multicentre trial, 191 planned study locations
Country: 19 countries USA, Brazil, China, France, UK, Germany, Greece, Hungry, Italy, Poland, Romania,
Russia, Serbia, Spain, Ukraine,Japan, Korea, Thailand,Turkey)

Publication: abstract

\section{Inclusion criteria}

- Histologically or cytologically confirmed, Stage IV non-squamous or squamous NSCLC

- No prior treatment for Stage IV non-squamous or squamous NSCLC. Participant known to have a sensitising mutation in the epidermal growth factor receptor (EGFR) gene or an anaplastic lymphoma kinase (ALK) fusion oncogene are excluded from the study

- Tumour PD-L1 expression as determined by immunohistochemistry (IHC) assay of archival tumour tissue or tissue obtained at screening. Only people with PD-L1 expression $\geq 1 \%$ on tumour cells (TC) or immune cells (IC) were included.

- Eastern Cooperative Oncology Group (ECOG) performance status 0 to 1

- Measurable disease as defined by Response Evaluation Criteria in Solid Tumors (RECIST v1.1)

- Adequate haematological and end-organ function

\section{Exclusion criteria}

- Known sensitising mutation in the EGFR gene or ALK fusion oncogene

- Active or untreated central nervous system (CNS) metastases as determined by Computed Tomography (CT) or magnetic resonance imaging (MRI) evaluation

- Malignancies other than NSCLC within 5 years prior to randomisation, with the exception of those with a negligible risk of metastasis or death treated with expected curative outcome

- Pregnant or lactating women

- History of autoimmune disease

- History of idiopathic pulmonary fibrosis, organising pneumonia, drug induced pneumonitis, idiopathic pneumonitis, or evidence of active pneumonitis on screening chest CT scan. History of radiation pneumonitis in the radiation field (fibrosis) is permitted

- Positive test for Human Immunodeficiency Virus (HIV)

- Active hepatitis B or hepatitis C 
Herbst 2020 (Continued)

- Prior treatment with cluster of differentiation (CD) 137 agonists or immune checkpoint blockade therapies, anti PD1, and anti-PD-L1 therapeutic antibody

- Severe infection within 4 weeks prior to randomisation

- Significant history of cardiovascular disease

\section{Participants characteristics}

Number randomised: 554

\section{Atezolizumab group}

$\mathrm{N}=277$. Age < 65 years, $\mathrm{n}(\%): 143$, (51.6). Male sex $n(\%): 196$ (70.8); ECOG performance $\mathrm{n}(\%): 0: 97$ (35); never smoked 37 (13.4. Histology: non-squamous n (\%) = $192(69.3)$

\section{Chemotherapy group:}

$N=277$. Age < 65 years $n(\%): 134$, (48.4). Male sex $n(\%): 193$ (69.7); ECOG performance $n(\%): 0: 102$ (36.8); never smoked 35 (12.6). Histology: non-squamous n (\%) = 193 (69.7)

Interventions

Intervention: atezolizumab, other names: MPDL3280A, R05541267

Atezolizumab 1200 milligram (mg), intravenous infusion every 21 days until loss of clinical benefit (as assessed by the investigator), unacceptable toxicity, or death (maximum up to approximately 58 months).

\section{Comparators:}

(Carboplatin/Cisplatin) + (Pemetrexed/ Gemcitabine)

Participants with non-squamous NSCLC will receive chemotherapy with pemetrexed in combination with either cisplatin or carboplatin (per investigator discretion) on Day 1 of each 21-day cycle for 4 or 6 cycles as per local standard of care, followed by maintenance therapy with pemetrexed alone as per local standard of care until disease progression (per RECIST v1.1), unacceptable toxicity, or death (maximum up to approximately 58 months). Participants with squamous NSCLC will receive chemotherapy with gemcitabine on Days 1 and 8 of each 21-day cycle in combination with either cisplatin or carboplatin on Day 1 of each 21-day cycle for 4 or 6 cycles as per local standard of care, followed by best supportive care as per local standard of care until disease progression, unacceptable toxicity, or death (maximum up to approximately 58 months).

\section{Primary outcome measures}

Overall survival (OS): time frame: from randomisation to death from any cause (maximum up to approximately 58 months) in PD-L1 positive (tumour cell (TC) or immune cell score (IC) $\geq 1 \%$ )

The primary endpoint was tested hierarchically in the following subgroups: PD-L1 $\geq 50 \%$ on TC or IC (TC3 or IC3); PD-L1 $\geq 5 \%$ on TC or IC (TC $2 / 3$ or IC $2 / 3$ ); and PD-L $1 \geq 1 \%$ on TC or IC (TC $1 / 2 / 3$ or IC $1 / 2 / 3$ ).

\section{Secondary outcome measures}

- :Progression-free Survival (PFS): time frame: from randomisation to the first occurrence of disease progression or death from any cause, whichever occurs first (up to approximately 58 months)

- Percentage of participants with objective response (ORR): time frame: every 6 weeks for 48 weeks following day 1 , thereafter every 9 weeks after completion of the week 48 tumour assessment, regardless of treatment delays, until radiographic disease progression (maximum up to approximately 58 months) ]

- Duration of Response (DOR):time frame: from the first occurrence of a complete response (CR) or partial response (PR), whichever occurs first, until the first date that progressive disease or death is documented, whichever occurs first (up to approximately 58 months)

- Percentage of participants who are alive at 1 and 2 Years, time frame: 1 and 2 years

- Time to deterioration (TTD) in patient-reported Lung Cancer Symptoms Score as assessed by the Symptoms in Lung Cancer (SILC) Scale Symptom Score (time frame: Baseline up to approximately 58 months) 
Herbst 2020 (Continued)

- Change From Baseline in patient-reported Lung Cancer Symptoms Score as Assessed by the SILC Scale Symptom Score (time frame: baseline up to approximately 58 months)

- TTD as assessed using European Organization for the Research and Treatment of Cancer (EORTC) Quality of Life Questionnaire-Core (EORTC QLQ-C30) (time frame: baseline up to approximately 58 months)

- TTD as assessed using EORTC QLQ Supplementary Lung Cancer Module (EORTC QLQ-LC13) (time frame: baseline up to approximately 58 months)

- OS in participants with PD-L1 expression (time frame: from randomisation to death from any cause (maximum up to approximately 58 months)

- Investigator-assessed PFS in participants with PD-L1 expression according to RECIST V1.1 (time frame: from randomisation to the first occurrence of disease progression or death from any cause, whichever occurs first (up to approximately 58 months)

- OS in participants with blood Tumour Mutational Burden (bTMB) (time frame: from randomisation to death from any cause (maximum up to approximately 58 months)

- Investigator-assessed PFS in participants with bTMB according to RECIST v1.1 (time frame: from randomisation to the first occurrence of disease progression or death from any cause, whichever occurs first (up to approximately 58 months)

Notes Sponsorship source: Roche

ClinicalTrials.gov Identifier: NCT02409342

Study protocol: not published

Other notes: Cross-over during the trial was not permitted

\section{Risk of bias}

\begin{tabular}{|c|c|c|}
\hline Bias & Authors' judgement & Support for judgement \\
\hline $\begin{array}{l}\text { Random sequence genera- } \\
\text { tion (selection bias) }\end{array}$ & Low risk & Randomisation number was obtained by a web-based response system \\
\hline $\begin{array}{l}\text { Allocation concealment } \\
\text { (selection bias) }\end{array}$ & Low risk & $\begin{array}{l}\text { Central allocation was performed. Study sites obtained participants randomi- } \\
\text { sation number and treatment assignment from interactive voice or web-based } \\
\text { response system }\end{array}$ \\
\hline $\begin{array}{l}\text { Blinding of participants } \\
\text { and personnel (perfor- } \\
\text { mance bias) } \\
\text { All outcomes }\end{array}$ & Low risk & $\begin{array}{l}\text { No blinding it is an open-label study design. However, the absence of blinding } \\
\text { unlikely influenced results of the primary outcome (OS). }\end{array}$ \\
\hline $\begin{array}{l}\text { Blinding of outcome as- } \\
\text { sessment (detection bias) } \\
\text { All outcomes }\end{array}$ & Low risk & $\begin{array}{l}\text { Primary endpoint (overall survival) was not influenced by the absence of blind- } \\
\text { ing assessment }\end{array}$ \\
\hline $\begin{array}{l}\text { Incomplete outcome data } \\
\text { (attrition bias) } \\
\text { All outcomes }\end{array}$ & Low risk & Outcome reported as intention to treat (ITT) population. No drop outs \\
\hline $\begin{array}{l}\text { Selective reporting (re- } \\
\text { porting bias) }\end{array}$ & Low risk & Protocol was available and all outcomes were reported. \\
\hline Other bias & Unclear risk & $\begin{array}{l}\text { Some authors declared conflicts of interest related to the present study or re- } \\
\text { ceived personal fees from pharmaceutical companies conducting the trial. }\end{array}$ \\
\hline
\end{tabular}

Single or combined immune checkpoint inhibitors compared to first-line platinum-based chemotherapy with or without bevacizumab for 
Mok 2019

\section{Study characteristics}

Methods

Study design: randomised open-label parallel controlled, phase 3 trial

Study setting: multicentre trial, 213 study locations

Country: 32 countries: USA, Argentina, Australia, Brazil, Belgium, Canada, Chile, China, Colombia, France, Germany, Greece, Hungary, Ireland, Israel, Italy, Japan, Mexico, the Netherlands, Peru, Poland, Romania, Russian Federetaion, Saudi Arabia, Spain, others

Publication: full-text paper

Study power calculation: yes

Study duration: December 2014 to March 2017

Ethical approval: yes, by ethics committee at each centre

Participants

Inclusion criteria

- Participants $\geq 18$ years with untreated NSCLC

- Histologically- or cytologically-confirmed diagnosis of advanced or metastatic NSCLC

- PD-L1 positive tumour

- Measureable disease based on Response Evaluation Criteria in Solid Tumors (RECIST) 1.1

- Life expectancy of at least 3 months

- No prior systemic chemotherapy for the treatment of the participant's advanced or metastatic disease (treatment with chemotherapy and/or radiation as part of neoadjuvant/adjuvant therapy is allowed as long as completed at least 6 months prior to diagnosis of advanced or metastatic disease)

- Eastern Cooperative Oncology Group (ECOG) Performance Status of 0 or 1

- Adequate organ function

- No prior malignancy, with the exception of basal cell carcinoma of the skin, superficial bladder cancer, squamous cell carcinoma of the skin, or in situ cancer, or has undergone potentially curative therapy with no evidence of that disease recurrence for 5 years since initiation of that therapy

- Submission of formalin-fixed diagnostic tumour tissue (in the case of participants having received adjuvant systemic therapy, the tissue should be taken after completion of this therapy)

- Female participants of childbearing potential must have a negative urine or serum pregnancy test and must be willing to use two adequate barrier methods of contraception or a barrier method plus a hormonal method starting with the screening visit through 120 days after the last dose of pembrolizumab or 180 days after the last dose of chemotherapeutic agents used in the study

- Male participants with a female partner(s) of child-bearing potential must be willing to use two adequate barrier methods of contraception from screening through 120 days after the last dose of pembrolizumab or 180 days after the last dose of chemotherapeutic agents used in the study

\section{Exclusion criteria}

- Epidermal growth factor receptor (EGFR)-sensitising mutation and/or is echinoderm microtubule-associated protein-like 4 (EML4) gene/anaplastic lymphoma kinase (ALK) gene fusion positive

- Currently participating or has participated in a study of an investigational agent or using an investigational device within 4 weeks of the first dose of study therapy

- No tumour specimen evaluable for PD-L1 expression by the central study laboratory

- Squamous histology and received carboplatin in combination with paclitaxel in the adjuvant setting

- Is receiving systemic steroid therapy $\leq 3$ days prior to the first dose of study therapy or receiving any other form of immunosuppressive medication with the exception of daily steroid replacement therapy

- The NSCLC can be treated with curative intent with either surgical resection and/or chemoradiation

- Expected to require any other form of systemic or localised antineoplastic therapy while on study

- Any prior systemic cytotoxic chemotherapy, biological therapy or major surgery within 3 weeks of the first dose of study therapy; received lung radiation therapy $>30$ Gy within 6 months of the first dose of study therapy 
- Prior therapy with an anti-PD-1, anti-PD-L1, anti-PD-L2, anti-CD137, or anti-cytotoxic T-lymphocyte-associated antigen-4 (CTLA-4) antibody (including ipilimumab or any other antibody or drug specifically targeting T-cell co-stimulation or checkpoint pathways)

- Known central nervous system metastases and/or carcinomatous meningitis

- Active autoimmune disease that has required systemic treatment in the past 2 years

- Had allogeneic tissue/solid organ transplantation

- Interstitial lung disease or history of pneumonitis that has required oral or IV steroids

- Has received or will receive a live vaccine within 30 days prior to the first study therapy (seasonal flu vaccines that do not contain live vaccine are permitted)

- Active infection requiring intravenous systemic therapy

- Known history of human immunodeficiency virus (HIV)

- Known active Hepatitis B or C

- Regular user (including "recreational use") of any illicit drugs or had a recent history (within the last year) of substance abuse (including alcohol)

- Pregnant, breastfeeding, or expecting to conceive or father children within the projected duration of the study

\section{Participants characteristics}

Number screened $=3428$

Number with PD-L1 expression $=3019,1978$ had PD-L1 expression $\geq 1 \%$

Number included in Intertion to treat (ITT): 1274 (637 in each arm)

Pembrolizumab group (Tumour proportional score PD-L1 => 1\%)

$N=637$. Age median, (range): 63, (56 to 69 years). Age category n (\%): <65: 359 (56); Male sex n (\%): 450 (71). Ethnicity/Region n (\%): East Asia: 185 (29), Europe :149 (23); Latin America: 78 (19); Other 111 (27); ECOG performance $n(\%): 0: 198$ (31), 1: 439 (69); current or former smoker never smoked 125 (20), 370 (58), 142 (22). Histology: squamous $n(\%)=243$ (38); non-squamous 394 (62); brain metastases $n(\%)$ : 35 (5); Disease status n (\%): locally advanced: 76 (12), metastatic 561 (88); PD-L1 tumour proportional score n (\%): $1 \%$ to $19 \%: 224$ (35), 20\% to 49\%: 114 (18); $\geq 50 \%: 299$ (47)

\section{Chemotherapy group}

$N=637$.. Age median, (range): 63, (57 to 69 years). Age category n (\%): <65: 348 (55); Male sex n (\%): 452 (71). Ethnicity/Region n (\%): East Asia: 185 (29), Europe :137 (22); Latin America: 133 (21); Other 182 (29); ECOG performance n (\%): 0: 192 (30), 1: 445 (70); current or former smoker never smoked 146 (23), 351 (55), 140 (22), Histology: squamous n (\%) = 249 (39); non-squamous 388 (61); brain metastases $n$ (\%): 35 (5); Disease status n (\%): locally advanced: 84 (13), metastatic 553 (87); PD-L1 tumour proportional score n (\%): \% to 19\%: 232 (36), 20\% to 49\%: 105 (16); $\geq 50 \%: 300$ (47)

Interventions

Intervention: pembrolizumab

200 mg intravenous (IV) on Day 1 of every 21-day cycle (every 3 weeks, or Q3W) for up to 35 treatments.

Control: standard of care (SOC) platinum-based chemotherapy (carboplatin + paclitaxel or carboplatin + pemetrexed for 4 to 621 -day cycles)

Outcomes

\section{Primary outcomes}

- Overall survival (OS) in participants with a tumour proportion score (TPS) of $\geq 50 \%$ (up to approximately 38 months)

- Overall survival (OS) in participants with a tumour proportion score (TPS) of $\geq 20 \%$ (up to approximately 38 months)

- Overall survival (OS) in participants with a tumour proportion score (TPS) of $\geq 1 \%$ (up to approximately 38 months)

\section{Secondary outcomes}

Single or combined immune checkpoint inhibitors compared to first-line platinum-based chemotherapy with or without bevacizumab for 
- Progression-free survival (PFS) participants with a tumour proportion score (TPS) of $\geq 50 \%$ (up to approximately 38 months)

- Progression-free survival (PFS) in participants with a tumour proportion score (TPS) of $\geq 20 \%$ (up to approximately 38 months)

- Progression-free survival (PFS) in participants with a tumour proportion score (TPS) of $\geq 1 \%$ (up to 38 months)

- Number of participants who experienced at least one adverse event (AE) (time frame: through database cut-off date of 26-Feb-2018 (up to approximately 38 months)

- Number of participants who discontinued study treatment due to an adverse event (AE) (time frame: through database cut-off date of 26-Feb-2018 (up to approximately 38 months)

Sponsorship source: Merck Sharp \& Dohme Corp
ClinicalTrials.gov Identifier: NCT02220894
Study protocol: published
Protocol amendments: primary endpoint was changed during the trial ( and overall survival in pa-
tients with PD-L1 $\geq 1 \%$ on tumour cells was included as primary endpoint). In a second amendment
a new cut-off for PD-L1 expression on tumour cells (TPS 20\%) was tested and primary and secondary
endpoints were amended to include OS, PFS and objective response, respectively, in people with PD-L1
TPS $\geq 50 \%, \geq 20 \%$ and $\geq 1 \%$.

\section{Risk of bias}

\begin{tabular}{lll}
\hline Bias & Authors' judgement & Support for judgement \\
\hline $\begin{array}{l}\text { Random sequence genera- } \\
\text { tion (selection bias) }\end{array}$ & Low risk & $\begin{array}{l}\text { Randomisation was computer generated, accessed via an interactive voice-re- } \\
\text { sponse and integrated web-response system, and stratified by region of enrol- } \\
\text { ment (east Asia vs rest of world) }\end{array}$ \\
\hline
\end{tabular}

\begin{tabular}{lll}
\hline $\begin{array}{l}\text { Allocation concealment } \\
\text { (selection bias) }\end{array}$ & Low risk & $\begin{array}{l}\text { Enrolled participants were randomly assigned 1:1 in blocks of four per stratum } \\
\text { (central allocation) }\end{array}$ \\
\hline $\begin{array}{l}\text { Blinding of participants } \\
\text { and personnel (perfor- }\end{array}$ & Low risk & $\begin{array}{l}\text { The study was open-label due to quote: "differences in infusion durations, ad- } \\
\text { mance bias) }\end{array}$ \\
$\begin{array}{l}\text { mill outcomestration schedules and requirement for pre medications". However, the } \\
\text { absence of blinding unlikely influenced results of primary outcome (OS) }\end{array}$
\end{tabular}

\begin{tabular}{lll}
\hline $\begin{array}{l}\text { Blinding of outcome as- } \\
\text { sessment (detection bias) } \\
\text { All outcomes }\end{array}$ & Low risk & Central radiological reviewers were unaware of treatment assignments \\
\hline $\begin{array}{l}\text { Incomplete outcome data } \\
\text { (attrition bias) }\end{array}$ & Low risk & $\begin{array}{l}\text { Number of participants eligible of inclusion in the trial, number included in } \\
\text { each arm and the number of participants who discontinued all clearly docu- } \\
\text { mented. Reasons for dropout were also documented. Dropout regarded only } 1\end{array}$ \\
& & patient. Outcomes were measured on randomised patients.
\end{tabular}

\begin{tabular}{|c|c|c|}
\hline $\begin{array}{l}\text { Selective reporting (re- } \\
\text { porting bias) }\end{array}$ & Low risk & Protocol was available and all outcomes were reported \\
\hline Other bias & High risk & $\begin{array}{l}\text { Protocol amended changing the primary and secondary endpoints and a new } \\
\text { cut off point (PD-L1 tumour proportional score } 20 \% \text { ) was included. Some au- } \\
\text { thors declared conflicts of interest related to the present study or received per- } \\
\text { sonal fees from pharmaceutical companies conducting the trial. }\end{array}$ \\
\hline
\end{tabular}


Reck 2016

\section{Study characteristics}

Methods

Study design: randomised open-label parallel controlled, phase 3 trial

Study setting: multicentre trial, 102 study locations

Country: 16 countries,

Publication: full-text paper

Study power calculation: yes

Study duration: September 2014 to October 2015

Median follow-up period: 25.2 months (data cut-off in July 2017)

Ethical approval: yes, by ethics committee at each centre

- Histological or cytological diagnosis of Stage IV NSCLC lacking epidermal growth factor receptor (EGFR)-sensitising mutation and/or anaplastic lymphoma kinase (ALK) translocation, and received no prior systemic chemotherapy treatment for their metastatic NSCLC

- At least one radiographically-measurable lesion per RECIST 1.1

- Life expectancy of at least 3 months

- Performance status of 0 or 1 on the Eastern Cooperative Oncology Group (ECOG) Performance Status

- Adequate organ function

- No history of prior malignancy, with the exception of basal cell carcinoma of the skin, superficial bladder cancer, squamous cell carcinoma of the skin, or in situ cervical cancer, or has undergone potentially curative therapy with no evidence of that disease recurrence for 5 years since initiation of that therapy

- Provided newly obtained formalin fixed tumour tissue from a biopsy of a tumour at the time of or after the diagnosis of metastatic disease has been made and from a site not previously irradiated

- PD-L1 strong expressing tumour as determined by immunohistochemistry (IHC) at a central laboratory

- Female participants must have a negative pregnancy test at screening if of childbearing potential or be of non-childbearing potential

- Female participants of childbearing potential and male partners with female partners of childbearing potential must agree to use 2 adequate barrier methods of contraception during the study and for 120 days after last dose of study drug and up to 180 days after last dose of chemotherapy

\section{Exclusion criteria}

- EGFR sensitising mutation and/or ALK translocation

- Has received systemic therapy for the treatment of their stage IV NSCLC. Completion of treatment with chemotherapy and/or radiation as part of neoadjuvant/adjuvant therapy is allowed as long as therapy was completed at least 6 months prior to the diagnosis of metastatic disease.

- Currently participating or has participated in a study of an investigational agent or using an investigational device within 30 days of first dose of study drug

- Tumour specimen is not available for PD-L1 expression by the central laboratory

- Receiving systemic steroid therapy $<=3$ days prior to first dose of study drug or receiving any other form of immunosuppressive medication

- Expected to require any other form of systemic or localised antineoplastic therapy during the study

- Received prior systemic cytotoxic chemotherapy, biological therapy, major surgery within 3 weeks of first dose of study drug; received thoracic radiation therapy of $>30$ Gray (Gy) within 6 months of first dose of study drug

- Received prior therapy with an anti-programmed cell death protein 1 (anti-PD-1), anti-PD-L1, anti-programmed cell death-ligand 2 (anti-PD-L2), anti-CD137 (4-1BB ligand, a member of the Tumor Necrosis Factor Receptor (TNFR) family), or anti-Cytotoxic T-lymphocyte-associated antigen-4 (anti-CTLA-4)

Single or combined immune checkpoint inhibitors compared to first-line platinum-based chemotherapy with or without bevacizumab for 
Reck 2016 (Continued)

antibody (including ipilimumab or any other antibody or drug specifically targeting T-cell co-stimulation or checkpoint pathways)

- Has untreated central nervous system (CNS) metastases and/or carcinomatous meningitis

- Active autoimmune disease that has required systemic treatment in past 2 years

- Allogenic tissue/solid organ transplant

- Interstitial lung disease or pneumonitis that has required oral or IV steroids

- Received or will receive a live vaccine within 30 days prior to first dose of study drug

- Active infection requiring IV systemic therapy

- Known history of human immunodeficiency virus (HIV)

- Known active tuberculosis, or hepatitis B or C

- Known psychiatric or substance abuse disorders that would interfere with cooperation with the requirements of the study

- Is, at the time of signing informed consent, a regular user (including "recreational use") of any illicit drugs or had a recent history (within the last year) of substance abuse (including alcohol)

- Pregnant or breastfeeding, or expecting to conceive or father children during the study and through 120 days after last dose of pembrolizumab or 180 days after last dose of SOC chemotherapy

- Immediate family member who is investigational site or sponsor staff directly involved with this study

\section{Participants characteristics}

Number eligible: 1934

Number randomised: 305

\section{Pembrolizumab group}

$\mathrm{N}=154$. Age median, (range): 64.5, (33 to 90 years). Male sex $\mathrm{n}(\%): 92$ (59.7), Ethnicity/Region $\mathrm{n}(\%)$ : East Asia: 21 (13.6), Non-East Asia: 133 (86.4); ECOG performance n (\%): 0: 54 (35.1), 1: 99 (64.3); current or former smoker never smoked 34 (22.1), 115 (74.7), 5 (3.2). Histology: squamous n (\%) = 29 (18.8); nonsquamous 125 (81.2); brain metastases $n(\%): 18$ (11.7)

\section{Chemotherapy group}

$\mathrm{N}=151$. Age median, (range): 66, (38 to 85 years). Male sex $\mathrm{n}(\%): 95$ (62.9). Ethnicity/Region $\mathrm{n}(\%)$ : East Asia: 19 (12.6), Non-East Asia: 132 (87.4); ECOG performance n (\%): 0: 53 (35.1), 1: 98 (64.9); current or former smoker never smoked 31 (20.5), 101 (66.9), 19 (12.6). Histology: squamous n(\%) = 27 (17.9); nonsquamous 124 (82.1); brain metastases $n$ (\%): 10 (6.6)

Interventions

Randomisation ratio:1:1

Intervention group: pembrolizumab 200 mg every three weeks (35 cycles)

Control group: platinum-doublet chemotherapy 4 to 6 cycles

Outcomes Primary outcome

- Progression-free survival (PFS) rate at month 6 (time frame: month 6)

\section{Secondary outcomes}

- Overall survival (OS) rate at month 6 (time frame: month 6)

- Objective response rate (ORR) (time frame: through data cut-off data of 09 May 2016 (up to approximately 1.6 years)

- Quality of life using EORCT Quality of life questionnaire Lung Cancer and the European Quality of Life 5 dimensions

Cross-over phase: this is only applicable for participants randomised to receive SOC. Eligible participants were treated with pembrolizumab for the remainder of the study or until disease progression,

Single or combined immune checkpoint inhibitors compared to first-line platinum-based chemotherapy with or without bevacizumab for 
Reck 2016 (Continued)

unacceptable AEs, intercurrent illness that prevents further administration of treatment, investigator's decision to withdraw the participant, noncompliance with study treatment or procedures requirements, the participant receives 35 treatments of study treatment (pembrolizumab arm only), or administrative reasons.

\section{Risk of bias}

\begin{tabular}{|c|c|c|}
\hline Bias & Authors' judgement & Support for judgement \\
\hline $\begin{array}{l}\text { Random sequence genera- } \\
\text { tion (selection bias) }\end{array}$ & Low risk & $\begin{array}{l}\text { Random sequence generated using Interactive Voice Response System (IVRS) } \\
\text { and integrated web response system (IWRS) }\end{array}$ \\
\hline $\begin{array}{l}\text { Allocation concealment } \\
\text { (selection bias) }\end{array}$ & Low risk & Central allocation \\
\hline $\begin{array}{l}\text { Blinding of participants } \\
\text { and personnel (perfor- } \\
\text { mance bias) } \\
\text { All outcomes }\end{array}$ & High risk & $\begin{array}{l}\text { Open-label design trial. The absence of blinding could influence results of pri- } \\
\text { mary outcome (progression-free survival) }\end{array}$ \\
\hline $\begin{array}{l}\text { Blinding of outcome as- } \\
\text { sessment (detection bias) } \\
\text { All outcomes }\end{array}$ & Low risk & Assessment was done by blinded independent central radiologic review \\
\hline $\begin{array}{l}\text { Incomplete outcome data } \\
\text { (attrition bias) } \\
\text { All outcomes }\end{array}$ & Low risk & $\begin{array}{l}\text { Primary and secondary endpoints were reported on the intention to treat pop- } \\
\text { ulation }\end{array}$ \\
\hline $\begin{array}{l}\text { Selective reporting (re- } \\
\text { porting bias) }\end{array}$ & Low risk & Outcomes reported for all groups \\
\hline Other bias & Unclear risk & $\begin{array}{l}\text { Some authors declared conflicts of interest related to the present study or re- } \\
\text { ceived personal fees from pharmaceutical companies conducting the trial }\end{array}$ \\
\hline
\end{tabular}

Rizvi 2020

\section{Study characteristics}

Study design: randomised open-label parallel controlled, phase 3 trial
Study setting: multicentre trial, 167 study locations
Country: 17 countries, USA, Canada, Europe, Russia, Australia and parts of Asia, including Japan, Ko-
rea, Thailand, Taiwan and Vietnam.

Publication: Abstract

Inclusion criteria
- Aged at least 18 years
- Documented evidence of Stage IV NSCLC
- No sensitising EGFR mutation or ALK rearrangement
- No prior chemotherapy or any other systemic therapy for recurrent/metastatic NSCLC

\section{Exclusion criteria}

Single or combined immune checkpoint inhibitors compared to first-line platinum-based chemotherapy with or without bevacizumab for 
- Mixed small-cell lung cancer and NSCLC histology, sarcomatoid variant

- Brain metastases or spinal cord compression unless asymptomatic, treated and stable (not requiring steroids)

- Prior exposure to Immunomodulatory therapy (IMT), including, but not limited to, other anti-cytotoxic T-lymphocyte-associated antigen 4 (CTLA-4), anti-programmed cell death1 (PD-1), anti-programmed cell death ligand 1 (PD-L1), or anti PD-L2 antibodies, excluding therapeutic anticancer vaccines

- Active or prior documented autoimmune or inflammatory disorders (including inflammatory bowel disease (e.g., colitis or Crohn's disease)

\section{Participants Characterisctics}

Number randomised: 1118

\section{Durvalumab group (PD-L1 TPS $\geq 25 \%$ )}

$\mathrm{N}=163$. Age median, (range): 64, (32 to 84 years). Male sex $n$ (\%): 113 (69.3); ECOG performance $\mathrm{n}(\%)$ : 0: 57 (35); current/former/never smoked 47 (28.8), 92 (56.4), 24 (14.7). Histology: squamous n (\%) = 52 (31.9)

\section{Durvalumab plus tremelimumab group (PD-L1 TPS $\geq 25 \%$ )}

$\mathrm{N}=163$. Age median, (range): 65, (34 to 87 years), Male sex $n$ (\%): 118 (72.4); ECOG performance $\mathrm{n}(\%)$ : 0: 65 (39.9); current/former/never smoked 42 (25.8), 96 (58.9), 25 (15.3). Histology: squamous n (\%) = 53 (32.5)

\section{Chemotherapy group (PD-L1 TPS $\geq 25 \%$ )}

$\mathrm{N}=162$. Age median, (range): 64.5, (35 to 85 years). Male sex $\mathrm{n}(\%)$ : 106 (65.4); ECOG performance $\mathrm{n}(\%)$ : 0: 70 (43.2); current/former/never smoked 39 (24.1), 102 (63), 22 (13). Histology: squamous n(\%) = 52 (32.1)

Intervention1: PD-L1 monoclonal antibody monotherapy (durvalumab); (20 mg/kg i.v. q4w)

Intervention 2 : durvalumab + tremelimumab combination therapy, (D: 20 mg/kg i.v. q4w; T: 1 mg/kg i.v. q4w (up to 4 doses)

Control: chemotherapy (intended up to 6 cycles; pemetrexed maintenance permitted in eligible people) until disease progression

- Progression-free survival (PFS) and Overall survival (OS) in patients with durvalumab and tremelimumab combination compared to chemotherapy in people with PD-L1 $\geq 25 \%$ (time frame: 3 years)

- Overall survival (OS) with durvalumab compared to chemotherapy in people with PD-L1 $\geq 25 \%$ (time frame: 3 years)

\section{Secondary outcomes}

- Objective response rate (ORR) or progression-free survival (PFS) with durvalumab compared to chemotherapy in people with PD-L1 $\geq 25 \%$ (time frame: 3 years)

- Objective response rate (ORR) with durvalumab and tremelimumab compared to chemotherapy (in people with PD-L1 $\geq 25 \%, \geq 1 \%$ and in the overall population) (time frame: 3 years)

- The safety and tolerability profile of durvalumab + tremelimumab combination therapy and durvalumab monotherapy compared to $\mathrm{SoC}$ will be determined using vital signs, laboratory data, electrocardiograms (ECGs), and physical examination (time frame: 3 years) 
Rizvi 2020 (Continued)

Other notes: Cross-over during the trial was not permitted

\section{Risk of bias}

\begin{tabular}{lll}
\hline Bias & Authors' judgement & Support for judgement \\
\hline $\begin{array}{l}\text { Random sequence genera- } \\
\text { tion (selection bias) }\end{array}$ & Low risk & $\begin{array}{l}\text { Using the Interactive Voice Response System (IVRS) and integrated web re- } \\
\text { sponse system (IWRS) }\end{array}$ \\
\hline $\begin{array}{l}\text { Allocation concealment } \\
\text { (selection bias) }\end{array}$ & Low risk & $\begin{array}{l}\text { A blocked randomisation is used and all the centres use the same randomisa- } \\
\text { tion list in order to minimize any imbalance in the number of patients assigned } \\
\text { to each treatment group. }\end{array}$
\end{tabular}

$\begin{array}{ll}\begin{array}{l}\text { Blinding of participants } \\ \text { and personnel (perfor- }\end{array} & \text { High risk } \\ \text { some primary outcomes (progression-free survival). }\end{array}$

mance bias)

All outcomes

\begin{tabular}{|c|c|c|}
\hline $\begin{array}{l}\text { Blinding of outcome as- } \\
\text { sessment (detection bias) } \\
\text { All outcomes }\end{array}$ & Low risk & $\begin{array}{l}\text { The primary endpoint is progression-free survival by blinded independent cen- } \\
\text { tral review assessment }\end{array}$ \\
\hline
\end{tabular}

\begin{tabular}{lll}
\hline $\begin{array}{l}\text { Incomplete outcome data } \\
\text { (attrition bias) } \\
\text { All outcomes }\end{array}$ & High risk & $\begin{array}{l}\text { The primary analysis population for the study was amended to include on- } \\
\text { ly people with PD-L1 expression } \geq 25 \%, \text { therefore only } 488 \text { (44\%) of } 1118 \text { ran- } \\
\text { domised people were included in the analysis of the trial primary outcome }\end{array}$ \\
\hline $\begin{array}{l}\text { Selective reporting (re- } \\
\text { porting bias) }\end{array}$ & Low risk & Outcomes reported for all groups \\
\hline Other bias & Unclear risk & $\begin{array}{l}\text { Some authors declared conflicts of interest related to the present study or re- } \\
\text { ceived personal fees from pharmaceutical companies conducting the trial. }\end{array}$ \\
\hline
\end{tabular}

\section{Study characteristics}

Methods

Study design: randomised open-label parallel controlled, phase 3 trial

Study setting: multicentre trial, 188 study locations

Country: 26 countries,

Publication: second interim analysis at a conference presentation

Study power calculation: not reported

Study duration: May 2017 - ongoing

Median follow-up period: $\mathbf{1 0 . 8}$ months (data cut-off in March 2020)

Ethical approval: yes, by ethics committee at each centre

710 participants, 563 with PD-L1=> 50\%
Inclusion criteria
- Patients with histologically- or cytologically-documented squamous or non squamous NSCLC with
stage IIIB or stage IIIC disease who are not candidates for treatment with definitive concurrent


chemoradiation or patients with stage IV disease who received no prior systemic treatment for recurrent or metastatic NSCLC

- Archival or newly obtained formalin-fixed tumour tissue from a metastatic/recurrent site, which has not previously been irradiated

- Tumour cells expressing PD L1 above a specific percentage of tumour cells by IHC performed by the central laboratory

- At least 1 radiographically-measurable lesion per RECIST 1.1

- ECOG performance status of $\leq 1$

- Anticipated life expectancy of at least 3 months

- Adequate organ and bone marrow function

\section{Exclusion Criteria}

- Patients that have never smoked, defined as smoking $<100$ cigarettes in a lifetime

- Active or untreated brain metastases or spinal cord compression

- Patients with tumours tested positive for EGFR gene mutations, ALK gene translocations, or ROS1 fusions

- Encephalitis, meningitis, or uncontrolled seizures in the year prior to randomisation

- History of interstitial lung disease (e.g. idiopathic pulmonary fibrosis, organizing pneumonia) or active, noninfectious pneumonitis that required immune-suppressive doses of glucocorticoids to assist with management. A history of radiation pneumonitis in the radiation field is permitted as long as pneumonitis resolved $\geq 6$ months prior to randomisation

- Patients with active, known, or suspected autoimmune disease that has required systemic therapy in the past 2 years

- Patients with a condition requiring corticosteroid therapy (>10 mg prednisone/day or equivalent) within 14 days of randomisation

- Another malignancy that is progressing or requires treatment

- Uncontrolled infection with hepatitis B or hepatitis C or human immunodeficiency virus (HIV) or diagnosis of immunodeficiency

- Active infection requiring systemic therapy within 14 days prior to randomisation

- Prior therapy with anti-PD 1 or anti-PD L1

- Treatment-related immune-mediated AEs from immune-modulatory agents

- Receipt of an investigational drug or device within 30 days

- Receipt of a live vaccine within 30 days of planned start of study medication

- Major surgery or significant traumatic injury within 4 weeks prior to first dose

- Documented allergic or acute hypersensitivity reaction attributed to antibody treatments

- Known psychiatric or substance abuse disorder that would interfere with participation with the requirements of the study, including current use of any illicit drugs

- Pregnant or breast feeding women

- Women of childbearing potential or men who are unwilling to practice highly effective contraception prior to the initial dose/start of the first treatment, during the study, and for at least 6 months after the last dose

Note: Other protocol defined Inclusion/Exclusion criteria apply.

\section{Intervention}

Cemiplimab (Anti-PD-1 Antibody)

\section{Comparator}

Standard of care platinum-based chemotherapy

\section{Outcomes}

\section{Primary outcome measures}

- Overall survival (OS) (time frame: from date of randomisation until the date of death, assessed up to 68 months) 
- Progression-free survival (PFS) as assessed by a blinded Independent review committee (IRC) using RECIST 1.1 (time frame: from date of randomisation until the date of first documented progression or date of death from any cause, whichever came first, assessed up to 68 months). PFS as assessed by a blinded IRC using RECIST 1.1.

\section{Secondary outcome measures}

- Objective response rates (ORR) (time frame: from date of randomisation to the date of the first objectively-documented progression or the date of subsequent anti-cancer therapy, whichever comes first, up to 68 months). The number of patients with a best overall response (BOR) of confirmed complete response $(C R)$ or partial response $(P R)$ divided by the number of patients in the efficacy analysis set

- Best overall response (BOR) (time frame: from date of randomisation until the date of first documented progression or the date of subsequent anti-cancer therapy, whichever came first, assessed up to 68 months). The BOR, as determined by the IRC per RECIST 1.1

- Compare the duration of response (DOR) of cemiplimab versus platinum-based chemotherapies (time frame: from date of randomisation until the date of first documented progression or date of death from any cause, whichever came first, assessed up to 68 months). Duration of response will be defined as the time between the date of first response (CR or PR) to the date of the first documented tumour progression (per RECIST 1.1) or the date of subsequent anti-cancer therapy or death due to any cause, whichever comes first

- Change from baseline in quality of life (QoL) scores as assessed by the European Organization for the Research and Treatment of Cancer Quality of Life Questionnaire Core 30 (EORTC QLQ-C30) time frame: baseline up to 26 months after treatment)

- Change from baseline in lung cancer symptom scores as measured by the EORTC Lung Cancer 13 (EORTC QLQ-LC13) (time frame: baseline up to 26 months after treatment)

- Incidence of adverseeEvents (AEs) (time frame: baseline up to 68 months after treatment)

- Incidence of serious adverse events (SAEs) (time frame: baseline up to 68 months after treatment)

- Incidence of deaths (time frame: baseline up to 68 months after treatment)

- Incidence of laboratory abnormalities (time frame: baseline up to 68 months after treatment). Number of patients with laboratory abnormalities

- Measure concentrations of cemiplimab in serum (time frame: baseline up to 68 months after treatment). Maximum plasma concentration ( $\mathrm{Cmax}$ )

- Characterise the pharmacokinetics (PK) of cemiplimab (time frame: baseline up to 68 months after treatment). Area under the curve (AUC)

Notes

\section{Sponsorship source: Regeneron Pharmaceuticals}

ClinicalTrials.gov Identifier: NCT03088540

Study protocol: no protocol published

Other notes: $74 \%$ of people cross-over from chemotherapy to receive cemiplimab

\section{Risk of bias}

\begin{tabular}{lll}
\hline Bias & Authors' judgement & Support for judgement \\
\hline $\begin{array}{l}\text { Random sequence genera- } \\
\text { tion (selection bias) }\end{array}$ & Unclear risk & Randomisation was not reported \\
\hline $\begin{array}{l}\text { Allocation concealment } \\
\text { (selection bias) }\end{array}$ & Unclear risk & Method of assignment to intervention was not reported \\
\hline $\begin{array}{l}\text { Blinding of participants } \\
\text { and personnel (perfor- } \\
\text { mance bias) }\end{array}$ & High risk & $\begin{array}{l}\text { It is an open-label study. The absence of blinding could influence results of } \\
\text { sll outcomes primary outcomes (progression-free survival). }\end{array}$ \\
\hline
\end{tabular}


Sezer 2020 (Continued)

Blinding of outcome as- Unclear risk No information sessment (detection bias)

All outcomes

\begin{tabular}{lll}
$\begin{array}{l}\text { Incomplete outcome data } \\
\text { (attrition bias) } \\
\text { All outcomes }\end{array}$ & Unclear risk & $\begin{array}{l}\text { No full publication was available, trial primary outcomes were presented in an } \\
\text { abstract }\end{array}$ \\
\hline $\begin{array}{l}\text { Selective reporting (re- } \\
\text { porting bias) }\end{array}$ & Unclear risk & No information \\
\hline Other bias & High risk & $\begin{array}{l}\text { PD-L1 level } \geq 50 \% \text { was an inclusion criteria, however some participants were } \\
\text { retested and had PD-L1 expression }<50 \% \text {. Only OS and PFS data were report- } \\
\text { ed for people with } \geq 50 \% \text { PD-L1 level intention to treat population, the rest of } \\
\text { participants characteristics were presented for all participants regardless of } \\
\text { the PD-L1 level. }\end{array}$
\end{tabular}

AEs; adverse events; ALK: anaplastic lymphoma kinase ; CNS: central nervous system; CT: computed tompography; ; IVRS: interactive voice response system; MRI: magnetic resonance imaging; NSCLC: non-small cell lung cancer; ORR; objective response rate; OS; overall survival; PFS: progression-free survival; TMB: tumour mutational burden.

Characteristics of excluded studies [ordered by study ID]

\begin{tabular}{ll}
\hline Study & Reason for exclusion \\
\hline Huan 2019 & wrong study design \\
\hline Hui 2017a & wrong study design \\
\hline Leighl 2019 & wrong study design \\
\hline Mok 2017 & Wrong intervention \\
\hline Ready 2019 & wrong intervention \\
\hline
\end{tabular}

Characteristics of ongoing studies [ordered by study ID]

\section{EMPOWER-Lung 2}

$\begin{array}{ll}\text { Study name } & \text { A randomized, phase 3, open-label study of combinations of REGN2810 (Anti-PD-1 antibody), plat- } \\ \text { inum-based doublet chemotherapy, and ipilimumab (Anti-CTLA-4 Antibody) versus pembrolizum- } & \text { ab monotherapy in first-line treatment of patients with advanced or metastatic Non-Small Cell } \\ \text { Lung Cancer with tumors expressing PD-L1 } 1 \geq 50 \%\end{array}$

Methods

Open-label, parallel assignment, Phase 3 RCT

Participants 5 participants

\section{Inclusion criteria}

- Patients with histologically- or cytologically-documented squamous or non-squamous NSCLC with stage IIIB or stage IV disease, who received no prior systemic treatment for recurrent or metastatic NSCLC

- Availability of an archival ( $\leq 5$ months) or on-study obtained formalin-fixed, paraffin-embedded tumour tissue sample which has not previously been irradiated 
- Expression of PD-L1 in $\geq 50 \%$ of tumour cells determined by the commercially available assay performed by the central laboratory

- At least 1 radiographically-measurable lesion by computed tomography (CT) or magnetic resonance imaging (MRI) per RECIST 1.1 criteria. Target lesions may be located in a previously irradiated field if there is documented (radiographic) disease progression in that site

- Eastern Cooperative Oncology Group (ECOG) performance status of $\leq 1$

- Anticipated life expectancy of at least 3 months

\section{Exclusion criteria}

- Patients who have never smoked, defined as smoking $\leq 100$ cigarettes in a lifetime

- Active or untreated brain metastases or spinal cord compression

- Patients with tumour tested positive for epidermal growth factor receptor (EGFR) gene mutations, anaplastic lymphoma kinase (ALK) gene translocations, or C-ros oncogene receptor tyrosine kinase(ROS1) fusions

- Encephalitis, meningitis, or uncontrolled seizures in the year prior to informed consent

- History of interstitial lung disease (e.g. idiopathic pulmonary fibrosis or organising pneumonia), of active, noninfectious pneumonitis that required immune-suppressive doses of glucocorticoids to assist with management, or of pneumonitis within the last 5 years

- Ongoing or recent evidence of significant autoimmune disease that required treatment with systemic immunosuppressive treatments, which may suggest risk of immune-related treatment-emergent adverse events (irTEAEs)

- Patients with a condition requiring corticosteroid therapy (>10 mg prednisone/day or equivalent) within 14 days of randomisation

\section{Intervention}

REGN2810 plus ipilimumab

Other name: cemiplimab

\section{Intervention}

REGN2810 plus chemotherapy plus Ipilimumab

Other name: cemiplimab

\section{Comparator}

Pembrolizumab

Reference drug administered IV infusion

\section{Primary outcome measures}

- PFS as assessed by a blinded Independent Review Committee (IRC) based on Response Evaluation Criteria in Solid Tumors version 1.1 (RECIST 1.1) assessments (time frame: up to 32 months)

\section{Secondary outcome measures}

- Overall survival (OS) (time frame: up to 32 months)

- Objective response rate (ORR) (time frame: up to 32 months)

- Incidence of treatment-emergent adverse events (TEAEs) (time frame: up to 32 months)

- Incidence of dose-limiting toxicities (DLTs) (time frame: up to 32 months)

- Incidence of serious adverse events (SAEs) (time frame: up to 32 months)

- Incidence of deaths (time frame: up to 32 months)

- Incidence of laboratory abnormalities (time frame: up to 32 months)

- Overall survival (time frame: 12 months)

- Overall survival (time frame: 18 months) 
- Quality of life (Core 30 Questionnaire) (time frame: up to 32 months). As measured by the European Organization for Research and Treatment of Cancer Quality of Life Questionnaire Core 30 (EORTC QLQ-C3) four-point scale, with 1 as "not at all" and 4 as "very much"

- Quality of life (Lung Cancer 13 Questionnaire (timefFrame: up to 32 months) as measured by the Quality of Life Questionnaire Lung Cancer 13 (EORTC QLQ-LC13) to assess lung cancer-associated symptoms and treatment-related side effects among lung cancer patients.

Starting date

Actual study start date: June 4, 2018

Estimated study completion date: November 18, 2020

Contact information

Notes

ClinicalTrials.gov Identifier: NCT03515629

\section{ENERGY}

$\begin{array}{ll}\text { Study name } & \text { Randomized phase III study testing nivolumab and ipilimumab versus a carboplatin based doublet } \\ \text { in first line treatment of PS } 2 \text { or elderly (more than } 70 \text { years old). patients with advanced Non-small } \\ \text { Cell Lung Cancer }\end{array}$

\begin{tabular}{ll}
\hline Methods & Open-label, parallel assignment, Phase 3 RCT \\
\hline
\end{tabular}

Participants

\section{2 participants}

\section{Inclusion criteria}

- Signed written informed consent

- Cytologically- or histologically-proven NSCLC (adenocarcinoma, squamous cell carcinoma, largecell carcinoma)

- Stage IV or non-treatable by radiotherapy or surgery stage III (7th classification)

- No previous systemic chemotherapy for lung cancer, except in case of relapse after adjuvant treatment for localized disease with 6 months or more between end of previous chemotherapy and relapse

- Patients less than 70 years old and PS 2 or 70 years older PS 0 to 2

- Judged fit enough to receive a carboplatin based doublet according to ESMO guidelines

- Presence of at least one measurable target lesion (RECIST 1.1 rules) in a non-irradiated region and analysable by $\mathrm{CT}$

- Life expectancy superior at 12 weeks

- Prior radiation therapy is authorised if it involved less than $25 \%$ of the total bone marrow volume and finished 14 days before D1 of planned treatment

- Screening laboratory values must meet the following criteria and should be obtained within 14 days prior to randomisation/registration WBC superior or equal at 2000/ $\mu \mathrm{L}$ Neutrophils superior or equal at $1500 / \mu \mathrm{L}$ Platelets superior or equal at $100 \times 103 / \mu \mathrm{L}$ Hemoglobin superior at $10.0 \mathrm{~g} / \mathrm{dL}$. Serum creatinine inferior or equal at $1.5 \times$ ULN or creatinine clearance $(\mathrm{CrCl})$ superior or equal at $45 \mathrm{~mL} / \mathrm{min}$ (if using the Cockcroft-Gault formula ) AST/ALT inferior or equal at $3 \times$ ULN Total Bilirubin inferior or equal at $1.5 \times$ ULN (except Patients with Gilbert Syndrome, who can have total bilirubin inferior at $3.0 \mathrm{mg} / \mathrm{dL}$ )

- Availability of adequate FFPE tumour-derived material (tumour blocks or slides) from a biopsy, surgery or fine needle aspirate for analysis of PD-L1 testing by IHC

\section{Age and reproductive status}

- Women of childbearing potential (WOCBP) must use appropriate method(s) of contraception during treatment.

WOCBP should use an adequate method to avoid pregnancy :

Single or combined immune checkpoint inhibitors compared to first-line platinum-based chemotherapy with or without bevacizumab for 56 people with advanced non-small cell lung cancer (Review)

Copyright $\odot 2020$ The Cochrane Collaboration. Published by John Wiley \& Sons, Ltd. 
eNERGY (Continued)

- For 23 weeks (30 days plus the time required for nivolumab to undergo five half-lives) after the last dose of nivolumab + ipilimumab,

- For 4 weeks after the last dose of carboplatin + pemetrexed,

- For 5 weeks after the last dose of carboplatin e + paclitaxel.W

- Women of childbearing potential must have a negative serum or urine pregnancy test (minimum sensitivity $25 \mathrm{IU} / \mathrm{L}$ or equivalent units of HCG) within 24 hours prior to the start of treatment

- Women must not be breastfeeding. Men who are sexually active with WOCBP must use any contraceptive method with a failure rate of less than $1 \%$ per year during treatment Men will be instructed to adhere to contraception for a period of 31 weeks after the last dose of nivolumab + ipilimumab and with carboplatin e +pemetrexed or carboplatin e + paclitaxel up to 6 months thereafter.

\section{Exclusion criteria}

- Patients with other severe concurrent disorders that occurred during the prior six months before enrolment (myocardial infection, severe or unstable angor, coronarian or peripheric arterial bypass operation, NYHA class 3 or 4 congestive heart failure, transient or constituted cerebral ischaemic attack, at least grade 2 peripheral neuropathy, psychiatric or neurological disorders preventing the patient from understanding the trial, uncontrolled infections) are not eligible.

- Serious or uncontrolled systemic disease judged as incompatible with the protocol by the investigator

- Another previous or concomitant cancer, except for basocellulare cancer of the skin or treated cervical cancer in situ, or appropriately treated localized low-grade prostate cancer (Gleason score inferior at 6), unless the initial tumour was diagnosed and definitively treated more than 5 years previously, with no evidence of relapse.

- Known activating mutation of EGFR (del LREA exon 19, mutation L858R or L861X of exon 21, mutation G719A/S in exon 18) or EML4-ALK or ROS-1 translocation

- Superior at caval syndrome

- Uncontrolled infectious status

- All concurrent radiotherapy

- Concurrent administration of one or several other anti-tumour therapies.

- Psychological, familial, social or geographic difficulties preventing follow-up as defined by the protocol.

- Protected person (adults legally protected (under judicial protection, guardianship or supervision), person deprived of their liberty, pregnant woman, lactating woman and minor),

- Concurrent participation in another clinical trial

- Patients are excluded if they have active brain metastases or leptomeningeal metastases. Patients with brain metastases are eligible if metastases have been treated and there is no magnetic resonance imaging (MRI) evidence of progression for (lowest minimum is 4 weeks or more) after treatment is complete and within 28 days prior to the first dose of nivolumab and ipilimumab administration. There must also be no requirement for immunosuppressive doses of systemic corticosteroids (superior at $10 \mathrm{mg} /$ day prednisone equivalents) for at least 2 weeks prior to study drug administration.

- Patients should be excluded if they have an active, known or suspected autoimmune disease. Patients are permitted to enrol if they have vitiligo, type I diabetes mellitus, residual hypothyroidism due to autoimmune condition only requiring hormone replacement, psoriasis not requiring systemic treatment, or conditions not expected to recur in the absence of an external trigger

- Patients should be excluded if they have a condition requiring systemic treatment with either corticosteroids (superior at $10 \mathrm{mg}$ daily prednisone equivalents) or other immunosuppressive medications within 14 days of study drug administration. Inhaled or topical steroids and adrenal replacement doses superior at $10 \mathrm{mg}$ daily prednisone equivalents are permitted in the absence of active autoimmune disease.

- Patients should be excluded if they are positive test for hepatitis B virus surface antigen (HBV sAg) or hepatitis $\mathrm{C}$ virus ribonucleic acid (HCV antibody) indicating acute or chronic infection

- Patients should be excluded if they have known history of testing positive for human immunodeficiency virus (HIV) or known acquired immunodeficiency syndrome (AIDS)

- Patients should be excluded if they have a lung disease that is symptomatic or may interfere with the detection or management of suspected drug-related pulmonary toxicity

Single or combined immune checkpoint inhibitors compared to first-line platinum-based chemotherapy with or without bevacizumab for 
eNERGY (Continued)

- Allergies and Adverse Drug Reaction

- History of allergy to study drug components

- Severe spinal hypoplasia and/or hemorrhagic tumours.

Interventions

\section{Intervention}

Nivolumab + Ipilimumab. Nivolumab dosed intravenously over 30 minutes at $240 \mathrm{mg}$ every 2 weeks combined with Ipilimumab dosed intravenously over 30 minutes at $1 \mathrm{mg} / \mathrm{kg}$ every 6 weeks until disease progression, unacceptable toxicity, or other reasons specified in the protocol.

\section{Comparator}

Chemotherapy. Doublet of chemotherapy according to standard of care carboplatin (AUC 5) with a dose that will be capped to $700 \mathrm{mg}$ and pemetrexed $\left(500 \mathrm{mg} / \mathrm{m}^{2}\right)$ over 4 to 6 hours every three weeks (restricted to non-squamous histology) or carboplatin (AUC 6) with a dose that will be capped to $700 \mathrm{mg}$ and paclitaxel $\left(90 \mathrm{mg} / \mathrm{m}^{2}\right)$ D1 D8 D15 over 4 to 6 hours every 4 weeks, with a maximum of 4 cycles of carboplatin based doublet, and the possibility to use maintenance with pemetrexed.

\section{Outcomes Primary outcome measures}

- Overall survival: (time frame: from date of randomisation until the date of date of death from any cause, whichever came first, assessed up to 3 years maximum)

\section{Secondary outcome measures}

- Survival rate (time frame: 1 year)

- Objective response rate (time frame: 2 years) according to RECIST 1.1

- Progression-free survival (time frame: from date of randomisation until the date of first documented progression or date of death from any cause, whichever came first, assessed up to 3 years maximum)

- Safety (time frame: 2 years) according to CTCAE version 4.0

- Tolerability (time frame: 2 years) according to CTCAE version 4.0

- Quality of life (time frame: from date of randomisation until the date of first documented progression or date of death from any cause, whichever came first, assessed up to 3 years maximum] according to EQ-5D questionnaire)

- Quality of life (time frame: from date of randomisation until the date of first documented progression or date of death from any cause, whichever came first, assessed up to 3 years maximum] according to EORTC QLQ-ELD14 questionnaire)

- PD-L1 (time frame: 2 years) testing by immunochemistry

- Geriatric evaluation (time frame: inclusion and 2 months) according to geriatric mini data set

Starting date

Estimated study start date: December 2017

Estimated study completion date: June 2022

Contact information

Notes ClinicalTrials.gov Identifier: NCT03351361

\section{IPSOS}

Study name

A phase III, open-label, multicenter, randomized study to investigate the efficacy and safety of atezolizumab compared with chemotherapy in patients with treatment naïve advanced or recurrent (stage IIIb not amenable for multimodality treatment) or metastatic (stage IV) Non-Small Cell Lung Cancer who are deemed unsuitable for platinum-containing therapy 
IPSOS (Continued)

Methods

Open-label, parallel assignment, Phase 3 RCT

Participants

441 participants

\section{Inclusion criteria}

- Histologically- or cytologically-confirmed diagnosis of advanced or recurrent (Stage IIIB not amenable for multimodality treatment) or metastatic (Stage IV) NSCLC as per the American Joint Committee on Cancer (AJCC) 7th edition

- No sensitising epidermal growth factor receptor (EGFR) mutation (L858R or exon 19 deletions) or anaplastic lymphoma kinase (ALK) fusion oncogene detected

- No prior systemic treatment for advanced or recurrent (Stage IIIB not amenable for multimodality treatment) or metastatic (Stage IV) NSCLC as per the AJCC 7th edition

- Life expectancy greater than or equal to $(>/=) 8$ weeks

- Deemed unsuitable by the investigator for any platinum-doublet chemotherapy due to poor performance status (ECOG performance status of 2-3). However, participants $>=70$ years of age who have an ECOG PS of 0 or 1 may be included due to: a) substantial comorbidities; b) contraindication(s) for any platinum-doublet chemotherapy

- Representative formalin-fixed paraffin-embedded (FPPE) tumour tissue block obtained during course of disease (archival tissue) or at screening

- Participants with treated, asymptomatic central nervous system (CNS) metastases are eligible, provided they meet all of the following criteria: Measurable disease outside CNS; Only supratentorial and cerebellar metastases allowed; No ongoing requirement for corticosteroids as therapy for CNS disease; No stereotactic radiation within 7 days or whole-brain radiation within 14 days prior to randomisation; No evidence of interim progression between the completion of CNS-directed therapy and the screening radiographic study

- Adequate hematologic and end organ function

- Female participants of childbearing potential randomised to the atezolizumab treatment arm agree to use protocol defined methods of contraception

\section{Exclusion criteria}

\section{Cancer-specific exclusion criteria}

- Participants younger than 70 years who have an ECOG performance status of 0 or 1

- Active or untreated CNS metastases as determined by computed tomography (CT) or magnetic resonance imaging (MRI) evaluation of the brain during screening and prior radiographic assessments

- Uncontrolled tumour-related pain

- Uncontrolled pleural effusion, pericardial effusion, or ascites requiring recurrent drainage procedures (once monthly or more frequently)

- Uncontrolled or symptomatic hypercalcaemia (ionised calcium $>1.5 \mathrm{mmol} / \mathrm{L}$ or calcium $>12 \mathrm{mg}$ / $\mathrm{dL}$ or corrected serum calcium $>\mathrm{ULN}$ )

- History of other malignancy within 5 years prior to screening, with the exception of those with a negligible risk of metastasis or death treated with expected curative outcome

- National Cancer Institute Common Terminology Criteria for Adverse Events (NCI CTCAE) version 4.0 (v4.0) Grade 3 or higher toxicities due to any prior therapy (example (e.g. radiotherapy) (excluding alopecia), which have not shown improvement and are strictly considered to interfere with current study medication

- Participants who have received prior neo-adjuvant, adjuvant chemotherapy, radiotherapy, or chemoradiotherapy with curative

\section{Interventions}

\section{Atezolizumab}

Participants will receive atezolizumab 1200 milligrams (mg) intravenous (IV) infusion on Day 1 of each 21-day cycle until loss of clinical benefit, unacceptable toxicity, participant or physician decision to discontinue, or death.

Single or combined immune checkpoint inhibitors compared to first-line platinum-based chemotherapy with or without bevacizumab for 
IPSOS (Continued)

\section{Comparators}

Single-agent chemotherapy (vinorelbine or gemcitabine)

Participants will receive single-agent chemotherapy; either vinorelbine oral or IV, or gemcitabine IV, according to the label based on investigator's choice.

Outcomes

\section{Primary outcomes}

- Overall survival (time frame: from randomisation up to death from any cause (up to approximately 3.5 years)

\section{Secondary outcomes}

- Percentage of participants who are alive at specified time points (time frame: $6,12,18$ and 24 months)

- Percentage of participants with objective response (OR), as determined by the Investigator using Response Evaluation Criteria In Solid Tumors (RECIST) Version 1.1 (v1.1) (time frame: from randomisation to the first occurrence of disease progression or death from any cause, whichever occurs first (up to approximately 3.5 years)). Objective response is defined as partial response (PR) plus complete response (CR).

- Progression-free survival (PFS), as determined by the Investigator using RECIST v1.1 (time frame: from randomisation to the first occurrence of disease progression or death from any cause, whichever occurs first (up to approximately 3.5 years))

- Duration of response, as determined by the Investigator using RECIST v1.1 (time frame: time from the first occurrence of a documented objective response to the time of disease progression or death from any cause, whichever occurs first (up to approximately 3.5 years))

- Percentage of participants with adverse events (AEs) (time frame: from randomisation up to approximately 3.5 years)

- Change from baseline in European Organisation for Research and Treatment of Cancer Quality of Life Questionnaire - Core 30 (EORTC-QLQ-C30) Score (time frame: baseline, day 1 of each treatment cycle up to 30 days after last dose (up to approximately 3.5 years) (cycle length $=21$ days))

- Change from baseline in EORTC QLQ Supplementary Lung Cancer Module 13 (EORTC QLQ-LC13) Score (time frame: baseline, day 1 of each treatment cycle up to 30 days after last dose (up to approximately 3.5 years) (Cycle length $=21$ days)). Time to deterioration in patient-reported lung cancer symptoms as assessed by EORTC QLQ-C30 Score (time frame: from baseline up to approximately 3.5 years)

- Time to deterioration in patient-reported lung cancer symptoms as assessed by EORTC QLQ-LC13 Score (time frame: from baseline up to approximately 3.5 years)
Actual study start date: September 11, 2017

Estimated study completion date: January 20, 2021

Contact information

Notes ClinicalTrials.gov Identifier: NCT03191786

\section{JAVELIN Lung 100}

Study name

A phase III, open-label, multicenter trial of avelumab (MSB0010718C) versus platinum-based doublet as a first-line treatment of recurrent or stage IV PD-L1+ Non-small Cell Lung Cancer

\begin{tabular}{ll}
\hline Methods & Open-label, parallel assignment, Phase 3 RCT \\
\hline Participants & 1224 participants \\
& Inclusion criteria \\
\hline
\end{tabular}

Single or combined immune checkpoint inhibitors compared to first-line platinum-based chemotherapy with or without bevacizumab for 60 people with advanced non-small cell lung cancer (Review)

Copyright (c) 2020 The Cochrane Collaboration. Published by John Wiley \& Sons, Ltd. 
- Male or female participants aged greater than or equal to (>=) 18 years

- With Eastern Cooperative Oncology Group Performance Status (ECOG PS) of 0 to 1 at trial entry

- At least 1 measurable tumour lesion

- With histologically-confirmed metastatic or recurrent (Stage IV) non-small cell lung cancer (NS(LC)

- With availability of a recently-obtained, formalin-fixed, paraffin-embedded (FFPE) tissue sample containing tumour (biopsy from a non-irradiated area preferably within 6 months) or a minimum number of 10 (preferably 25) unstained tumour slides cut within 1 week, and suitable for PD-L1 expression assessment

- Participants must not have received any treatment for systemic lung cancer, and have an estimated life expectancy of more than 12 weeks

- Other protocol defined criteria could apply

\section{ExclusioncCriteria}

- Participants whose disease harbours a EGFR mutation, or anaplastic lymphoma kinase (ALK) rearrangement are not eligible.

- Other exclusion criteria include prior therapy with any antibody or drug targeting T cell coregulatory proteins, concurrent anticancer treatment, or immunosuppressive agents

- Known severe hypersensitivity reactions to monoclonal antibodies (Grade $>=3 \mathrm{NCI}$ CTCAE $\vee 4.03$ ), history of anaphylaxis, or uncontrolled asthma (that is, 3 or more features of partially controlled asthma), and persisting toxicity related to prior therapy of Grade > 1 NCl-CTCAE v 4.03.

- Participants with brain metastases are excluded, except those meeting the following criteria: brain metastases that have been treated locally and are clinically stable for at least 2 weeks prior to randomisation, participants must be either off steroids or on a stable or decreasing dose of $<=$ $10 \mathrm{mg}$ daily prednisone (or equivalent), and do not have ongoing neurological symptoms that are related to the brain localisation of the disease.

- Other protocol defined criteria could apply

Interventions

\section{Intervention}

\section{Avelumab}

Participants will be administered with avelumab at a dose of 10 milligram per kilogram $(\mathrm{mg} / \mathrm{kg}) 1$ hour intravenous (IV) infusion once every 2 weeks until disease progression or unacceptable toxicities.

\section{Other Names:Anti-PD-L1; MSB0010718C}

\section{Comparator}

Platinum-containing chemotherapy regimen

Platinum-containing chemotherapy regimen: Investigator's choice platinum containing chemotherapy regimen to be administered consisting of one of the following: non-squamous tumour histology Pemetrexed (500 milligram per metre square $\left.\left[\mathrm{mg} / \mathrm{m}^{\wedge} 2\right]\right)+$ cisplatin $\left(75 \mathrm{mg} / \mathrm{m}^{\wedge} 2\right)$ or Pemetrexed $\left(500 \mathrm{mg} / \mathrm{m}^{\wedge} 2\right)+$ carboplatin (AUC $6 \mathrm{mg} / \mathrm{mL}^{\star} \mathrm{min}$ ) Squamous tumour histology Paclitaxel $\left(200 \mathrm{mg} / \mathrm{m}^{\wedge} 2\right)+$ carboplatin (AUC $\left.6 \mathrm{mg} / \mathrm{mL}^{\star} \mathrm{min}\right)$ Gemcitabine $\left(1250 \mathrm{mg} / \mathrm{m}^{\wedge} 2\right)+$ cisplatin $(75 \mathrm{mg} /$ $\mathrm{m}^{\wedge} 2$ ) Gemcitabine (1000 mg/m²)+carboplatin (AUC $5 \mathrm{mg} / \mathrm{mL}^{\star} \mathrm{min}$ )

Outcomes

\section{Primary outcome measures}

- Progressionf-free Survival (PFS) in participants with high PD-L1 + Tumor Expression based on an Independent Review Committee (IRC) Assessment According to RECIST 1.1 (time frame: time from date of randomisation until PD or death, assessed up to 56 months)

- Overall survival (OS) in participants with high PD-L1 + Tumor Expression (time frame: time from date of randomisation until death, assessed up to 56 months)

\section{Secondary outcome measures}


- Best Overall Response (BOR) as adjudicated by the IRC (time frame: time from date of randomisation up to 56 months ]BOR will be determined according to RECIST 1.1.

- Duration of Response (DOR) according to RECIST 1.1 (time frame: time from date of randomisation up to 56 months ]DOR will be determined according to RECIST 1.1

- European Quality Of Life 5-dimensions (EQ-5D-5L) Health Outcome Questionnaire (time frame: up to 56 months). The EQ-5D-5L Health Outcome Questionnaire is a measure of health status that provides a simple descriptive profile and a single index value.

- European Organization for the Research and Treatment of Cancer Quality of Life (EORTC QLQ-C30) Global Health Status (time frame: up to 56 months) EORTC QLQ-C30 is a 30-question tool used to assess the overall quality of life $(\mathrm{Q} O \mathrm{~L})$ in cancer participants.

- European Organization for the Research and Treatment of Cancer Quality of Life Questionnaire Lung Cancer 13 (EORTC QLQ-LC13) (time frame: up to 56 months)

- EORTC QLQ-LC13 consists of 13 questions relating to disease symptoms specific to lung cancer and treatment side effects typical of treatment with chemotherapy and radiotherapy.

- Number of participants with Treatment-Emergent Adverse Events (TEAEs) according to the National Cancer Institute Common Terminology Criteria for Adverse Events (NCI-CTCAE) Version 4.03 (time frame: up to 56 months)

- Number of participants with abnormalities in safety laboratory tests as graded by NCI-CTCAE (Version 4.03) (time frame: up to 56 months)

- Number of participants with abnormalities in vital signs, physical examination, and Eastern Cooperative Oncology Group (ECOG) PS. (time frame: up to 56 months)

- Number of participants with abnormalities in 12-lead ECG time frame: up to 56 months)

- EORTC QLQ-LC13 consists of 13 questions relating to disease symptoms specific to lung cancer and treatment side effects typical of treatment with chemotherapy and radiotherapy.

- Number of participants with Treatment-Emergent Adverse Events (TEAEs) according to the National Cancer Institute Common Terminology Criteria for Adverse Events (NCI-CTCAE) Version 4.03 (time frame: up to 56 months)

- Number of participants with abnormalities in safety laboratory tests as graded by NCI-CTCAE (Version 4.03) (time frame: up to 56 months)

- Number of participants with abnormalities in vital signs, physical examination, and Eastern Cooperative Oncology Group (ECOG) PS. (time frame: up to 56 months)

- Number of participants with abnormalities in 12-lead ECG time frame: up to 56 months)

Starting date

Actual study start date: October 29, 2015

Estimated study completion date: June 3, 2025

Contact information

Notes $\quad$ https://clinicaltrials.gov/ct2/show/NCT02576574

\section{KEYNOTE-598}

Study name

A phase 3, randomized, double-blind study of pembrolizumab plus ipilimumab vs pembrolizumab plus placebo in previously untreated, stage IV, metastatic Non-small Cell Lung Cancer subjects whose tumors are PD-L1 positive (TPS $\geq 50 \%$ ) (KEYNOTE-598)

Methods Open-label, parallel assignment, Phase 3 RCT

Participants 548 participants

\section{Inclusion criteria}

- Has a histologically- or cytologically-confirmed diagnosis of Stage IV metastatic NSCLC (American Joint Committee on Cancer version 8) 
- Has measurable disease per RECIST 1.1 as determined by investigator

- Has Eastern Cooperative Oncology Group (ECOG) performance status of 0 or 1

- Has a life expectancy of $>3$ months

- Has provided archival tumour tissue sample or newly obtained core or excisional biopsy of a tumour lesion not previously irradiated

- Female participants of childbearing potential must have a negative serum pregnancy test within 72 hours prior to receiving the first dose of study therapy

- Female and male participants of reproductive potential must agree to use contraception starting from the first dose of study medication, throughout the study period, and for up to 120 days after the last dose of study medication

- Male participants must refrain from donating sperm starting from the first dose of study medication, throughout the study period, and for up to 120 days after the last dose of study medication

\section{Exclusion criteria}

- Has received prior systemic chemotherapy/other targeted or biological antineoplastic therapy treatment for their Stage IV metastatic NSCLC

- Has a tumour that harbours an epidermal growth factor receptor (EGFR)-sensitising (activating) mutation or an anaplastic lymphoma kinase (ALK) translocation

- Is currently participating in or has participated in a trial of an investigational agent or has used an investigational device within 4 weeks prior to the first dose of study therapy

- Has received prior therapy with an anti-Programmed Cell Death Receptor 1 (PD-1), Programmed Cell Death Receptor Ligand 1 (anti-PD-L1), or anti- Programmed Cell Death Receptor Ligand 2 (PDL2) agent or with an agent directed to another stimulatory or co-inhibitory T-cell receptor (e.g., cytotoxic T-lymphocyte-associated protein 4 (CTLA-4], OX-40, CD137)

- Has received prior radiotherapy within 2 weeks of start of study therapy or received lung radiation therapy of $>30$ Gray (Gy) within 6 months of the first dose of study therapy

- Has recovered from all radiation-related toxicities, does not require corticosteroids, and has not had radiation pneumonitis

- Is receiving systemic steroid therapy $\leq 7$ days prior to the first dose of study therapy or receiving any other form of immunosuppressive medication

- Has a known additional malignancy that is progressing or has required active treatment within the past 3 years with the exception of curatively treated basal cell carcinoma of the skin, squamous cell carcinoma of the skin and/or curatively resected in situ cancers

- Has known untreated central nervous system (CNS) metastases and/or carcinomatous meningitis

- Has an active autoimmune disease that has required systemic treatment in past 2 years (i.e., with use of disease-modifying agents, corticosteroids, or immunosuppressive drugs)

- Has a diagnosis of immunodeficiency or is receiving chronic systemic steroid therapy (i.e., doses exceeding $10 \mathrm{mg}$ daily of prednisone equivalent) or any other form of immunosuppressive therapy within 7 days prior the first dose of study therapy

- Has a history of (non-infectious) pneumonitis that required systemic steroids or current pneumonitis/interstitial lung disease

- Has had an allogeneic tissue/solid organ transplant

- Has received a live vaccine within 30 days prior to the first dose of study therapy

- Has an active infection requiring systemic therapy

- Has a known history of human immunodeficiency virus (HIV) infection

- Has a known history of hepatitis $B$ or known active hepatitis $C$ virus infection

- Has a known history of active tuberculosis

- Has known psychiatric or substance abuse disorders that would interfere with cooperating with the requirements of the trial

- Is a regular user of any illicit drugs or had a recent history of substance abuse

- Is pregnant or breast feeding or expecting to conceive or father starting from the first dose of study medication, throughout the study period, and for up to 120 days after the last dose of study medication

- Has severe hypersensitivity to pembrolizumab and/or any of its excipients and/or to ipilimumab and/or any of its excipients

Single or combined immune checkpoint inhibitors compared to first-line platinum-based chemotherapy with or without bevacizumab for 
KEYNOTE-598 (Continued)

- Has a ROS1 translocation

Interventions

\section{Intervention}

pembrolizumab + ipilimumab Participants receive $200 \mathrm{mg}$ of pembrolizumab by intravenous (IV) infusion on Day 1 of each 3-week cycle for up to 35 cycles of treatment plus $1 \mathrm{mg} / \mathrm{kg}$ of ipilimumab by IV infusion on Day 1 of each 6-week cycle for up to 18 cycles of treatment.

\section{Comparator:}

pembrolizumab + placebo Participants receive $200 \mathrm{mg}$ of pembrolizumab by IV infusion on Day 1 of each 3-week cycle for up to 35 cycles of treatment plus placebo by IV infusion on Day 1 of each 6week cycle for up to 18 cycles of treatment.

\section{Outcomes}

\section{Primary outcome measures}

- Overall survival (OS) (time frame: up to approximately 2 years). OS is the time from randomisation to death due to any cause.

- Progression-free survival (PFS) (time frame: up to approximately 2 years). PFS is the time from randomisation to first documented disease progression per Response Evaluation Criteria in Solid Tumors Version 1.1 (RECIST 1.1) by Blinded Independent Central Review (BICR).

\section{Secondary outcome measures}

- Objective response rate (ORR) (time frame: up to approximately 2 years). ORR is the proportion of the participants who achieve complete response (CR) or partial response (PR) per RECIST 1.1 by BICR.

- Duration of response (DOR) (time frame: up to approximately 2 years). DOR is the time from first documented evidence of CR or PR per RECIST 1.1 by BICR until disease progression per RECIST 1.1 by BICR or death.

- Time to true deterioration (TTD) in cough, pain in chest, and shortness of breath (time frame: on study day 1 prior to initiation of study therapy (baseline) and up to approximately 2 years). Time to true deterioration is defined as the time to the first onset of a 10-point or greater score decrease from study day 1 prior to initiation of study therapy (baseline) in any one of the 3 symptoms, confirmed by a second adjacent 10-point or greater score decrease from baseline.

- Incidence of adverse events (AEs) (time frame: from time of signing the informed consent form (ICF) until the end of follow-up (up to approximately 118 Weeks)). Percentage of participants experiencing any unfavourable and unintended sign, symptom, disease, or worsening of pre-existing condition temporally associated with study therapy and irrespective of causality to study therapy.

- Incidence of discontinuations (time frame: from time of signing the ICF until the end of study therapy (up to approximately 105 Weeks)). Percentage of participants discontinuing study drug due to an $A E$.

Starting date

Actual study start date: December 14, 2017

Estimated study completion date: February 2024

Contact information

Notes $\quad$ https://clinicaltrials.gov/ct2/show/NCT03302234

\section{MILES 5}

\section{Study name}

A study comparing immunotherapy with chemotherapy in the treatment of elderly patients with advanced NSCLC (MILES-5) 
MILES 5 (Continued)

Participants

\section{0 participants}

\section{Inclusion Criteria}

- Male or female $>=70$ years of age.

- Histological documentation of primary squamous or non squamous non-small cell lung carcinoma.

- Availability of archived tumour tissue block or newly cut unstained slides for PD-L1 determination.

- Stage IV or IIIB disease with supraclavear metastatic nodes (according to TNM 7th edition).

- Clinical or radiological evidence of disease (at least one measurable or non measurable lesion).

- ECOG performance status 0 to 1 .

- Life expectancy $>3$ months.

- Adequate renal and hepatic function, defined as:Total serum bilirubin $\leq 1.5$ institutional ULN.AST and/or ALT $\leq 2.5 \times$ ULN for the institution (or $\leq 5 \times$ ULN if liver metastases are present)Serum creatinine $\leq 1.5 \times$ ULN for the institution (or calculated creatinine clearance $\geq 40 \mathrm{~mL} / \mathrm{min} / 1.73 \mathrm{~m} 2$ ).

- Adequate bone marrow function, defined as:Haemoglobin $<=9.0 \mathrm{~g} / \mathrm{dLAbsolute}$ neutrophils count (ANC) >= $1.5 \times 109 / \mathrm{L}$ (> 1500 per mm3)Platelet count <= $100 \times 109 / \mathrm{L}(>100,000$ per mm3).

- Written informed consent obtained from the participant prior to performing any protocol-related procedures, including screening evaluations.

\section{Exclusion criteria}

\section{Cancer related}

- Activating epidermal growth factor receptor mutation (exon19 deletion or exon 21 L858R mutation or other activating/sensitising mutations).

- ALK or ROS1 positive (immunohistochemistry or FISH)

- Mixed small-cell lung cancer and NSCLC histology.Prior, current or planned treatment related

- Prior chemotherapy or any other medical treatment for advanced NSCLC (previous neoadjuvant or adjuvant chemotherapy is allowed if $>6$ months previously).

- Prior exposure to immunomodulatory therapy, including, but not limited to, other anti-cytotoxic T-lymphocyte-associated antigen 4 (CTLA-4), anti-programmed cell death1 (PD-1), anti-programmed cell death ligand 1 (PD-L1), or anti PD-L2 antibodies.

- Current or prior use of immunosuppressive medication within 14 days before the first dose of study treatment (intranasal and inhaled corticosteroids at physiological doses not exceeding 10 $\mathrm{mg}$ /day of prednisone or an equivalent corticosteroid are allowed).

- Any concurrent investigational product or other anticancer treatment.Prior or concomitant conditions or procedures related

- Active or prior documented autoimmune disease within the past 2 years participants with vitiligo, Grave's disease, or psoriasis not requiring systemic treatment within the past 2 years, are not excluded).

- Active or prior documented inflammatory bowel disease (e.g., Crohn's disease, ulcerative colitis)

- History of allogeneic organ transplant

- History of active primary immunodeficiency.

- Active infection, including tuberculosis (clinical evaluation that includes clinical history, physical examination and radiographic findings, and TB testing in line with local practice), hepatitis $B$ (known positive HBV surface antigen ( $\mathrm{HBsAg}$ ) result), hepatitis $\mathrm{C}$, or human immunodeficiency virus (positive HIV $1 / 2$ antibodies). Patients with a past or resolved HBV infection (defined as the presence of hepatitis B core antibody (anti-HBc) and absence of $\mathrm{HBsAg}$ ) are eligible. Patients positive for hepatitis $\mathrm{C}(\mathrm{HCV})$ antibody are eligible only if polymerase chain reaction is negative for HCV RNA.

- Receipt of live attenuated vaccine within 30 days prior to the first dose of study drugs.

- Patients with previous malignancies (except for adequately treated carcinoma in situ of the cervix or basal or squamous cell skin cancer or surgically resected prostate cancer with normal PSA) are excluded only if the histological diagnosis of the current disease does not definitely support the pulmonary origin. 
- Brain metastases or spinal cord compression, unless asymptomatic, previously treated, and stable off steroids and anti-convulsants for at least one month prior to study entry.

- Leptomeningeal carcinomatosis

- Clinically significant cardiovascular disease, including:Myocardial infarction or unstable angina pectoris within $<6$ months prior to the first study treatmentNew York Heart Association (NYHA) grade II or greater congestive heart failure (CHF) Uncontrolled hypertensionSerious cardiac arrhythmia requiring medication (with the exception of atrial fibrillation or paroxysmal supraventricular tachycardia)Peripheral vascular disease > grade 3 (i.e. symptomatic and interfering with activities of daily living requiring repair or revision)Mean QT interval corrected for heart rate (QTc) $\geq 470$ ms calculated from 3 electrocardiograms (ECGs) using Fredericia's Correction.

- Serious active infection requiring i.v. antibiotics at enrolment.

- Known hypersensitivity to any of the study drugs or excipients.

- Evidence of any other concomitant pathologies, physical examination or laboratory findings (including but not limited to active peptic ulcer disease or gastritis, active bleeding diatheses or psychiatric illness) or social situation that may interfere with the planned treatment, affect patient compliance or place the patient at high risk from treatment related complications.

- Radiotherapy treatment to the chest or to more than $30 \%$ of the bone marrow or with a wide field of radiation within 4 weeks of the first dose of study treatment (patients who have had radiotherapy $\geq 4$ weeks prior to the first dose of study treatment, but who are still experiencing acute toxic effects of radiotherapy are also excluded).

- Major surgical procedure within 28 days prior to the first dose of study drugs.

- Male patients of reproductive potential who are not willing to employ effective birth control from screening to 180 days after the last dose of study treatment.

\section{Interventions}

Durvalumab monotherapy (anti-PDL1 antibody) followed at progression by standard of care chemotherapy

Durvalumab (anti-PDl1 antibody ) + tremelimumab (anti CTLA-4 antibody) followed at progression by standard of care chemotherapy

\section{Comparator:}

Standard of care chemotherapy followed at progression by durvalumab 8anti-PDL1 antibody)

\section{Primary outcome measures}

12-month overall survival

December 20, 2018

Estimated study completion date: June 2023
Contact information

ClinicalTrials.gov Identifier: NCT03975114

\section{NEPTUNE}

\begin{tabular}{ll}
\hline Study name & $\begin{array}{l}\text { Study of 1st line therapy study of durvalumab with tremelimumab versus SoC in Non Small-Cell } \\
\text { Lung Cancer (NSCLC) (NEPTUNE). }\end{array}$ \\
\hline Methods & Open-label, multicentre, Phase 3 RCT \\
\hline Participants & $\begin{array}{l}\text { 953 participants } \\
\text { NCT03191786 }\end{array}$
\end{tabular}

Single or combined immune checkpoint inhibitors compared to first-line platinum-based chemotherapy with or without bevacizumab for 66 people with advanced non-small cell lung cancer (Review)

Copyright (c) 2020 The Cochrane Collaboration. Published by John Wiley \& Sons, Ltd. 
NEPTUNE (Continued)

\section{Inclusion criteria}

- Aged at least 18 years

- Documented evidence of Stage IV NSCLC

- No activating EGFR mutation or ALK rearrangement

- No prior chemotherapy or any other systemic therapy for recurrent/metastatic NSCLC

- World Health Organization (WHO) Performance Status of 0 or 1

- No Prior exposure to IMT, including, but not limited to, other antiCTLA4, antiPD1, anti PDL1,or antiPDL2 antibodies, excluding therapeutic anticancer vaccines

\section{Exclusion criteria}

Patients should not enter the study if any of the following exclusion criteria are fulfilled.

- Mixed small cell lung cancer and NSCLC histology, sarcomatoid variant

- Brain metastases or spinal cord compression unless the patient is stable (asymptomatic; no evidence of new or emerging brain metastases) and off steroids for at least 14 days prior to start of study treatment.

- Active or prior documented inflammatory bowel disease (e.g., Crohn's disease, ulcerative colitis)

Interventions

\section{Intervention}

Durvalumab (PD-L1 monoclonal antibody) + Tremelimumab (monoclonal antibody directed against CTLA-4)

\section{Comparator:}

Standard of Care chemotherapy treatment

Outcomes Primary outcomes

Overall survival (OS), time frame up to 4 years after first patient randomised

\section{Secondary outcomes:}

- OS in patients with PD-L1-negative NSCLC, time Frame: up to 4 years after first patient randomised

- Progression-free survival (PFS), time Frame: up to 4 years after first patient randomised

- Objective response rate (ORR), Time Frame: up to 4 years after first patient randomised

- Duration of response (DoR)

- Proportion of patients alive at 18 months (OS18)

- Proportion of patients alive and progression free at 12 months (APF12)

- Progression-free survival after subsequent anticancer therapy (PFS2).

- PK parameters

- Measuring the immunogenicity of durvalumab and tremelimumab by measuring the presence of Anti-drug Antibodies

- Proportion of patients alive at 12 months (OS12).

Other outcomes

Treatment-related adverse events as assessed by CTCAE v4.03

Starting date November 3, 2015

Estimated study completion date: August 22, 2019

Contact information 
PEARL

Study name

Methods

Participants
A phase III randomized, open-label, multi-center study of durvalumab (MEDI4736) versus standard of care (SoC) platinum-based chemotherapy as first line treatment in patients with PD-L1-High Expression advanced Non Small-Cell Lung Cancer
662 participants

\section{Inclusion criteria}

- Aged at least 18 years

- Documented evidence of Stage IV NSCLC

- No sensitising EGFR mutation and ALK rearrangement

- PD-L1 expression positive

- Eastern Cooperative Oncology Group (ECOG) performance status of 0 or 1

\section{Exclusion criteria}

- Prior chemotherapy or any other systemic therapy for advanced NSCLC

- Prior exposure to immune-mediated therapy, including, but not limited to, other anti-cytotoxic T-lymphocyte-associated antigen 4 (CTLA-4), anti-programmed cell death1 (PD-1), anti-programmed cell death ligand 1 (PD-L1), or anti PD-L2 antibodies, excluding therapeutic anticancer vaccines

- Brain metastases or spinal cord compression unless the patient is stable and off steroids for at least 14 days prior to start of study treatment

- Mixed small-cell lung cancer and NSCLC histology, sarcomatoid variant

- Active or prior documented autoimmune or inflammatory disorders (e.g., colitis or Crohn's disease]

Interventions

\section{Intervention}

Durvalumab (MEDI4736)

Anti-PD-L1 monoclonal Antibody monotherapy

\section{Comparator:}

Standard of care: platinum-based chemotherapy

\section{Primary outcome measures}

- The efficacy of Durvalumab therapy compared to SoC in terms of overall survival (OS) in patients with NSCLC (timefFrame: 4 years)

\section{Secondary outcome measures}

- The efficacy of Durvalumab compared to SoC in terms of Objective response rate (ORR) (time frame: 4 years)

- The efficacy of Durvalumab compared to SoC in terms of Duration of response (DoR) (time frame: 4 years)

- The efficacy of Durvalumab compared to SoC in terms of A Proportion of patients alive and progression free at 12 months from randomisation (APF12) (time frame: 12 months)

- The efficacy of Durvalumab compared to SoC in terms of progression-free survival after subsequent anticancer therapy (PFS2) (time frame: 4 years)

- Disease-related symptoms and health-related quality of life in participants treated with durvalumab compared to SoC using the European Organization for Research and Treatment of Cancer Quality of Life Questionnaire (time frame: 4 years) 
PEARL (Continued)

- The immunogenicity of durvalumab by measuring the presence of Anti-drug Antibodies (time frame: 4 years)

- The efficacy of Durvalumab therapy compared to SoC in terms of progress-free survival (PFS) in patients with NSCLC (time frame: 4 years)

- The efficacy of Durvalumab therapy compared to SoC in terms of Overall Survival (OS) in PD-L1 high patients with NSCLC (time frame: 4 years)

\section{Other outcome measures}

- The Incidence of Treatment-Emergent Adverse Events assessed by Common Terminology Criteria for Adverse Event (CTCAE) v4.03 for participants receiving Durvalumab therapy or SoC (time frame: 4 years)

Starting date Actual study start date: January 2, 2017

Estimated study completion date: December 31, 2020

Contact information

Notes ClinicalTrials.gov Identifier: NCT03003962

DATA AND ANALYSES

\section{Comparison 1. Single immunecheckpoint inhibitors vs chemotherapy}

\begin{tabular}{|c|c|c|c|c|}
\hline Outcome or subgroup title & $\begin{array}{l}\text { No. of } \\
\text { studies }\end{array}$ & $\begin{array}{l}\text { No. of } \\
\text { partici- } \\
\text { pants }\end{array}$ & Statistical method & Effect size \\
\hline $\begin{array}{l}1.1 \text { Overall survival by PD-L1 expres- } \\
\text { sion }\end{array}$ & 6 & & Hazard Ratio (IV, Random, 95\% Cl) & Subtotals only \\
\hline 1.1.1 PD-L1 TPS $<1 \%$ & 1 & 178 & Hazard Ratio (IV, Random, 95\% CI) & $1.18[0.86,1.61]$ \\
\hline 1.1.2 PD-L1 TPS $\geq 1 \%$ & 4 & 2937 & Hazard Ratio (IV, Random, 95\% Cl) & $0.88[0.78,1.00]$ \\
\hline 1.1.3 PD-L1 TPS $\geq 50 \%$ & 6 & 2111 & Hazard Ratio (IV, Random, 95\% Cl) & $0.68[0.60,0.76]$ \\
\hline $\begin{array}{l}1.2 \text { Overall Survival by Tumor Muta- } \\
\text { tional Burden }\end{array}$ & 4 & 2035 & Hazard Ratio (IV, Random, 95\% Cl) & $0.89[0.76,1.05]$ \\
\hline 1.2.1 Tumor Mutational Burden low & 4 & 1380 & Hazard Ratio (IV, Random, 95\% Cl) & $1.01[0.88,1.15]$ \\
\hline 1.2.2 Tumor Mutational Burden high & 4 & 655 & Hazard Ratio (IV, Random, 95\% Cl) & $0.72[0.57,0.90]$ \\
\hline 1.3 Overall survival by age (PDL1 $\geq 1 \%$ ) & 2 & 1815 & Hazard Ratio (IV, Random, 95\% Cl) & $0.90[0.78,1.06]$ \\
\hline 1.3.1 Age $<65$ years & 2 & 988 & Hazard Ratio (IV, Random, 95\% Cl) & $0.93[0.68,1.29]$ \\
\hline 1.3.2 Age $\geq 65$ years & 2 & 827 & Hazard Ratio (IV, Random, 95\% Cl) & $0.90[0.72,1.13]$ \\
\hline 1.4 Overall survival by age (PDL1 $\geq 50 \%$ ) & 3 & 1006 & Hazard Ratio (IV, Random, 95\% Cl) & $0.67[0.56,0.79]$ \\
\hline 1.4.1 Age $<65$ years & 3 & 571 & Hazard Ratio (IV, Random, 95\% CI) & $0.72[0.57,0.90]$ \\
\hline
\end{tabular}

Single or combined immune checkpoint inhibitors compared to first-line platinum-based chemotherapy with or without bevacizumab for 69 people with advanced non-small cell lung cancer (Review)

Copyright (c) 2020 The Cochrane Collaboration. Published by John Wiley \& Sons, Ltd. 


\begin{tabular}{|c|c|c|c|c|}
\hline Outcome or subgroup title & $\begin{array}{l}\text { No. of } \\
\text { studies }\end{array}$ & $\begin{array}{l}\text { No. of } \\
\text { partici- } \\
\text { pants }\end{array}$ & Statistical method & Effect size \\
\hline 1.4.2 Age $\geq 65$ years & 2 & 435 & Hazard Ratio (IV, Random, 95\% CI) & $0.60[0.47,0.78]$ \\
\hline 1.5 Overall survival by sex (PDL1 $\geq 1 \%$ ) & 2 & 1815 & Hazard Ratio (IV, Random, 95\% CI) & $0.90[0.78,1.03]$ \\
\hline 1.5.1 Male & 2 & 1234 & Hazard Ratio (IV, Random, 95\% CI) & $0.86[0.71,1.03]$ \\
\hline 1.5.2 Female & 2 & 581 & Hazard Ratio (IV, Random, 95\% CI) & $0.98[0.77,1.25]$ \\
\hline 1.6 Overall survival by sex (PDL1 $\geq 50 \%)$ & 3 & 1108 & Hazard Ratio (IV, Random, 95\% Cl) & $0.68[0.58,0.79]$ \\
\hline 1.6.2 Female & 3 & 363 & Hazard Ratio (IV, Random, 95\% Cl) & $0.81[0.61,1.08]$ \\
\hline $\begin{array}{l}1.7 \text { Overall survival by smoking status } \\
\text { (PDL } 1 \geq 1 \%)\end{array}$ & 2 & 1808 & Hazard Ratio (IV, Random, 95\% Cl) & $0.92[0.78,1.10]$ \\
\hline 1.7.1 Never smoked & 2 & 341 & Hazard Ratio (IV, Random, 95\% CI) & $1.00[0.76,1.33]$ \\
\hline 1.7.2 Former smoker & 2 & 1089 & Hazard Ratio (IV, Random, 95\% Cl) & $0.87[0.57,1.33]$ \\
\hline 1.7.3 Current smokers & 2 & 378 & Hazard Ratio (IV, Random, 95\% CI) & $0.98[0.75,1.27]$ \\
\hline $\begin{array}{l}1.8 \text { Overall survival by smoking status } \\
\text { (PDL } 1 \geq 50 \%)\end{array}$ & 3 & 1109 & Hazard Ratio (IV, Random, 95\% Cl) & $0.70[0.56,0.86]$ \\
\hline 1.8.2 Former smoker & 3 & 700 & Hazard Ratio (IV, Random, 95\% CI) & $0.60[0.49,0.73]$ \\
\hline 1.8.3 Current smokers & 3 & 230 & Hazard Ratio (IV, Random, 95\% CI) & $0.65[0.44,0.97]$ \\
\hline 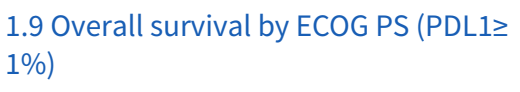 & 2 & 1814 & Hazard Ratio (IV, Random, 95\% CI) & $0.89[0.77,1.03]$ \\
\hline 1.9.1 ECOG PS 0 & 2 & 568 & Hazard Ratio (IV, Random, 95\% Cl) & $0.90[0.63,1.29]$ \\
\hline 1.9.2 ECOG PS 1 & 2 & 1246 & Hazard Ratio (IV, Random, 95\% CI) & $0.90[0.74,1.09]$ \\
\hline $\begin{array}{l}1.10 \text { Overall survival by ECOG PS } \\
\text { (PDL } 1 \geq 50 \%)\end{array}$ & 3 & 1108 & Hazard Ratio (IV, Random, 95\% CI) & $0.65[0.55,0.77]$ \\
\hline 1.10.1 ECOG PS 0 & 3 & 367 & Hazard Ratio (IV, Random, 95\% CI) & $0.60[0.44,0.81]$ \\
\hline 1.10.2 ECOG PS 1 & 3 & 741 & Hazard Ratio (IV, Random, 95\% CI) & $0.67[0.55,0.82]$ \\
\hline $\begin{array}{l}1.11 \text { Overall survival by histological } \\
\text { type (PDL } 1 \geq 1 \% \text { ) }\end{array}$ & 2 & 1815 & Hazard Ratio (IV, Random, 95\% CI) & $0.89[0.73,1.07]$ \\
\hline 1.11.1 Squamous & 2 & 621 & Hazard Ratio (IV, Random, 95\% CI) & $0.76[0.63,0.93]$ \\
\hline 1.11.2 Non-squamous & 2 & 1194 & Hazard Ratio (IV, Random, 95\% CI) & $0.99[0.73,1.33]$ \\
\hline
\end{tabular}




\begin{tabular}{|c|c|c|c|c|}
\hline Outcome or subgroup title & $\begin{array}{l}\text { No. of } \\
\text { studies }\end{array}$ & $\begin{array}{l}\text { No. of } \\
\text { partici- } \\
\text { pants }\end{array}$ & Statistical method & Effect size \\
\hline $\begin{array}{l}1.12 \text { Overall survival by histological } \\
\text { type (PDL } 1 \geq 50 \% \text { ) }\end{array}$ & 3 & 1109 & Hazard Ratio (IV, Random, 95\% CI) & $0.66[0.56,0.77]$ \\
\hline 1.12.1 Squamous & 3 & 327 & Hazard Ratio (IV, Random, 95\% Cl) & $0.57[0.43,0.76]$ \\
\hline 1.12.2 Non-squamous & 3 & 782 & Hazard Ratio (IV, Random, 95\% CI) & $0.69[0.55,0.87]$ \\
\hline $\begin{array}{l}1.13 \text { Progression free survival by PD-L1 } \\
\text { expression }\end{array}$ & 5 & & Hazard Ratio (IV, Random, 95\% Cl) & Subtotals only \\
\hline 1.13.1 PDL $1 \geq 1 \%$ & 3 & 2369 & Hazard Ratio (IV, Random, 95\% Cl) & $0.99[0.79,1.24]$ \\
\hline 1.13.2 PDL1 $1 \geq 50 \%$ & 5 & 1886 & Hazard Ratio (IV, Random, 95\% Cl) & $0.68[0.52,0.88]$ \\
\hline $\begin{array}{l}\text { 1.14 Progression Free Survival by Tu- } \\
\text { mor Mutational Burden }\end{array}$ & 4 & 2035 & Hazard Ratio (IV, Random, 95\% Cl) & $0.97[0.76,1.22]$ \\
\hline 1.14.1 Tumor Mutational Burden high & 4 & 655 & Hazard Ratio (IV, Random, 95\% Cl) & $0.72[0.60,0.86]$ \\
\hline 1.14.2 Tumor Mutational Burden low & 4 & 1380 & Hazard Ratio (IV, Random, 95\% Cl) & $1.24[1.00,1.55]$ \\
\hline $\begin{array}{l}\text { 1.15 Overall response rate by PDL1 ex- } \\
\text { pression }\end{array}$ & 4 & & Risk Ratio (IV, Random, 95\% CI) & Subtotals only \\
\hline 1.15.1 PDL $1 \geq 1 \%$ & 2 & 1828 & Risk Ratio (IV, Random, 95\% Cl) & $0.99[0.86,1.15]$ \\
\hline 1.15.2 PDL $1 \geq 50 \%$ & 4 & 1672 & Risk Ratio (IV, Random, 95\% CI) & $1.40[1.12,1.75]$ \\
\hline $\begin{array}{l}1.16 \text { Overall Response Rate by Tumor } \\
\text { Mutational Burden }\end{array}$ & 3 & 1646 & Risk Ratio (M-H, Random, 95\% Cl) & $0.97[0.73,1.27]$ \\
\hline 1.16.1 Tumor Mutational Burden high & 3 & 599 & Risk Ratio (M-H, Random, 95\% Cl) & $1.25[0.99,1.59]$ \\
\hline 1.16.2 Tumor Mutational Burden low & 3 & 1047 & Risk Ratio (M-H, Random, 95\% Cl) & $0.73[0.59,0.91]$ \\
\hline 1.17 Adverse Events grade 3-5 & 5 & 6692 & Risk Ratio (M-H, Random, 95\% Cl) & $0.43[0.36,0.52]$ \\
\hline 1.17.1 Adverse Events grade 3-4 & 5 & 3346 & Risk Ratio (M-H, Random, 95\% Cl) & $0.41[0.33,0.50]$ \\
\hline $\begin{array}{l}\text { 1.17.2 Adverse Events grade } 5 \text { (toxic } \\
\text { deaths) }\end{array}$ & 5 & 3346 & Risk Ratio (M-H, Random, 95\% Cl) & $0.78[0.43,1.41]$ \\
\hline $\begin{array}{l}1.18 \mathrm{QOL}-\mathrm{C} 30 \mathrm{GHS} / \mathrm{QOL} \text { - change from } \\
\text { baseline to week } 15\end{array}$ & 1 & & $\begin{array}{l}\text { Mean Difference (IV, Random, 95\% } \\
\mathrm{CI})\end{array}$ & Subtotals only \\
\hline 1.19 Time to deterioration - QLQ-LC13 & 1 & & Hazard Ratio (IV, Random, 95\% Cl) & Totals not selected \\
\hline 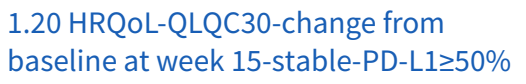 & 1 & & Risk Ratio (M-H, Random, 95\% Cl) & Subtotals only \\
\hline 1.20.1 GHS/QOL & 1 & 297 & Risk Ratio (M-H, Random, 95\% Cl) & $0.89[0.68,1.17]$ \\
\hline 1.20.2 Physical functioning & 1 & 297 & Risk Ratio (M-H, Random, 95\% Cl) & $1.35[1.05,1.74]$ \\
\hline
\end{tabular}

Single or combined immune checkpoint inhibitors compared to first-line platinum-based chemotherapy with or without bevacizumab for 


\begin{tabular}{|c|c|c|c|c|}
\hline Outcome or subgroup title & $\begin{array}{l}\text { No. of } \\
\text { studies }\end{array}$ & $\begin{array}{l}\text { No. of } \\
\text { partici- } \\
\text { pants }\end{array}$ & Statistical method & Effect size \\
\hline 1.20.3 Role functioning & 1 & 297 & Risk Ratio (M-H, Random, 95\% Cl) & $1.16[0.88,1.55]$ \\
\hline 1.20.4 Emotional functioning & 1 & 297 & Risk Ratio (M-H, Random, 95\% Cl) & $1.11[0.88,1.39]$ \\
\hline 1.20.5 Cognitive functioning & 1 & 297 & Risk Ratio (M-H, Random, 95\% Cl) & $1.12[0.89,1.40]$ \\
\hline 1.20.6 Social functioning & 1 & 297 & Risk Ratio (M-H, Random, 95\% Cl) & $1.21[0.92,1.58]$ \\
\hline 1.20.7 Fatigue & 1 & 297 & Risk Ratio (M-H, Random, 95\% Cl) & $1.19[0.82,1.71]$ \\
\hline 1.20.8 Nausea and vomiting & 1 & 297 & Risk Ratio (M-H, Random, 95\% Cl) & $1.24[1.05,1.47]$ \\
\hline 1.20.9 Pain & 1 & 297 & Risk Ratio (M-H, Random, 95\% Cl) & $0.82[0.62,1.10]$ \\
\hline 1.20.10 Dyspnoea & 1 & 297 & Risk Ratio (M-H, Random, 95\% Cl) & $1.28[0.99,1.64]$ \\
\hline 1.20.11 Insomnia & 1 & 297 & Risk Ratio (M-H, Random, 95\% Cl) & $1.36[1.03,1.79]$ \\
\hline 1.20.12 Appetite loss & 1 & 297 & Risk Ratio (M-H, Random, 95\% Cl) & $1.16[0.90,1.49]$ \\
\hline 1.20.13 Constipation & 1 & 297 & Risk Ratio (M-H, Random, 95\% Cl) & $1.09[0.89,1.33]$ \\
\hline 1.20.14 Diarrhoea & 1 & 297 & Risk Ratio (M-H, Random, 95\% Cl) & $1.21[1.04,1.40]$ \\
\hline 1.20.15 Financial difficulties & 1 & 297 & Risk Ratio (M-H, Random, 95\% Cl) & $1.16[0.96,1.41]$ \\
\hline $\begin{array}{l}1.21 \mathrm{HRQ} \text { LL-QLQC30-change from } \\
\text { baseline at week } 15 \text {-deterioration - } \\
\text { PDL1 } \geq 50 \%\end{array}$ & 1 & & Risk Ratio (M-H, Random, 95\% Cl) & Subtotals only \\
\hline 1.21.1 GHS/QOL & 1 & 297 & Risk Ratio (M-H, Random, 95\% Cl) & $0.71[0.48,1.06]$ \\
\hline 1.21.2 Physical functioning & 1 & 297 & Risk Ratio (M-H, Random, 95\% Cl) & $0.55[0.38,0.82]$ \\
\hline 1.21.3 Role functioning & 1 & 297 & Risk Ratio (M-H, Random, 95\% Cl) & $0.61[0.42,0.88]$ \\
\hline 1.21.4 Emotional functioning & 1 & 297 & Risk Ratio (M-H, Random, 95\% Cl) & $1.20[0.67,2.14]$ \\
\hline 1.21.5 Cognitive functioning & 1 & 297 & Risk Ratio (M-H, Random, 95\% Cl) & $1.03[0.70,1.51]$ \\
\hline 1.21.6 Social functioning & 1 & 297 & Risk Ratio (M-H, Random, 95\% Cl) & $0.61[0.41,0.89]$ \\
\hline 1.21.7 Fatigue & 1 & 297 & Risk Ratio (M-H, Random, 95\% Cl) & $0.43[0.29,0.63]$ \\
\hline 1.21.8 Nausea and vomiting & 1 & 297 & Risk Ratio (M-H, Random, 95\% Cl) & $0.48[0.28,0.81]$ \\
\hline 1.21.9 Pain & 1 & 297 & Risk Ratio (M-H, Random, 95\% Cl) & $0.75[0.47,1.18]$ \\
\hline 1.21.10 Dyspnoea & 1 & 297 & Risk Ratio (M-H, Random, 95\% Cl) & $0.43[0.27,0.68]$ \\
\hline 1.21.11 Insomnia & 1 & 297 & Risk Ratio (M-H, Random, 95\% Cl) & $0.68[0.44,1.05]$ \\
\hline 1.21.12 Appetite loss & 1 & 297 & Risk Ratio (M-H, Random, 95\% Cl) & $0.59[0.37,0.94]$ \\
\hline
\end{tabular}

Single or combined immune checkpoint inhibitors compared to first-line platinum-based chemotherapy with or without bevacizumab for 


\begin{tabular}{|c|c|c|c|c|}
\hline Outcome or subgroup title & $\begin{array}{l}\text { No. of } \\
\text { studies }\end{array}$ & $\begin{array}{l}\text { No. of } \\
\text { partici- } \\
\text { pants }\end{array}$ & Statistical method & Effect size \\
\hline 1.21.13 Constipation & 1 & 297 & Risk Ratio (M-H, Random, 95\% Cl) & $0.63[0.41,0.97]$ \\
\hline 1.21.14 Diarrhea & 1 & 297 & Risk Ratio (M-H, Random, 95\% Cl) & $0.74[0.44,1.23]$ \\
\hline 1.21.15 Financial difficulties & 1 & 297 & Risk Ratio (M-H, Random, 95\% Cl) & $0.75[0.49,1.15]$ \\
\hline $\begin{array}{l}1.22 \mathrm{HRQOL}-\mathrm{Q} L Q C 30 \text {-change from } \\
\text { baseline at week } 15 \text {-improvement - } \\
\text { PD-L1 } 50 \%\end{array}$ & 1 & & Risk Ratio (M-H, Random, 95\% Cl) & Subtotals only \\
\hline 1.22.1 GHS/QOL & 1 & 297 & Risk Ratio (M-H, Random, 95\% Cl) & $1.51[1.08,2.10]$ \\
\hline 1.22.2 Physical functioning & 1 & 297 & Risk Ratio (M-H, Random, 95\% Cl) & $1.09[0.74,1.61]$ \\
\hline 1.22.3 Role functioning & 1 & 297 & Risk Ratio (M-H, Random, 95\% Cl) & $1.29[0.92,1.81]$ \\
\hline 1.22.4 Emotional functioning & 1 & 297 & Risk Ratio (M-H, Random, 95\% Cl) & $0.81[0.60,1.10]$ \\
\hline 1.22.5 Cognitive functioning & 1 & 297 & Risk Ratio (M-H, Random, 95\% Cl) & $0.75[0.50,1.15]$ \\
\hline 1.22.6 Social functioning & 1 & 297 & Risk Ratio (M-H, Random, 95\% Cl) & $1.20[0.85,1.69]$ \\
\hline 1.22.7 Fatigue & 1 & 297 & Risk Ratio (M-H, Random, 95\% Cl) & $1.61[1.21,2.13]$ \\
\hline 1.22.8 Nausea and vomiting & 1 & 297 & Risk Ratio (M-H, Random, 95\% Cl) & $0.90[0.55,1.50]$ \\
\hline 1.22.9 Pain & 1 & 297 & Risk Ratio (M-H, Random, 95\% Cl) & $1.39[1.05,1.84]$ \\
\hline 1.22.10 Dyspnoea & 1 & 297 & Risk Ratio (M-H, Random, 95\% Cl) & $1.24[0.88,1.73]$ \\
\hline 1.22.11 Insomnia & 1 & 297 & Risk Ratio (M-H, Random, 95\% Cl) & $0.89[0.66,1.21]$ \\
\hline 1.22.12 Appetite loss & 1 & 297 & Risk Ratio (M-H, Random, 95\% Cl) & $1.12[0.82,1.54]$ \\
\hline 1.22.13 Constipation & 1 & 297 & Risk Ratio (M-H, Random, 95\% Cl) & $1.33[0.84,2.12]$ \\
\hline 1.22.14 Diarrhea & 1 & 297 & Risk Ratio (M-H, Random, 95\% Cl) & $0.49[0.25,0.94]$ \\
\hline 1.22.15 Financial difficulties & 1 & 297 & Risk Ratio (M-H, Random, 95\% Cl) & $0.88[0.54,1.42]$ \\
\hline
\end{tabular}




\section{Analysis 1.1. Comparison 1: Single immunecheckpoint inhibitors vs chemotherapy, Outcome 1: Overall survival by PD-L1 expression}

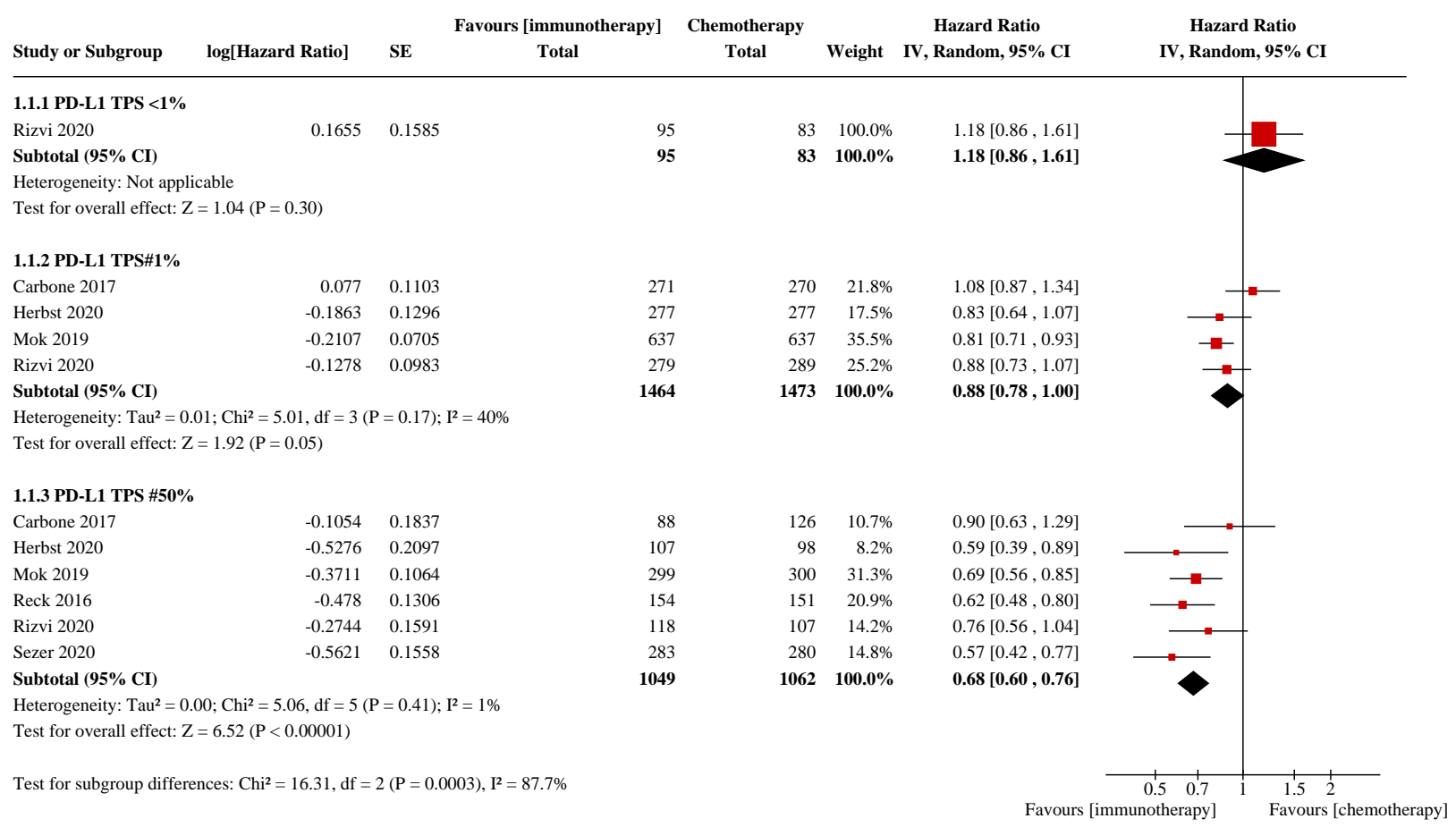

Analysis 1.2. Comparison 1: Single immunecheckpoint inhibitors vs chemotherapy, Outcome 2: Overall Survival by Tumor Mutational Burden

$\begin{array}{lccccccc} & & \text { Immunotherapy } & \text { Chemotherapy } & & \text { Hazard Ratio } & \text { Hazard Ratio } \\ \text { Study or Subgroup } & \log [\text { Hazard Ratio] } & \text { SE } & \text { Total } & \text { Total } & \text { Weight } & \text { IV, Random, 95\% CI } & \text { IV, Random, 95\% CI }\end{array}$

\begin{tabular}{lrlrrrr}
\hline 1.2.1 Tumor Mutational Burden low & & & & & & \\
Carbone 2017 & -0.0101 & 0.1768 & 111 & 94 & $12.2 \%$ & $0.99[0.70,1.40]$ \\
Herbst 2020 & 0.0198 & 0.1579 & 333 & 0 & $13.7 \%$ & $1.02[0.75,1.39]$ \\
Mok 2019 & 0.0862 & 0.1129 & 234 & 214 & $18.0 \%$ & $1.09[0.87,1.36]$ \\
Rizvi 2020 & -0.0726 & 0.1128 & 209 & 185 & $18.0 \%$ & $0.93[0.75,1.16]$ \\
Subtotal (95\% CI) & & & $\mathbf{8 8 7}$ & $\mathbf{4 9 3}$ & $\mathbf{6 1 . 9 \%}$ & $\mathbf{1 . 0 1}[\mathbf{0 . 8 8 , \mathbf { 1 . 1 5 }}$
\end{tabular}

Heterogeneity: $\mathrm{Tau}^{2}=0.00 ; \mathrm{Chi}^{2}=1.01, \mathrm{df}=3(\mathrm{P}=0.80) ; \mathrm{I}^{2}=0 \%$

Test for overall effect: $\mathrm{Z}=0.10(\mathrm{P}=0.92)$

1.2.2 Tumor Mutational Burden high

$\begin{array}{lrr}\text { Carbone } 2017 & 0.0953 & 0.2735 \\ \text { Herbst } 2020 & -0.2614 & 0.3858 \\ \text { Mok 2019 } & -0.478 & 0.13 \\ \text { Rizvi } 2020 & -0.3285 & 0.1925\end{array}$

Rizvi 2020 $-0.3285 \quad 0.1925$

Subtotal $(\mathbf{9 5 \%}$ CI $)$

Heterogeneity: $\mathrm{Tau}^{2}=0.01 ; \mathrm{Chi}^{2}=3.68, \mathrm{df}=3(\mathrm{P}=0.30) ; \mathrm{I}^{2}=19 \%$

Test for overall effect: $\mathrm{Z}=2.86(\mathrm{P}=0.004)$

Total (95\% CI)

Heterogeneity: $\mathrm{Tau}^{2}=0.03 ; \mathrm{Chi}^{2}=14.14, \mathrm{df}=7(\mathrm{P}=0.05) ; \mathrm{I}^{2}=51 \%$

Test for overall effect: $\mathrm{Z}=1.37(\mathrm{P}=0.17)$

Test for subgroup differences: $\mathrm{Ch}^{2}=6.39, \mathrm{df}=1(\mathrm{P}=0.01), \mathrm{I}^{2}=84.4 \%$

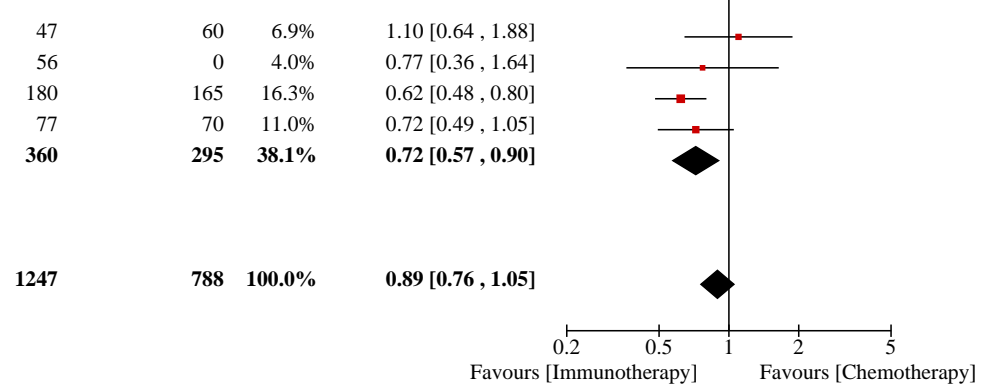

Single or combined immune checkpoint inhibitors compared to first-line platinum-based chemotherapy with or without bevacizumab for 


\section{Analysis 1.3. Comparison 1: Single immunecheckpoint inhibitors vs chemotherapy, Outcome 3: Overall survival by age (PDL1 1\%)}

\begin{tabular}{|c|c|c|c|c|c|c|c|}
\hline Study or Subgroup & $\log [$ Hazard Ratio] & SE & $\begin{array}{c}\text { Immunotherapy } \\
\text { Total }\end{array}$ & $\begin{array}{c}\text { Chemotherapy } \\
\text { Total }\end{array}$ & Weight & $\begin{array}{c}\text { Hazard Ratio } \\
\text { IV, Random, 95\% CI }\end{array}$ & $\begin{array}{c}\text { Hazard Ratio } \\
\text { IV, Random, 95\% CI }\end{array}$ \\
\hline \multicolumn{8}{|l|}{ 1.3.1 Age $<65$ years } \\
\hline Carbone 2017 & 0.1222 & 0.1579 & 148 & 133 & $18.2 \%$ & $1.13[0.83,1.54]$ & $=$ \\
\hline Mok 2019 & -0.2107 & 0.0972 & 359 & 348 & $33.1 \%$ & $0.81[0.67,0.98]$ & \\
\hline Subtotal $(95 \%$ CI $)$ & & & 507 & 481 & $51.3 \%$ & $0.93[0.68,1.29]$ & \\
\hline \multicolumn{8}{|c|}{ Heterogeneity: $\mathrm{Tau}^{2}=0.04 ; \mathrm{Chi}^{2}=3.22, \mathrm{df}=1(\mathrm{P}=0.07) ; \mathrm{I}^{2}=69 \%$} \\
\hline \multicolumn{8}{|c|}{ Test for overall effect: $\mathrm{Z}=0.41(\mathrm{P}=0.68)$} \\
\hline \multicolumn{8}{|l|}{ 1.3.2 Age \#65 years } \\
\hline Carbone 2017 & 0.0392 & 0.1553 & 123 & 137 & $18.6 \%$ & $1.04[0.77,1.41]$ & - \\
\hline Mok 2019 & -0.1985 & 0.1063 & 278 & 289 & $30.1 \%$ & $0.82[0.67,1.01]$ & \\
\hline Subtotal $(95 \%$ CI $)$ & & & 401 & 426 & $48.7 \%$ & $0.90[0.72,1.13]$ & \\
\hline \multicolumn{8}{|c|}{ Heterogeneity: $\mathrm{Tau}^{2}=0.01 ; \mathrm{Chi}^{2}=1.60, \mathrm{df}=1(\mathrm{P}=0.21) ; \mathrm{I}^{2}=37 \%$} \\
\hline \multicolumn{8}{|c|}{ Test for overall effect: $Z=0.92(P=0.36)$} \\
\hline Total $(95 \%$ CI $)$ & & & 908 & 907 & $100.0 \%$ & $0.90[0.78,1.06]$ & \\
\hline \multicolumn{8}{|c|}{ Heterogeneity: $\mathrm{Tau}^{2}=0.01 ; \mathrm{Chi}^{2}=4.82, \mathrm{df}=3(\mathrm{P}=0.19) ; \mathrm{I}^{2}=38 \%$} \\
\hline \multicolumn{7}{|c|}{ Test for overall effect: $\mathrm{Z}=1.27(\mathrm{P}=0.20)$} & $\begin{array}{lllll}1 & 1 & 1 & 1 & 1 \\
0.5 & 0.7 & 1 & 1.5 & 2\end{array}$ \\
\hline Test for subgroup dif & ences: $\mathrm{Chi}^{2}=0.04, \mathrm{df}=$ & $(\mathrm{P}=0.85$ & $5), I^{2}=0 \%$ & & & ravour & otherapy] \\
\hline
\end{tabular}

Analysis 1.4. Comparison 1: Single immunecheckpoint inhibitors vs chemotherapy, Outcome 4: Overall survival by age (PDL1 $\geq 50 \%$ )

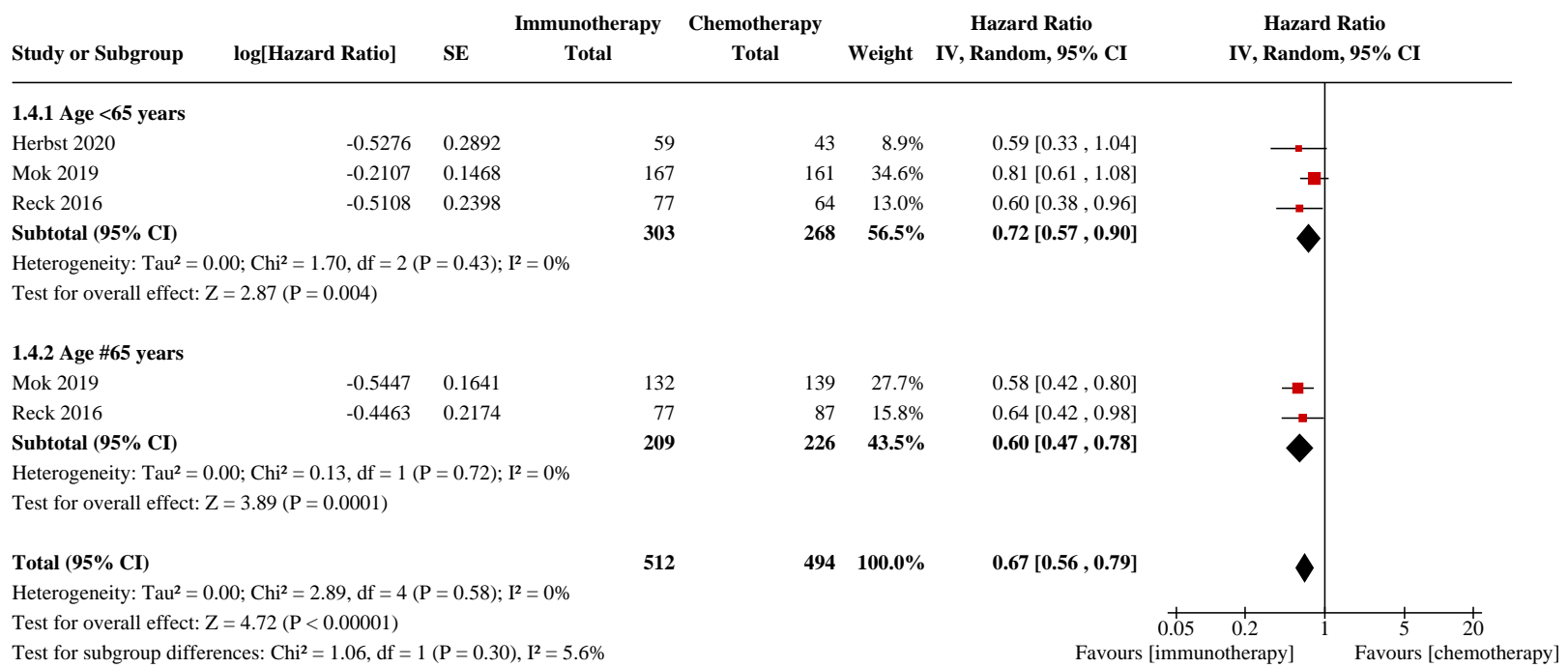


Analysis 1.5. Comparison 1: Single immunecheckpoint inhibitors vs chemotherapy, Outcome 5: Overall survival by sex (PDL1 $\geq 1 \%$ )

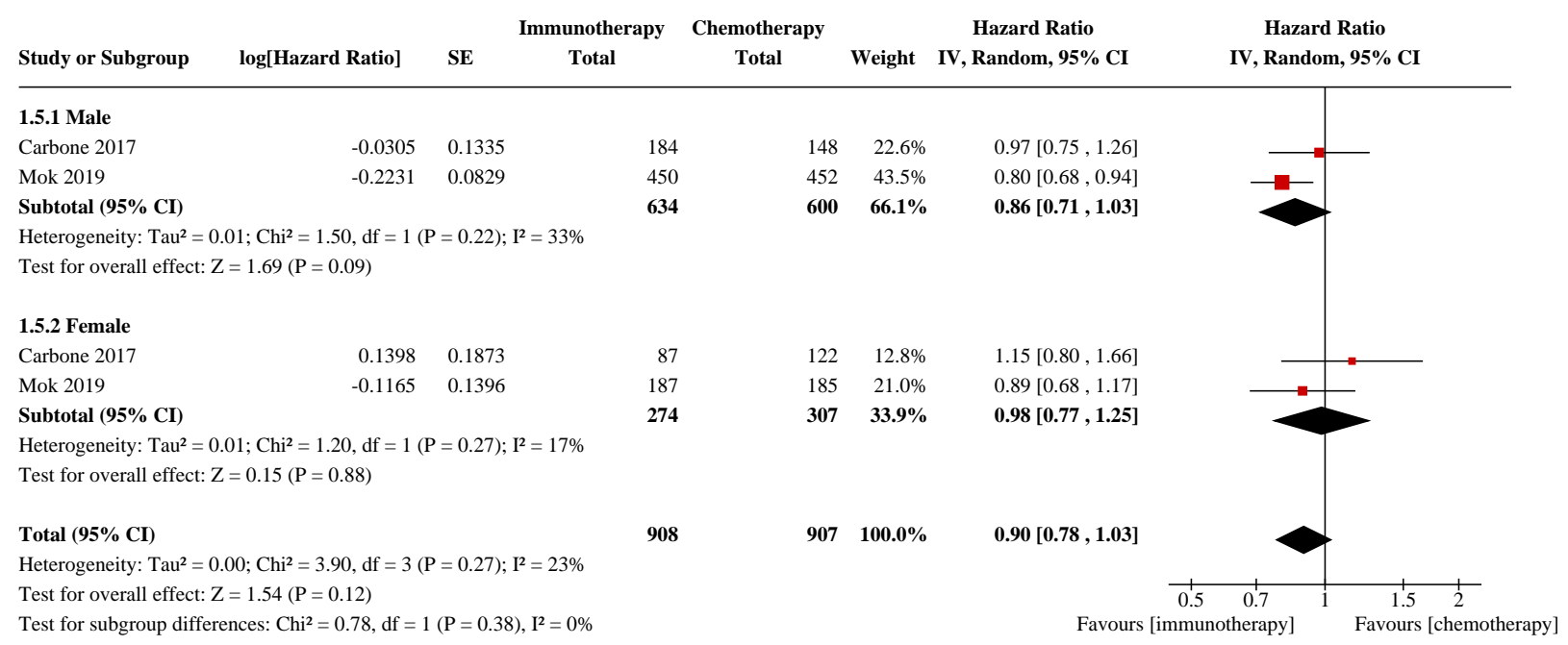

Analysis 1.6. Comparison 1: Single immunecheckpoint inhibitors vs chemotherapy, Outcome 6: Overall survival by sex (PDL1 $\geq 50 \%$ )

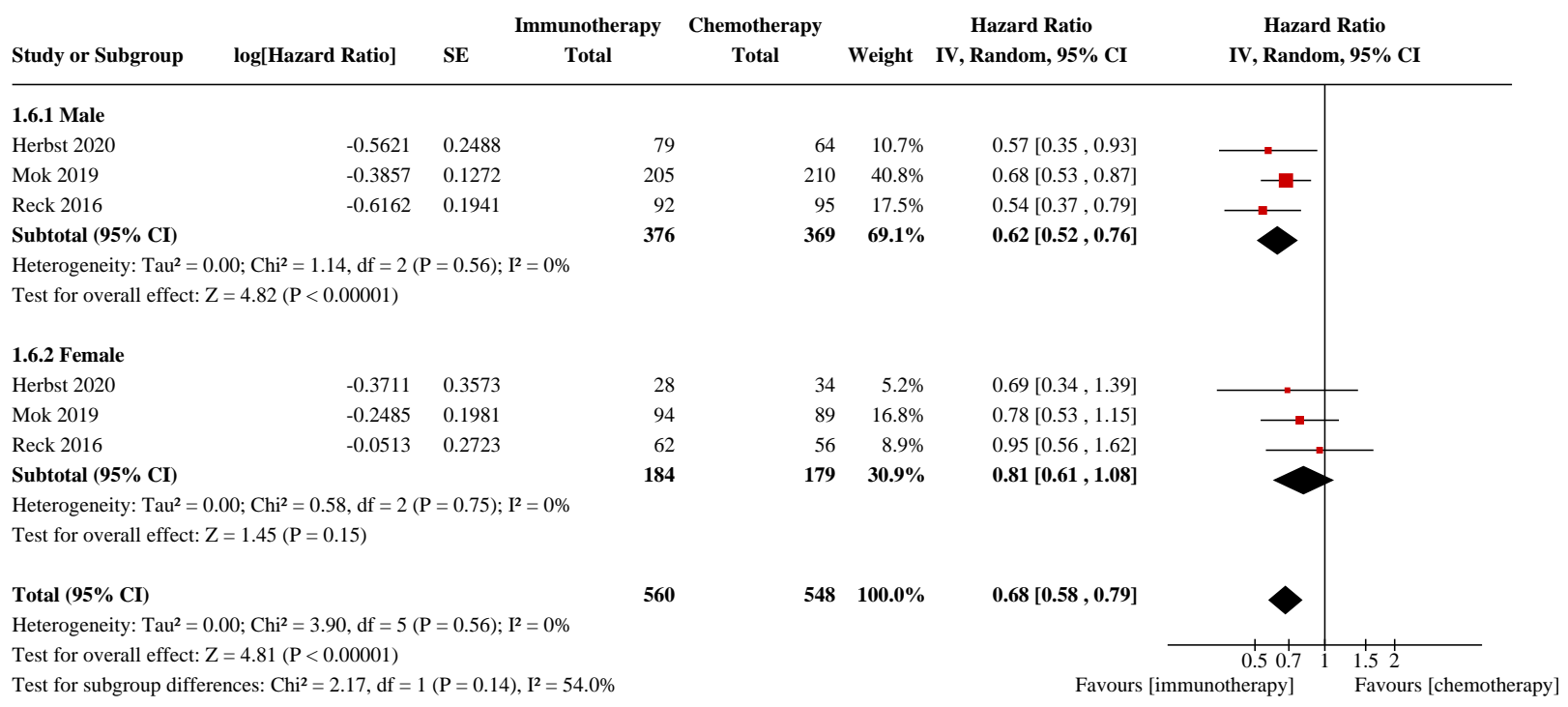


Analysis 1.7. Comparison 1: Single immunecheckpoint inhibitors vs chemotherapy, Outcome 7: Overall survival by smoking status (PDL1 $\geq 1 \%$ )

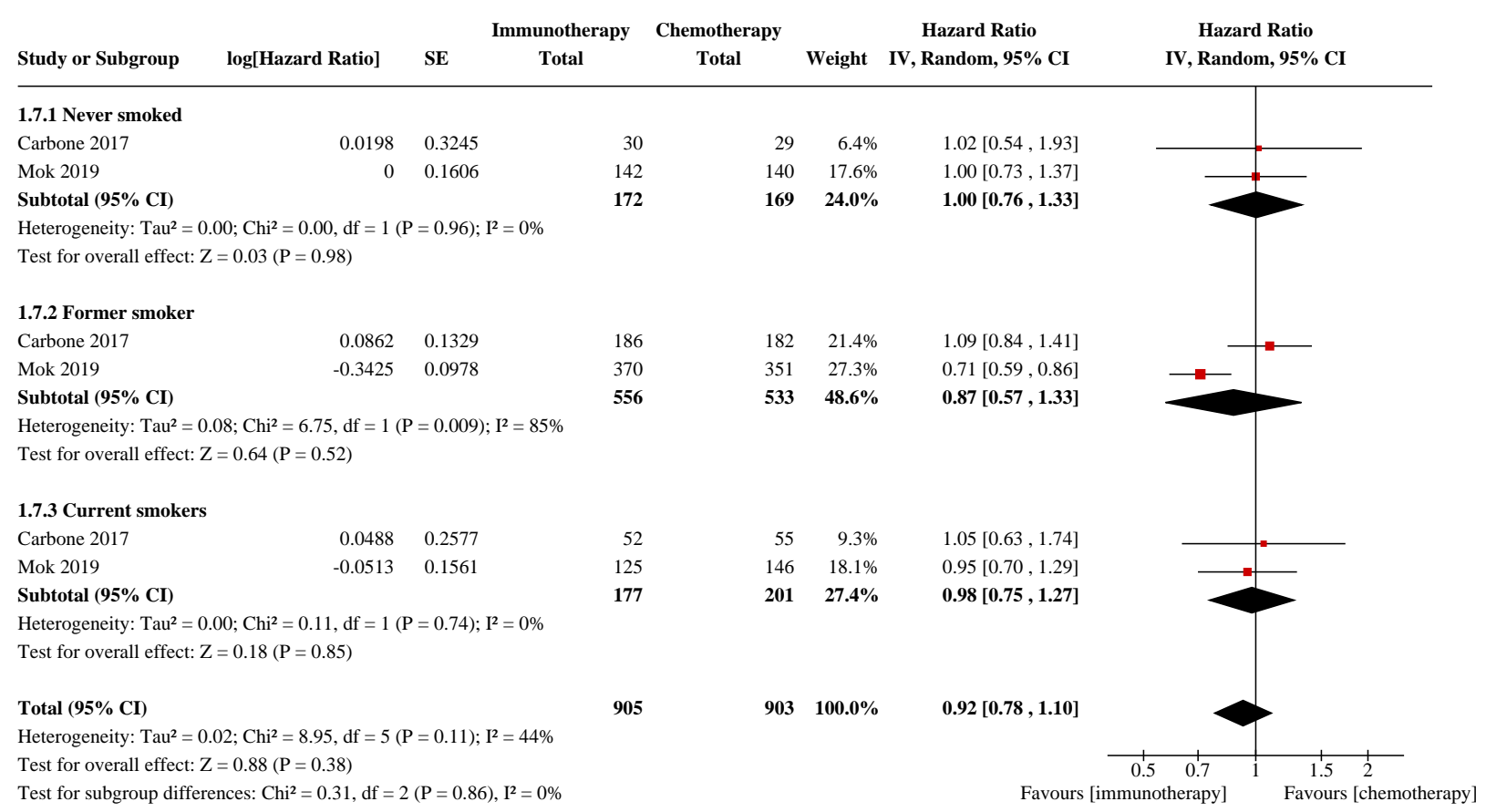

Analysis 1.8. Comparison 1: Single immunecheckpoint inhibitors vs chemotherapy, Outcome 8: Overall survival by smoking status (PDL1 $\mathbf{5 0 \% \text { ) }}$

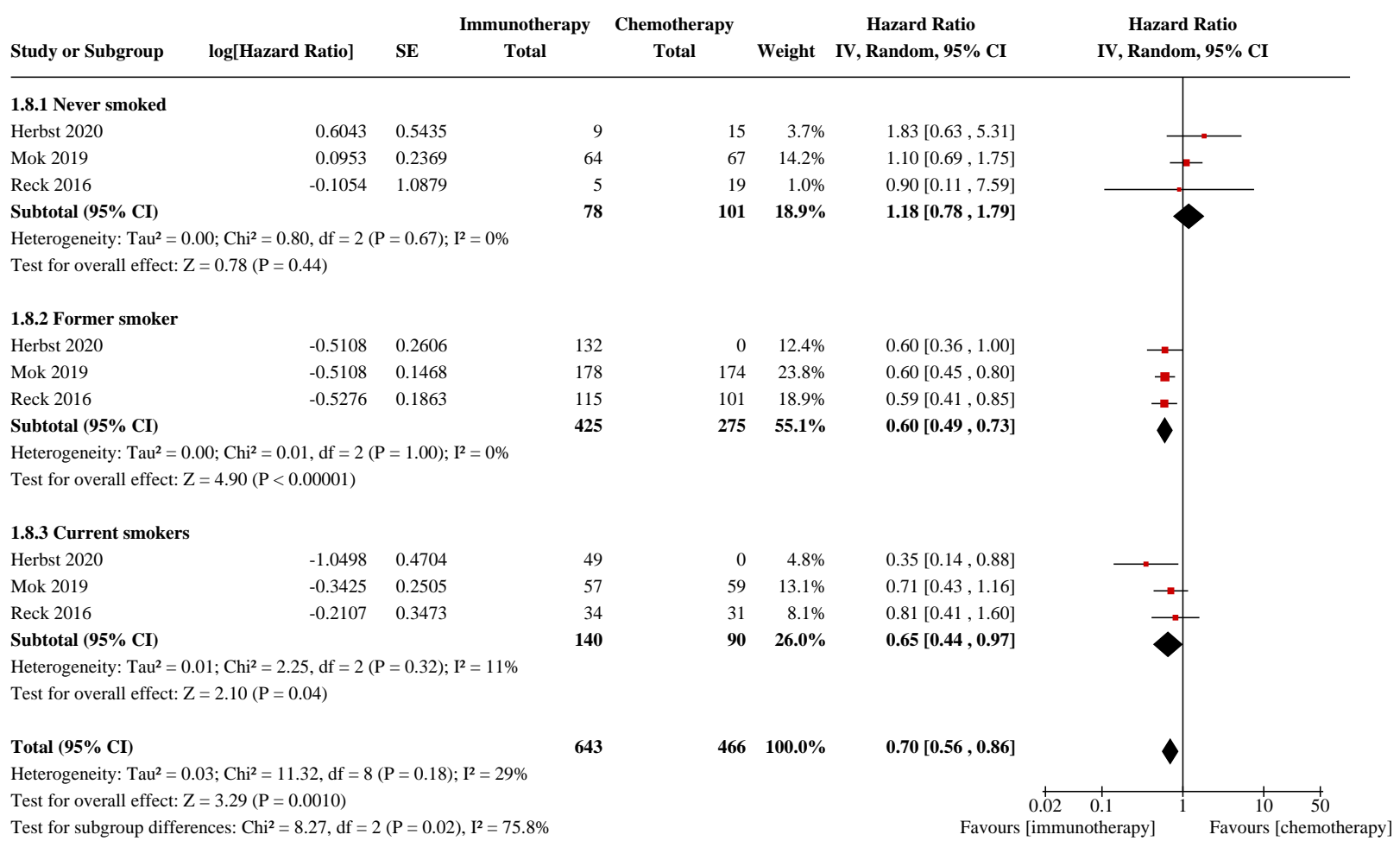


Analysis 1.9. Comparison 1: Single immunecheckpoint inhibitors vs chemotherapy, Outcome 9: Overall survival by ECOG PS (PDL1 $1 \%$ )

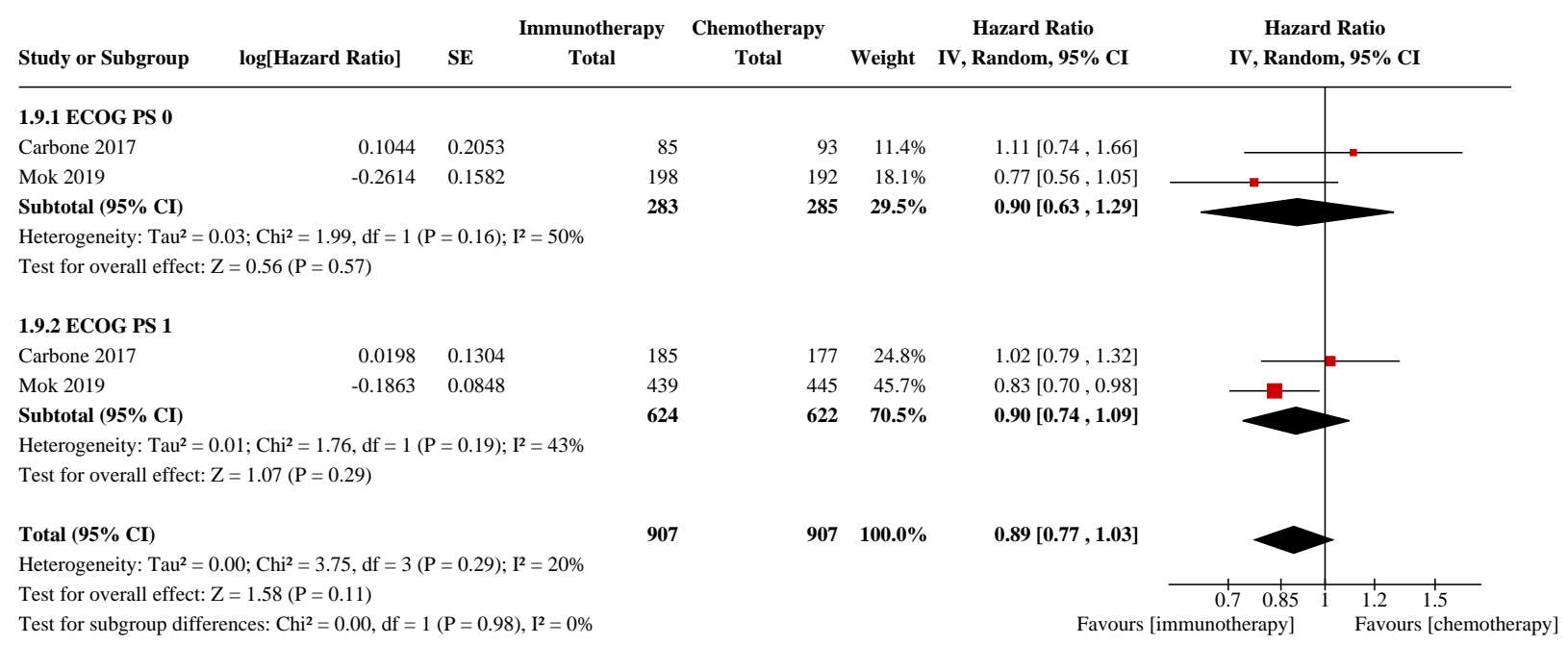

Analysis 1.10. Comparison 1: Single immunecheckpoint inhibitors vs chemotherapy, Outcome 10: Overall survival by ECOG PS (PDL1 $\geq 50 \%$ )

\begin{tabular}{|c|c|c|c|c|c|c|c|c|}
\hline Study or Subgroup & $\log [$ Hazard Ratio] & SE & $\begin{array}{c}\text { Immunotherapy } \\
\text { Total }\end{array}$ & $\begin{array}{c}\text { Chemotherapy } \\
\text { Total }\end{array}$ & Weight & $\begin{array}{c}\text { Hazard Ratio } \\
\text { IV, Random, 95\% CI }\end{array}$ & $\begin{array}{r}\text { Hazard } \\
\text { IV, Randon }\end{array}$ & $\begin{array}{l}\text { Ratio } \\
\text {, } 95 \% \text { CI }\end{array}$ \\
\hline \multicolumn{9}{|l|}{ 1.10.1 ECOG PS 0} \\
\hline Herbst 2020 & -0.8675 & 0.4001 & 35 & 38 & $4.6 \%$ & $0.42[0.19,0.92]$ & $\longleftarrow$ & \\
\hline Mok 2019 & -0.5621 & 0.2098 & 96 & 91 & $16.8 \%$ & $0.57[0.38,0.86]$ & & \\
\hline Reck 2016 & -0.2485 & 0.2874 & 54 & 53 & $8.9 \%$ & $0.78[0.44,1.37]$ & & - \\
\hline Subtotal $(95 \%$ CI $)$ & & & 185 & 182 & $30.3 \%$ & $0.60[0.44,0.81]$ & & \\
\hline \multicolumn{9}{|c|}{ Heterogeneity: $\mathrm{Tau}^{2}=0.00 ; \mathrm{Chi}^{2}=1.69, \mathrm{df}=2(\mathrm{P}=0.43) ; \mathrm{I}^{2}=0 \%$} \\
\hline \multicolumn{9}{|l|}{ 1.10.2 ECOG PS 1} \\
\hline Herbst 2020 & -0.3711 & 0.238 & 72 & 60 & $13.0 \%$ & $0.69[0.43,1.10]$ & & \\
\hline Mok 2019 & -0.3011 & 0.1433 & 203 & 209 & $35.9 \%$ & $0.74[0.56,0.98]$ & & \\
\hline Reck 2016 & -0.5798 & 0.1883 & 99 & 98 & $20.8 \%$ & $0.56[0.39,0.81]$ & & \\
\hline Subtotal $(95 \%$ CI $)$ & & & 374 & 367 & $69.7 \%$ & $0.67[0.55,0.82]$ & & \\
\hline \multicolumn{9}{|c|}{ Heterogeneity: $\mathrm{Tau}^{2}=0.00 ; \mathrm{Chi}^{2}=1.40, \mathrm{df}=2(\mathrm{P}=0.50) ; \mathrm{I}^{2}=0 \%$} \\
\hline Total $(95 \%$ CI $)$ & & & 559 & 549 & $100.0 \%$ & $0.65[0.55,0.77]$ & & \\
\hline \multicolumn{9}{|c|}{ Heterogeneity: $\mathrm{Tau}^{2}=0.00 ; \mathrm{Chi}^{2}=3.49, \mathrm{df}=5(\mathrm{P}=0.62) ; \mathrm{I}^{2}=0 \%$} \\
\hline \multicolumn{6}{|c|}{ Test for overall effect: $\mathrm{Z}=5.05(\mathrm{P}<0.00001)$} & & $\begin{array}{ccc}1 & 1 & 1 \\
0.5 & 0.7 & 1\end{array}$ & 1.52 \\
\hline Test for subgroup diff & nces: $\mathrm{Chi}^{2}=0.40, \mathrm{df}=1$ & $(\mathrm{P}=0.52$ & $\mathrm{I}^{2}=0 \%$ & & & Favours & immunotherapy] & Favours \\
\hline
\end{tabular}


Analysis 1.11. Comparison 1: Single immunecheckpoint inhibitors vs chemotherapy, Outcome 11: Overall survival by histological type (PDL1 $\geq 1 \%$ )

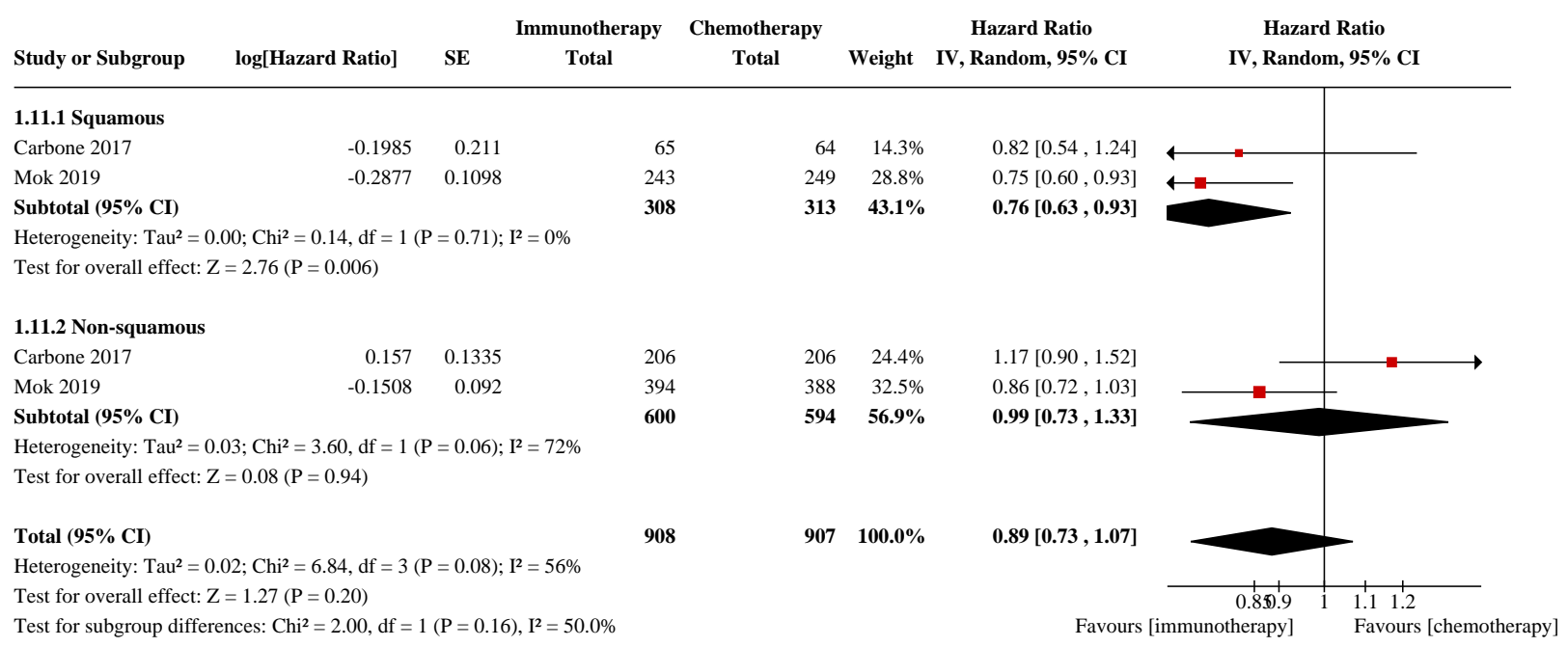

Analysis 1.12. Comparison 1: Single immunecheckpoint inhibitors vs chemotherapy, Outcome 12: Overall survival by histological type (PDL1 $\geq 50 \%$ )

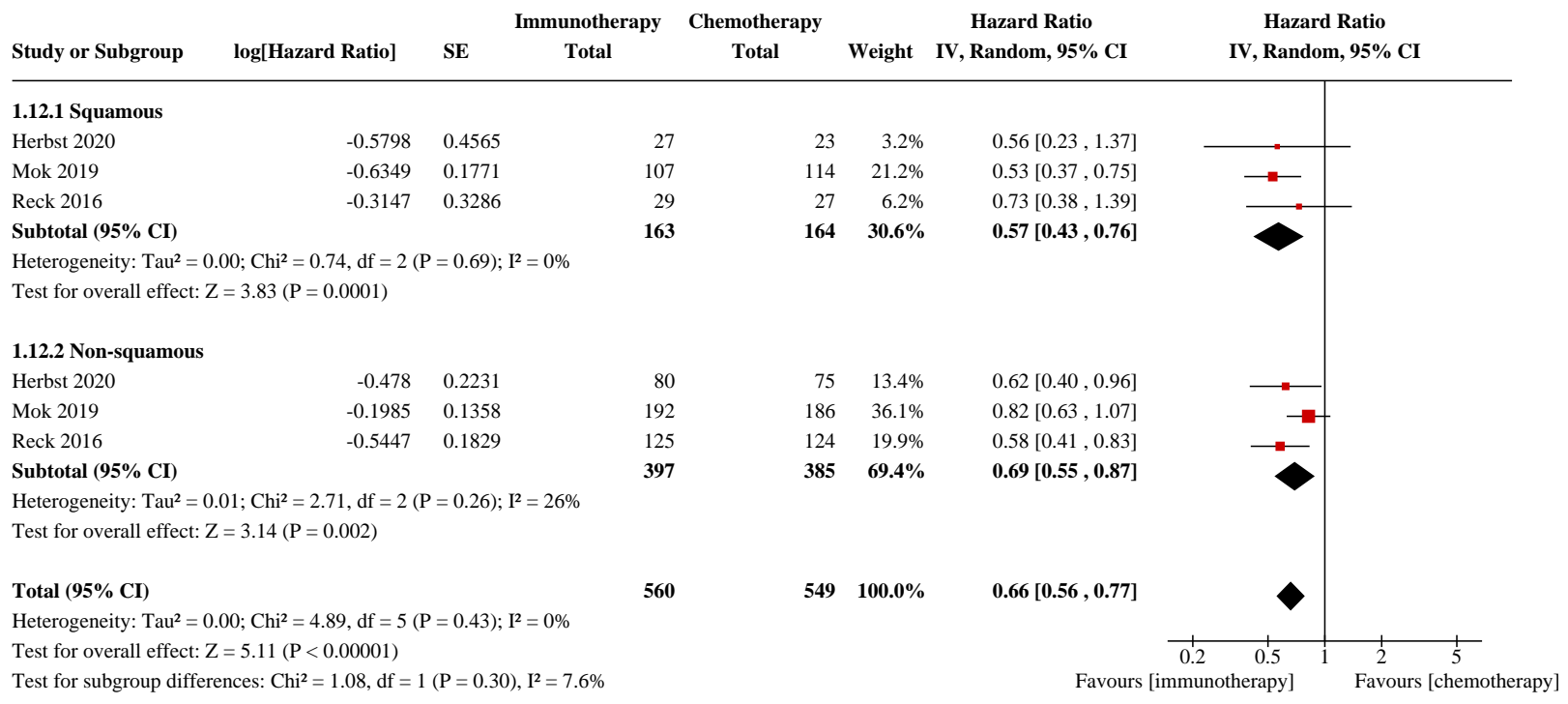


Analysis 1.13. Comparison 1: Single immunecheckpoint inhibitors vs chemotherapy, Outcome 13: Progression free survival by PD-L1 expression

\begin{tabular}{|c|c|c|c|c|c|c|c|}
\hline Study or Subgroup & $\log [$ Hazard Ratio] & SE & $\begin{array}{c}\text { Immunotherapy } \\
\text { Total }\end{array}$ & $\begin{array}{c}\text { Chemotherapy } \\
\text { Total }\end{array}$ & Weight & $\begin{array}{c}\text { Hazard Ratio } \\
\text { IV, Random, 95\% CI }\end{array}$ & $\begin{array}{c}\text { Hazard Ratio } \\
\text { IV, Random, 95\% CI }\end{array}$ \\
\hline \multicolumn{8}{|l|}{ 1.13.1 PDL1\#1\% } \\
\hline Carbone 2017 & 0.157 & 0.1024 & 271 & 270 & $31.3 \%$ & $1.17[0.96,1.43]$ & - \\
\hline Herbst 2020 & -0.2614 & 0.1018 & 277 & 277 & $31.4 \%$ & $0.77[0.63,0.94]$ & - \\
\hline Mok 2019 & 0.0677 & 0.0627 & 637 & 637 & $37.2 \%$ & $1.07[0.95,1.21]$ & \\
\hline Subtotal $(95 \%$ CI) & & & 1185 & 1184 & $100.0 \%$ & $0.99[0.79,1.24]$ & \\
\hline \multicolumn{8}{|c|}{ Heterogeneity: $\mathrm{Tau}^{2}=0.03 ; \mathrm{Chi}^{2}=10.00, \mathrm{df}=2(\mathrm{P}=0.007) ; \mathrm{I}^{2}=80 \%$} \\
\hline \multicolumn{8}{|l|}{ 1.13.2 PDL1\#50\% } \\
\hline Carbone 2017 & 0.0677 & 0.1689 & 88 & 126 & $18.0 \%$ & $1.07[0.77,1.49]$ &. \\
\hline Herbst 2020 & -0.462 & 0.1705 & 107 & 98 & $17.9 \%$ & $0.63[0.45,0.88]$ & - \\
\hline Mok 2019 & -0.2107 & 0.1024 & 299 & 300 & $22.1 \%$ & $0.81[0.66,0.99]$ & \\
\hline Reck 2016 & -0.6931 & 0.1268 & 154 & 151 & $20.7 \%$ & $0.50[0.39,0.64]$ & $\longrightarrow$ \\
\hline Sezer 2020 & -0.6162 & 0.1176 & 283 & 280 & $21.2 \%$ & $0.54[0.43,0.68]$ & $\longrightarrow$ \\
\hline Subtotal $(95 \% \mathrm{CI})$ & & & 931 & 955 & $100.0 \%$ & $0.68[0.52,0.88]$ & \\
\hline \multicolumn{8}{|c|}{ Heterogeneity: $\mathrm{Tau}^{2}=0.07 ; \mathrm{Chi}^{2}=19.93, \mathrm{df}=4(\mathrm{P}=0.0005) ; \mathrm{I}^{2}=80 \%$} \\
\hline \multicolumn{8}{|c|}{ Test for overall effect: $\mathrm{Z}=2.95(\mathrm{P}=0.003)$} \\
\hline \multicolumn{7}{|c|}{ Test for subgroup differences: $\mathrm{Chi}^{2}=4.82, \mathrm{df}=1(\mathrm{P}=0.03), \mathrm{I}^{2}=79.2 \%$} & 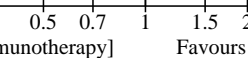 \\
\hline
\end{tabular}

Analysis 1.14. Comparison 1: Single immunecheckpoint inhibitors vs chemotherapy, Outcome 14: Progression Free Survival by Tumor Mutational Burden

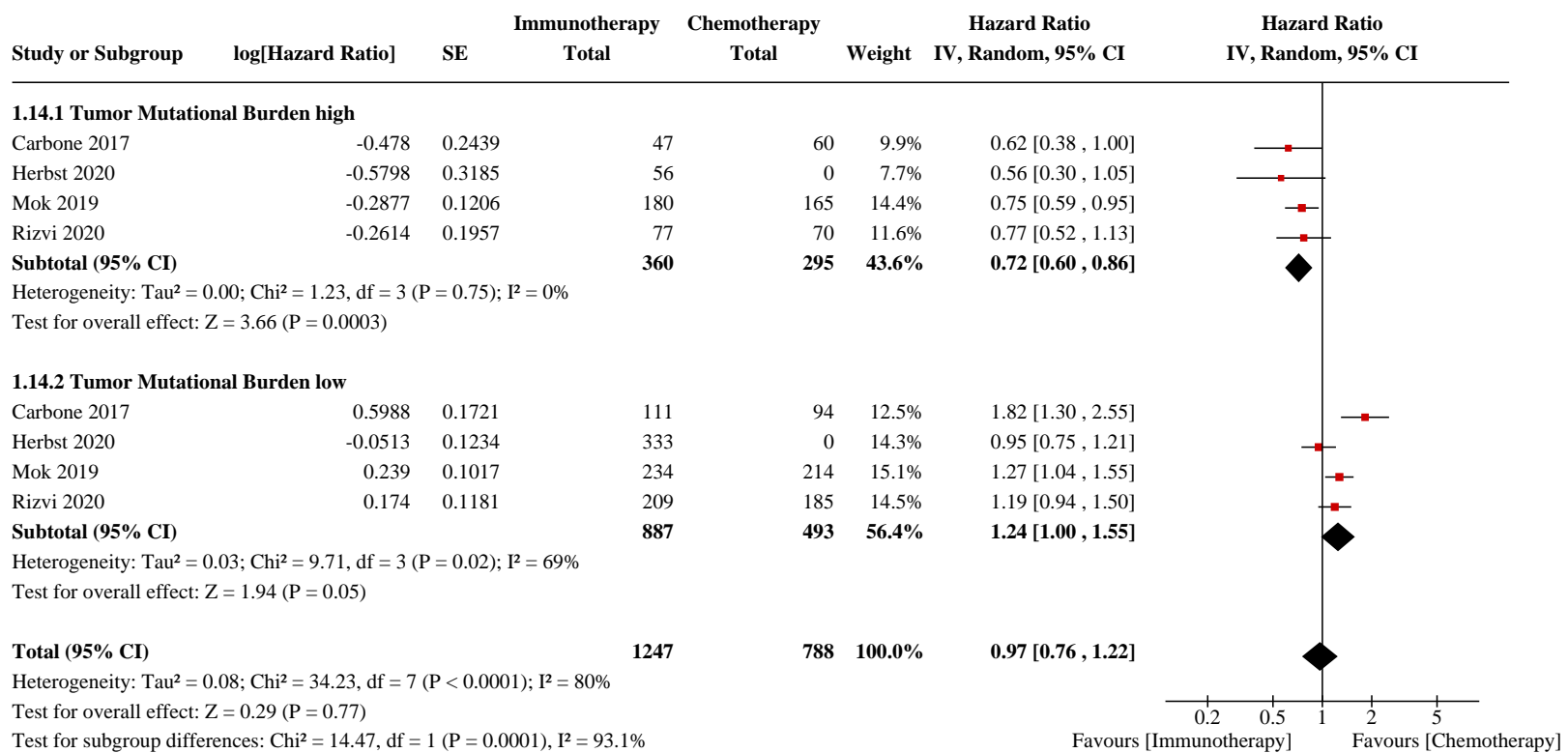


Analysis 1.15. Comparison 1: Single immunecheckpoint inhibitors vs chemotherapy, Outcome 15: Overall response rate by PDL1 expression

\begin{tabular}{|c|c|c|c|c|c|c|c|}
\hline & Immun & lerapy & Chem & erapy & & Risk Ratio & Risk Ratio \\
\hline Study or Subgroup & Events & Total & Events & Total & Weight & IV, Random, $95 \%$ CI & IV, Random, 95\% CI \\
\hline
\end{tabular}

\subsubsection{PDL1\#1\%}

\section{Herbst 2020}

Mok 2019

Subtotal $(95 \%$ CI $)$

Total events:

81
174

\section{7}

$88 \quad 277 \quad 34.1 \%$

$0.92[0.72,1.18]$

914

255

169

$637-65.9 \%$

$914100.0 \%$

$1.03[0.86,1.23]$

$0.99[0.86,1.15]$

Heterogeneity: $\mathrm{Tau}^{2}=0.00 ; \mathrm{Chi}^{2}=0.50, \mathrm{df}=1(\mathrm{P}=0.48) ; \mathrm{I}^{2}=0 \%$

Test for overall effect: $\mathrm{Z}=0.12(\mathrm{P}=0.90)$

\subsubsection{PDL1\#50\%}

Herbst 2020

Mok 2019

Reck 2016

Sezer 2020

Subtotal $(95 \%$ CI)

Total events:

$\begin{array}{rr}41 & 107 \\ 118 & 299 \\ 71 & 154 \\ 111 & 283 \\ & \mathbf{8 4 3}\end{array}$

07

28

$98 \quad 18.0 \%$

$1.34[0.90,1.99]$

$1.23[0.99,1.53]$

$1.22[0.94,1.59]$

$57 \quad 151 \quad 26.2 \%$

$1.93[1.47,2.53]$

Heterogeneity: $\mathrm{Tau}^{2}=0.03 ; \mathrm{Chi}^{2}=7.59, \mathrm{df}=3(\mathrm{P}=0.06) ; \mathrm{I}^{2}=60 \%$

Test for overall effect: $\mathrm{Z}=2.98(\mathrm{P}=0.003)$

Test for subgroup differences: $\mathrm{Chi}^{2}=6.50, \mathrm{df}=1(\mathrm{P}=0.01), \mathrm{I}^{2}=84.6 \%$

$1.40[1.12,1.75]$

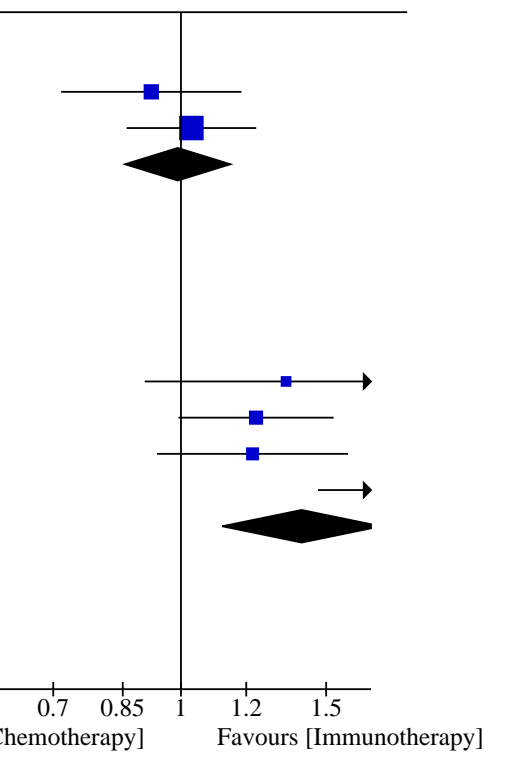

Analysis 1.16. Comparison 1: Single immunecheckpoint inhibitors vs chemotherapy, Outcome 16: Overall Response Rate by Tumor Mutational Burden

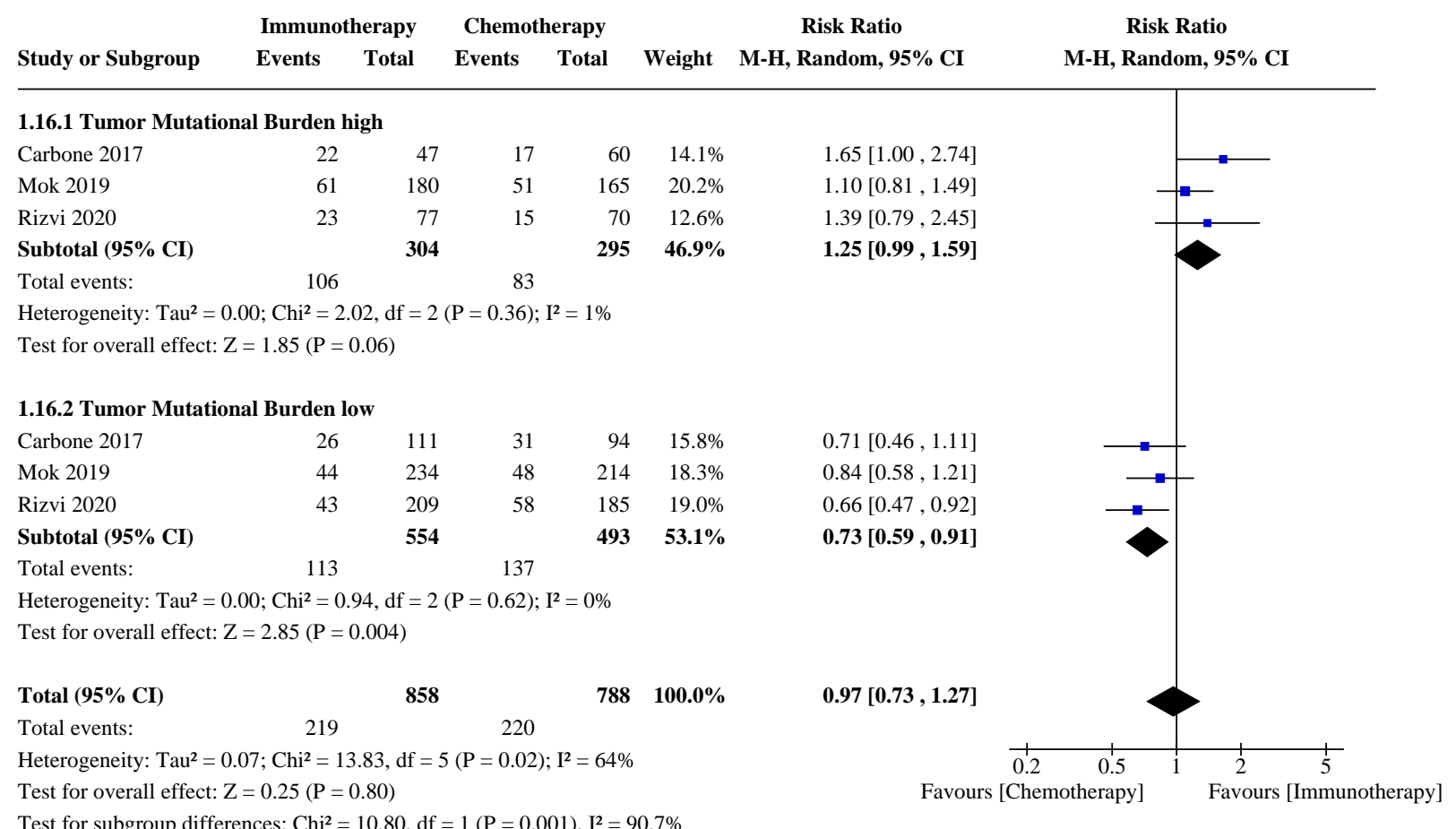

Single or combined immune checkpoint inhibitors compared to first-line platinum-based chemotherapy with or without bevacizumab for 


\section{Analysis 1.17. Comparison 1: Single immunecheckpoint inhibitors} vs chemotherapy, Outcome 17: Adverse Events grade 3-5

\begin{tabular}{|c|c|c|c|c|c|}
\hline & Immunotherapy & Chemotherapy & & Risk Ratio & Risk Ratio \\
\hline Study or Subgroup & Events & Events & Weight & M-H, Random, $95 \%$ CI & M-H, Random, $95 \%$ \\
\hline
\end{tabular}

1.17.1 Adverse Events grade 3-4

Carbone $2017 \quad 47$

Herbst 2020

$47 \quad 267$

$267 \quad 133$

\section{$263 \quad 17.9 \%$}

Mok 2019

Reck 2016

Rizvi 2020

Subtotal (95\% CI)

Total events:

Test for overall effect: $\mathrm{Z}=8.84(\mathrm{P}<0.00001)$

1.17.2 Adverse Events grade 5 (toxic deaths)

Carbone 2017

Herbst 2020

Mok 2019

Reck 2016

Rizvi 2020

Subtotal (95\% CI)

Total events:

$\begin{array}{rr}2 & 267 \\ 0 & 277 \\ 13 & 636 \\ 2 & 154 \\ 2 & 369 \\ & \mathbf{1 7 0 3}\end{array}$

\section{7}

636

154

369
703

19

$277 \quad 116$

$636 \quad 238$

154

369

1703

77

263

615

$615 \quad 22.5 \%$

$\begin{array}{ll}150 & 17.8 \% \\ 352 & 17.6 \%\end{array}$

$164391.4 \%$

680

Heterogeneity: $\mathrm{Tau}^{2}=0.00 ; \mathrm{Chi}^{2}=0.57, \mathrm{df}=4(\mathrm{P}=0.97) ; \mathrm{I}^{2}=0 \%$

Test for overall effect: $\mathrm{Z}=0.83(\mathrm{P}=0.40)$

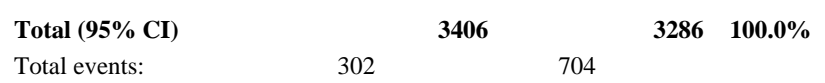

Heterogeneity: $\mathrm{Tau}^{2}=0.03 ; \mathrm{Chi}^{2}=15.27, \mathrm{df}=9(\mathrm{P}=0.08) ; \mathrm{I}^{2}=41 \%$

Test for overall effect: $\mathrm{Z}=8.93(\mathrm{P}<0.00001)$

Test for subgroup differences: $\mathrm{Chi}^{2}=4.08, \mathrm{df}=1(\mathrm{P}=0.04), \mathrm{I}^{2}=75.5 \%$

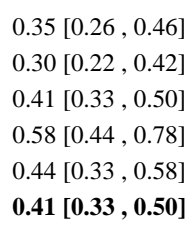

$0.35[0.26,0.46]$ $0.30[0.22,0.42]$ $0.41[0.33,0.50]$ $0.58[0.44,0.78]$ $0.44[0.33,0.58]$ $0.41[0.33,0.50]$

$0.66[0.11,3.90]$ $0.32[0.01,7.74]$ $0.90[0.43,1.89]$ $0.65[0.11,3.83]$ $0.64[0.11,3.78]$ $0.78[0.43,1.41]$

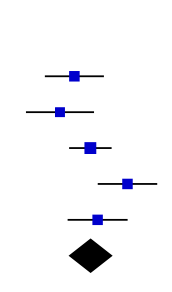

\begin{tabular}{|c|c|c|c|c|c|c|c|c|c|}
\hline \multirow[b]{2}{*}{ Study or Subgroup } & \multicolumn{3}{|c|}{ Checkpoints } & \multicolumn{3}{|c|}{ Chemotherapy } & \multirow{2}{*}{$\begin{array}{c}\text { Mean Difference } \\
\text { IV, Random, 95\% CI }\end{array}$} & \multirow{2}{*}{\multicolumn{2}{|c|}{$\begin{array}{c}\text { Mean Difference } \\
\text { IV, Random, 95\% CI }\end{array}$}} \\
\hline & Mean & SD & Total & Mean & SD & Total & & & \\
\hline Reck 2016 & 6.9 & 22.9328 & 150 & -0.9 & 24.1095 & 147 & $7.80[2.45,13.15]$ & & - \\
\hline
\end{tabular}

Analysis 1.19. Comparison 1: Single immunecheckpoint inhibitors vs chemotherapy, Outcome 19: Time to deterioration - QLQ-LC13

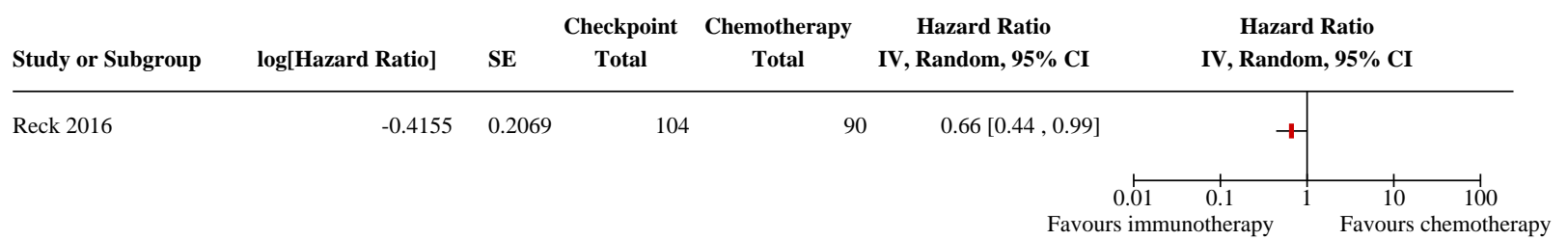

Single or combined immune checkpoint inhibitors compared to first-line platinum-based chemotherapy with or without bevacizumab for 
Analysis 1.20. Comparison 1: Single immunecheckpoint inhibitors vs chemotherapy, Outcome 20: HRQOL-QLQC30change from baseline at week 15-stable-PD-L1 $\geq 50 \%$

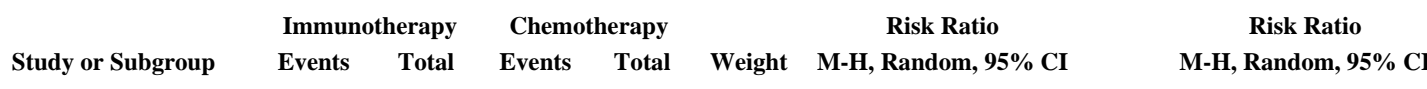

\subsubsection{GHS/QOL}

Reck 2016

Subtotal (95\% CI)

$58 \quad 150$

Total events:

58

150
150

64

$147 \quad 100.0 \%$

$147 \quad 100.0 \%$

64

Heterogeneity: Not applicable

Test for overall effect: $\mathrm{Z}=0.85(\mathrm{P}=0.39)$

1.20.2 Physical functioning

$\begin{array}{lcccccc}\text { Reck } 2016 & 80 & 150 & 58 & 147 & 100.0 \% & 1.35[1.05,1.74] \\ \text { Subtotal (95\% CI) } & & \mathbf{1 5 0} & & \mathbf{1 4 7} & \mathbf{1 0 0 . 0 \%} & \mathbf{1 . 3 5}[\mathbf{1 . 0 5}, \mathbf{1 . 7 4}] \\ \text { Total events: } & 80 & & 58 & & & \end{array}$

Heterogeneity: Not applicable

Test for overall effect: $\mathrm{Z}=2.36(\mathrm{P}=0.02)$

\subsubsection{Role functioning}

\section{Reck 2016}

Subtotal (95\% CI)

\section{3}

Total events:

63

Heterogeneity: Not applicable

Test for overall effect: $\mathrm{Z}=1.05(\mathrm{P}=0.30)$

\subsubsection{Emotional functioning}

Reck 2016

Subtotal (95\% CI)

$79 \quad 150$

Total events:

$1.11[0.88,1.39]$

$1.11[0.88,1.39]$
$0.89[0.68,1.17]$

$0.89[0.68,1.17]$

Heterogeneity: Not applicable

Test for overall effect: $\mathrm{Z}=0.87(\mathrm{P}=0.39)$

\subsubsection{Cognitive functioning}

$\begin{array}{lcccccc}\text { Reck } 2016 & 80 & 150 & 70 & 147 & 100.0 \% & 1.12[0.89,1.40] \\ \text { Subtotal }(\mathbf{9 5 \%} \text { CI) } & & \mathbf{1 5 0} & & \mathbf{1 4 7} & \mathbf{1 0 0 . 0 \%} & \mathbf{1 . 1 2}[\mathbf{0 . 8 9}, \mathbf{1 . 4 0}] \\ \text { Total events: } & 80 & & 70 & & & \end{array}$

Heterogeneity: Not applicable

Test for overall effect: $\mathrm{Z}=0.98(\mathrm{P}=0.33)$

1.20.6 Social functioning

$\begin{array}{lcccccc}\text { Reck } 2016 & 69 & 150 & 56 & 147 & 100.0 \% & 1.21[0.92,1.58] \\ \text { Subtotal }(\mathbf{9 5 \%} \text { CI) } & & \mathbf{1 5 0} & & \mathbf{1 4 7} & \mathbf{1 0 0 . 0 \%} & \mathbf{1 . 2 1}[\mathbf{0 . 9 2}, \mathbf{1 . 5 8}] \\ \text { Total events: } & 69 & & 56 & & & \end{array}$

Heterogeneity: Not applicable

Test for overall effect: $\mathrm{Z}=1.37(\mathrm{P}=0.17)$

1.20.7 Fatigue

Reck 2016

Subtotal (95\% CI)

Total events:

150

Heterogeneity: Not applicable

Test for overall effect: $\mathrm{Z}=0.92(\mathrm{P}=0.36)$

1.20.8 Nausea and vomiting

Reck 2016

Subtotal (95\% CI)

Total events:

109

150

Heterogeneity: Not applicable

Test for overall effect: $\mathrm{Z}=2.53(\mathrm{P}=0.01)$

1.20.9 Pain
-H, Random, 95\% CI 


\section{Analysis 1.20. (Continued)}

\subsubsection{Pain}

Reck 2016

Subtotal (95\% CI)

Total events:

Heterogeneity: Not applicable

Test for overall effect: $\mathrm{Z}=1.32(\mathrm{P}=0.19)$

\subsubsection{Dyspnoea}

\section{Reck 2016}

Subtotal (95\% CI)

Total events:

150

77

Heterogeneity: Not applicable

Test for overall effect: $\mathrm{Z}=1.92(\mathrm{P}=0.06)$

\subsubsection{Insomnia}

Reck 2016

Subtotal $(95 \%$ CI $)$

Total events:

$72 \quad 150$

Heterogeneity: Not applicable

Test for overall effect: $\mathrm{Z}=2.18(\mathrm{P}=0.03)$

\subsubsection{Appetite loss}

$\begin{array}{lrrrrrr}\text { Reck } 2016 & 72 & 150 & 61 & 147 & 100.0 \% & 1.16[0.90,1.49] \\ \text { Subtotal }(\mathbf{9 5 \%} \text { CI) } & & \mathbf{1 5 0} & & \mathbf{1 4 7} & \mathbf{1 0 0 . 0 \%} & \mathbf{1 . 1 6}[\mathbf{0 . 9 0}, \mathbf{1 . 4 9}] \\ \text { Total events: } & 72 & & 61 & & & \end{array}$

Heterogeneity: Not applicable

Test for overall effect: $\mathrm{Z}=1.12(\mathrm{P}=0.26)$

\subsubsection{Constipation}

\section{Reck 2016 \\ Subtotal $(95 \%$ CI $)$}

Total events:

teterogeneity: Not applicable

Test for overall effect: $\mathrm{Z}=0.85(\mathrm{P}=0.39)$

\subsubsection{Diarrhoea}

Reck 2016
Subtotal (95\% CI)

Total events:

117

150

117

Heterogeneity: Not applicable

Test for overall effect: $\mathrm{Z}=2.51(\mathrm{P}=0.01)$

\subsubsection{Financial difficulties}

Subtotal (95\% CI)

Total events:

Heterogeneity: Not applicable

Test for overall effect: $\mathrm{Z}=1.55(\mathrm{P}=0.12)$
$0.82[0.62,1.10]$

$0.82[0.62,1.10]$
$147 \quad 100.0 \%$

63

$\begin{array}{lll}59 & 147 & 100.0 \% \\ & \mathbf{1 4 7} & \mathbf{1 0 0 . 0} \%\end{array}$

$1.28[0.99,1.64]$

$1.28[0.99,1.64]$

$1.36[1.03,1.79]$

$1.36[1.03,1.79]$

$147 \quad 100.0 \%$

$147 \quad 100.0 \%$

$\begin{array}{rrrr}80 & 147 & 100.0 \% & 1.09[0.89,1.33] \\ & \mathbf{1 4 7} & \mathbf{1 0 0 . 0 \%} & \mathbf{1 . 0 9}[\mathbf{0 . 8 9}, \mathbf{1 . 3 3}]\end{array}$

80

95

$147 \quad 100.0 \%$

$147 \quad 100.0 \%$

$1.21[1.04,1.40]$

$1.21[1.04,1.40]$
95

$\begin{array}{rrr}80 & 147 & 100.0 \% \\ & \mathbf{1 4 7} & \mathbf{1 0 0 . 0} \%\end{array}$

$1.16[0.96,1.41]$
$1.16[0.96,1.41]$
80

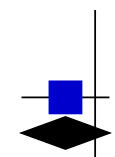

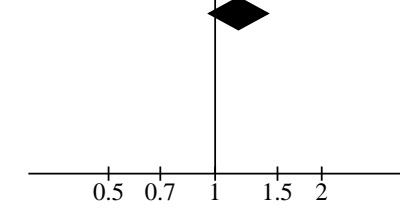

Favours [Chemotherapy] 
Analysis 1.21. Comparison 1: Single immunecheckpoint inhibitors vs chemotherapy, Outcome 21: HRQOL-QLQC30change from baseline at week 15 -deterioration - PDL1 $\geq 50 \%$

\begin{tabular}{|c|c|c|c|c|c|}
\hline & Immunotherapy & Chemotherapy & & Risk Ratio & Risk Ratio \\
\hline Study or Subgroup & Events & Events & Weight & M-H, Random, $95 \%$ CI & M-H, Random, $95 \%$ \\
\hline
\end{tabular}

\subsubsection{GHS/QOL}

Reck 2016

Subtotal (95\% CI)

Total events:

$32 \quad 150$

150

32

Heterogeneity: Not applicable

Test for overall effect: $\mathrm{Z}=1.68(\mathrm{P}=0.09)$

1.21.2 Physical functioning

$\begin{array}{lcc}\text { Reck } 2016 & 30 & 150 \\ \text { Subtotal }(\mathbf{9 5 \%} \text { CI) } & & \mathbf{1 5 0} \\ \begin{array}{l}\text { Total events: } \\ \text { Heterogeneity: Not applicable }\end{array} & 30 & \end{array}$

Heterogeneity: Not applicable

Test for overall effect: $\mathrm{Z}=2.99(\mathrm{P}=0.003)$

1.21.3 Role functioning

Subtotal (95\% CI)

$33 \quad 150$

33

Heterogeneity: Not applicable

Test for overall effect: $\mathrm{Z}=2.61(\mathrm{P}=0.009)$

1.21.4 Emotional functioning

Reck 2016

Subtotal $(95 \%$ CI)

Total events:

22

Heterogeneity: Not applicable

Test for overall effect: $\mathrm{Z}=0.61(\mathrm{P}=0.54)$

1.21.5 Cognitive functioning

Reck

Subtotal (95\% CI)

$40 \quad 150$

Total events:

40

Heterogeneity: Not applicable

Test for overall effect: $\mathrm{Z}=0.16(\mathrm{P}=0.87)$

1.21.6 Social functioning

Subtotal (95\% CI)

31

150

Total events:

31

Heterogeneity: Not applicable

Test for overall effect: $\mathrm{Z}=2.53(\mathrm{P}=0.01)$

\subsubsection{Fatigue}

Reck 2016

Subtotal (95\% CI)

Total events:

27

150

27

Heterogeneity: Not applicable

Test for overall effect: $\mathrm{Z}=4.27(\mathrm{P}<0.0001)$

1.21.8 Nausea and vomiting

$\begin{array}{lccccc}\text { Reck } 2016 & 17 & 150 & 35 & 147 & 100.0 \% \\ \text { Subtotal }(\mathbf{9 5 \%} \text { CI) } & & \mathbf{1 5 0} & & \mathbf{1 4 7} & \mathbf{1 0 0 . 0 \%} \\ \text { Total events: } & 17 & & 35 & & \end{array}$

Heterogeneity: Not applicable

Test for overall effect: $\mathrm{Z}=2.73(\mathrm{P}=0.006)$

1.21.9 Pain

50

$147 \quad 100.0 \%$
$147100.0 \%$

62
$0.71[0.48,1.06]$

$0.71[0.48,1.06]$

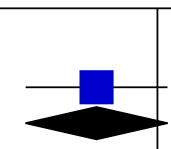

$0.55[0.38,0.82]$ $0.55[0.38,0.82]$

$0.61[0.42,0.88]$ $0.61[0.42,0.88]$

$1.20[0.67,2.14]$

$1.20[0.67,2.14]$

$1.03[0.70,1.51]$

$1.03[0.70,1.51]$
$0.61[0.41,0.89]$

$0.61[0.41,0.89]$
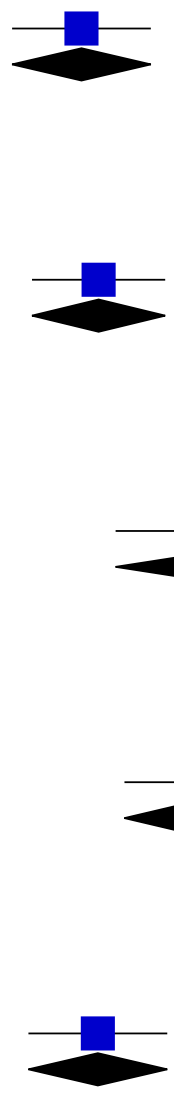

$0.43[0.29,0.63]$

$0.43[0.29,0.63]$

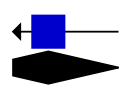

$0.48[0.28,0.81]$

$0.48[0.28,0.81]$ 


\section{Analysis 1.21. (Continued)}

\subsubsection{Pain}

Reck 2016

Subtotal (95\% CI)

Total events:

Heterogeneity: Not applicable

Test for overall effect: $\mathrm{Z}=1.24(\mathrm{P}=0.22)$

\subsubsection{Dyspnoea}

Reck 2016
Subtotal (95\% CI)

Total events:

20

(20

150

150

Heterogeneity: Not applicable

Test for overall effect: $\mathrm{Z}=3.53(\mathrm{P}=0.0004)$

\subsubsection{Insomnia}

\section{Reck 2016}

Subtotal (95\% CI)

Total events:

27

150

Heterogeneity: Not applicable

Test for overall effect: $\mathrm{Z}=1.75(\mathrm{P}=0.08)$

\subsubsection{Appetite loss}

Reck 2016

Total events:

150

Heterogeneity: Not applicable

Test for overall effect: $\mathrm{Z}=2.20(\mathrm{P}=0.03)$

\subsubsection{Constipation}

Reck 2016

27

Total events:

$27 \quad 150$

Heterogeneity: Not applicable

Test for overall effect: $\mathrm{Z}=2.12(\mathrm{P}=0.03)$

\subsubsection{Diarrhea}

Reck 2016

Subtotal (95\% CI)

21

$21 \quad 150$

Total events:

21

Heterogeneity: Not applicable

Test for overall effect: $\mathrm{Z}=1.16(\mathrm{P}=0.24)$

\subsubsection{Financial difficulties}

$\begin{array}{lccccc}\text { Reck } 2016 & 29 & 150 & 38 & 147 & 100.0 \% \\ \text { Subtotal (95\% CI) } & & \mathbf{1 5 0} & & \mathbf{1 4 7} & \mathbf{1 0 0 . 0 \%} \\ \text { Total events: } & 29 & & 38 & & \end{array}$

Heterogeneity: Not applicable

Test for overall effect: $\mathrm{Z}=1.34(\mathrm{P}=0.18)$

34

$147 \quad 100.0 \%$

$147 \quad 100.0 \%$

$147 \quad 100.0 \%$

$147100.0 \%$

$147 \quad 100.0 \%$

$147 \quad 100.0 \%$ $\begin{array}{ll}147 & 100.0 \% \\ \mathbf{1 4 7} & \mathbf{1 0 0 . 0 \%}\end{array}$

$0.75[0.47,1.18]$

$0.75[0.47,1.18]$

$0.43[0.27,0.68]$

$0.43[0.27,0.68]$

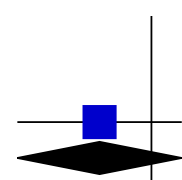

$0.68[0.44,1.05]$

$0.68[0.44,1.05]$

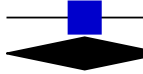

$0.59[0.37,0.94]$

$0.59[0.37,0.94]$

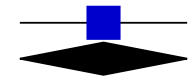

$0.63[0.41,0.97]$

$0.63[0.41,0.97]$

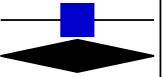

$0.73[0.44,1.23]$

$0.73[0.44,1.23]$

$0.75[0.49,1.15]$

$0.75[0.49,1.15]$
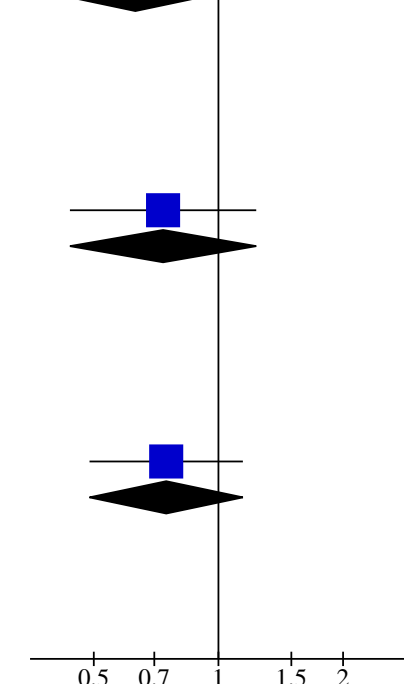

Favours [Immunotherapy] Favours [Chemotherapy] 
Analysis 1.22. Comparison 1: Single immunecheckpoint inhibitors vs chemotherapy, Outcome 22: HRQOL-QLQC30change from baseline at week 15 -improvement - PD-L1 $\geq 50 \%$

\begin{tabular}{|c|c|c|c|c|c|}
\hline & Immunotherapy & Chemotherapy & & Risk Ratio & Risk Ratio \\
\hline Study or Subgroup & Events & Events & Weigh & M-H, Random, 95\% CI & M-H, Random, $95 \%$ CI \\
\hline
\end{tabular}

\subsubsection{GHS/QOL}

Reck 2016

Subtotal $(95 \%$ CI)

Total events:

Heterogeneity: Not applicable

Test for overall effect: $\mathrm{Z}=2.42(\mathrm{P}=0.02)$

1.22.2 Physical functioning

$\begin{array}{lcccccc}\text { Reck } 2016 & 40 & 150 & 36 & 147 & 100.0 \% & 1.09[0.74,1.61] \\ \text { Subtotal }(\mathbf{9 5 \%} \text { CI) } & & \mathbf{1 5 0} & & \mathbf{1 4 7} & \mathbf{1 0 0 . 0 \%} & \mathbf{1 . 0 9}[\mathbf{0 . 7 4 , \mathbf { 1 . 6 1 }}] \\ \text { Total events: } & 40 & & 36 & & & \end{array}$

Heterogeneity: Not applicable

Test for overall effect: $\mathrm{Z}=0.43(\mathrm{P}=0.67)$

\subsubsection{Role functioning}

\section{Reck 2016}

Subtotal (95\% CI)

$54 \quad 150$

Total events:

54

$$
150
$$

Heterogeneity: Not applicable

Test for overall effect: $\mathrm{Z}=1.49(\mathrm{P}=0.14)$

\subsubsection{Emotional functioning}

$\begin{array}{lcccccc}\text { Reck } 2016 & 49 & 150 & 59 & 147 & 100.0 \% & 0.81[0.60,1.10] \\ \text { Subtotal }(\mathbf{9 5 \%} \text { CI) } & & \mathbf{1 5 0} & & \mathbf{1 4 7} & \mathbf{1 0 0 . 0 \%} & \mathbf{0 . 8 1}[\mathbf{0 . 6 0}, \mathbf{1 . 1 0}] \\ \text { Total events: } & 49 & & 59 & & & \end{array}$

Heterogeneity: Not applicable

Test for overall effect: $\mathrm{Z}=1.33(\mathrm{P}=0.18)$

\subsubsection{Cognitive functioning}

\begin{tabular}{|c|c|c|c|c|c|c|}
\hline Reck 2016 & 30 & 150 & 39 & 147 & $100.0 \%$ & $0.75[0.50,1.15]$ \\
\hline Subtotal $(95 \%$ CI $)$ & & 150 & & 147 & $100.0 \%$ & $0.75[0.50,1.15]$ \\
\hline Total events: & 30 & & 39 & & & \\
\hline \multicolumn{7}{|c|}{ Heterogeneity: Not applicable } \\
\hline \multicolumn{7}{|c|}{ Test for overall effect: $\mathrm{Z}=1.32(\mathrm{P}=0.19)$} \\
\hline \multicolumn{7}{|c|}{ 1.22.6 Social functioning } \\
\hline Reck 2016 & 50 & 150 & 41 & 147 & $100.0 \%$ & $1.20[0.85,1.69]$ \\
\hline Subtotal (95\% CI) & & 150 & & 147 & $100.0 \%$ & $1.20[0.85,1.69]$ \\
\hline Total events: & 50 & & 41 & & & \\
\hline \multicolumn{7}{|c|}{ Heterogeneity: Not applicable } \\
\hline \multicolumn{7}{|c|}{ Test for overall effect: $\mathrm{Z}=1.01(\mathrm{P}=0.31)$} \\
\hline \multicolumn{7}{|l|}{ 1.22.7 Fatigue } \\
\hline Reck 2016 & 77 & 150 & 47 & 147 & $100.0 \%$ & $1.61[1.21,2.13]$ \\
\hline Subtotal $(95 \%$ CI $)$ & & 150 & & 147 & $100.0 \%$ & $1.61[1.21,2.13]$ \\
\hline
\end{tabular}

$77-47$

Heterogeneity: Not applicable

Test for overall effect: $\mathrm{Z}=3.28(\mathrm{P}=0.001)$

1.22.8 Nausea and vomiting

$\begin{array}{lcccccc}\text { Reck } 2016 & 24 & 150 & 26 & 147 & 100.0 \% & 0.90[0.55,1.50] \\ \text { Subtotal }(\mathbf{9 5 \%} \text { CI) } & & \mathbf{1 5 0} & & \mathbf{1 4 7} & \mathbf{1 0 0 . 0 \%} & \mathbf{0 . 9 0}[\mathbf{0 . 5 5}, \mathbf{1 . 5 0}] \\ \text { Total events: } & 24 & & 26 & & & \end{array}$

Total events:

Heterogeneity: Not applicable

Test for overall effect: $\mathrm{Z}=0.39(\mathrm{P}=0.70)$

1.22.9 Pain
$1.51[1.08,2.10]$

$1.51[1.08,2.10]$

$1.29[0.92,1.81]$

$1.29[0.92,1.81]$

, $95 \%$ CI

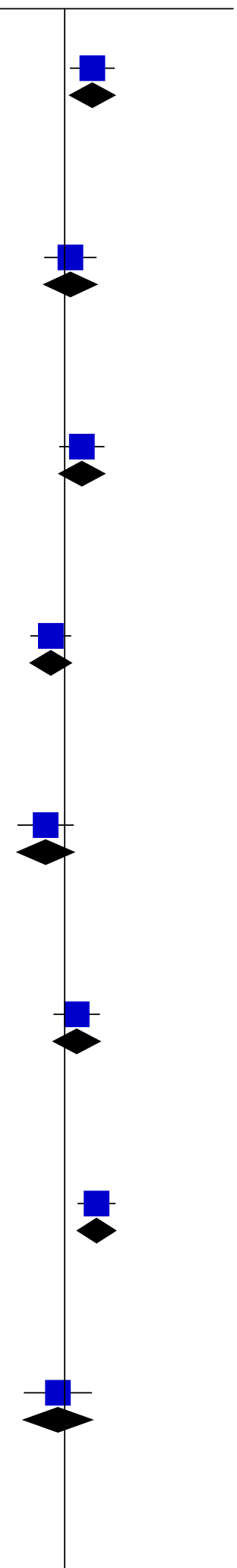

Single or combined immune checkpoint inhibitors compared to first-line platinum-based chemotherapy with or without bevacizumab for

Copyright (c) 2020 The Cochrane Collaboration. Published by John Wiley \& Sons, Ltd. 


\section{Analysis 1.22. (Continued)}

\subsubsection{Pain}

Reck 2016

Subtotal (95\% CI)

Total events:

Heterogeneity: Not applicable

Test for overall effect: $\mathrm{Z}=2.30(\mathrm{P}=0.02)$

\subsubsection{Dyspnoea}

\section{Reck 2016}

Subtotal (95\% CI)

$$
53
$$

150

Total events:

53

Heterogeneity: Not applicable

Test for overall effect: $\mathrm{Z}=1.24(\mathrm{P}=0.21)$

\subsubsection{Insomnia}

Reck 2016

Subtotal $(95 \%$ CI $)$

Total events:

51

150

150

Heterogeneity: Not applicable

Test for overall effect: $\mathrm{Z}=0.73(\mathrm{P}=0.46)$

\subsubsection{Appetite loss}

$\begin{array}{lrrrrrr}\text { Reck } 2016 & 55 & 150 & 48 & 147 & 100.0 \% & 1.12[0.82,1.54] \\ \text { Subtotal }(\mathbf{9 5 \%} \text { CI) } & & \mathbf{1 5 0} & & \mathbf{1 4 7} & \mathbf{1 0 0 . 0 \%} & \mathbf{1 . 1 2}[\mathbf{0 . 8 2}, \mathbf{1 . 5 4}] \\ \text { Total events: } & 55 & & 48 & & & \end{array}$

Heterogeneity: Not applicable

Test for overall effect: $\mathrm{Z}=0.73(\mathrm{P}=0.47)$

\subsubsection{Constipation}

$\begin{array}{lcccccc}\text { Reck } 2016 & 34 & 150 & 25 & 147 & 100.0 \% & 1.33[0.84,2.12] \\ \text { Subtotal }(\mathbf{9 5 \%} \text { CI) } & & \mathbf{1 5 0} & & \mathbf{1 4 7} & \mathbf{1 0 0 . 0 \%} & \mathbf{1 . 3 3}[\mathbf{0 . 8 4}, \mathbf{2 . 1 2}] \\ \text { Total events: } & 34 & & 25 & & & \end{array}$

Total events:

Test for overall effect: $\mathrm{Z}=1.21(\mathrm{P}=0.22)$

\subsubsection{Diarrhea}

Reck 2016
Subtotal (95\% CI)

Total events:

12

Heterogeneity: Not applicable

Test for overall effect: $\mathrm{Z}=2.14(\mathrm{P}=0.03)$

\subsubsection{Financial difficulties}

Subtotal (95\% CI)

Total events:

Heterogeneity: Not applicable

Test for overall effect: $\mathrm{Z}=0.53(\mathrm{P}=0.60)$
$1.39[1.05,1.84]$

$1.24[0.88,1.73]$

$1.24[0.88,1.73]$

$0.89[0.66,1.21]$
$1.39[1.05,1.84]$

$0.89[0.66,1.21]$

$147 \quad 100.0 \%$
$147 \quad 100.0 \%$

42

56
$0.49[0.25,0.94]$

$0.49[0.25,0.94]$

24

$147 \quad 100.0 \%$

$147 \quad 100.0 \%$

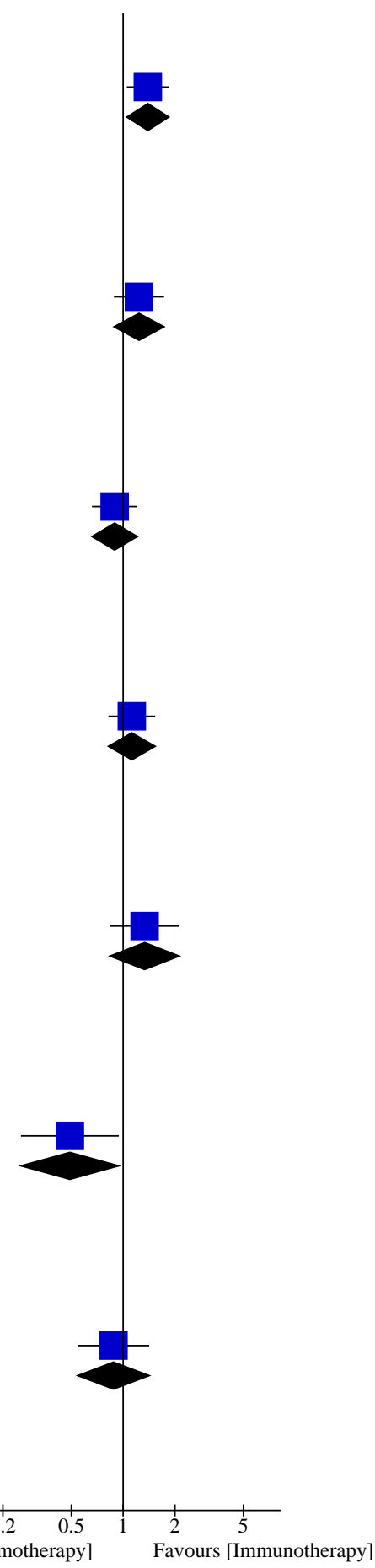

Single or combined immune checkpoint inhibitors compared to first-line platinum-based chemotherapy with or without bevacizumab for 
Comparison 2. Combined immune checkpoint inhibitors vs chemotherapy

\begin{tabular}{|c|c|c|c|c|}
\hline Outcome or subgroup title & $\begin{array}{l}\text { No. of } \\
\text { studies }\end{array}$ & $\begin{array}{l}\text { No. of } \\
\text { partici- } \\
\text { pants }\end{array}$ & Statistical method & Effect size \\
\hline $\begin{array}{l}2.1 \text { Overall Survival by PD-L1 ex- } \\
\text { pression }\end{array}$ & 2 & & Hazard Ratio (IV, Random, 95\% CI) & Subtotals only \\
\hline 2.1.1 PD-L1 <1\% & 2 & 532 & Hazard Ratio (IV, Random, 95\% CI) & $0.67[0.55,0.81]$ \\
\hline 2.1.2 PD-L1 $\geq 1 \%$ & 2 & 1378 & Hazard Ratio (IV, Random, 95\% CI) & $0.89[0.70,1.13]$ \\
\hline 2.1.3 PD-L1 $\geq 50 \%$ & 2 & 612 & Hazard Ratio (IV, Random, 95\% CI) & $0.72[0.59,0.89]$ \\
\hline $\begin{array}{l}2.2 \text { Overall Survival by Tumor Mu- } \\
\text { tational Burden }\end{array}$ & 2 & 1202 & Hazard Ratio (IV, Random, 95\% CI) & $0.75[0.54,1.05]$ \\
\hline $\begin{array}{l}\text { 2.2.1 Tumor Mutational Burden } \\
\text { low }\end{array}$ & 2 & 769 & Hazard Ratio (IV, Random, 95\% CI) & $0.93[0.61,1.43]$ \\
\hline $\begin{array}{l}\text { 2.2.2 Tumor Mutational Burden } \\
\text { high }\end{array}$ & 2 & 433 & Hazard Ratio (IV, Random, 95\% CI) & $0.60[0.44,0.82]$ \\
\hline $\begin{array}{l}\text { 2.3 Progression Free Survival by } \\
\text { Tumor Mutational Burden }\end{array}$ & 2 & 1202 & Hazard Ratio (IV, Random, 95\% CI) & $0.86[0.53,1.40]$ \\
\hline $\begin{array}{l}\text { 2.3.1 Tumor Mutational Burden } \\
\text { low }\end{array}$ & 2 & 769 & Hazard Ratio (IV, Random, 95\% CI) & $1.29[0.90,1.85]$ \\
\hline $\begin{array}{l}\text { 2.3.2 Tumor Mutational Burden } \\
\text { high }\end{array}$ & 2 & 433 & Hazard Ratio (IV, Random, 95\% CI) & $0.56[0.43,0.73]$ \\
\hline $\begin{array}{l}\text { 2.4 Overall Response Rate by Tu- } \\
\text { mor Mutational Burden }\end{array}$ & 2 & 822 & Risk Ratio (M-H, Random, 95\% Cl) & $1.25[0.53,2.96]$ \\
\hline $\begin{array}{l}\text { 2.4.1 Tumor Mutational Burden } \\
\text { low }\end{array}$ & 1 & 389 & Risk Ratio (M-H, Random, 95\% CI) & $0.53[0.37,0.77]$ \\
\hline $\begin{array}{l}\text { 2.4.2 Tumor Mutational Burden } \\
\text { high }\end{array}$ & 2 & 433 & Risk Ratio (M-H, Random, 95\% CI) & $1.83[1.40,2.39]$ \\
\hline 2.5 Adverse Events grade 3-5 & 2 & 3738 & Risk Ratio (M-H, Random, 95\% Cl) & $0.84[0.62,1.15]$ \\
\hline 2.5.1 Adverse events grade 3-4 & 2 & 1869 & Risk Ratio (M-H, Random, 95\% Cl) & $0.78[0.55,1.09]$ \\
\hline $\begin{array}{l}\text { 2.5.2 Adverse events grade } 5 \text { (toxic } \\
\text { deaths) }\end{array}$ & 2 & 1869 & Risk Ratio (M-H, Random, 95\% CI) & $1.51[0.65,3.48]$ \\
\hline
\end{tabular}


Analysis 2.1. Comparison 2: Combined immune checkpoint inhibitors vs chemotherapy, Outcome 1: Overall Survival by PD-L1 expression

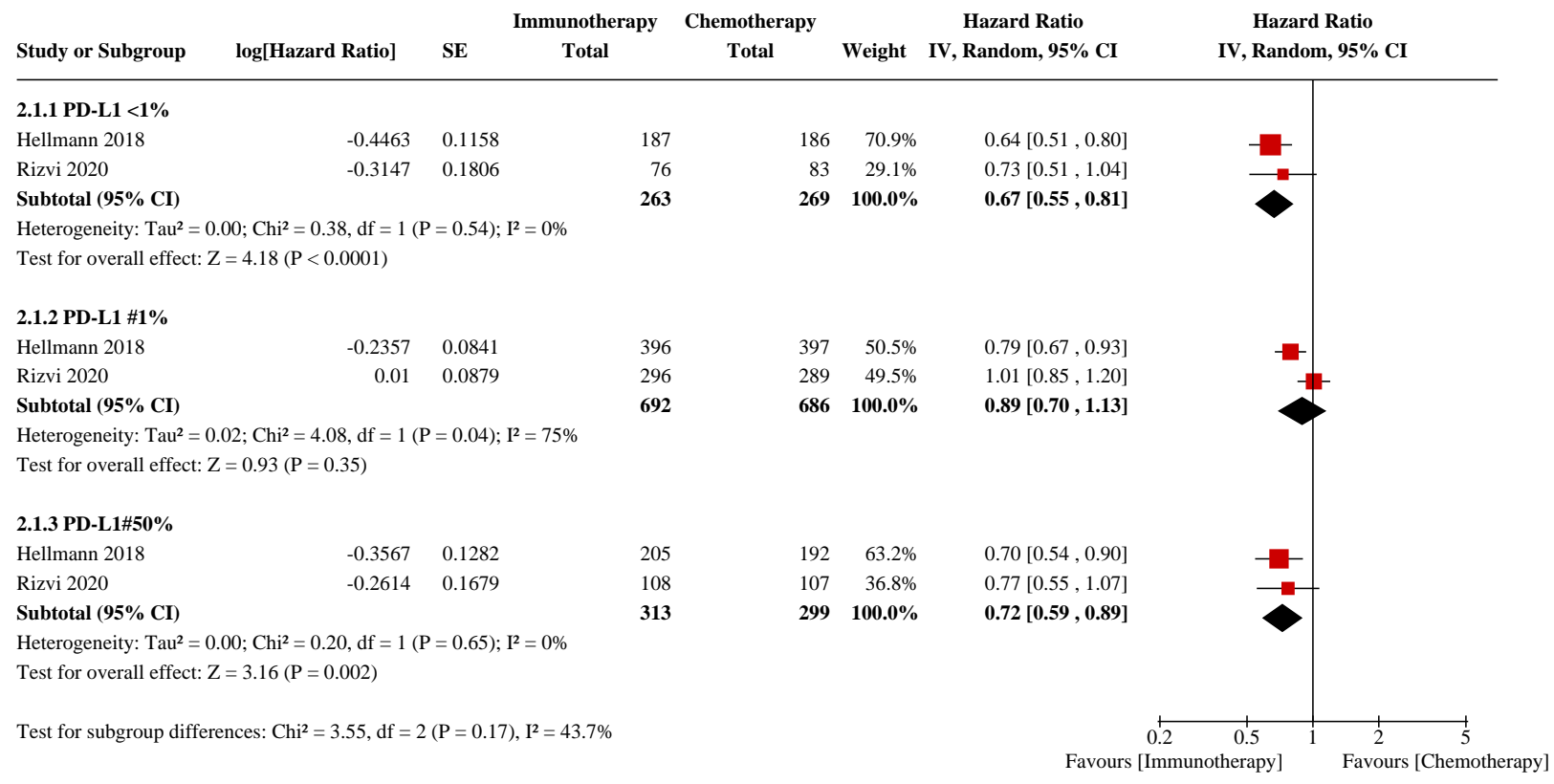

Analysis 2.2. Comparison 2: Combined immune checkpoint inhibitors vs chemotherapy, Outcome 2: Overall Survival by Tumor Mutational Burden

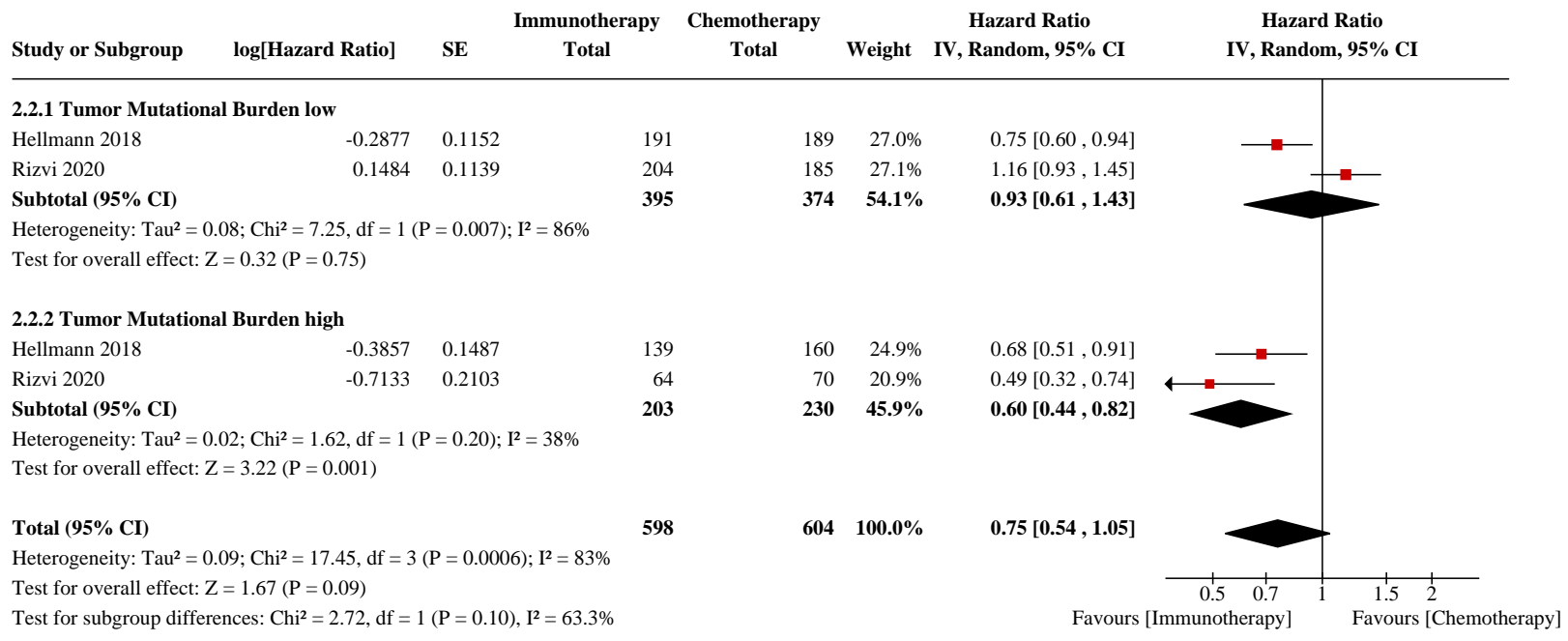


Analysis 2.3. Comparison 2: Combined immune checkpoint inhibitors vs chemotherapy, Outcome 3: Progression Free Survival by Tumor Mutational Burden

\begin{tabular}{|c|c|c|c|c|c|c|c|}
\hline Study or Subgroup & $\log [$ Hazard Ratio] & SE & $\begin{array}{c}\text { Immunotherapy } \\
\text { Total }\end{array}$ & $\begin{array}{c}\text { Chemotherapy } \\
\text { Total }\end{array}$ & Weight & $\begin{array}{c}\text { Hazard Ratio } \\
\text { IV, Random, 95\% CI }\end{array}$ & $\begin{array}{c}\text { Hazard Ratio } \\
\text { IV, Random, } 95 \% \text { CI }\end{array}$ \\
\hline \multicolumn{8}{|c|}{ 2.3.1 Tumor Mutational Burden low } \\
\hline Hellmann 2018 & 0.0677 & 0.1186 & 191 & 189 & $26.2 \%$ & $1.07[0.85,1.35]$ & - \\
\hline Rizvi 2020 & 0.4383 & 0.1145 & 204 & 185 & $26.3 \%$ & $1.55[1.24,1.94]$ & $\rightarrow$ \\
\hline Subtotal $(95 \%$ CI) & & & 395 & 374 & $52.5 \%$ & $1.29[0.90,1.85]$ & \\
\hline \multicolumn{8}{|c|}{ Heterogeneity: $\mathrm{Tau}^{2}=0.06 ; \mathrm{Chi}^{2}=5.05, \mathrm{df}=1(\mathrm{P}=0.02) ; \mathrm{I}^{2}=80 \%$} \\
\hline \multicolumn{8}{|c|}{ Test for overall effect: $Z=1.37(P=0.17)$} \\
\hline \multicolumn{8}{|c|}{ 2.3.2 Tumor Mutational Burden high } \\
\hline Hellmann 2018 & -0.5447 & 0.1704 & 139 & 160 & $24.6 \%$ & $0.58[0.42,0.81]$ & $\longrightarrow$ \\
\hline Rizvi 2020 & -0.6349 & 0.2164 & 64 & 70 & $22.9 \%$ & $0.53[0.35,0.81]$ & \\
\hline Subtotal $(95 \%$ CI) & & & 203 & 230 & $47.5 \%$ & $0.56[0.43,0.73]$ & \\
\hline \multicolumn{8}{|c|}{ Heterogeneity: $\mathrm{Tau}^{2}=0.00 ; \mathrm{Chi}^{2}=0.11, \mathrm{df}=1(\mathrm{P}=0.74) ; \mathrm{I}^{2}=0 \%$} \\
\hline \multicolumn{8}{|c|}{ Test for overall effect: $\mathrm{Z}=4.33(\mathrm{P}<0.0001)$} \\
\hline Total $(95 \%$ CI $)$ & & & 598 & 604 & $100.0 \%$ & $0.86[0.53,1.40]$ & \\
\hline \multicolumn{8}{|c|}{ Heterogeneity: $\mathrm{Tau}^{2}=0.22 ; \mathrm{Chi}^{2}=33.63, \mathrm{df}=3(\mathrm{P}<0.00001) ; \mathrm{I}^{2}=91 \%$} \\
\hline \multicolumn{7}{|c|}{ Test for overall effect: $\mathrm{Z}=0.60(\mathrm{P}=0.55)$} & 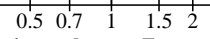 \\
\hline Test for subgroup diff & nces: $\mathrm{Chi}^{2}=13.29, \mathrm{df}=$ & $(\mathrm{P}=$ & $3), I^{2}=92.5 \%$ & & & F & otherapy] \\
\hline
\end{tabular}

Analysis 2.4. Comparison 2: Combined immune checkpoint inhibitors vs chemotherapy, Outcome 4: Overall Response Rate by Tumor Mutational Burden

\begin{tabular}{lccccccc} 
& \multicolumn{2}{c}{ Immunotherapy } & \multicolumn{2}{c}{ Chemotherapy } & \multicolumn{2}{c}{ Risk Ratio } & Risk Ratio \\
Study or Subgroup & Events & Total & Events & Total & Weight & M-H, Random, 95\% CI & M-H, Random, 95\% CI
\end{tabular}

2.4.1 Tumor Mutational Burden low

$\begin{array}{lcccccc}\text { Rizvi } 2020 & 34 & 204 & 58 & 185 & 33.7 \% & 0.53[0.37,0.77] \\ \text { Subtotal (95\% CI) } & & \mathbf{2 0 4} & & \mathbf{1 8 5} & \mathbf{3 3 . 7 \%} & \mathbf{0 . 5 3}[\mathbf{0 . 3 7}, \mathbf{0 . 7 7}] \\ \text { Total events: } & 34 & & 58 & & & \end{array}$

Heterogeneity: Not applicable

Test for overall effect: $\mathrm{Z}=3.31(\mathrm{P}=0.0009)$

2.4.2 Tumor Mutational Burden high

Hellmann 2018

Rizvi 2020

Subtotal (95\% CI)

$63 \quad 139$

Total events:

31

Heterogeneity: $\mathrm{Tau}^{2}=0.00 ; \mathrm{Chi}^{2}=0.91, \mathrm{df}=1(\mathrm{P}=0.34) ; \mathrm{I}^{2}=0 \%$

Test for overall effect: $\mathrm{Z}=4.40(\mathrm{P}<0.0001)$

Total $(95 \%$ CI)

407

Total events: 128 116

$415 \quad 100.0 \%$

Heterogeneity: $\mathrm{Tau}^{2}=0.53 ; \mathrm{Chi}^{2}=28.72, \mathrm{df}=2(\mathrm{P}<0.00001) ; \mathrm{I}^{2}=93 \%$

Test for overall effect: $\mathrm{Z}=0.52(\mathrm{P}=0.60)$

Test for subgroup differences: $\mathrm{Chi}^{2}=27.65, \mathrm{df}=1(\mathrm{P}<0.00001), \mathrm{I}^{2}=96.4 \%$

M-H, Random, $95 \%$ CI 
Analysis 2.5. Comparison 2: Combined immune checkpoint inhibitors vs chemotherapy, Outcome 5: Adverse Events grade 3-5

\begin{tabular}{|c|c|c|c|c|c|c|c|}
\hline \multirow[b]{2}{*}{ Study or Subgroup } & \multicolumn{2}{|c|}{ Immunotherapy } & \multicolumn{2}{|c|}{ Chemotherapy } & \multirow[b]{2}{*}{ Weight } & \multirow{2}{*}{$\begin{array}{c}\text { Risk Ratio } \\
\text { M-H, Random, } 95 \% \text { CI }\end{array}$} & \multirow{2}{*}{$\begin{array}{c}\text { Risk Ratio } \\
\text { M-H, Random, 95\% CI }\end{array}$} \\
\hline & Events & Total & Events & Total & & & \\
\hline \multicolumn{8}{|c|}{ 2.5.1 Adverse events grade 3-4 } \\
\hline Hellmann 2018 & 189 & 576 & 205 & 570 & $47.5 \%$ & $0.91[0.78,1.07]$ & \\
\hline Rizvi 2020 & 79 & 371 & 116 & 352 & $40.5 \%$ & $0.65[0.51,0.83]$ & \\
\hline Subtotal $(95 \%$ CI $)$ & & 947 & & 922 & $88.0 \%$ & $0.78[0.55,1.09]$ & \\
\hline Total events: & 268 & & 321 & & & & \\
\hline \multicolumn{8}{|c|}{ Heterogeneity: $\mathrm{Tau}^{2}=0.05 ; \mathrm{Chi}^{2}=5.33, \mathrm{df}=1(\mathrm{P}=0.02) ; \mathrm{I}^{2}=81 \%$} \\
\hline \multicolumn{8}{|c|}{ Test for overall effect: $\mathrm{Z}=1.46(\mathrm{P}=0.15)$} \\
\hline \multicolumn{8}{|c|}{ 2.5.2 Adverse events grade 5 (toxic deaths) } \\
\hline Hellmann 2018 & 8 & 576 & 6 & 570 & $7.4 \%$ & $1.32[0.46,3.78]$ & \\
\hline Rizvi 2020 & 6 & 371 & 3 & 352 & $4.6 \%$ & $1.90[0.48,7.53]$ & \\
\hline Subtotal (95\% CI) & & 947 & & 922 & $12.0 \%$ & $1.51[0.65,3.48]$ & \\
\hline Total events: & 14 & & 9 & & & & \\
\hline \multicolumn{8}{|c|}{ Heterogeneity: $\mathrm{Tau}^{2}=0.00 ; \mathrm{Chi}^{2}=0.17, \mathrm{df}=1(\mathrm{P}=0.68) ; \mathrm{I}^{2}=0 \%$} \\
\hline \multicolumn{8}{|c|}{ Test for overall effect: $\mathrm{Z}=0.96(\mathrm{P}=0.34)$} \\
\hline Total $(95 \%$ CI) & & 1894 & & 1844 & $100.0 \%$ & $0.84[0.62,1.15]$ & \\
\hline Total events: & 282 & & 330 & & & & \\
\hline \multirow{2}{*}{\multicolumn{5}{|c|}{$\begin{array}{l}\text { Heterogeneity: } \mathrm{Tau}^{2}=0.05 ; \mathrm{Chi}^{2}=7.44, \mathrm{df}=3(\mathrm{P}=0.06) ; \mathrm{I}^{2}=60 \% \\
\text { Test for overall effect: } \mathrm{Z}=1.08(\mathrm{P}=0.28)\end{array}$}} & & $\frac{+}{0.2}$ & 0.5 \\
\hline & & & & & & Favours [Immu & notherapy] \\
\hline
\end{tabular}

\section{APPENDICES}

\section{Appendix 1. CENTRAL search strategy}

\#1MeSH descriptor: [Carcinoma, Non-Small-Cell Lung] explode all trees

\#2nsclc

\#3lung cancer

\#4lung carcinom*

\#5lung neoplasm*

\#6lung tumor*

\#7lung tumour*

\#8non small cell*

\#9nonsmall cell ${ }^{\star}$

$\# 10$ (\#3 or \#4 or \#5 or \#6 or \#7) and (\#8 or \#9)

\#11first line

\#12naive

\#13\#11 or \#12

\#14MeSH descriptor: [Programmed Cell Death 1 Receptor] explode all trees \#15Programmed Cell Death 1

\#16PD-1 Receptor

\#17CD279 Antigen

\#18PD1 Receptor

\#19MeSH descriptor: [Programmed Cell Death 1 Ligand 2 Protein] explode all trees

\#20CD 273

\#21PD L2 Ligand

\#22B7 DC Ligand

\#23B7 DC Antigen*

\#24programmed death

\#25pd l1

$\# 26 \mathrm{pd} 12$

$\# 27 \mathrm{pd} 2$

\#28MeSH descriptor: [Immunotherapy] explode all trees 
\#291mmunotherap*

\#30durvalumab

\#31MEDI4736

\#32MEDI-4736

\#33Imfinzi

\#34avelumab

\#35MSB0010718C

\#36atezolizumab

\#37MPDL3280A

\#38Tecentriq

\#39RG7446

\#40RG 7446

\#41pembrolizumab

\#42lambrolizumab

\#43Keytruda

\#44MK3475

\#45MK 3475

\#46nivolumab

\#47MDX-1106

\#480NO-4538

\#49BMS-936558

\#500pdivo

\#51immune checkpoint inhibitor*

\#52MeSH descriptor: [Ipilimumab] explode all trees

\#53Ipilimumab

\#54Yervoy

\#55MDX010

\#56MDX 010

\#57tremelimumab

\#58ticilimumab

\#59CP $675^{\star}$

\#60CP675*

\#61MeSH descriptor: [Drug Therapy] explode all trees

\#62Drug Therap*

\#63Chemotherap*

\#64Pharmacotherap*

\#65MeSH descriptor: [Antineoplastic Agents] explode all trees

\#66Antineoplas ${ }^{\star}$

\#67Antitumo*

\#68Anticancer

$\# 69 \# 14$ or \#15 or \#16 or \#17 or \#18 or \#19 or \#20 or \#21 or \#22 or \#23 or \#24 or \#25 or \#26 or \#27 or \#28 or \#29 or \#30 or \#31 or \#32 or \#33 or \#34 or \#35 or \#36 or \#37 or \#38 or \#39 or \#40 or \#41 or \#42 or \#43 or \#44 or \#45 or \#46 or \#47 or \#48 or \#49 or \#50 or \#51 or \#52 or \#53 or $\# 54$ or \#55 or \#56 or \#57 or \#58 or \#59 or \#60 or \#61 or \#62 or \#63 or \#64 or \#65 or \#66 or \#67 or \#68

$\# 70 \# 10$ and \#13 and \#69

\section{Appendix 2. MEDLINE search strategy}

\#1,"Search Carcinoma, Non-Small-Cell Lung[MeSH Terms]"

\#2,"Search nsclc"

\#3,"Search lung cancer*"

\#4,"Search lung carcinom*"

\#5,"Search lung neoplasm*"

\#6,"Search lung tumor*"

\#,"Search lung tumour*"

\#8,"Search non small cell*"

\#9,"Search nonsmall cell*"

\#10,"Search (\#3 OR \#4 OR \#5 OR \#6 OR \#7) AND (\#8 OR \#9)"

\#11,"Search \#1 OR \#2 OR \#10"

$\# 16, "$ Search (first line) OR naive"

\#19,"Search ((((programmed cell death 1 receptor[MeSH Terms]) OR Programmed Cell Death 1) OR PD-1 Receptor) OR CD279 Antigen*) OR PD1 Receptor" 
\#22,"Search ((((programmed cell death 1 ligand 2 protein[MeSH Terms]) OR CD273 Antigen) OR PD L2 Ligand) OR B7 DC Ligand) OR B7 DC Antigen*"

\#29,"Search (((programmed death) OR pd l1) OR pd I2) OR pd 2"

\#31,"Search (immunotherapy[MeSH Terms]) OR Immunotherap*"

\#34,"Search (((((((((durvalumab) OR MEDI4736) OR MEDI-4736) OR Imfinzi) OR avelumab) OR MSB0010718C) OR atezolizumab) OR MPDL3280A) OR Tecentriq) OR RG7446)

\#36,"Search ((((pembrolizumab) OR lambrolizumab) OR Keytruda) OR MK3475) OR MK 3475"

\#38,"Search ((((nivolumab) OR MDX-1106) OR ONO-4538) OR BMS-936558) OR Opdivo"

\#41,"Search immune checkpoint inhibitor*"

\#43,"Search ((((Ipilimumab[MeSH Terms]) OR Yervoy) OR MDX 010) OR MDX010) OR MDX CTLA 4"

\#45,"Search tremelimumab OR ticilimumab OR CP $675^{*}$ OR CP675*"

\#48,"Search (((Drug Therapy[MeSH Terms]) OR Drug Therap*) OR Chemotherap*) OR Pharmacotherap*"

\#51,"Search (((Antineoplastic Agents[MeSH Terms]) OR Antineoplastic $\left.{ }^{\star}\right)$ OR Antitumo*) OR Anticancer"

\#52,"Search \#19 OR \#22 OR \#29 OR \#31 OR \#34 OR \#36 OR \#38 OR \#41 OR \#43 OR \#45 OR \#48 OR \#51"

\#53,"Search \#11 AND \#16 AND \#52"

\#54,"Search ((((((randomized controlled trial[Publication Type]) OR randomized[Title/Abstract]) OR placebo[Title/Abstract]) OR drug therapy[MeSH Subheading]) OR randomly[Title/Abstract]) OR trial[Title/Abstract]) OR groups[Title/Abstract]"

\#55,"Search (animals[MeSH Terms]) NOT humans[MeSH Terms]"

\#56,"Search \#54 NOT \#55"

\#57,"Search \#53 AND \#56"

\section{Appendix 3. Embase search strategy}

\#5 \#1 AND \#2 AND \#3 AND \#4

\#4 'crossover procedure'/exp OR 'double-blind procedure'/exp OR 'randomized controlled trial'/exp OR 'single-blind procedure'/exp OR random ${ }^{\star}$ OR factorial* ${ }^{*}$ R crossover ${ }^{\star}$ OR (cross NEXT/1 over ${ }^{\star}$ ) OR placebo* OR (doubl* NEAR/1 blind ${ }^{\star}$ ) OR (singl* NEAR/1 blind ${ }^{\star}$ ) OR assign* OR allocat* OR volunteer*

'programmed death 1 receptor'/exp OR 'programmed death 1 ligand 2'/exp OR 'programmed death 1 ligand 1'/exp OR 'anti programmed death':ab,ti OR 'programmed death ligand':ab,ti OR 'programmed death 2':ab,ti OR 'programmed death $1^{\prime}$ :ab,ti OR 'target ${ }^{\star}$ pd-l1':ab,ti OR 'target* pdI2':ab,ti OR 'target* pd-1':ab,ti OR 'pd-l1 block ${ }^{\star}:$ ab,ti OR 'pd-l2 block':ti,ab OR 'pd-1 block':ab,ti OR 'pd-l1 inhibitor*':ab,ti OR 'pd-l2 inhibitor*':ab,ti OR 'pd-1 inhibitor*':ab,ti OR 'anti pd-l1':ab,ti OR 'anti pd-l2':ab,ti OR 'anti pd-1':ab,ti OR 'immunotherap*':ab,ti OR 'immunotherapy'/exp OR 'durvalumab':ab,ti OR 'avelumab':ab,ti OR 'atezolizumab':ab,ti OR 'pembrolizumab':ab,ti OR 'nivolumab':ab,ti OR 'immune checkpoint inhibitor*':ab,ti OR 'ono-4538':ti,ab OR 'bms-936558':ti,ab OR 'ipilimumab':ti,ab OR 'tremelimumab':ti,ab OR 'ticilimumab':ti,ab OR 'pembrolizumab':ti,ab OR 'lambrolizumab':ti,ab OR 'cytotoxic t lymphocyte antigen 4'/exp OR ctla4:ti,ab OR keytruda:ti,ab OR mdx1106:ti,ab OR opdivo:ti,ab OR 'mk 3475':ti,ab OR mpdl3280a:ti,ab OR yervoy:ti,ab OR 'mdx 010 ':ti,ab OR 'mdx 101':ti,ab OR 'cp 675,206':ti,ab OR 'chemotherap*':ab,ti OR 'chemotherapy'/exp OR 'antineoplastic agent'/exp

\#2 'first line':ti,ab OR 'first-line':ti,ab OR naive:ti,ab

\#1

'lung tumor'/exp OR 'non small cell lung cancer'/exp OR ((lung NEXT/1 carcinom*):ab,ti) OR ((lung NEXT/1 neoplasm* ${ }^{\star}$ :ab,ti) OR 'lung cancer':ab,ti OR nsclc:ab,ti OR 'non small cell lung':ab,ti OR ((lung NEXT/1 tumour ${ }^{\star}$ :ab,ti) OR 'nonsmall cell lung':ab,ti OR 'non-small cell lung':ab,ti OR 'lung carcinoma'/exp OR 'lung cancer'/exp

\section{Appendix 4. clinicaltrials.gov search strategy}

$\# 1$ non small cell lung cancer

\#2nsclc

\#3immunotherapy

\#4PD1

\#5PDL1

Single or combined immune checkpoint inhibitors compared to first-line platinum-based chemotherapy with or without bevacizumab for people with advanced non-small cell lung cancer (Review)

Copyright $\odot 2020$ The Cochrane Collaboration. Published by John Wiley \& Sons, Ltd. 
\#6CTLA

date of last search 22.01.2020

\section{HISTORY}

Protocol first published: Issue 2, 2019

Review first published: Issue 12, 2020

\section{CONTRIBUTIONS OF AUTHORS}

Writing the protocol: RF, RM, MI, SPB, CM, VW.

Designing search strategies and monthly searches: FC

Selection of studies: RF, CM, RM, MI

Assessement of risk of bias: RF, RM

Extraction of data: MI, RF, SPB, RM

Conduct of the analysis: RF, SPB, MI, RM

Writing of the final review: RF, RM, MI, SPB, CM, VW

Critical appraisal of the final review paper: VW

\section{DECLARATIONS OF INTEREST}

Roberto Ferrara: none known.

Martina Imbimbo: received payment for development of educational presentations from BMS, case report pseudoprogression from immunotherapy and is Principal Investigator for phase I studies sponsored by Medimmune and BMS.

Sophie Paget-Bailly: declares a payment from BMS for the development of an educational presentation and the travel and accomodations to attend the same presentation.

Reem Malouf: none known.

François Calais: none known.

Corynne Marchal: none known.

Virginie Westeel received payment for board membership from MSD, BMS, Takeda, Roche, Astra Zeneca, for lectures including service on speakers bureaus from BMS, Astra Zeneca, Roche, MSD, for Payment for development of educational presentations from BMS, Astra Zeneca, Roche, for travel accommodations and meeting expenses from BMS, Astra Zeneca, Roche, Pfizer, Boehringer Ingelheim.Virginie Westeel declares payment to institution for the conduct of clinical trials from Abbvie, Boehringer Ingelheim, Bristol Myers Squibb, Fresenius, Lilly, MSD, Merck Serono, Novartis, Roche.

\section{SOURCES OF SUPPORT}

\section{Internal sources}

- No sources of support supplied

\section{External sources}

- INCa, France

French National Cancer Institute (INCa n²017-186)

\section{DIFFERENCES BETWEEN PROTOCOL AND REVIEW}

\section{Background}

In the background section we modified the description of the interventions according to the characteristics of the included studies.

\section{Methods}

Single or combined immune checkpoint inhibitors compared to first-line platinum-based chemotherapy with or without bevacizumab for people with advanced non-small cell lung cancer (Review)

Copyright (c) 2020 The Cochrane Collaboration. Published by John Wiley \& Sons, Ltd. 
We removed doublet immune checkpoint inhibitors versus single-agent immune checkpoint inhibitor in the first-line setting as it was inconsistent with the tile of our review.

Although we initially developed a data extraction form on Covidence as indicated in the protocol, in the final review, we used an Excel sheet to extract data from included studies because it was easier and more intuitive than Covidence.

Although we specified in the methods to report PFS and OS survival rates at specific time points, we did not perform this analysis because survival rates were not available from the majority of included studies. If these data are available in the future we will report them as RRs.

In the "type of studies" of the methods section, we deleted the sentence considering studies with cross-over designs. Indeed it was not relevant in the designs used in clinical trials in oncology setting.

\section{'Summary of findings' tables}

We initially planned to include the following OS, PFS, ORR, AEs grades 3 to 4 and 5, and HRQoL outcomes. The included trials in this review reported outcomes by different PDL-1 levels. therefore we found it necessary to select PD-L1 $\geq 50 \%$ as it was the most common used level. The AEs were not reported by PD-L1 expression.

We provided two 'Summary of findings' tables instead of one:

1. Single immune checkpoint inhibitors compared to chemotherapy for people with advanced non-small cell lung cancer (Summary of findings 1 )

2. Combined immune checkpoint inhibitors compared to chemotherapy for people with advanced non-small cell lung cancer (Summary of findings 2)

\section{Subgroups}

Due to lack of data we were unable to carry out the analysis for the subsequent subgroups that were inially described in the protocol.

- Immune checkpoint inhibitor or immuno-oncology therapy class (anti-PD-1, anti-PD-L1, anti-CTLA4)

- Inclusion or exclusion of participants with brain metastasis

- Follow-up duration

- liver metastases

- Presence of molecular alterations (EGFR mutation, ALK and ROS1 rearrangements)

The analysis of other subgroups reported in the protocol such as age, gender, performance status, smoking status, NSCLC histology and TMB expression (high versus low) were carried out.

In the protocol $\geq 50 \%, \geq 20 \%$, or $\geq 1 \%$ PD-L1 expression thresholds were initially used, but in the review we chose to regroup PD-L1 categories in "positive" (PD-L1 TPS $\geq 1 \%$ or TC1-2-3/IC1-2-3), "negative" (PD-L1 TPS <1\% or TC0/IC0) or "high" (PD-L1 TPS $\geq 50 \%$ or PD-L1 TC3/IC3).

\section{INDEX TERMS}

\section{Medical Subject Headings (MeSH)}

Antibodies, Monoclonal [therapeutic use]; Antibodies, Monoclonal, Humanized [therapeutic use]; Antineoplastic Combined Chemotherapy Protocols [adverse effects] [^therapeutic use]; B7-H1 Antigen [metabolism]; Bevacizumab [adverse effects] [*therapeutic use]; Bias; Carcinoma, Non-Small-Cell Lung [*drug therapy] [mortality] [pathology]; Immune Checkpoint Inhibitors [adverse effects] [ ${ }^{*}$ therapeutic use]; Lung Neoplasms [ ${ }^{\star}$ drug therapy] [mortality] [pathology]; Nivolumab [adverse effects] [therapeutic use]; Platinum Compounds [adverse effects] [therapeutic use]; Progression-Free Survival; Randomized Controlled Trials as Topic

\section{MeSH check words}

Aged; Female; Humans; Male; Middle Aged 